$$
\begin{gathered}
\text { ORNL-3455 } \\
\text { UC-34-Physics } \\
\text { TID-4500 (20th ed., Rev.) }
\end{gathered}
$$

\title{
A LITERATURE SURVEY OF NONELASTIC \\ REACTIONS FOR NUCLEONS AND PIONS \\ INCIDENT ON COMPLEX NUCLEI AT \\ ENERGIES BETWEEN $20 \mathrm{MeV}$ AND $33 \mathrm{GeV}$ \\ H. W. Bertini
}

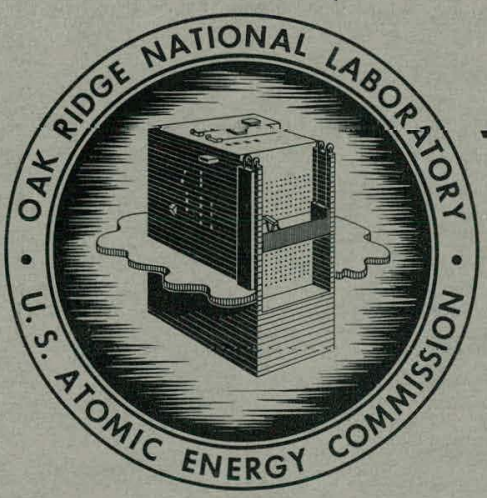

\section{OAK RIDGE NATIONAL LABORATORY}

$$
\text { operated by }
$$

UNION CARBIDE CORPORATION

$$
\text { for the }
$$

U.S. ATOMIC ENERGY COMMISSION 


\section{DISCLAIMER}

This report was prepared as an account of work sponsored by an agency of the United States Government. Neither the United States Government nor any agency Thereof, nor any of their employees, makes any warranty, express or implied, or assumes any legal liability or responsibility for the accuracy, completeness, or usefulness of any information, apparatus, product, or process disclosed, or represents that its use would not infringe privately owned rights. Reference herein to any specific commercial product, process, or service by trade name, trademark, manufacturer, or otherwise does not necessarily constitute or imply its endorsement, recommendation, or favoring by the United States Government or any agency thereof. The views and opinions of authors expressed herein do not necessarily state or reflect those of the United States Government or any agency thereof. 


\section{DISCLAIMER}

Portions of this document may be illegible in electronic image products. Images are produced from the best available original document. 
Printed in USA. Price: $\$ 2.50$ Available from the

Office of Technical Services

U. S. Department of Commerce

Washington 25, D. C.

\section{LEGAL NOTICE}

This report was prepared as an account of Government sponsored work. Neither the United States, nor the Commission, nor any person acting on behalf of the Commission:

A. Makes any warranty or representation, expressed or implied, with respect to the accuracy, completeness, or usefulness of the information contained in this report, or that the use of any information, apparatus, method, or process disclosed in this report may not infringe privately owned rights; or

B. Assumes any liabilities with respect to the use of, or for damages resulting from the use of any information, apparatus, method, or process disclosed in this report.

As used in the above, "person acting on behalf of the Commission" includes any employee or contractor of the Commission, or employee of such contractor, to the extent that such employee or contractor of the Commission, or employee of such contractor prepares, disseminates, or provides access to, any information pursuant to his employment or contract with the Commission, or his employment with such contractor. 
ORNL-3455

Contract No. W-7405-eng-26

Neutron Physics Division

A LITERATURE SURVEY OF NONELASTIC REACTIONS FOR NUCLEONS AND PIONS INCIDEINT ON COMPIEX NUCLEI AT EINERGIES BETWEENI $20 \mathrm{MeV}$ AND $33 \mathrm{GeV}$

H. W. Bertini

Date Issued

AUG 231963

OAK RIDGE NATIONAL LABORATORY

Oak Ridge, Tennessee

operated by

UNION CARBIDE CORPORATION

for the

U.S. ATOMIC ENERGY COMMISSION 


\section{THIS PAGE}

\section{WAS INTENTIONALLY}

LEFT BLANK 


\begin{abstract}
A literature survey has been made of experimental data on nonelastic reactions of protons, neutrons, $\pi^{+}$, and $\pi^{-}$with complex nuclei for incident energies of $20 \mathrm{MeV}$ to $33 \mathrm{GeV}$. Only those reactions in which nucleons and $\pi$-mesons are emitted were considered. The survey was in general confined to publications for which abstracts appear in the journal Physics Abstracts. For pions the period covered is January 1955 to November 1962 and for nucleons it is for January 1957 to November 1962.
\end{abstract}


Page No.

Abstract

General

Notation

Total Reaction Cross Sections as a Function of Incident Proton Energy

Differential Cross Sections as a Function of the Incident Proton Energy and the Other Variables Indicated

Tabulation of Reactions for Various Incident 
GENERAL

This report contains the result of a literature search for experimental data on the nonelastic reactions of protons, neutrons, $\pi^{+}$, and $\pi^{-}$ at energies between $20 \mathrm{MeV}$ and $33 \mathrm{GeV}$ incident on complex nuclei. The search was restricted to those reactions which lead to the emission of nucleons and $\pi$ mesons.

The journal Physics Abstracts (Section A of Science Abstracts) was used as a reference for most of the articles listed, with issues from January 1955 through November 1962 reviewed for the incident-pion data and issues from January 1957 through November 1962 reviewed for the incidentnucleon data.

The data are presented in tabular form, with the material grouped according to the incident particles protons, neutrons, $\pi^{+}$, and $\pi^{-}$in that order. The target nuclei for each type of incident particle are arranged according to increasing mass numbers and for each mass number the particle energies are listed in the order of increasing energy. When the energies - at which the experiment was conducted were too numerous to be listed individually, only the range of values is given, with the numerical sequence being determined by the mean energy of the range. Space limitations made it necessary to list only representative quantities for articles that included a great many quantities measured. The experimental error listed in the tabulation represents either the cited author's estimate or, when the amount of data included in the article was large, represents the error limits between which the bulk of the data lie. When the data were presented in graphical form in the article, the errors listed were estimated (by the literature searcher) from the error bars. In no case was an effort made to distinguish between statistical and systematic errors for the values given in the tabulation. The detector, the installation, and the accelerator are given for general information when they were cited in the articles.

A bibliography is presented following the main listing. The numbers in parentheses listed under each entry represent the numerical order in which the reference appeare in the tabulation. 
NOTATION

The notation used is intended to correspond in general with that suggested by the National Cross Section Advisory Group. ${ }^{1}$ The incident and emitted particles are indicated by appropriate subscripts to the letter $\sigma$ separated by a comma. A capital letter follows the letter designating the incident particle to indicate reactions of a general class. A symbol $E$ for the incident particle energy is included in the definitions to make them more general. As a rule the symbol for the incident particle energy is unprimed and has no subscript, while that for the emitted particles has either a prime or a subscript. In the present survey the symbol for the incident particle energy is not used, because the incident energy is listed separately.

In the examples which follow it is assumed that the incident particle is a proton, but the same notation with different subscripts applies to other incident particles.

1. John R. Stehn, Compilation of Requests for Nuclear Cross Section Measurements, WASH-1040 EANDC (Sept. 1962). 
Total Reaction Cross Sections as a Function of Incident Proton Energy

$$
\begin{aligned}
& \sigma_{\mathrm{pT}}(E) \equiv \text { Total cross section. } \\
& \sigma_{p, p}(E) \equiv \text { Elastic cross section. } \\
& \sigma_{p X}(E) \equiv \text { Cross section for all nonelastic processes. } \\
& \sigma_{p, p^{\prime}}(E) \equiv \text { Cross section for the inelastic scattering of the } \\
& \dot{\sigma}_{p D}(E) \equiv \text { Cross section for the disappearance of the proton.* } \\
& \sigma_{p, x p y}(E) \equiv \text { Cross section for proton production, i.e., the cross sec- } \\
& \sigma_{p, x p}(E) \equiv \text { Cross section for proton production only (i.e., any } \\
& \text { number of protons but no other type of particle). } \\
& \sigma_{p, l p}(E) \equiv \text { Cross section for producing one proton only by nonelastic } \\
& \text { processes (includes } \sigma_{p, p^{\prime}} \text { ). } \\
& \begin{aligned}
\sigma_{p, l p 2 n}(E) \equiv & \text { Cross section for producing one proton and two neutrons } \\
& \text { only.** }
\end{aligned} \\
& \sigma_{p, f r}(E) \equiv \text { Cross section for fragment production. } \\
& \begin{aligned}
\sigma_{p, f r}(E ; z>4) \equiv & \text { Cross section for fragment production with charge greater } \\
& \text { than or equal to four. }
\end{aligned} \\
& \sigma_{p, 2 P r}(E) \equiv \text { Cross section for producing two prongs. }
\end{aligned}
$$

*The example is poor. The notation is more meaningful if the incident particle is a pion, for then $\sigma_{\pi D}$ represents the pion absorption cross section.

$* * \sigma_{\pi^{+}, 1 \pi^{\circ}}$ is used at low energies to signify the $\pi^{+}$charge-exchange cross section. 
Ditiferential Cross Sections as a Function of the Incident Proton Energy and the other Variables Indicated

All variables are measured in the laboratory system.

$\sigma_{p, x p y}\left(E ; E_{p}, \psi_{p}\right) \equiv$ Cross section for producing a proton with energy $E_{p}$ at angle $\psi_{\mathrm{p}}$ by any nonelastic process $(\mathrm{mb} / \mathrm{MeV} \cdot \mathrm{sr})$.

$\sigma_{p, x p}\left(E ; E_{p}, \psi_{p}\right) \equiv$ Cross section for producing a proton with energy $E_{p}$ at angle $\theta_{p}$ by those nonelastic processes in which protons only are emitted $(\mathrm{mb} / \mathrm{MeV} \cdot \mathrm{sr})$.

$\sigma_{p, x p y}\left(E ; E_{p}\right)=\int \sigma_{p, x p y}\left(E ; E_{p} ; \Psi_{p}\right) d \Omega(m b / M e V)$.

$\sigma_{p, x p y}\left(E ; \psi_{p}\right)=\int \sigma_{p, x p y}\left(E ; E_{p}, \psi_{p}\right) d E_{p}(m b / s r)$

Note $: \int \sigma_{p, x p y}\left(E ; \psi_{p}\right) \cdot d \Omega=\int \sigma_{p, x p y}\left(E ; E_{p}\right) d E_{p}$

$=v_{\mathrm{p}} \sigma_{\mathrm{p}, \mathrm{xpy}}(\mathrm{E})$,

where $v_{p}$ is the average number of protons emitted per inelastic collision.

$\sigma_{p, 2 p}\left(E_{p 1} E_{p l}, \psi_{p l}, E_{p 2}, \psi_{p 2}\right) \equiv$ Cross section for producing two protons only, one with energy $E_{\mathrm{pl}}$ at an angle $\psi_{\mathrm{pl}}$ and the other with energy $\mathrm{E}_{\mathrm{p} 2}$ at an angle $\psi_{\mathrm{p} 2}\left[\mathrm{mb} /(\mathrm{MeV})^{2}(\mathrm{sr})^{2}\right]$. 


\section{ACKNOWLEDGMENT}

The author would like to acknowledge the hard work and conscientious efforts of Mrs. Elsie Pickell, Miss Barbara Bishop, Mrs. Jackie Gillen, and Mrs. Evelyn Ingram while assisting in all phases of this task. 
TABULATION OF REACTIONS FOR VARIOUS INCIDENT PARTICLES

\begin{tabular}{|c|c|c|c|c|c|c|c|c|c|}
\hline No. & $\begin{array}{l}\text { Incident } \\
\text { Particle }\end{array}$ & $\begin{array}{c}\text { Incident } \\
\text { Particle } \\
\text { Energy (Gev) }\end{array}$ & Target & Meäsured Quantity & $\begin{array}{l}\text { Estimate of } \\
\text { Experimental } \\
\text { Error }(\$)\end{array}$ & Detector & $\begin{array}{l}\text { Installation and } \\
\text { Accelerator }\end{array}$ & References & Comments \\
\hline 1 & $\mathrm{p}$ & 0.143 & D & $\begin{array}{l}\text { Neutron spectra at } \\
\text { zero degrees } \\
\therefore\end{array}$ & $3-10$ & $\begin{array}{l}\text { Neutron THme- } \\
\text { of-Flight } \\
\text { Spectrometer }\end{array}$ & $\begin{array}{l}\text { Harwell, } \\
\text { Synchrocyclotron }\end{array}$ & $\begin{array}{l}\text { P.H. Bowen et al., } \\
\text { Nuclear Phys. } \\
475 \text { (1962) }\end{array}$ & \\
\hline 2 & $\mathrm{p}$ & $\cdot 0.340$ & D & $\begin{array}{c}\text { Proton spectra at } \\
30^{\circ} \text { and } 40^{\circ} \\
\therefore \therefore\end{array}$ & $E<10$ & $\begin{array}{l}\text { 35-Channel } \\
\text { Magnetic } \\
\text { Particle } \\
\text { Spectrometer }\end{array}$ & $\begin{array}{l}\text { Berkeley, } \\
\text { Synchrocyclotron }\end{array}$ & $\begin{array}{l}\text { J.B. Cladis, W.N. Hess, } \\
\text { and B.J. Moyer, Phys. } \\
\text { Rev. 87, } 425 \text { (1952) }\end{array}$ & \\
\hline 3 & $\mathrm{p}$ & 0.660 & D & $\begin{array}{l}\sigma_{p, x \pi^{+} y}\left(E_{\pi^{+}}, \psi_{\pi^{+}}\right) \\
\sigma_{p, x \pi^{-} y}\left(E_{\pi^{-}}, \psi_{\pi^{-}}\right)\end{array}$ & 25 & Telescope & $\begin{array}{l}\text { Joint Institute } \\
\text { for Nuclear } \\
\text { Research, } \\
\text { Synchrocyclotron }\end{array}$ & $\begin{array}{l}\text { A.G. Meshkovskii et al., } \\
\text { Soviet Phys.-JETP } \frac{5}{5} \text {, } \\
\text { 1085 (1957) }\end{array}$ & \\
\hline 4 & $p$ & 0.910 & D & $\sigma_{p T}$ & 3 & Telescope & $\begin{array}{l}\text { Birmingham, } \\
\text { Synchrotron }\end{array}$ & $\begin{array}{l}\text { M.E. Law, G. W. Hutchinson, } \\
\text { and D.H. White, Nuclear } \\
\text { Phys. 2, } 600 \text { (1959) }\end{array}$ & \\
\hline 5 & $p$ & 0.970 & $\mathrm{Ee}$ & $\begin{array}{cl}\sigma_{p T^{\prime}} ; & \sigma_{p x} ; \\
\sigma_{p, x \pi^{+} y} ; & \sigma_{p, x \pi^{-} y}\end{array}$ & 15 & $\begin{array}{l}\text { Cloud } \\
\text { Chamber }\end{array}$ & Birmịngham & $\begin{array}{l}\text { L. Riddiford and A. } \\
\text { Williams, Proc. Roy. Soc. } \\
\text { (London) A257, } 316 \text { (1960) }\end{array}$ & \\
\hline 6 & $\mathrm{p}$ & 0.096 & $\mathrm{Li}$ & $\sigma_{p, x p y}\left(E_{p}, 40^{\circ}\right)$ & 5 & $\begin{array}{l}\text { Range } \\
\text { Telescope }\end{array}$ & $\begin{array}{l}\text { Hiarvard, } \\
\text { Cyclotron }\end{array}$ & $\begin{array}{l}\text { K. Strauch and F. Titus, } \\
\text { Phys. Rev. 104, } 191 \text { (1956) }\end{array}$ & . \\
\hline 7 & $p$ & 0.143 & Li & $\begin{array}{l}\text { Neutron spectra at } \\
\text { zero degrees }\end{array}$ & $3-10$ & $\begin{array}{l}\text { Neutron Time- } \\
\text { of-Flight } \\
\text { Spectrometer }\end{array}$ & $\begin{array}{c}\text { Harwell, } \\
\text { Synchrocyclotron }\end{array}$ & $\begin{array}{l}\text { P.H. Bowen et al., } \\
\text { Nuclear Phys. } 30 \text {,' } \\
475 \text { (1962) }\end{array}$ & \\
\hline 8 & $p$ & 0.155 & $\mathrm{Li}^{6}$ & $\begin{array}{l}\text { (p,2p) reactions. } \\
\text { Total energy spectra } \\
\text { and angular correla- } \\
\text { tion of emitted } \\
\text { protons }\end{array}$ & $5-25$ & Telescope & $\begin{array}{c}\text { Orsay, } \\
\text { Synchrocyclotron }\end{array}$ & $\begin{array}{l}\text { J.P. Garron et al al } \\
\text { Nuclear Phys. } 37 \\
\text { (1962) }\end{array}$ & \\
\hline
\end{tabular}




\begin{tabular}{|c|c|c|c|c|c|c|c|c|c|}
\hline No. & $\begin{array}{l}\text { Incident } \\
\text { Farticle }\end{array}$ & $\begin{array}{l}\text { Incident } \\
\text { Particle } \\
\text { Energy (Gev) }\end{array}$ & Target & Measured Quantity & $\begin{array}{l}\text { Estimate of } \\
\text { Experimental } \\
\text { Error }\left(\phi_{0}\right)\end{array}$ & Detector & $\begin{array}{l}\text { Installation and } \\
\text { Accelerator }\end{array}$ & References & Comments \\
\hline 9 & $\mathrm{p}$ & 0.155 & $\mathrm{Li}^{7}$ & $\begin{array}{l}\text { ( } \mathrm{p}, 2 \mathrm{p}) \text { reactions. Total } \\
\text { energy spectra and } \\
\text { angular correlation of } \\
\text { emitted protons }\end{array}$ & $5-25$ & Telescope & $\begin{array}{l}\text { Orsay, } \\
\text { Synchrocyclotron }\end{array}$ & $\begin{array}{l}\text { J. P. Garron et al., } \\
\text { Nuclear Phys. } 27 \text {. } \\
126 \text { (1962) }\end{array}$ & \\
\hline 10 & $\mathrm{p}$ & 0.180 & $\mathrm{Li}$ & $\sigma_{p X}$ & 3 & $\begin{array}{l}\text { Range } \\
\text { Separator }\end{array}$ & $\begin{array}{l}\text { Upsala, } \\
\text { Cyclotron }\end{array}$ & $\begin{array}{l}\text { A. Johansscn, U. Svanberg, } \\
\text { and O. Sundberg, Arkiv } \\
\text { Fysik 19 } 527 \text { (1961) }\end{array}$ & Poor geometry \\
\hline 11 & $\mathrm{p}$ & 0.185 & $\mathrm{Ii}$ & $\begin{array}{l}\sigma_{p, \text { xpy }}\left(E_{p}, \psi_{p}\right) \text { with } \\
\psi_{p}=5^{\circ}, 10^{\circ}, 18^{\circ}, 34^{\circ}\end{array}$ & $15-40$ & - & Upsala & $\begin{array}{l}\text { H. Tyren and Th.A.J. } \\
\text { Maris, Nuciear Phys. } \\
6,82 \text { (1958) }\end{array}$ & \\
\hline 12 & $\mathrm{p}$ & 0.240 & Li & $\begin{array}{l}\sigma_{p, x \pi^{+} y}\left(E_{\pi^{+}}, 90^{\circ}\right) \\
\sigma_{p, x \pi^{-} y}\left(E_{\pi^{-}}, 90^{\circ}\right)\end{array}$ & $10-30$ & $\begin{array}{l}\text { Scintillation } \\
\text { Crystal } \\
\text { Telescope }\end{array}$ & $\begin{array}{l}\text { Rochester, } \\
\text { Synchrocyclotron }\end{array}$ & $\begin{array}{l}\text { E.K. Gotchell, Phys, } \\
\text { Rev. 105, i13 (1957) }\end{array}$ & \\
\hline 13 & $\mathrm{p}$ & 0.660 & $\mathrm{LI}$ & $\sigma_{p, x \pi^{+} y}\left(E_{\pi^{+}}, 45^{\circ}\right)$ & 10 & $\begin{array}{l}\text { Magnetic } \\
\text { Method } \\
\text { Telescope }\end{array}$ & $\begin{array}{l}\text { Institute for } \\
\text { Nuclear Problems, } \\
\text { Synchrocyclotron }\end{array}$ & $\begin{array}{l}\text { A.G. Meshkcvskii et al., } \\
\text { Soviet Phys. JWTIP } \\
8+2 \text { (1957) }\end{array}$ & \\
\hline 14 & $\mathrm{p}$ & 0.660 & $\mathrm{Li}$ & $\sigma_{p, x \pi-y}\left(E_{\pi}-, 45^{\circ}\right)$ & 20 & - & $\begin{array}{l}\text { Joint Institute } \\
\text { for Nuclear } \\
\text { Rəsearch, } \\
\text { Synchrocyclotron }\end{array}$ & $\begin{array}{l}\text { A.G. Meshkovskii, Ia. } \\
\text { Ia. Shalamov, and V.A. } \\
\text { Shebanov, Soviet Phys.- } \\
\text { JETP 6, } 463 \text { (1958) }\end{array}$ & \\
\hline 15 & $\mathrm{p}$ & 0.096 & $\mathrm{Be}$ & $\sigma_{p, x \supseteq y}\left(E_{p}, 40^{\circ}\right)$ & 5 & $\begin{array}{l}\text { Range } \\
\text { Telescope }\end{array}$ & $\begin{array}{l}\text { Harvard } \\
\text { cyclotron }\end{array}$ & $\begin{array}{l}\text { K. Strauch and F. Titus, } \\
\text { Phys. Rev. 104, i91 (1956) }\end{array}$ & \\
\hline 16 & $\mathrm{p}$ & 0.142 & $\mathrm{Be}$ & $\sigma_{\mathrm{pT}} ; \sigma_{\mathrm{p}, \mathrm{p}}$ & $2-10$ & $\begin{array}{l}\text { Counter } \\
\text { Telescope }\end{array}$ & $\begin{array}{c}\text { Harwell, } \\
\text { Sychrocyclotron }\end{array}$ & $\begin{array}{l}\text { A.E. Taylor and E. Wood, } \\
\text { Nruclear Phys. 25, } 642 \\
\text { (1961) }\end{array}$ & Good geometry \\
\hline
\end{tabular}




\begin{tabular}{|c|c|c|c|c|c|c|c|c|c|}
\hline No. & $\begin{array}{l}\text { Incident } \\
\text { Particle }\end{array}$ & $\begin{array}{c}\text { Incident } \\
\text { Particle } \\
\text { Energy (Gev) }\end{array}$ & Target & Measured Quantity & $\begin{array}{l}\text { Estimate of } \\
\text { Experimental } \\
\text { Error }(\phi)\end{array}$ & Detector & $\begin{array}{l}\text { Installation and } \\
\text { Accelerator }\end{array}$ & References & Comments \\
\hline 17 & $p$ & 0.143 & $\mathrm{Be}$ & $\begin{array}{l}\text { Neutron spectra at } \\
\text { zero degrees }\end{array}$ & $3-10$ & $\begin{array}{l}\text { Neutron Time } \\
\text { of-Flight } \\
\text { Spectrometer }\end{array}$ & $\begin{array}{c}\text { Harwell, } \\
\text { Synchrocyclotron }\end{array}$ & $\begin{array}{l}\text { P.H. Bowen et al., } \\
\text { Nuclear Phys. } 30 \\
475 \text { (1962) }\end{array}$ & \\
\hline 18 & $\mathrm{p}$ & 0.180 & $\mathrm{Be}$ & $\sigma_{\mathrm{pX}}$ & 3 & $\begin{array}{l}\text { Range } \\
\text { Separator }\end{array}$ & $\begin{array}{l}\text { Upsala, } \\
\text { Cyclotron }\end{array}$ & $\begin{array}{l}\text { A. Johansson, U. Svanberg, } \\
\text { and } 0 . \text { Sundberg, Arkiv } \\
\text { Fysik 12 } 527 \text { (1961) }\end{array}$ & Poor geometry \\
\hline 19 & $p$ & 0.185 & $\mathrm{Be}$ & $\begin{array}{l}\sigma_{p, x p y}\left(E_{p}, \psi_{p}\right) \text { with } \\
\psi_{p}=4^{\circ}, 22^{\circ}, 30^{\circ}, 60^{\circ}\end{array}$ & $15-40$ & - & Upsala & $\begin{array}{l}\text { H. Tyrén and Th.A.J. } \\
\text { Maris, Nuclear Phys. } \\
6 \text {, } 82 \text { (1958). }\end{array}$ & \\
\hline 20 & $p$ & 0.222 & $\mathrm{Be}$ & $\begin{array}{l}\text { Radio-chemical } \\
\text { cross sections }\end{array}$ & $3-40$ & Counters & $\begin{array}{l}\text { Iiverpool, } \\
\text { Synchrocyclotron }\end{array}$ & $\begin{array}{l}\text { V. Parikh, Nuclear } \\
\text { Phys. 18, } 646 \text { (1960) }\end{array}$ & \\
\hline 21 & $\mathrm{p}$ & 0.279 & $\mathrm{Be}$ & $\begin{array}{l}\text { Radio-chemical } \\
\text { cross sections }\end{array}$ & $3-40$ & Counters & $\begin{array}{l}\text { Liverpool, } \\
\text { Symchrocyclotron }\end{array}$ & $\begin{array}{l}\text { v. Parikh, Nuclear } \\
\text { Phys. } 18,646 \text { (1960) }\end{array}$ & \\
\hline 22 & $\mathrm{p}$ & 0.314 & $\mathrm{Be}$ & $\begin{array}{l}\text { Radio-chemical } \\
\text { cross sections }\end{array}$ & $3-40$ & Counters & $\begin{array}{l}\text { İiverpool, } \\
\text { Synchrocyclotron }\end{array}$ & $\begin{array}{l}\text { V. Parikh, Nuclear } \\
\text { Phys. 18, } 646 \text { (1960) }\end{array}$ & \\
\hline 23 & $\mathrm{p}$ & 0.362 & $\mathrm{Be}$ & $\begin{array}{l}\text { Radio-chemical } \\
\text { cross sections }\end{array}$ & $3-40$ & Counters & $\begin{array}{l}\text { Liverpool, } \\
\text { Synchrocyclotron }\end{array}$ & $\begin{array}{l}\text { V. Parikh, Nuclear } \\
\text { Phys. 18, } 646 \text { (1960) }\end{array}$ & \\
\hline 24 & $\mathrm{p}$ & 0.450 & $\mathrm{Be}$ & $\begin{array}{c}\sigma_{p, x \pi^{+} y}\left(E_{\pi^{+}}, \psi_{\pi^{+}}\right) \\
\sigma_{p, x \pi^{-} y}\left(E_{\pi^{-}}, \psi_{\pi^{-}}\right) \\
\text {with } \psi_{\pi^{ \pm}}=21.5^{\circ}, 60^{\circ}\end{array}$ & $2-40$ & $\begin{array}{l}\text { Counter } \\
\text { Telescope }\end{array}$ & $\begin{array}{l}\text { Chicago, } \\
\text { Synchrocyclotron }\end{array}$ & $\begin{array}{l}\text { E. Lillethun, Phys. } \\
\text { Rev. 125, } 665 \text { (1962) }\end{array}$ & Good geometry \\
\hline 25 & $\mathrm{p}$ & $0.185-0.870$ & $\mathrm{Be}$ & $\sigma_{\mathrm{pX}}$ & 10 & - & - & $\begin{array}{l}\text { G.P. Milluburn et al., } \\
\text { Phys. Rev. 95, } \\
\text { (1954) }\end{array}$ & A compilation \\
\hline
\end{tabular}




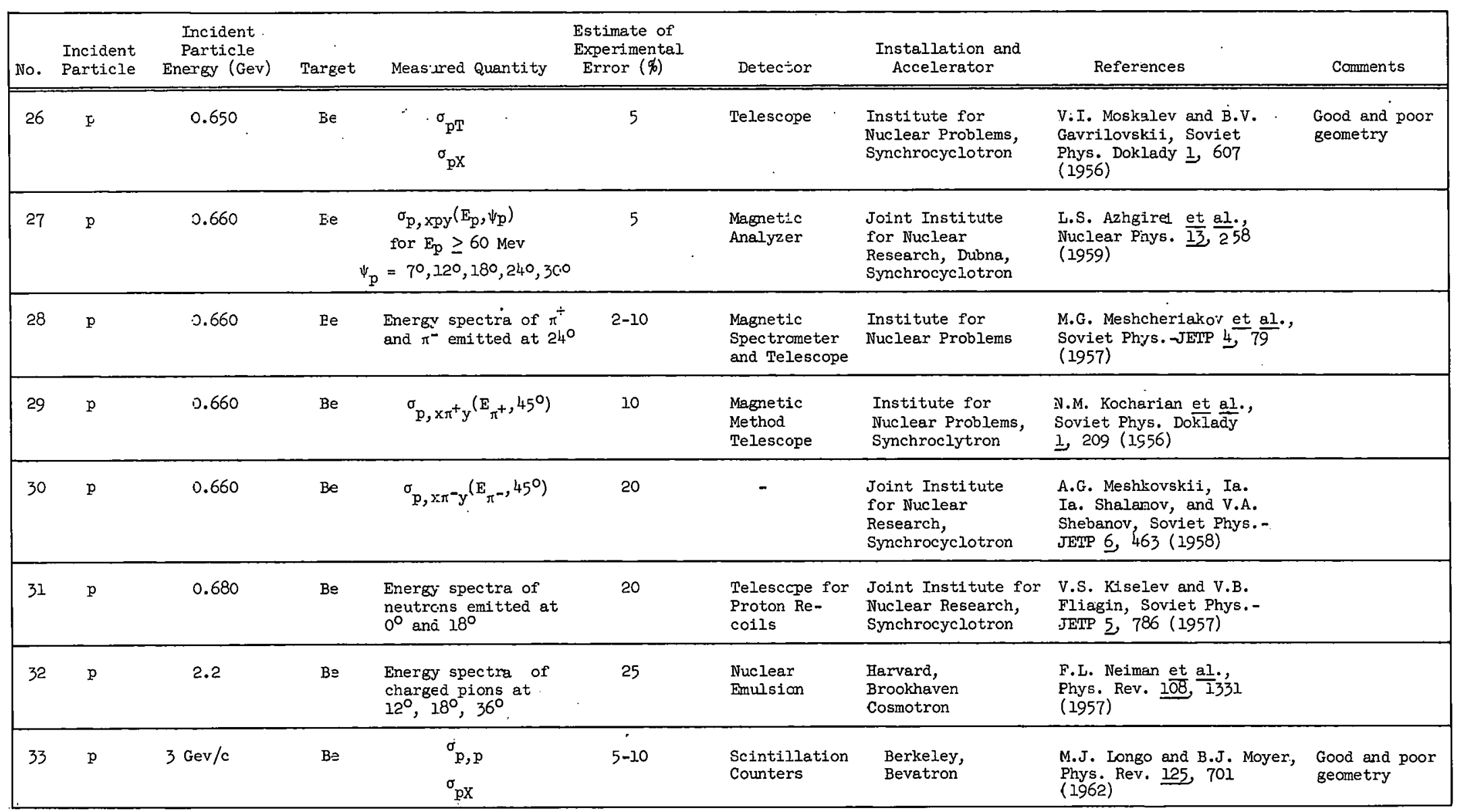




\begin{tabular}{|c|c|c|c|c|c|c|c|c|}
\hline No. & $\begin{array}{l}\text { Incident } \\
\text { Particle }\end{array}$ & $\begin{array}{c}\text { Incicient } \\
\text { Particle } \\
\text { Energy (Gev) }\end{array}$ & Terget & $\begin{array}{cc}\text { Estimate of } \\
\text { Measured Quantity } \\
\text { Experimental } \\
\text { Error }(\%)\end{array}$ & Detector & $\begin{array}{l}\text { Installation and } \\
\text { Accelerator }\end{array}$ & References & Comments \\
\hline 34 & $p$ & 5.7 & $\mathrm{Be}$ & $\begin{array}{l}\sigma_{\mathrm{p}}, \mathrm{lpln} \text { and other } \\
\text { radio-chemical } \\
\text { cross sections }\end{array}$ & NaI CrJstal & $\begin{array}{l}\text { Berkeley, } \\
\text { Bevatron }\end{array}$ & $\begin{array}{l}\text { P.A. Benioff Phys. } \\
\text { Rev. 119, } 316 \text { (1960) }\end{array}$ & \\
\hline 35 & $p$ & 10 & $\mathrm{Be} e^{\circ}$ & $\begin{array}{l}\text { Momentum spectra of } \\
\text { protons, } \pi^{+}, \pi^{-} \text {at } \\
\text { various angles from } \\
4.75^{\circ} \text { to } 20^{\circ} \text {. Produc- } \\
\text { tion ratios of } \mathrm{K}^{+} / \pi^{+}, \mathrm{K}^{-} / \pi^{-} \text {, } \\
\overline{\mathrm{p}} / \pi^{-} \text {at } 4.75^{\circ} \text { and } 9^{\circ} \text {. }\end{array}$ & $\begin{array}{l}\text { Magnetic De- } \\
\text { flectors and } \\
\text { Focusing Gas } \\
\text { Cerenkov } \\
\text { Counters }\end{array}$ & $\begin{array}{l}\text { Brookhaven, } \\
\text { Synchrotron }\end{array}$ & $\begin{array}{l}\text { W.F. Baker et al., } \\
\text { Phys. Rev. Letters } \\
\text { I } 101 \text { (1961) }\end{array}$ & \\
\hline 36 & $\mathrm{p}$ & 20 & $\mathrm{Be}$ & $\begin{array}{l}\text { Momeritum spectra of } \\
\text { protons, } \pi^{+}, \pi^{-} \text {at } \\
\text { various angles from } \\
4.75^{\circ} \text { to } 20^{\circ} \text {. Produc- } \\
\text { tion ratios of } \mathrm{K}^{+} / \pi^{+}, \mathrm{K}^{-} / \pi^{-}, \\
\overline{\mathrm{p}} / \pi^{-} \text {at } 4.75^{\circ} \text { and } 9^{\circ} \text {. }\end{array}$ & $\begin{array}{l}\text { Magnet_c De- } \\
\text { flectors and } \\
\text { Focusing Gas } \\
\text { Cerenkov } \\
\text { Counters }\end{array}$ & $\begin{array}{c}\text { Brookhaven, } \\
\text { Synchrotron } \\
\therefore\end{array}$ & $\begin{array}{l}\text { W.F. Baker et al., } \\
\text { Phys. Rev. Letters } \\
7,101 \text { (1961) }\end{array}$ & \\
\hline 37 & $\mathrm{p}$ & 25 & $\mathrm{Be}$ & $\begin{array}{l}\text { Momentum spectra of } \\
\text { protons, } \pi^{+}, \pi^{-} \text {at } \\
\text { various angles from } \\
4.75^{\circ} \text { to } 20^{\circ} \text {. Produc- } \\
\text { tion ratios of } \mathrm{K}^{+} / \pi^{+}, \mathrm{K}^{-} / \pi^{-} \text {, } \\
\overline{\mathrm{p}} / \pi^{-} \text {at } 4.75^{\circ} \text { and } 9^{\circ} \text {. }\end{array}$ & $\begin{array}{l}\text { Magnetic De- } \\
\text { flectors and } \\
\text { Focusing Gas } \\
\text { Cerenkov } \\
\text { Counters }\end{array}$ & $\begin{array}{l}\text { Brookhaven, } \\
\text { Synchrotron }\end{array}$ & $\begin{array}{l}\text { W.F. Baker et al., } \\
\text { Phys. Rev. Letters } \\
\text { I, } 101 \text { (1961) }\end{array}$ & \\
\hline 38 & $p$ & 29.5 & $\mathrm{~B} \in$ & 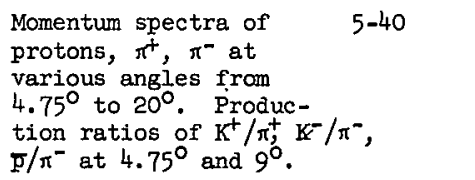 & $\begin{array}{l}\text { Magnetic De- } \\
\text { flectors and } \\
\text { Focusing Gas } \\
\text { Cerenk.ov } \\
\text { Counters }\end{array}$ & $\begin{array}{l}\text { Brookhaven, } \\
\text { Synchrotron }\end{array}$ & $\begin{array}{l}\text { W.F. Baker et al. } \\
\text { Phys. Rev. } \frac{\text { Letters }}{\text { I } 101 \text { (1961) }}\end{array}$ & . \\
\hline
\end{tabular}




\begin{tabular}{|c|c|c|c|c|c|c|c|c|c|}
\hline No. & $\begin{array}{l}\text { Incident } \\
\text { Particle }\end{array}$ & $\begin{array}{c}\text { Ircident } \\
\text { Particle } \\
\text { Energy (Gev) }\end{array}$ & Target & Measured quantity & $\begin{array}{l}\text { Estimate of } \\
\text { Experimental } \\
\text { Error }(\phi)\end{array}$ & Detector & $\begin{array}{l}\text { Installation and } \\
\text { Accelerator }\end{array}$ & References & Comments \\
\hline 39 & $p$ & $30^{\circ}$ & $\mathrm{Be}$ & $\begin{array}{l}\text { Momentum spectra of } \\
\pi^{ \pm} \text {deuterons, tritons, } \\
\mathrm{He}^{3} \text {, protons, anti- } \\
\text { protons, } \mathrm{K}^{ \pm} \text {at } 13^{\circ} \text {, } \\
45^{\circ} \text {, and } 90^{\circ}\end{array}$ & s, & $\begin{array}{l}\text { Momentum De- } \\
\text { flection and } \\
\text { Time-of-Flight }\end{array}$ & $\begin{array}{l}\text { Brookhaven, } \\
\text { Synchrotron }\end{array}$ & $\begin{array}{l}\text { v. Fitch, S. Meyer, and } \\
\text { P. Pirou'e, Phys. Rev. } \\
\text { 126, } 1849 \text { (1962) }\end{array}$ & \\
\hline 40 & $\mathrm{p}$ & 33 & $\mathrm{Be}$ & $\begin{array}{l}\text { Momentim spectra of } \\
\pi^{ \pm} \text {deuterons, tritons, } \\
\mathrm{He}^{3} \text {, Frotons, anti- } \\
\text { protors, } \mathrm{K}^{ \pm} \text {at } 13^{\circ} \text {, } \\
45^{\circ} \text {, end } 90^{\circ}\end{array}$ & s, & $\begin{array}{l}\text { Momentum De- } \\
\text { flection and } \\
\text { Time-of-Flight }\end{array}$ & $\begin{array}{l}\text { Brookhaven, } \\
\text { Synchrotron }\end{array}$ & $\begin{array}{l}\text { V. Fitch, S. Meyer, and } \\
\text { P. Piroué, Phys. Rev. } \\
126,1849 \text { (1962) }\end{array}$ & \\
\hline 41 & $\mathrm{p}$ & 0.096 & $\mathrm{~g}$ & $\sigma_{p, x p y}\left(E_{p}, 40^{\circ}\right)$ & 5 & $\begin{array}{l}\text { Range } \\
\text { Telescope }\end{array}$ & $\begin{array}{l}\text { Harvard, } \\
\text { Cyclotron }\end{array}$ & $\begin{array}{l}\text { K. Strauch and F. Titus, } \\
\text { Phys. Rev. 104 191 } \\
\text { (1956) }\end{array}$ & \\
\hline 42 & $\mathrm{p}$ & 0.155 & $\mathrm{~B}^{10}$ & $\begin{array}{l}\text { ( } p, 2 p) \text { reactions. } \\
\text { Total energy spectra } \\
\text { and angular correla- } \\
\text { tion of enitted protons }\end{array}$ & ns & Telescope & $\begin{array}{c}\text { Orsay, } \\
\text { Synchrocyclotron }\end{array}$ & $\begin{array}{l}\text { J.P. Garron et al., } \\
\text { Nuclear Phys. } 37 \\
126 \text { (1962) }\end{array}$ & \\
\hline 43 & $\mathrm{p}$ & 0.155 & $\mathrm{~B}^{11}$ & $\begin{array}{l}(p, 2 p) \text { reactions. } \\
\text { Total energy spectra } \\
\text { and angular correla- } \\
\text { tion cf enitted protons }\end{array}$ & ns & Telescope & $\begin{array}{c}\text { Orsay, } \\
\text { Synchrocyclotron }\end{array}$ & $\begin{array}{l}\text { J.P. Garron et al., } \\
\text { Nuclear Phys. } 3 \frac{17}{126 \text { (1962) }}\end{array}$ & \\
\hline+4 & $\mathrm{p}$ & 0.185 & $B$ & $\begin{array}{c}\sigma_{p}, x p y\left(E_{p}, \psi_{p}\right) \text { for } \\
\psi_{p}=5^{\circ}, 14^{\circ}, 18^{\circ}, 23^{\circ}\end{array}$ & $15-40$ & - & Upsala & $\begin{array}{l}\text { H. Tyrén and Th.A.J. } \\
\text { Maris, Nuclear Phys. } \\
5 \text { S } 82 \text { (1958) }\end{array}$ & \\
\hline 45 & $\mathrm{p}$ & 0.061 & $\mathrm{C}$ & $\begin{array}{l}\sigma_{\mathrm{p} T} \\
\sigma_{\mathrm{pX}}\end{array}$ & 6 & $\begin{array}{l}\text { Plastic } \\
\text { Scintillators }\end{array}$ & Minnesota & $\begin{array}{l}\text { V. Meyer, R.M. Eisberg, } \\
\text { and R.F. Carlson, Phys. } \\
\text { Rev. } 117 \text {, } 1334 \text { (1960) }\end{array}$ & \\
\hline
\end{tabular}




\begin{tabular}{|c|c|c|c|c|c|c|c|c|c|}
\hline No. & $\begin{array}{l}\text { Incident } \\
\text { Particle }\end{array}$ & $\begin{array}{c}\text { Incident } \\
\text { Particle } \\
\text { Energy (Gev) }\end{array}$ & Target & Measured Quantity & $\begin{array}{l}\text { Estimate of } \\
\text { Experimental } \\
\text { Error }(\phi)\end{array}$ & Detector & $\begin{array}{l}\text { Installation and } \\
\text { Accelerator }\end{array}$ & References & Comments \\
\hline 46 & $\mathrm{p}$ & 0.077 & $\mathrm{C}$ & $\sigma_{\mathrm{pX}}$ & $\sim 3$ & $\begin{array}{l}\text { Range } \\
\text { Telescope }\end{array}$ & $\begin{array}{l}\text { Harvarà, } \\
\text { Cyclotron }\end{array}$ & $\begin{array}{l}\text { R. Goloskie and K. } \\
\text { Strauch, Nuclear } \\
\text { Phys. 29, } 474 \text { (1962) }\end{array}$ & Good geometry \\
\hline 47 & $p$ & 0.095 & $\mathrm{c}$ & $\sigma_{\mathrm{pX}}$ & $\sim 3$ & $\begin{array}{l}\text { Range } \\
\text { Telescope }\end{array}$ & $\begin{array}{l}\text { Harvara, } \\
\text { Cyclotron. }\end{array}$ & $\begin{array}{l}\text { R. Goloskie and K. } \\
\text { Strauch, Nuclear } \\
\text { Phys. 29, } 474 \text { (1962) }\end{array}$ & Good geometry \\
\hline 48 & $p$ & 0.096 & c & $\sigma_{p, x p y}\left(E_{p}, 40^{\circ}\right)$ & 5 & $\begin{array}{l}\text { Range } \\
\text { Telescope }\end{array}$ & $\begin{array}{l}\text { Harvard, } \\
\text { Cyclotron }\end{array}$ & $\begin{array}{l}\text { K. Strauch and F. Titus, } \\
\text { Phys. Rev. 104, } 191 . \\
\text { (1956) }\end{array}$ & \\
\hline 49 & $\mathrm{p}$ & 0.113 & c & $\sigma_{\mathrm{pX}}$ & $\sim 3$ & $\begin{array}{l}\text { Range } \\
\text { Telescope }\end{array}$ & $\begin{array}{l}\text { Harvard, } \\
\text { Cyclotron }\end{array}$ & $\begin{array}{l}\text { R. Goloskie and } k \text {. } \\
\text { Strauch, Nuclear } \\
\text { Phys. 29, } 474 \text { (1962) }\end{array}$ & Good geometry \\
\hline 50 & $\mathrm{p}$ & 0.133 & c & $\sigma_{\mathrm{pX}}$ & $\sim 3$ & $\begin{array}{l}\text { Range } \\
\text { Telescope }\end{array}$ & $\begin{array}{l}\text { Harvard, } \\
\text { Cyclotron }\end{array}$ & $\begin{array}{l}\text { R. Goloskie and K. } \\
\text { Strauch, Nuclear } \\
\text { Phys. 29, } 474 \text { (1962) }\end{array}$ & Good geometry \\
\hline 51 & $\mathrm{p}$ & 0.142 & $\mathrm{C}$ & $\begin{array}{l}\sigma_{p T} \\
\sigma_{p, p}\end{array}$ & $2-10$ & $\begin{array}{l}\text { Counter } \\
\text { Telescope }\end{array}$ & $\begin{array}{c}\text { Harwell, } \\
\text { Synchrocyclotron }\end{array}$ & $\begin{array}{l}\text { A.E. Taylor and E. } \\
\text { Wood, Nuclear Phys. } \\
\text { 25, } 642(1961)\end{array}$ & Good geometry \\
\hline 52 & $\mathrm{p}$ & 0.143 & $\mathrm{C}$ & $\begin{array}{l}\text { Neutron spectra } \\
\text { at zero cegrees }\end{array}$ & $3-10$ & $\begin{array}{l}\text { Neutron Time- } \\
\text { of-Flight } \\
\text { Spectrometer }\end{array}$ & $\begin{array}{c}\text { Harwell, } \\
\text { Symchrocyclotron }\end{array}$ & $\begin{array}{l}\text { P.H. Bowen et al., } \\
\text { Nuclear Phys. 30, } \\
475 \text { (1962) }\end{array}$ & \\
\hline 53 & $\mathrm{p}$ & 0.150 & c & $\begin{array}{l}\text { Tritium production } \\
\text { cross section }\end{array}$ & $\sim 10$ & $\begin{array}{l}\text { Radio-Chemical } \\
\text { Methods }\end{array}$ & $\begin{array}{c}\text { Orsay, } \\
\text { Synchrocyclotron }\end{array}$ & $\begin{array}{l}\text { C. Brun, M. Lefort, and } \\
\text { X. Tarrago, J. phys. } \\
\text { radium 23, } 167 \text { (1962) }\end{array}$ & \\
\hline
\end{tabular}




\begin{tabular}{|c|c|c|c|c|c|c|c|c|c|}
\hline No. & $\begin{array}{l}\text { Incident } \\
\text { Particle }\end{array}$ & $\begin{array}{c}\text { Incident } \\
\text { Particle } \\
\text { Energy (Gev) }\end{array}$ & Target & Measured Quantity & $\begin{array}{l}\text { Estimate of } \\
\text { Experimental } \\
\text { Error }(\phi)\end{array}$ & Detector & $\begin{array}{l}\text { Installation and } \\
\text { Accelerator }\end{array}$ & References & Comments \\
\hline 54 & $\mathrm{p}$ & 0.150 & C & $\sigma_{p, I p I n}$ & 5 & - & $\begin{array}{l}\text { Institute for } \\
\text { Nuclear Problems }\end{array}$ & $\begin{array}{l}\text { Yu. D. Prokoshkin and } \\
\text { A.A. Tiapkin, Soviet } \\
\text { Fhys. JETP 5, } 148 \\
\text { (1957) }\end{array}$ & . \\
\hline 55 & $\mathrm{p}$ & 0.153 & $\mathrm{C}^{12}$ & $\begin{array}{l}\text { Cross sections for } \\
\text { producing excited } \\
\text { states in ( } p, 2 p) \\
\text { and }(p, n p) \text { reactions }\end{array}$ & $20-50$ & $\begin{array}{l}\text { Coincidence } \\
\text { Counters and } \\
\text { NaI Crystals }\end{array}$ & $\begin{array}{l}\text { Harwell, } \\
\text { Cyclotron }\end{array}$ & $\begin{array}{l}\text { s. Austin et al., } \\
\text { Putherford Jubilee } \\
\text { Conf. Manciester, } \\
\text { 1961, Paper C/l, p. } 139\end{array}$ & Poor geometry \\
\hline 56 & $p$ & 0.153 & c & $\begin{array}{l}\sigma_{\mathrm{p}, 1 p l n} ; \sigma_{\mathrm{p}, 2 \mathrm{p}} \text { for } \\
\text { various excited final } \\
\text { states }\end{array}$ & $20-30$ & $\begin{array}{l}\text { Counter } \\
\text { Telescope } \\
\text { and NaI } \\
\text { Crystal }\end{array}$ & $\begin{array}{l}\text { Oxford Atomic } \\
\text { Energy Research } \\
\text { Establishment, } \\
\text { Cyclotron }\end{array}$ & $\begin{array}{l}\text { S.M. Austin et al., } \\
\text { Proc. Phys. Soc..(London) } \\
\text { 80, } 383 \text { (1962) }\end{array}$ & - \\
\hline 57 & p & 0.153 & $\mathrm{C}$ & $\begin{array}{l}\text { Differential cross } \\
\text { sections for the } \\
\text { emission of } 2 \\
\text { protons at various } \\
\text { angles }\end{array}$ & 20 & $\begin{array}{l}\text { Total } \\
\text { Energy } \\
\text { Scintillators }\end{array}$ & $\begin{array}{c}\text { Harwell, } \\
\text { Synchrocyclotron }\end{array}$ & $\begin{array}{l}\text { T.J. Gooding and H.G. } \\
\text { Pugh, Nuclear Phys. } \\
\text {-8 } 46 \text { (1950) }\end{array}$ & . \\
\hline 58 & $p$ & c.155 & $\mathrm{C}^{12}$ & $\begin{array}{l}\text { ( } p, 2 p) \text { reactions. } \\
\text { Total energy spectra } \\
\text { and angular correla- } \\
\text { tion of emitted protons }\end{array}$ & ns & Telescopes & $\begin{array}{l}\text { Orsay, } \\
\text { Synchrocyclotron }\end{array}$ & $\begin{array}{l}\text { J.P. Garron et al., } \\
\text { Nuclear Phys. } 37 \\
126 \text { (1962) }\end{array}$ & \\
\hline 59 & $p$ & 0.180 & $\mathrm{c}$ & $\sigma_{p X}$ & 3 & $\begin{array}{l}\text { Range } \\
\text { Separator }\end{array}$ & Upsala & $\begin{array}{l}\text { A. Johansson, U. } \\
\text { Svanberg, and O. } \\
\text { Sundberg, Arkiv Fysik } \\
\text { 19, } 527 \text { (1961) }\end{array}$ & Poor geometry \\
\hline
\end{tabular}




\begin{tabular}{|c|c|c|c|c|c|c|c|c|c|}
\hline No. & $\begin{array}{l}\text { Incident } \\
\text { Particle }\end{array}$ & $\begin{array}{c}\text { Incident } \\
\text { Particle } \\
\text { Energy (Gev) }\end{array}$ & Target & Measured Quantity & $\begin{array}{l}\text { Estimate of } \\
\text { Experimental } \\
\text { Error }(\%)\end{array}$ & Detector & $\begin{array}{l}\text { Installation and } \\
\text { Accelerator }\end{array}$ & References & Comments \\
\hline 60 & $\mathrm{p}$ & 0.185 & $\mathrm{c}$ & $\begin{array}{l}\text { Nuclear shell struc- } \\
\text { ture from } \\
\sigma_{p ; 2 p}\left(E_{p 1}, E_{p 2}, \psi_{p 1}, \psi_{p 2}\right)\end{array}$ & 10 & $\begin{array}{l}\text { Double Tele- } \\
\text { scope Range } \\
\text { Method for } \\
\text { Energi }\end{array}$ & Upsala & $\begin{array}{l}\text { H. Tyrén, P. Hillman, } \\
\text { and Th.A.J. Maris, } \\
\text { Nuclear Phys. I 10 } \\
\text { (1958) }\end{array}$ & \\
\hline $6 \bar{I}$ & $\mathrm{p}$ & 0.225 & $\mathrm{c}$ & $\begin{array}{l}\text { Radio-chemical } \\
\text { cross sections }\end{array}$ & 8 & $\begin{array}{l}\text { Radio-Chemical } \\
\text { Techniques }\end{array}$ & $\begin{array}{l}\text { Berkeley, } \\
\text { Cyclotron }\end{array}$ & $\begin{array}{l}\text { M. Honda and D. Lal, } \\
\text { Phys. Rev. 118, } 1618 \\
(1960)\end{array}$ & \\
\hline 62 & $\mathrm{p}$ & 0.240 & $\mathrm{c}$ & $\sigma_{p, x p y}\left(E_{p}, 90^{\circ}\right)$ & 10 & Emulsions & $\begin{array}{c}\text { Rochester, } \\
\text { Synchrocyclotron }\end{array}$ & $\begin{array}{l}\text { G.M. Tenmer, Phys. } \\
\text { Rev. } 83,1067 \text { (1951) }\end{array}$ & \\
\hline 63 & $\mathrm{p}$ & 0.260 & $\mathrm{c}$ & $\sigma_{p, l p l n}$ & 5 & - & $\begin{array}{l}\text { Institute for } \\
\text { Nuclear Problems }\end{array}$ & $\begin{array}{l}\text { Yu.D. Prokoshkin and } \\
\text { A.A. Tiapkin, Soviet } \\
\text { Phys. JETP 5, } 148 \\
\text { (1957) }\end{array}$ & \\
\hline 64 & $\mathrm{p}$ & 0.202 to 0.342 & C & $\begin{array}{l}\sigma p, 3 p 3 n \text { relative to } \\
\sigma \text { of } c^{12}(p, p n) c^{11}\end{array}$ & $2-10$ & Counters & $\begin{array}{l}\text { Liverpool, } \\
\text { Synchrocyclotron }\end{array}$ & $\begin{array}{l}\text { V. Parikh, Nuclear } \\
\text { Phys. 18, } 638 \text { (1960) }\end{array}$ & \\
\hline 65 & $\mathrm{p}$ & 0.288 & $\mathrm{C}$ & $\sigma_{p}, \operatorname{lp} \ln$ & 3 & $\begin{array}{l}\text { Ganma-Ray } \\
\text { Counter }\end{array}$ & $\begin{array}{l}\text { Liverpool, } \\
\text { Synchrocyclotron }\end{array}$ & $\begin{array}{l}\text { V. Parikh, Nuclear } \\
\text { Phys. 18, } 628 \text { (1960) }\end{array}$ & \\
\hline 66 & $\mathrm{p}$. & 0.300 & c & $\begin{array}{l}\text { Radio-chemical } \\
\text { cross sections }\end{array}$ & 8 & $\begin{array}{l}\text { Radio-Ohemical } \\
\text { Techniques }\end{array}$ & $\begin{array}{l}\text { Berkeley, } \\
\text { Cyclotron }\end{array}$ & $\begin{array}{l}\text { M. Honda and D. Lal, } \\
\text { Phys. Rev. 118, } 1618 \\
(1960)\end{array}$ & \\
\hline 67 & $\mathrm{p}$ & $0.100-0.500$ & c & $\begin{array}{l}\text { Distribution of } \\
\text { fast prongs. Angular } \\
\text { distribution between } \\
2 \text { secondaries }\end{array}$ & $5-40$ & $\begin{array}{l}\text { Cloud } \\
\text { Chamber }\end{array}$ & $\begin{array}{c}\text { Milan } \\
\text { (Cosmic Rays) }\end{array}$ & $\begin{array}{l}\text { E. Fiorini and s. Ratti, } \\
\text { Nuovo cimento 14, } 901 \\
\text { (1959) }\end{array}$ & . \\
\hline
\end{tabular}




\begin{tabular}{|c|c|c|c|c|c|c|c|c|c|}
\hline No. & $\begin{array}{l}\text { Incidert } \\
\text { Particle }\end{array}$ & $\begin{array}{c}\text { Incident } \\
\text { Perticle } \\
\text { Energy (Gev) }\end{array}$ & Target & Measured Quantity & $\begin{array}{l}\text { Estimate of } \\
\text { Experimental } \\
\text { Error (क) }\end{array}$ & Detector & $\begin{array}{l}\text { Installation and } \\
\text { Accelerator }\end{array}$ & References & Comments \\
\hline 68 & $\mathrm{p}$ & 0.313 & $\mathrm{C}$ & $\sigma_{p, 1 p l n}$ & 3 & $\begin{array}{l}\text { Gamma-Ray } \\
\text { Counter }\end{array}$ & $\begin{array}{l}\text { Liverpool, } \\
\text { Syncyrocyclotron }\end{array}$ & $\begin{array}{l}\text { 7. Parikh, Nuclear } \\
\text { Phys. } 18,628(1960)\end{array}$ & \\
\hline 69 & $\mathrm{p}$ & 0.339 & $\mathrm{c}$ & $\sigma_{\mathrm{p}, \operatorname{lpln}}$ & 3 & $\begin{array}{l}\text { Gamma-Ray } \\
\text { Counter }\end{array}$ & $\begin{array}{l}\text { Liverpool, } \\
\text { Synchrocyclotron }\end{array}$ & $\begin{array}{l}\text { 7. Parikh, Nuclear } \\
\text { ?hys. 18, } 628(1960)\end{array}$ & \\
\hline io & $\mathrm{p}$ & 0.340 & $\mathrm{c}$ & $\begin{array}{l}\text { Proton spectra at } \\
30^{\circ} \text { and } 40^{\circ}\end{array}$ & $<10$ & $\begin{array}{l}\text { 35-Channel } \\
\text { Magnetic } \\
\text { Particle } \\
\text { Spectrometer }\end{array}$ & $\begin{array}{c}\text { Berkeley, } \\
\text { Symchrocyclotron }\end{array}$ & $\begin{array}{l}\text { J.B. Cladis, W.N. Hess, } \\
\text { and B.J. Noyer, Phys. } \\
\text { Rev. 87, } 425 \text { (1952) }\end{array}$ & \\
\hline$i 1$ & $p$ & 0.350 & $\mathrm{C}$ & $\sigma_{2}$, lpln & 5 & - & $\begin{array}{l}\text { Institute for } \\
\text { Nuclear Problems }\end{array}$ & $\begin{array}{l}\text { Yu.D. Prokoshkin and } \\
\text { A.A. Tiapkin, Soviet } \\
\text { Phys. JWTP 5, } 148 \\
\text { (1957) }\end{array}$ & \\
\hline 72 & $\mathrm{p}$ & 0.362 & $\mathrm{c}$ & $\sigma_{p, 1 p \ln }$ & 3 & $\begin{array}{l}\text { Gamma-Ray } \\
\text { Counter }\end{array}$ & $\begin{array}{l}\text { Liverpool, } \\
\text { Synchrocyclotron }\end{array}$ & $\begin{array}{l}\text { V. Parikh, Nuclear } \\
\text { Phys. } 18,528(1960)\end{array}$ & \\
\hline 73 & $\mathrm{p}$ & 0.383 & $\mathrm{C}$ & $\sigma_{p, I p \ln }$ & 3 & $\begin{array}{l}\text { Gamma-Ray } \\
\text { Counter }\end{array}$ & $\begin{array}{l}\text { Liverpool, } \\
\text { Synchrocyclotron }\end{array}$ & $\begin{array}{l}\text { v. Parikh, Nuclear } \\
\text { Phys. 18, } 628 \text { (1960) }\end{array}$ & \\
\hline 74 & $\mathrm{p}$ & 0.400 & $\mathrm{c}$ & $\begin{array}{l}\text { Radio-chemical } \\
\text { cross sections }\end{array}$ & 8 & $\begin{array}{l}\text { Radio-Chemical } \\
\text { Techniques }\end{array}$ & $\begin{array}{l}\text { Berkeley, } \\
\text { Cyclotron }\end{array}$ & $\begin{array}{l}\text { M. Honda and D. Lal, } \\
\text { Phys. Rev. 118, } 1618 \\
\text { (1960) }\end{array}$ & \\
\hline 75 & $p$ & 0.420 & $\mathrm{C}$ & $\begin{array}{l}\text { Radio-chemical } \\
\text { cross sections }\end{array}$ & $7-30$ & - & $\begin{array}{l}\text { Birmingham, } \\
\text { Synchrotron }\end{array}$ & $\begin{array}{l}\text { ¿.L. Symonds, J. Warren, } \\
\text { and J.D. Young, Proc. } \\
\text { Phys Soc. A(Iondon) } \\
\text { iO } 824 \text { (1957) }\end{array}$ & \\
\hline
\end{tabular}




\begin{tabular}{|c|c|c|c|c|c|c|c|c|c|}
\hline No. & $\begin{array}{l}\text { Incident } \\
\text { Particle }\end{array}$ & $\begin{array}{l}\text { Incident } \\
\text { Particle } \\
\text { Energy (Gev) }\end{array}$ & Target & Measured Quantity & $\begin{array}{l}\text { Estimate of } \\
\text { Experimental } \\
\text { Error }(\%)\end{array}$ & Detector & $\begin{array}{l}\text { Installation and } \\
\text { Accelerator }\end{array}$ & References & Comments \\
\hline 76 & $\mathrm{p}$ & 0.450 & $\mathrm{C}$ & $\begin{array}{c}\sigma_{p, x \pi^{+} y}\left(E_{\pi^{+}}, \psi_{\pi^{+}}\right) \\
\sigma_{p, x \pi^{-} y}\left(E_{\pi^{-}}, \psi_{\pi^{-}}\right) \\
\text {with } \Downarrow_{\pi^{+}}=21.5^{\circ}, 60^{\circ}\end{array}$ & $2-40$ & $\begin{array}{l}\text { Counter } \\
\text { Telescope }\end{array}$ & $\begin{array}{l}\text { Chicago, } \\
\text { Synchrocyclotron }\end{array}$ & $\begin{array}{l}\text { E. Lillethun, Phys. } \\
\text { Rev. 125, } 665 \text { (1962) }\end{array}$ & Good geometry \\
\hline 77 & $\mathrm{p}$ & 0.450 & $\mathrm{C}$ & $\sigma_{p, l p l n}$ & 5 & - & $\begin{array}{l}\text { Institute for } \\
\text { Nuclear Problems }\end{array}$ & $\begin{array}{l}\text { Yu.D. Prokoshkin and A.A. } \\
\text { Tiapkin, Soviet Phys. JETP } \\
\text { 5, } 148(1957)\end{array}$ & \\
\hline 78 & $\mathrm{p}$ & 0.470 & $\mathrm{C}$ & $\begin{array}{l}\text { Energy spectra and } \\
\text { angular distribution } \\
\text { of } \pi^{\circ}\end{array}$ & - & $\begin{array}{l}\text { Pair Gamma } \\
\text { Spectrometer }\end{array}$ & $\begin{array}{l}\text { Joint Institute } \\
\text { for Nuclear } \\
\text { Research, } \\
\text { Synchrocyclotron }\end{array}$ & $\begin{array}{l}\text { Iu.D. Baiukov; M.S. . } \\
\text { Kozodaev, and A.A. } \\
\text { Tiapkin, Soviet Phys.- } \\
\text { JETP } 5 \text {, } 552 \text { (1957) }\end{array}$ & \\
\hline 79 & $\mathrm{p}$ & 0.522 & $\mathrm{C}$ & $\begin{array}{l}\text { Radio-chenical } \\
\text { cross sections }\end{array}$ & $7-30$ & - & $\begin{array}{l}\text { Birmingham, } \\
\text { Synchrotron }\end{array}$ & $\begin{array}{l}\text { J.L. Symonds, J. Warren, and } \\
\text { J.D. Young, Proc. Phys. Soc. } \\
\text { A(London) TO, } 824 \text { (1957) }\end{array}$ & 1 \\
\hline 80 & $p$ & $0.185-0.870$ & $\mathrm{c}$ & $\sigma_{p X}$ & 10 & - & - & $\begin{array}{l}\text { G.P. Millburn et al al.' } \\
\text { Phys. Rev. } 95, \frac{1}{1268}{ }^{2}(1954)\end{array}$ & A compilation \\
\hline 81 & $\mathrm{p}$ & 0.560 & C & $\sigma_{p, l p l n}$ & 5 & - & $\begin{array}{l}\text { Institute for } \\
\text { Nuclear Problems }\end{array}$ & $\begin{array}{l}\text { Yu.D. Prokoshkin and } \\
\text { A.A. Tiapkin, Soviet Phys. - } \\
\text { JETP 5, } 148 \text { (1957) }\end{array}$ & \\
\hline 82 & $\mathrm{p}$ & 0.591 & $\mathrm{C}$ & $\sigma_{p, I p l n}$ & 5 & $\begin{array}{l}\text { Radio-Chemical } \\
\text { Methods }\end{array}$ & $\begin{array}{l}\text { CERN, } \\
\text { Scychrocyclotron }\end{array}$ & $\begin{array}{l}\text { K. Goebel, et al., Nuclear } \\
\text { Phys. } 24,28(1961)\end{array}$ & \\
\hline 83 & $\mathrm{p}$ & 0.648 & C & $\begin{array}{l}\text { Radio-chemical } \\
\text { cross sections }\end{array}$ & $7-30$ & - & $\begin{array}{l}\text { Birmingham, } \\
\text { Synchrotron }\end{array}$ & $\begin{array}{l}\text { J.L. Symonds, J. Warren, } \\
\text { and J.D. Young, Proc. Phys. } \\
\text { Soc. A(London) } 70,824 \text { (1957) }\end{array}$ & \\
\hline
\end{tabular}




\begin{tabular}{|c|c|c|c|c|c|c|c|c|c|}
\hline No. & $\begin{array}{l}\text { Incident } \\
\text { Particle }\end{array}$ & $\begin{array}{c}\text { Incident } \\
\text { Particle } \\
\text { Energy (Gev) }\end{array}$ & Target & Mecsured Quantity & $\begin{array}{l}\text { Estimate of } \\
\text { Experimental } \\
\text { Error }(\%)\end{array}$ & Detector & $\begin{array}{l}\text { Installation and } \\
\text { Accelerator }\end{array}$ & References & Comments \\
\hline 84 & $\mathrm{p}$ & 0.650 & c & $\begin{array}{l}\sigma_{\mathrm{pT}} \\
\sigma_{\mathrm{pX}}\end{array}$ & 5 & Telescope & $\begin{array}{l}\text { Institute for } \\
\text { Nuclear Problems, } \\
\text { Synchrocyclotron }\end{array}$ & $\begin{array}{l}\text { V.I. Moskalev and B.V. } \\
\text { Gavrilovskii, Soviet } \\
\text { Phys. Dcklady } 1,607 \\
\text { (1956) }\end{array}$ & $\begin{array}{l}\text { Good and poor } \\
\text { geometry }\end{array}$ \\
\hline 85 & $\mathrm{p}$ & 0.660 & c & $\begin{array}{c}a_{p, x p y}\left(E_{p}, \psi_{p}\right) \\
\text { for } E_{p}>60 \mathrm{Mev} \text { with } \\
\Psi_{p}=7^{3}, 12^{\circ}, 18^{\circ}, 24^{\circ}, 30^{\circ}\end{array}$ & 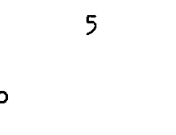 & $\begin{array}{l}\text { Magne tic } \\
\text { Analyzer }\end{array}$ & $\begin{array}{l}\text { Joint Institute } \\
\text { for Nuclear } \\
\text { Research, Dubna, } \\
\text { Synchrocyclotron }\end{array}$ & $\begin{array}{l}\text { L.s. Azhgirei et al.', } \\
\text { Nuclear Phys. } \frac{13}{258} \\
\text { (1959) }\end{array}$ & \\
\hline 86 & p. & 0.660 & $\approx$ & $\begin{array}{l}\text { EnerEy spectra of } \pi^{+} \\
\text {and } \pi^{-} \text {emitted at } 24^{\circ}\end{array}$ & $2-10$ & $\begin{array}{l}\text { Magnetic } \\
\text { Spectrometer } \\
\text { and } \\
\text { Telescope }\end{array}$ & $\begin{array}{l}\text { Institute for } \\
\text { Nuclear Problems }\end{array}$ & $\begin{array}{l}\text { M.G. Meshcheriakov et al. } \\
\text { Soviet Phys. -JETP } 4,79 \text {. } \\
\text { (1957) }\end{array}$ & \\
\hline 87 & $p$ & 0.660 & c & $\sigma_{p, x \pi^{+} y}\left(E_{\pi^{+}}, 45^{\circ}\right)$ & 10 & $\begin{array}{l}\text { Magnetic } \\
\text { Method } \\
\text { Telescope }\end{array}$ & $\begin{array}{l}\text { Institute for } \\
\text { Nuclear Problems, } \\
\text { Symchrocyclotron }\end{array}$ & $\begin{array}{l}\text { A.G. Meshkovskii et al., } \\
\text { Soviet Phys. vETP } \frac{\text { al }}{4} \\
842 \text { (1957) }\end{array}$ & \\
\hline 89 & $p$ & 0.660 & $\mathrm{C}$ & $\begin{array}{c}\sigma_{p, x \pi^{+}, y}\left(E_{\pi^{+}}, \psi_{\pi^{+}}\right) . \\
\Psi_{\pi^{+}}=20^{\circ}, 29^{\circ}, 38^{\circ}, 560,65^{\circ} \\
\sigma_{p, x \pi^{+} y}\left(\psi_{\pi^{+}}\right)\end{array}$ & 50 & - & $\begin{array}{l}\text { Jöint Institute } \\
\text { for Nuclear } \\
\text { Research, } \\
\text { Synchrocyclotron }\end{array}$ & $\begin{array}{l}\text { A.G. Meshrovskii, Ia. } \\
\text { Ia. Shalamov, and V.A. } \\
\text { Shebanov, Soviet Phys.- } \\
\text { JETP } 7,987 \text { (1958) }\end{array}$ & \\
\hline 90 & $p$ & 0.660 & $\mathrm{c}$ & $\begin{array}{l}\text { Black track number } \\
\text { distrioution. Proton } \\
\text { and alpha energy dis- } \\
\text { tribution and angular } \\
\text { distribution }\end{array}$ & $\sim 10-25$ & $\begin{array}{l}\text { Sandwica } \\
\text { Emulsions }\end{array}$ & $\begin{array}{l}\text { Radium Institute; } \\
\text { Exposure Made at } \\
\text { Joint Institute for } \\
\text { Juclear Research } \\
\text { Synchrocyclotron }\end{array}$ & $\begin{array}{l}\text { IN.A. Perfilov and Yu.I. } \\
\text { Serebrennikov, Soviet } \\
\text { Phys. JEIP 13, 274 (1961) }\end{array}$ & \\
\hline
\end{tabular}




\begin{tabular}{|c|c|c|c|c|c|c|c|c|c|}
\hline No. & $\begin{array}{l}\text { Incident } \\
\text { Particle }\end{array}$ & $\begin{array}{c}\text { Incident } \\
\text { Particle } \\
\text { Enersy (Gev) }\end{array}$ & Target & Measured Quantity & $\begin{array}{l}\text { Estimate of } \\
\text { Experimental } \\
\text { Error }(\%)\end{array}$ & Detector & $\begin{array}{l}\text { Installation and } \\
\text { Accelerator }\end{array}$ & References & Comments \\
\hline 91 & $\mathrm{p}$ & 0.660 & C & $\sigma_{p, 1 p l n}$ & 5 & - & $\begin{array}{l}\text { Institute for } \\
\text { Nuclear Problems }\end{array}$ & $\begin{array}{l}\text { Yu.D. Prokoshkin and } \\
\text { A.A. Tiapkin, Soviet } \\
\text { Phys. JETP 5, } 148 \\
\text { (1957) }\end{array}$ & \\
\hline 92 & $\mathrm{p}$ & 0.660 & $\mathrm{C}$ & $\begin{array}{l}\text { Cross section for } \\
\text { various reactions. } \\
\text { Energy and angular } \\
\text { distribution of } \\
\text { alphas and protons }\end{array}$ & $10-50$ & Emulsion & $\begin{array}{l}\text { Joint Institute for } \\
\text { Nuclear Research, } \\
\text { Synchrotron }\end{array}$ & $\begin{array}{l}\text { A.P. Zhdanov and P.I. } \\
\text { Fedotov, Soviet Phys.- } \\
\text { JETP 10, } 280 \text { (1960) }\end{array}$ & . \\
\hline 93 & $\mathrm{p}$ & 0.670 & $\mathrm{c}$ & $\begin{array}{c}\sigma_{\mathrm{p}, \mathrm{x} \pi^{+} \mathrm{y}}\left(\mathrm{E}_{\pi^{+}}, 56^{\circ}\right) \\
\sigma_{\mathrm{p}, \mathrm{x} \pi^{+} \mathrm{y}} \\
\sigma_{\mathrm{p}, \mathrm{x} \pi-\mathrm{y}}\left(\mathrm{E}_{\pi^{-}}-, 56^{\circ}\right) \\
\sigma_{\mathrm{p}, \mathrm{x}-\mathrm{y}}\end{array}$ & 10 & $\begin{array}{l}\text { Analyzing } \\
\text { Magr:et } \\
\text { Telescope }\end{array}$ & $\begin{array}{l}\text { Joint Institute for } \\
\text { Nuclear Research, } \\
\text { Synchrocyclotron }\end{array}$ & $\begin{array}{l}\text { L.S. Azhgirei et al., } \\
\text { Soviet Phys. - JETP } \\
939 \text { (1958) }\end{array}$ & . \\
\hline 94 & $\mathrm{p}$ & 0.670 & $\mathrm{C}$ & $\begin{array}{l}\text { Energy spectra and } \\
\text { angular distribution } \\
\text { of } \pi^{\circ}\end{array}$ & - & $\begin{array}{l}\text { Pair Gamma } \\
\text { Spectrometer }\end{array}$ & $\begin{array}{l}\text { Joint Institute for } \\
\text { Nuclear Research, } \\
\text { Synchrocyclotron }\end{array}$ & $\begin{array}{l}\text { IU.D. Baiukov, M.S. Kozodaev, } \\
\text { and A.A. Tiapkin, Soviet } \\
\text { Phys. JETP 5, } 552 \text { (1957) }\end{array}$ & $r$ \\
\hline 95 & $p$ & 0.730 & $\mathrm{C}$ & $\begin{array}{l}\text { Radio-chemical } \\
\text { cross sections }\end{array}$ & 8 & $\begin{array}{l}\text { Radio-Chemical } \\
\text { Techniques }\end{array}$ & $\begin{array}{l}\text { Berkeley, } \\
\text { Cyclotron }\end{array}$ & $\begin{array}{l}\text { M. Honda and D. Lal, } \\
\text { Phys. Rev. 118, } 1618 \\
\text { (1960) }\end{array}$ & \\
\hline 96 & $\mathrm{p}$ & 0.835 & $\mathrm{C}$ & $\begin{array}{l}\text { Radio-chemical } \\
\text { cross sections }\end{array}$ & $7-30$ & - & $\begin{array}{l}\text { Birmingham, } \\
\text { Synchrotron }\end{array}$ & $\begin{array}{l}\text { J.L. Symonds, J. Warren, } \\
\text { and J.D. Young, Proc. Phys. } \\
\text { Soc. A(London) } 70 \text {. } 824 \\
\text { (1957) }\end{array}$ & $\cdot$ \\
\hline
\end{tabular}




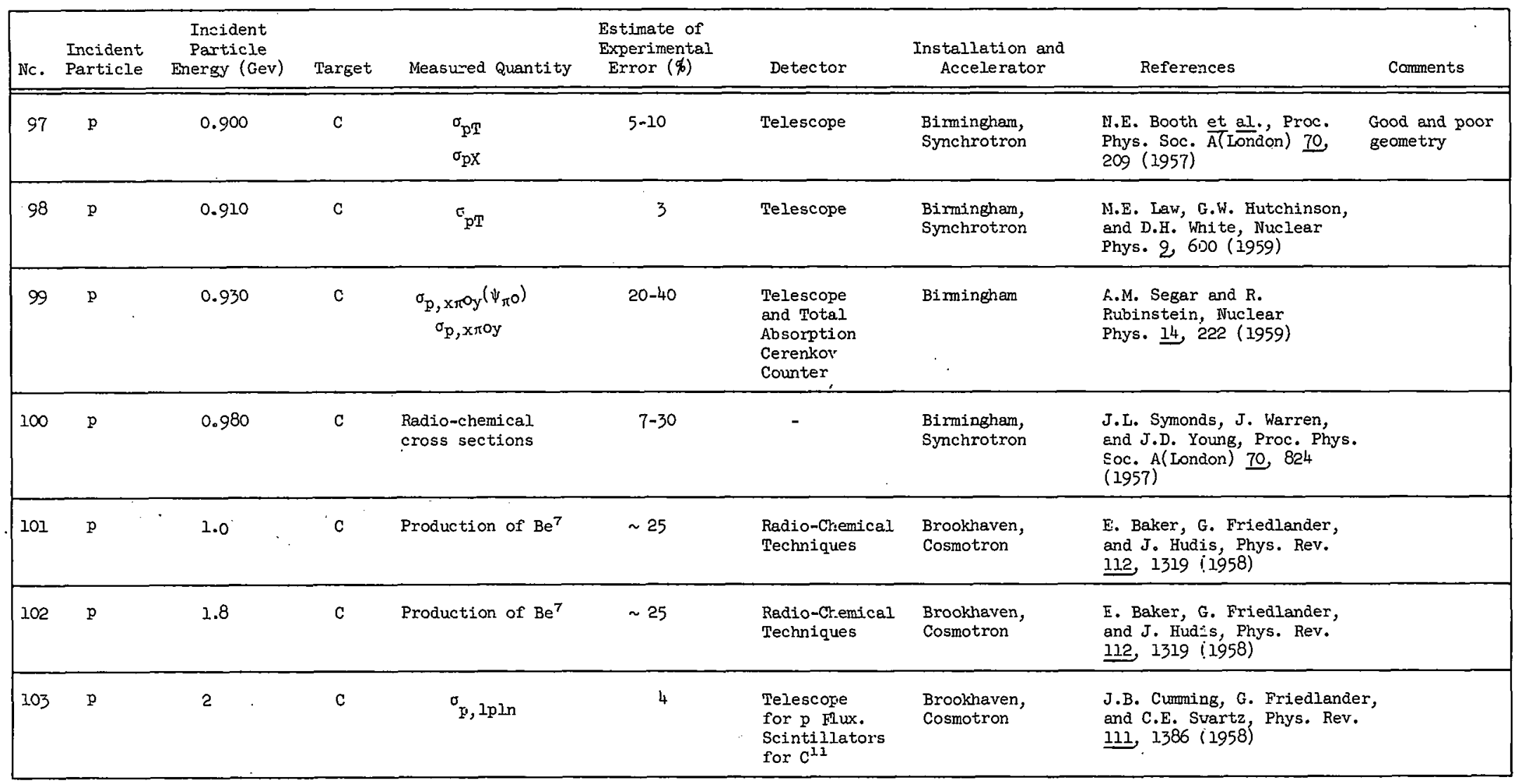




\begin{tabular}{|c|c|c|c|c|c|c|c|c|c|}
\hline No. & $\begin{array}{l}\text { Incident } \\
\text { Particle }\end{array}$ & $\begin{array}{c}\text { Incident } \\
\text { Particle } \\
\text { Energy (Gev) }\end{array}$ & Tarzet & Measured Quantity & $\begin{array}{l}\text { Estimate of } \\
\text { Experimental } \\
\text { Error }(\not)\end{array}$ & Detector . & $\begin{array}{c}\text { Installation and } \\
\text { Accelerator }\end{array}$ & References & Comments \\
\hline 104 & $\mathrm{p}$ & 2.2 & c & Production of $\mathrm{Be}^{7}$ & $\sim 25$ & $\begin{array}{l}\text { Radio-Chemical } \\
\text { Techniques }\end{array}$ & $\begin{array}{l}\text { Brookhaven, } \\
\text { Cosmotron }\end{array}$ & $\begin{array}{l}\text { E. Baker, G. Friedlander, } \\
\text { and J. Hudis, Phys. Rev. } \\
112 \text {, } 1319 \text { (1958) }\end{array}$ & \\
\hline 105 & $\mathrm{p}$ & 2.8 & c & $\sigma_{p X}$ & 5 & $\begin{array}{l}\text { Cloud } \\
\text { Chamber }\end{array}$ & $\begin{array}{l}\text { Brookhaven, } \\
\text { Cosmotron }\end{array}$ & $\begin{array}{l}\text { T. Bowen et al., Nuovo } \\
\text { cimento } 2 \text { 908 (1958) }\end{array}$ & \\
\hline 106 & $\mathrm{p}$ & 3.0 & $\mathrm{c}$ & $\sigma_{p, 1 p \ln }$ & 6 & $\begin{array}{l}\text { Plastic } \\
\text { Scintillators }\end{array}$ & $\begin{array}{l}\text { Berkeley, } \\
\text { Bevatron }\end{array}$ & $\begin{array}{l}\text { N. Horwitz and J.J. } \\
\text { Murray, Phys. Rev. } 117 \text {, } \\
1361 \text { (1960) }\end{array}$ & . \\
\hline 107 & $p$ & $3 \mathrm{Gev} / \mathrm{c}$ & $\mathrm{c}$ & $\begin{array}{l}\sigma_{\mathrm{p}, p} \\
\sigma_{\mathrm{pX}}\end{array}$ & $5-10$ & $\begin{array}{l}\text { Scintillation } \\
\text { Counters }\end{array}$ & $\begin{array}{l}\text { Berkeley, } \\
\text { Bevatron }\end{array}$ & $\begin{array}{l}\text { M.J. Longo and B.J. Moyer, } \\
\text { Phys. Rev. 125, } 701 \text {. } \\
\text { (1962) }\end{array}$ & $\begin{array}{l}\text { Good and poor } \\
\text { geometry }\end{array}$ \\
\hline 108 & $\mathrm{p}$ & 3.0 & $\mathrm{c}$ & Production of $\mathrm{Be}^{7}$ & $\sim 25$ & $\begin{array}{l}\text { Radio-Chemical } \\
\text { Techniques }\end{array}$ & $\begin{array}{l}\text { Brookhaven, } \\
\text { Cosmotron }\end{array}$ & $\begin{array}{l}\text { E. Baker, G. Friedlander, } \\
\text { and J. Hudis, Phys. Rev. } \\
112 \text { 1319 (1958) }\end{array}$ & \\
\hline 109 & $\mathrm{p}$ & 3 & $\mathrm{c}$ & $\sigma_{p, \operatorname{lpln}}$ & 4 & $\begin{array}{l}\text { Telescope } \\
\text { for p Flux. } \\
\text { Scintillators } \\
\text { for } \mathrm{c}^{11}\end{array}$ & $\begin{array}{l}\text { Brookhaven, } \\
\text { Cosmotron }\end{array}$ & $\begin{array}{l}\text { J.8. Cumming, G. Fried- } \\
\text { lander, and C.E. Swartz, } \\
\text { Phys. Rev. 111, } 1386 \\
(1958)\end{array}$ & \\
\hline 110 & $\mathrm{p}$ & $0.25-6.2$ & $\mathrm{c}^{12}$ & $\begin{array}{l}\text { Recoil ranges for } \\
\mathrm{C}^{11} \text {. Energy of } \\
\text { struck neutrons } \\
\text { inside } \mathrm{C}^{12} \text {. }\end{array}$ & 5 & $\begin{array}{l}\text { Plastíc } \\
\text { Target, Be } \\
\text { Catcher Foils }\end{array}$ & $\begin{array}{l}\text { Berkeley, } \\
\text { Cyclotron and } \\
\text { Bevatron }\end{array}$ & $\begin{array}{l}\text { Sarjant Singh and John } \\
\text { M. Alexander, Phys. Rev. } \\
\text { 128, } 711 \text { (1962) }\end{array}$ & . \\
\hline $11 i$ & $\mathrm{p}$ & 4.5 & c & $\sigma_{p, 1 p \ln }$ & 6 & $\begin{array}{l}\text { Plastic } \\
\text { Scintillators }\end{array}$ & $\begin{array}{l}\text { Berkeley, } \\
\text { Bevatron }\end{array}$ & $\begin{array}{l}\text { N. Horwitz and J.J. } \\
\text { Murray, Phys. Rev. } 117 \\
1361 \text { (1960) }\end{array}$ & \\
\hline
\end{tabular}




\begin{tabular}{|c|c|c|c|c|c|c|c|c|c|}
\hline No. & $\begin{array}{l}\text { Ircident } \\
\text { Particle }\end{array}$ & $\begin{array}{c}\text { Incident } \\
\text { Particle } \\
\text { Energy (Gev) }\end{array}$ & Target & Measured Quantity & $\begin{array}{l}\text { Estimate of } \\
\text { Experimental } \\
\text { Error }(\%)\end{array}$ & Detector & $\begin{array}{l}\text { Installation and } \\
\text { Accelerator }\end{array}$ & References & Conments \\
\hline 112 & $p$ & 5.7 & c & $\begin{array}{l}{ }^{\sigma} \mathrm{p}, \mathrm{lp} \text { In } \text { and other } \\
\text { radio-chemical cross } \\
\text { sections }\end{array}$ & 15 & NaI Crystal & $\begin{array}{l}\text { Berkeley, } \\
\text { Bevatron }\end{array}$ & $\begin{array}{l}\text { P.A. Benioff, Phys. Rev. } \\
\text { lig, } 316(1960)\end{array}$ & \\
\hline 113 & $\mathrm{p}$ & 6.0 & $\mathrm{c}$ & $\sigma_{p, 1 p l n}$ & 6 & $\begin{array}{l}\text { Plastic } \\
\text { Scintillators }\end{array}$ & $\begin{array}{l}\text { Berkeley, } \\
\text { Bevatron }\end{array}$ & $\begin{array}{l}\text { N. Horwitz and J.J. } \\
\text { M:array, Phys. Rev. 117, } \\
1361 \text { (1960) }\end{array}$ & \\
\hline 114 & p & Energy: $>7$ & $\mathrm{c}$ & $\sigma_{p X}$ & 15 & $\begin{array}{l}\text { Hodoscopic } \\
\text { Counters }\end{array}$ & $\begin{array}{l}\text { Moscow University, } \\
\text { Stratosphere }\end{array}$ & $\begin{array}{l}\text { K.I. Alekseeva and N.L. } \\
\text { Grigorov, Soviet Phys.- } \\
\text { J } 3 \text { TP } 535 \text { (1957) }\end{array}$ & . \\
\hline 115 & $\mathrm{p}$ & Energy $>7$ & $\mathrm{c}$ & $\begin{array}{l}\sigma_{p} \mathrm{X} \text {; average number } \\
\text { of charged particles }\end{array}$ & 15 & Telescope & $\begin{array}{l}\text { Moscow, } \\
\text { Stratosphere }\end{array}$ & $\begin{array}{l}\text { K.I. Alekseeva and N.L. } \\
\text { Grigorov, Soviet Phys. - } \\
\text { JETP } 8,416 \text { (1959) }\end{array}$ & \\
\hline 116 & p & $0.4-17$ & $\mathrm{C}^{12}$ & $\begin{array}{l}\text { Ratio of } \mathrm{Al}^{27}(\mathrm{p}, 3 \mathrm{pn}) \\
\mathrm{Na}^{24} / \mathrm{C}^{12}(\mathrm{p}, \mathrm{pn}) \mathrm{C}^{11^{2}} \\
\text { cross sections }\end{array}$ & 5 & $\begin{array}{l}\text { Scintillation } \\
\text { and Propor- } \\
\text { tional Counters }\end{array}$ & $\begin{array}{l}\text { Brookhaven, } \\
\text { Symchrotron }\end{array}$ & 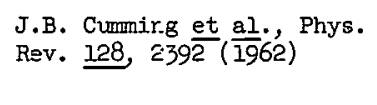 & \\
\hline 117 & $\mathrm{p}$ & $\sim 12$ & $\mathrm{c}$ & $\sigma_{p x}$ & 15 & $\begin{array}{l}\text { Telescope } \\
\text { Hodoscopic } \\
\text { Counters }\end{array}$ & $\begin{array}{l}\text { Moscow University, } \\
\text { Stratosphere }\end{array}$ & $\begin{array}{l}\text { K.I. Alekseeva and N.L. } \\
\text { Grigorov, Soviet Phys. } \\
\text { Doklady 2, } 317 \text { (1957) }\end{array}$ & \\
\hline 118 & $p$ & $15.9 \mathrm{Gev} / \mathrm{c}$ & $\mathrm{c}$ & $\begin{array}{l}\text { Proton momentum } \\
\text { spectrum at } \sim 60 \\
\text { mrad }\end{array}$ & 50 & - & $\begin{array}{c}\text { CERN, } \\
\text { Synchrotron }\end{array}$ & $\begin{array}{l}\text { G. Cocconi et al., Phys. } \\
\text { Rev. } 126,277 \text { (1962) }\end{array}$ & $n$ \\
\hline 219 & $\mathrm{p}$ & $18.3 \mathrm{Gev} / \mathrm{c}$ & $\mathrm{c}$ & $\begin{array}{l}\text { Proton momentum } \\
\text { spectrum at } \sim 60 \\
\text { mrad }\end{array}$ & 50 & - & $\begin{array}{c}\text { CERN, } \\
\text { Synchrotron }\end{array}$ & $\begin{array}{l}\text { G. Cocconi et al., Phys. } \\
\text { Rev. } 126,277 \frac{\text { al }}{(1962)}\end{array}$ & $\begin{array}{l}\because \\
\because \cdots \\
\therefore\end{array}$ \\
\hline
\end{tabular}




\begin{tabular}{|c|c|c|c|c|c|c|c|c|c|}
\hline No. & $\begin{array}{l}\text { Incident } \\
\text { Particle }\end{array}$ & $\begin{array}{c}\text { Incident } \\
\text { Particle } \\
\text { Energy (Gev) }\end{array}$ & Target & Measured Quantity & $\begin{array}{l}\text { Estimate of } \\
\text { Experimental } \\
\text { Error }(\phi)\end{array}$ & Detector & $\begin{array}{l}\text { Installation and } \\
\text { Accelerator }\end{array}$ & References & Comments \\
\hline 120 & $\mathrm{p}$ & 24 & C & $\begin{array}{l}\text { Transverse momentum } \\
\text { spectrum of } \pi^{\circ}\end{array}$ & 8 & $\begin{array}{l}\text { Emulsion } \\
\text { Stack }\end{array}$ & $\begin{array}{l}\text { Bombay; Exposure } \\
\text { Made at CERN } \\
\text { Synchrotron }\end{array}$ & $\begin{array}{l}\text { Y. Pal and T. } \\
\text { Rengarajan, Phys. Rev. } \\
124,1575 \text { (1961) }\end{array}$ & \\
\hline 121 & $\mathrm{p}$ & 24 & c & $\begin{array}{l}\text { Average energy; most } \\
\text { probable and average } \\
\text { traiśngerse momentum } \\
\text { of } \pi^{\circ}\end{array}$ & $5-20$ & $\begin{array}{l}\text { Emulsion } \\
\text { Stack }\end{array}$ & $\begin{array}{l}\text { Tata Institute, } \\
\text { India; Exposure } \\
\text { Made at CERN } \\
\text { Synchrotron }\end{array}$ & $\begin{array}{l}\text { Y. Pal and T. Rengarajan, } \\
\text { J. Phys. Soc. Japan } 17 \\
\text { sup. A-III, } 387(1962)\end{array}$ & . \\
\hline 122 & $\mathrm{p}$ & $26.0 \mathrm{Gev} / \mathrm{c}$ & $\mathrm{c}$ & $\begin{array}{l}\text { Proton momentum } \\
\text { spectrum at } \sim 60 \\
\text { mrad }\end{array}$ & 50. & - & $\begin{array}{l}\text { CERN, } \\
\text { Synchrotron }\end{array}$ & $\begin{array}{l}\text { G. Cocconi et al., Phys. } \\
\text { Rev. 126, } 277 \text { (1962) }\end{array}$ & \\
\hline 123 & $\mathrm{p}$ & 28 & $\mathrm{C}^{12}$ & $\sigma_{p, l p l n}$ & 5 & $\begin{array}{l}\text { Plastic } \\
\text { Scintillators }\end{array}$ & $\begin{array}{l}\text { Brookhaven, } \\
\text { Synchrotron }\end{array}$ & $\begin{array}{l}\text { J.B. Cumming, G. Fried- } \\
\text { lander, and S. Katcoff, } \\
\text { Phys. Rev. 125, } 2078 \text { (1962) }\end{array}$ & \\
\hline 124 & $\mathrm{p}$ & 0.096 & $\mathrm{~N}$ & $\sigma_{p, x p y}\left(E_{p}, 40^{\circ}\right)$ & 5 & $\begin{array}{l}\text { Range } \\
\text { Telescope }\end{array}$ & $\begin{array}{l}\text { Harvard, } \\
\text { Cyclotron }\end{array}$ & $\begin{array}{l}\text { K. Strauch and F. Titus, } \\
\text { Phys. Rev. 104, } 191 \text { (1956) }\end{array}$ & \\
\hline 125 & $\mathrm{p}$ & 0.142 & $\mathrm{~N}$ & $\begin{array}{l}\sigma_{\mathrm{p} T} \\
\sigma_{\mathrm{p}, \mathrm{p}}\end{array}$ & $2-10$ & $\begin{array}{l}\text { Counter } \\
\text { Telescope }\end{array}$ & $\begin{array}{l}\text { Harwell, } \\
\text { Synchrocyclotron }\end{array}$ & $\begin{array}{l}\text { A.E. Taylor and E. Wood, } \\
\text { Nuclear Phys. } 25,642 \\
\text { (1961) }\end{array}$ & Good geometry \\
\hline 126 & $\mathrm{p}$ & 0.185 & $\mathrm{~N}$ & $\begin{array}{l}\sigma_{p, x p y}\left(E_{p}, \psi_{p}\right) \text { with } \\
\psi_{p}=5^{\circ}, 7^{\circ}, 10^{\circ}, 18^{\circ}\end{array}$ & $15-40$ & - & Upsala & $\begin{array}{l}\text { H. Tyrén and Th.A.J. Maris, } \\
\text { Nuclear Phys. } 6 \text {, } 82 \text { (1958) }\end{array}$ & \\
\hline 127 & $\mathrm{p}$ & 0.4 & $\mathrm{~N}^{14}$ & $\sigma_{p, l p l n}$ & $\sim 20$ & $\begin{array}{l}\text { Radio-Chenical } \\
\text { Techniques }\end{array}$ & $\begin{array}{l}\text { Brookhaven, } \\
\text { Cosmotron; } \\
\text { Nevis Synchrocy- } \\
\text { clotron, Columbia }\end{array}$ & $\begin{array}{l}\text { S.S. Markowitz, F.S. Row- } \\
\text { land, G. Friedlander, Phys. } \\
\text { Rev. 112, } 1295 \text { (1958) }\end{array}$ & \\
\hline
\end{tabular}




\begin{tabular}{|c|c|c|c|c|c|c|c|c|c|}
\hline No. & $\begin{array}{l}\text { Inciden= } \\
\text { Particle }\end{array}$ & $\begin{array}{c}\text { Incident } \\
\text { Particle } \\
\text { Energy (Gev) }\end{array}$ & Target & Measwred Quantity & $\begin{array}{l}\text { Estimate of } \\
\text { Experimental } \\
\text { Error }(\phi)\end{array}$ & Detector & $\begin{array}{l}\text { Installation and } \\
\text { Accelerator }\end{array}$ & References & Comments \\
\hline 128 & $p$ & 0.420 & $\mathrm{~N}$ & $\begin{array}{l}\text { Radio-chemical } \\
\text { cross sections }\end{array}$ & $7-30$ & $\cdots \quad \cdots$ & $\begin{array}{l}\text { Birmingham, } \\
\text { Synchrotron }\end{array}$ & $\begin{array}{l}\text { J.L. Symonds, J. Warren, } \\
\text { and J.D. Young, Proc. } \\
\text { Phys. Soc. A(London) 70, } \\
\text { 324 (1957) }\end{array}$ & \\
\hline 129 & $\mathrm{p}$ & 0.522 & $\mathrm{~N}$ & $\begin{array}{l}\text { Radio-chemical } \\
\text { cross sections }\end{array}$ & $7-30$ & - & $\begin{array}{l}\text { Birmingham, } \\
\text { Synchrotron }\end{array}$ & $\begin{array}{l}\text { J.L. Symor.ds, J. Warre n, } \\
\text { and.J.D. Young, Proc. } \\
\text { Phys. Soc. A(London) } \\
70,824 \text { (1957) }\end{array}$ & \\
\hline 130 & $p$ & 0.648 & $\mathrm{~N}$ & $\begin{array}{l}\text { Radio-shemical } \\
\text { cross sections }\end{array}$ & $7-30$ & - & $\begin{array}{l}\text { Birmingham, } \\
\text { Synchrotron }\end{array}$ & $\begin{array}{l}\text { J.L. Symonds, J. Warren, } \\
\text { and J.D. Young, Proc. } \\
\text { Phys, Soc. A(London) } \\
70,824 \text { (1957) }\end{array}$ & \\
\hline 131 & $\mathrm{p}$ & 0.660 & $\mathbb{N}$ & $\begin{array}{l}\text { Black track number } \\
\text { distrijution. Proton } \\
\text { and aljha energy dis- } \\
\text { tribution and angular } \\
\text { distrioution }\end{array}$ & $\sim 10-25$ & $\begin{array}{l}\text { Sandwicin } \\
\text { Emulsion }\end{array}$ & $\begin{array}{l}\text { Radium Institute; Ex- } \\
\text { posure Made at Joint } \\
\text { Institute for Nuclear } \\
\text { Research } \\
\text { jynchrocyclotron }\end{array}$ & $\begin{array}{l}\text { N.A. Perfilov and Yu.I. } \\
\text { Serebrennikov, Soviet } \\
\text { Phys.-JETP 13, } 274 \\
\text { (1961). }\end{array}$ & \\
\hline 132 & $\mathrm{p}$ & 0.835 & $\mathbb{N}$ & $\begin{array}{l}\text { Radio-chemical } \\
\text { cross sections }\end{array}$ & $7-30$ & - & $\begin{array}{l}\text { Birmingham, } \\
\text { Synchrotron }\end{array}$ & $\begin{array}{l}\text { J.L. Symonja, J. Warren, } \\
\text { and J.D. Young, Proc. Phys. } \\
\text { Soc. A(London) 0, } 824 \\
\text { (1957) }\end{array}$ & \\
\hline 133 & $\mathrm{p}$ & 0.980 & N & $\begin{array}{l}\text { Radio-chemical } \\
\text { cross sections }\end{array}$ & $7-30$ & - & $\begin{array}{l}\text { Birmingham, } \\
\text { Symchrotron }\end{array}$ & $\begin{array}{l}\text { J.L. Symonds, J. Warren, } \\
\text { end J.D. Young, Proc. } \\
\text { Phys. Soc. A(London) } \\
\text { IO } 824 \text { (1957) }\end{array}$ & \\
\hline 134 & $\mathrm{p}$ & 3 & $\mathrm{~N}^{14}$ & $\sigma_{p, \text { Ipln }}$ & $\sim 20$ & $\begin{array}{l}\text { Radio-Chemical } \\
\text { Techniques }\end{array}$ & $\begin{array}{l}\text { Brookhaven, } \\
\text { Cosmotron; } \\
\text { Ilevis Synchrocy- } \\
\text { clotron, Columbia }\end{array}$ & $\begin{array}{l}\text { S.S. Markoritz, F.S. } \\
\text { P.owland, and G. Friedlander, } \\
\text { Phys. Rev. 112, } 1295 \text { (1958) }\end{array}$ & \\
\hline
\end{tabular}




\begin{tabular}{|c|c|c|c|c|c|c|c|c|c|}
\hline No. & $\begin{array}{l}\text { Incident } \\
\text { Particle }\end{array}$ & $\begin{array}{c}\text { Incident } \\
\text { Particle } \\
\text { Energy (Gev) }\end{array}$ & Target & Measured Quantity & $\begin{array}{l}\text { Estimate of } \\
\text { Experimental } \\
\text { Error }(\%)\end{array}$ & Detector & $\begin{array}{l}\text { Installation and } \\
\text { Accelerator }\end{array}$ & References & Comments \\
\hline 135 & $\mathrm{p}$ & 5.7 & $\mathrm{~N}$ & $\begin{array}{l}\sigma_{p, l p l n} \text { and other } \\
\text { radio-chernical } \\
\text { cross sections }\end{array}$ & 15 & NaI Crystal & $\begin{array}{l}\text { Berkeley, } \\
\text { Bevatron }\end{array}$ & $\begin{array}{l}\text { P.A. Benioff, Phys. } \\
\text { Rev. 119, } 316(1960) \text {. }\end{array}$ & \\
\hline 136 & $\mathrm{p}$ & 0.096 & 0 & $\sigma_{p, x p y}\left(E_{p}, 40^{\circ}\right)$ & 5 & $\begin{array}{l}\text { Range } \\
\text { Telescope }\end{array}$ & $\begin{array}{l}\text { Harvard, } \\
\text { Cyclotron }\end{array}$ & $\begin{array}{l}\text { K. Strauch and F. Titus, } \\
\text { Phys. Rev. 104, } 191 \\
\text { (1956) }\end{array}$ & . \\
\hline 137 & $\mathrm{p}$ & 0.142 & 0 & $\begin{array}{l}\sigma_{p T} \\
\sigma_{p, p}\end{array}$ & $2-10$ & $\begin{array}{l}\text { Counter } \\
\text { Telescope }\end{array}$ & $\begin{array}{l}\text { Harwell, } \\
\text { Synchrocyclotron }\end{array}$ & $\begin{array}{l}\text { A.E. Taylor and E. Wood, } \\
\text { Nuclear Phys. } 25,642 \\
\text { (1961) }\end{array}$ & Good geometry \\
\hline 138 & $\mathrm{p}$ & 0.155 & 0 & $\begin{array}{l}\mathrm{Li}^{6} \text { and } \mathrm{Be}^{7} \text { produc- } \\
\text { tion cross } \\
\text { sections }\end{array}$ & 30 & $\begin{array}{l}\text { Isotope } \\
\text { Dilution } \\
\text { Method }\end{array}$ & $\begin{array}{c}\text { Orsay, } \\
\text { Synchrocyclotron }\end{array}$ & $\begin{array}{l}\text { E. Gradsztajn, J. phys. } \\
\text { radium 21, } 761 \text { (1960) }\end{array}$ & \\
\hline 139 & $\mathrm{p}$ & 0.185 & 0 & $\begin{array}{l}\text { Nuclear shell struc- } \\
\text { ture from } \\
\sigma_{p, 2 p}\left(\mathrm{E}_{\mathrm{pl}}, \mathrm{E}_{\mathrm{p} 2}, \psi_{\mathrm{pl}}, \psi_{\mathrm{p} 2}\right)\end{array}$ & 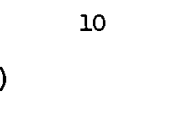 & $\begin{array}{l}\text { Double Tele- } \\
\text { scope Range } \\
\text { Method for } \\
\text { Energy }\end{array}$ & Upsala & $\begin{array}{l}\text { H. Tyrén, P. Hillman, and } \\
\text { Th.A.J. Maris, Nuclear } \\
\text { Phys. } \mathcal{I} \text { 10 (1958) }\end{array}$ & \\
\hline 140 & $p$ & 0.222 & 0 & $\begin{array}{l}\text { Radio-chemical } \\
\text { cross sections }\end{array}$ & $3-40$ & Counters & $\begin{array}{l}\text { Liverpool, } \\
\text { Synchrocyclotron }\end{array}$ & $\begin{array}{l}\text { V Parikh, Nuclear Phys. } \\
18,646(1960)\end{array}$ & \\
\hline 141 & $\mathrm{p}$ & 0.279 & 0 & $\begin{array}{l}\text { Radio-chemical } \\
\text { cross sections }\end{array}$ & $3-40$ & Counters & $\begin{array}{l}\text { Liverpool, } \\
\text { Synchrocyclotron }\end{array}$ & $\begin{array}{l}\text { V. Parikh, Nuclear Phys. } \\
18,646(1960)\end{array}$ & \\
\hline 142 & $\mathrm{p}$ & $0.100-0.500$ & 0 & $\begin{array}{l}\text { Distribution of fast } \\
\text { prongs. Angular dis- } \\
\text { tribution between } 2 \\
\text { secondaries }\end{array}$ & $5-40$ & $\begin{array}{l}\text { Cloud } \\
\text { Chamber }\end{array}$ & $\begin{array}{c}\text { Milan } \\
\text { (Cosmic Rays) }\end{array}$ & $\begin{array}{l}\text { E. F1orini and s. Ratti, } \\
\text { Nuovo cimento 14, } 901 \\
\text { (1959) }\end{array}$ & \\
\hline
\end{tabular}




\begin{tabular}{|c|c|c|c|c|c|c|c|c|c|}
\hline Nc. & $\begin{array}{l}\text { Incident } \\
\text { Particle }\end{array}$ & $\begin{array}{c}\text { Incident } \\
\text { Particle } \\
\text { Energy (Gev) }\end{array}$ & Target & Measured Quantity & $\begin{array}{l}\text { Estimate of } \\
\text { Experimental } \\
\text { Error }(\phi)\end{array}$ & Detector & $\begin{array}{l}\text { Installation and } \\
\text { Accelerator }\end{array}$ & References . & Comments \\
\hline 143 & $p$ & 0.314 & 0 & $\begin{array}{l}\text { Radio-chemical } \\
\text { cross sections }\end{array}$ & $3-40$ & Counters & $\begin{array}{l}\text { Liverpool, } \\
\text { Synchrocyclotron }\end{array}$ & $\begin{array}{l}\text { Ir. Parikh, Nuclear Phys. } \\
18,646(1960)\end{array}$ & \\
\hline 144 & $\mathrm{p}$ & 0.362 & 0 & $\begin{array}{l}\text { Radio-chemical } \\
\text { cross sections }\end{array}$ & $3-40$ & Counters & $\begin{array}{l}\text { Liverpool, } \\
\text { Synchrocyclotron }\end{array}$ & $\begin{array}{l}\text { v. Parikh, Nuclear Phys. } \\
\text { 18 } 646(1960)\end{array}$ & \\
\hline 245 & $\mathrm{p}$ & 0.420 & 0 & $\begin{array}{l}\text { Radio-chemical } \\
\text { cross sections }\end{array}$ & $7-30$ & $-\cdot$ & $\begin{array}{l}\text { Birmingham, } \\
\text { Synchrotron }\end{array}$ & $\begin{array}{l}\text { J.L. Symonds, J. Warren, } \\
\text { End J.D. Young, Proc. Phys. } \\
\text { Soc. A(London) } 70,824 \text { ( } 1957\end{array}$ & \\
\hline 145 & $\mathrm{p}$ & 0.522 & 0 & $\begin{array}{l}\text { Radio-chemical } \\
\text { cross sections }\end{array}$ & $7-30$ & - & $\begin{array}{l}\text { Birmingham, } \\
\text { Synchrotron }\end{array}$ & $\begin{array}{l}\text { J.L. Symonds, J. Warren, } \\
\text { and J.D. Young, Proc. Phys. } \\
\text { Soc. A(Iondon) ㅁ } 824 \text { (1957 }\end{array}$ & \\
\hline 147 & $\mathrm{p}$ & 0.648 & 0 & $\begin{array}{l}\text { Radio-chemical } \\
\text { cross sections }\end{array}$ & $7-30$ & - & $\begin{array}{l}\text { Birmingham, } \\
\text { Synchrotron }\end{array}$ & $\begin{array}{l}\text { J.L. Symonds, J. Warren, } \\
\text { and J.D. Young, Proc. Phys. } \\
\text { Soc. A(Iondon) 70 } 824 \text { ( } 1957\end{array}$ & \\
\hline 149 & $p$ & 0.660 & $\therefore 0$ & $\begin{array}{l}\text { Black track number } \\
\text { distrikution. Proton } \\
\text { and alfha energy dis- } \\
\text { tribution and angular } \\
\text { distrikution }\end{array}$ & $\sim 10-25$ & $\begin{array}{l}\text { Sandwich } \\
\text { Emulsions }\end{array}$ & $\begin{array}{l}\text { Fadium Institute; } \\
\text { Fxposure Made at } \\
\text { Joint Institute for } \\
\text { Nuclear Research } \\
\text { Synchrocyclotron }\end{array}$ & $\begin{array}{l}\text { N.A. Perfiiov and Yu.I. } \\
\text { Serebrennit:ov, Soviet } \\
\text { Phys. JEIP 13, } 274 \\
\text { (196i) }\end{array}$ & . \\
\hline 150 & $\mathrm{p}$ & 0.835 & 0 & $\begin{array}{l}\text { Radio-chemical } \\
\text { cross sections }\end{array}$ & $7-30$ & - & $\begin{array}{l}\text { Birmingham, } \\
\text { Synchrotron }\end{array}$ & $\begin{array}{l}\text { J.L. Symonàs, J. Warren, } \\
\text { and J.D. Young, Proc. Phys. } \\
\text { Soc. A(Lonãon) 70, } 824 \\
(1957)\end{array}$ & \\
\hline
\end{tabular}




\begin{tabular}{|c|c|c|c|c|c|c|c|c|}
\hline No. & $\begin{array}{l}\text { Incident } \\
\text { Particle }\end{array}$ & $\begin{array}{c}\text { Incident } \\
\text { Particle } \\
\text { Energy (Gev) }\end{array}$ & Target & Measured quantity & $\begin{array}{l}\text { Estimate of } \\
\text { Experimental } \\
\text { Error }(\%)\end{array}$ & Detector & $\begin{array}{l}\text { Installation and } \\
\text { Accelerator }\end{array}$ & References \\
\hline 151 & $\mathrm{p}$ & 0.910 & 0 & $\sigma_{\mathrm{pT}}$ & 3 & Telescope. & $\begin{array}{l}\text { Birmingham, } \\
\text { Symchrotron }\end{array}$ & $\begin{array}{l}\text { M.E. Law, G.W. Hutchinson, } \\
\text { and D.H. White, Nuclear } \\
\text { Phys. 2, } 600 \text { (1959) }\end{array}$ \\
\hline 152 & $p$ & 0.980 & 0 & $\begin{array}{l}\text { Radio-chemical } \\
\text { cross sections }\end{array}$ & $7-30$ & - & $\begin{array}{l}\text { Birmingham, } \\
\text { Synchrotron }\end{array}$ & $\begin{array}{l}\text { J.I. Symonds, J. Warren, } \\
\text { and J.D. Young, Proc. Phys. } \\
\text { Soc. A(London) } 70 \text {, } 824 \text { (1957) }\end{array}$ \\
\hline 153 & $\mathrm{p}$ & 5.7 & 0 & $\begin{array}{l}\sigma_{\mathrm{p}} \text {, lpln and other } \\
\text { radio-ciemical } \\
\text { cross sections }\end{array}$ & 15 & NaI Crovstal & $\begin{array}{l}\text { Berkeley, } \\
\text { Bevatron }\end{array}$ & $\begin{array}{l}\text { P.A. Benioff, Phys. Rev. } \\
\text { 119 } 316(1960)\end{array}$ \\
\hline 154 & $\mathrm{p}$ & $0.020-0.150$ & $\mathrm{~F}$ & ${ }^{\sigma} p$, lpln & 30 & $\begin{array}{l}\text { Radio-Chemical } \\
\text { Techniques }\end{array}$ & $\begin{array}{c}\text { Orsay, } \\
\text { Synchrocyclotron }\end{array}$ & $\begin{array}{l}\text { M. Gusakow, Ann. phys. } \\
\text { (Paris) I, } 67 \text { (1962) }\end{array}$ \\
\hline 255 & $\mathrm{p}$ & $0.020-0.155$ & $\mathrm{~F}$ & $\sigma_{p, \operatorname{lp} \ln }$ & $\sim 30$ & $\begin{array}{l}\text { Radio-Chemical } \\
\text { Technique }\end{array}$ & $\begin{array}{c}\text { Orsay, } \\
\text { Synchrocyclotron }\end{array}$ & $\begin{array}{l}\text { M. Gusakow et al., J. phys. } \\
\text { radium 22, } 636 \text { (1961) }\end{array}$ \\
\hline 156 & $p$ & 0.096 & F & $\sigma_{p, x F y}\left(E_{p}, 40^{\circ}\right)$ & 5 & $\begin{array}{l}\text { Range } \\
\text { Telescope }\end{array}$ & $\begin{array}{l}\text { Harvard, } \\
\text { Cyclotron }\end{array}$ & $\begin{array}{l}\text { K. Strauch and F. Titus, } \\
\text { Phys. Rev. 104 } 191 \text { (1956) }\end{array}$ \\
\hline 157 & $p$ & 0.185 & F & $\begin{array}{l}\text { Nuclear level struc- } \\
\text { ture from proton } \\
\text { spectrum }\end{array}$ & - & Telescope & Upsala & $\begin{array}{l}\text { H. Tyren and Th.A.J. Maris, } \\
\text { Nuclear Phys. } 6,446 \text { (1958) }\end{array}$ \\
\hline i58 & $p$ & 0.222 & $\mathrm{~F}$ & $\begin{array}{l}\text { Radio-chemical } \\
\text { cross sections }\end{array}$ & $3-40$ & Counters & $\begin{array}{l}\text { Liverpool, } \\
\text { Synchrocyclotron }\end{array}$ & $\begin{array}{l}\text { V. Parikh, Nuclear Phys. } \\
18,646(1960)\end{array}$ \\
\hline 159 & $p$ & $0.082-0.426$ & $F^{19}$ & $\sigma_{p, I p l n}$ & $2-10$ & $\begin{array}{l}\text { Radio-Themical } \\
\text { Techniques }\end{array}$ & $\begin{array}{l}\text { Chicago, } \\
\text { Synchrocyclotron }\end{array}$ & $\begin{array}{l}\text { H.P. Yule and A. Turkevich, } \\
\text { Phys. Rev. 118, } 1591 \text { (1960) }\end{array}$ \\
\hline
\end{tabular}




\begin{tabular}{|c|c|c|c|c|c|c|c|c|}
\hline No. & $\begin{array}{l}\text { Incident } \\
\text { Particle }\end{array}$ & $\begin{array}{c}\text { Trcident } \\
\text { Particle } \\
\text { Energy (Gev) }\end{array}$ & Target & Measured Quantity & $\begin{array}{l}\text { Estimate of } \\
\text { Experimental } \\
\text { Error }(\%)\end{array}$ & Detector & $\begin{array}{l}\text { Installation and } \\
\text { Accelerator }\end{array}$ & References \\
\hline 160 & $\mathrm{p}$ & c. 279 & $\mathrm{~F}$ & $\begin{array}{l}\text { Radio-chemical } \\
\text { cross sections }\end{array}$ & $3-40$ & Counters & $\begin{array}{l}\text { Liverpool, } \\
\text { Symchrocyclotron }\end{array}$ & $\begin{array}{l}\text { y. Parikh, Nuclear Phys. } \\
18,646(1960)\end{array}$ \\
\hline 161 & $p$ & 0.314 & $\mathrm{~F}$ & $\begin{array}{l}\text { Radio-chemical } \\
\text { cross sections }\end{array}$ & $3-40$ & Counters & $\begin{array}{l}\text { Liverpool, } \\
\text { Synchrocyclotron }\end{array}$ & $\begin{array}{l}\text { v. Parikh, Nuclear Phys. } \\
=8,646(1960)\end{array}$ \\
\hline 162 & $\mathrm{p}$ & 0.362 & $\mathrm{~F}$ & $\begin{array}{l}\text { Radio-chemical } \\
\text { cross sections }\end{array}$ & $3-40$ & Counters & $\begin{array}{l}\text { Liverpool, } \\
\text { Synchrocyclotron }\end{array}$ & $\begin{array}{l}\text { T. Parikh, Nuclear Phys. } \\
18,646(1960)\end{array}$ \\
\hline 163 & $\mathrm{p}$ & $0.4^{\circ}$ & $F^{1 \subseteq}$ & ${ }^{\circ} \mathrm{p}, \operatorname{lp} \ln$ & $\sim 20$ & $\begin{array}{l}\text { Radio-Chemical } \\
\text { Techniques }\end{array}$ & $\begin{array}{l}\text { Brookhaven, } \\
\text { Cosmotron; Nevis } \\
\text { Synchrocyclotron, } \\
\text { Columbia }\end{array}$ & $\begin{array}{l}\text { S.S. Markowitz, F.S. Rowland, } \\
\text { End G. Friedlander, Phys. } \\
\text { Rev. 112, } 1295 \text { (1958) }\end{array}$ \\
\hline 164 & $\mathrm{p}$ & 0.420 & $\mathrm{~F}$ & $\begin{array}{l}\text { Radio-chemical } \\
\text { cross sections }\end{array}$ & $7-30$ & - & $\begin{array}{l}\text { Birminghar, } \\
\text { Synchrotron }\end{array}$ & $\begin{array}{l}\text { J.L. Symonds, J. Warren, } \\
\text { and J.D. Young, Proc. } \\
\text { Fhys Soc. A(Iondon) 70, } \\
\text { E24 (1957) }\end{array}$ \\
\hline 165 & $\mathrm{p}$ & 0.522 & F & $\begin{array}{l}\text { Radio-chemical } \\
\text { cross sections }\end{array}$ & $7-30$ & - & $\begin{array}{l}\text { Birmingham, } \\
\text { Synchrotron }\end{array}$ & $\begin{array}{l}\text { J.L. Symonds, J. Warren, } \\
\text { and J.D. Young, Proc. Phys. } \\
\text { Soc. A(London) T0, } 824 \\
\text { (1957) }\end{array}$ \\
\hline 166 & $\mathrm{p}$ & 0.648 & F & $\begin{array}{l}\text { Radio-chemical } \\
\text { cross sections }\end{array}$ & $7-30$ & - & $\begin{array}{l}\text { Birmingham, } \\
\text { Synchrotron }\end{array}$ & $\begin{array}{l}\text { J.L. Symoncs, J. Warren, } \\
\text { and J.D. Ycung, Proc. } \\
\text { Pays. Soc. A(Iondon) 70, } \\
824 \text { (1957) }\end{array}$ \\
\hline 167 & $p$ & 0.835 & $\mathrm{~F}$ & $\begin{array}{l}\text { Radio-chemical } \\
\text { cross sections }\end{array}$ & $7-30$ & - & $\begin{array}{l}\text { Birmingham, } \\
\text { Synchrotron }\end{array}$ & $\begin{array}{l}\text { J.L. Symonds, J. Warren, } \\
\text { and J.D. Ycung, Proc. } \\
\text { Phys. Soc. A(London) 70, } \\
824 \text { (1957) }\end{array}$ \\
\hline
\end{tabular}




\begin{tabular}{|c|c|c|c|c|c|c|c|c|}
\hline No. & $\begin{array}{l}\text { Incident } \\
\text { Particle }\end{array}$ & $\begin{array}{c}\text { Incident } \\
\text { Part_cle } \\
\text { Inergy (Gev) }\end{array}$ & Target & Measured Quantity & $\begin{array}{l}\text { Estimate of } \\
\text { Experimental } \\
\text { Error }(\%)\end{array}$ & Detector & $\begin{array}{l}\text { Installation and } \\
\text { Accelerator }\end{array}$ & References \\
\hline 168 & $\mathrm{p}$ & 0.980 & F & $\begin{array}{l}\text { Radio-chemical } \\
\text { cross sections }\end{array}$ & $7-30$ & - & $\begin{array}{l}\text { Binninghem, } \\
\text { Synchrotron }\end{array}$ & $\begin{array}{l}\text { J.L. Symonds, J. Warren, } \\
\text { and J.D. Young, Proc. } \\
\text { Phys. Soc. A(London) } 70 \\
824 \text { (1957) }\end{array}$ \\
\hline 169 & $\underline{p}$ & $0.28-2.2$ & $F^{19}$ & $\sigma_{p, \operatorname{lp} \ln }$ & $\sim 20$ & $\begin{array}{l}\text { Radio-Chenical } \\
\text { Techniques }\end{array}$ & $\begin{array}{l}\text { Brookhaven, } \\
\text { Cosmotron; Nevis } \\
\text { Synchrocyclotron, } \\
\text { Columbia }\end{array}$ & $\begin{array}{l}\text { S.S. Markowitz, F.S. Rowland, } \\
\text { and G. Friedlander, Phys. Rev. } \\
\text { 112, } 1295 \text { (1958) }\end{array}$ \\
\hline 170 & 9 & 3 & $F^{19}$ & $\sigma_{p, 2 p \ln }$ & $\sim 20$ & $\begin{array}{l}\text { Radio-Chemical } \\
\text { Techniques }\end{array}$ & $\begin{array}{l}\text { Brookhaven, } \\
\text { Cosmotron; ivevis } \\
\text { Synchrocyclotron, } \\
\text { Columbia }\end{array}$ & $\begin{array}{l}\text { S.S. Markowitz, F.s. Rowland, } \\
\text { and G. Friedlander, Phys. } \\
\text { Rev. I12 } 1295 \text { (1958) }\end{array}$ \\
\hline 171 & $\mathrm{p}$ & 5.7 & $F$ & $\begin{array}{l}\sigma_{\mathrm{p}, l p l n} \text { and other } \\
\text { radio-chemical } \\
\text { cross sections }\end{array}$ & 15 & NaI Crystal & $\begin{array}{l}\text { Berkeley, } \\
\text { Bevatron }\end{array}$ & $\begin{array}{l}\text { P.A. Benioff, Phys, Rev. } \\
\text { 119, } 316(1960)\end{array}$ \\
\hline 173 & $p$ & $0.020-0.155$ & $\mathrm{Na}$ & $\sigma_{p, 1 p \operatorname{~In~}}$ & $\sim 30$ & $\begin{array}{l}\text { Radio-Chemical } \\
\text { Techniques }\end{array}$ & $\begin{array}{l}\text { Orsay, } \\
\text { Synchrocyclotron }\end{array}$ & $\begin{array}{l}\text { M. Gusakow et al. J. } \mathrm{J} \text {. } \\
\text { phys. radium } 2 \frac{22}{6} 636 \\
\text { (1961) }\end{array}$ \\
\hline 174 & $p$ & 0.096 & $\mathrm{Na}$ & $\sigma_{p, x p y}\left(E_{p}, 40^{\circ}\right)$ & 5 & $\begin{array}{l}\text { Range } \\
\text { Telescope }\end{array}$ & $\begin{array}{l}\text { Harvard, } \\
\text { Cyclotron }\end{array}$ & $\begin{array}{l}\text { K. Strauch and F. Titus, } \\
\text { Phys. Rev. 104, 191 (1956) }\end{array}$ \\
\hline 175 & $p$ & 0.185 & $\mathrm{Na}$ & $\begin{array}{l}\text { Nuclear level struc- } \\
\text { ture from proton } \\
\text { spectrum }\end{array}$ & - & Telescope & Upsala & $\begin{array}{l}\text { H. Tyrén and Th.A.J. Maris, } \\
\text { Nuclear Phys. 6, } 446 \text { (1958) }\end{array}$ \\
\hline
\end{tabular}




\begin{tabular}{|c|c|c|c|c|c|c|c|c|c|}
\hline No. & $\begin{array}{l}\text { Incident } \\
\text { Particle }\end{array}$ & $\begin{array}{l}\text { Incident } \\
\text { Particle } \\
\text { Energy (Gev) }\end{array}$ & Target & Measured Quantity & $\begin{array}{l}\text { Estimate of } \\
\text { Experimental } \\
\text { Error }(\%)\end{array}$ & Detector & $\begin{array}{l}\text { Installation and } \\
\text { Accelerator }\end{array}$ & References & Comments \\
\hline 176 & $\mathrm{p}$ & 5.7 & $\mathrm{Na}$ & $\begin{array}{l}{ }_{0} p, l p l n \text { and other } \\
\text { radio-chemical } \\
\text { cross sections }\end{array}$ & 15 & NaI Crystal & $\begin{array}{l}\text { Berkeley, } \\
\text { Bevatron }\end{array}$ & $\begin{array}{l}\text { P.A. Benioff, Phys. Rev. } \\
\text { I19, } 316 \text { (1960) }\end{array}$ & \\
\hline 177 & $\mathrm{p}$ & 0.096 & Mg & $\sigma_{p, x p y}\left(E_{p}, 40^{\circ}\right)$ & 5 & $\begin{array}{l}\text { Range } \\
\text { Telescope }\end{array}$ & $\begin{array}{l}\text { Harvard, } \\
\text { Cyclotron }\end{array}$ & $\begin{array}{l}\text { K. Strauch and F. Titus, } \\
\text { Phys. Rev. 104 191 (1956) }\end{array}$ & \\
\hline 178 & $\mathrm{p}$ & 0.185 & ME & $\begin{array}{l}\text { Nuclear level strue- } \\
\text { ture from proton } \\
\text { spectrum }\end{array}$ & - & Telescope & Upsala & $\begin{array}{l}\text { H. Tyrén and Th.A.J. Maris, } \\
\text { Nuclear Phys. 6, } 446 \text { (1958) }\end{array}$ & \\
\hline 179 & $\mathrm{p}$ & 0.061 & Al & $\begin{array}{l}\sigma_{\mathrm{pI}} \\
\sigma_{\mathrm{pX}}\end{array}$ & 6 & $\begin{array}{l}\text { Plastic } \\
\text { Scintillators }\end{array}$ & Minnesota & $\begin{array}{l}\text { v. Meyer, R.M. Eisberg, } \\
\text { and R.F. Carlson, Phys. } \\
\text { Rev. 117, } 1334 \text { (1960) }\end{array}$ & $\therefore$ \\
\hline 180 & $\mathrm{p}$ & 0.077 & Al & $\sigma_{\mathrm{px}}$ & $\sim 3$ & $\begin{array}{l}\text { Range } \\
\text { Telescope }\end{array}$ & $\begin{array}{l}\text { Harvard, } \\
\text { Cyclotron } \\
\end{array}$ & $\begin{array}{l}\text { R. Goloskie and K. } \\
\text { Strauch, Nuclear Phys. } \\
\text { 29, } 474 \text { (1962) }\end{array}$ & $\begin{array}{l}\text { Good geometry } \\
\therefore \quad \therefore .\end{array}$ \\
\hline 181 & $\mathrm{p}$ & $c .082$ & $A i$ & $\begin{array}{l}\text { Tritiun production } \\
\text { cross section }\end{array}$ & $\sim 10$ & $\begin{array}{l}\text { Radio-Chemical } \\
\text { Methods }\end{array}$ & $\begin{array}{c}\text { Orsay, } \\
\text { Synchrocyclotron }\end{array}$ & $\begin{array}{l}\text { C. Brun, M. Lefort, and } \\
\text { X. Tarrago, J. phys. } \\
\text { radium 23, } 167 \text { (1962) }\end{array}$ & \\
\hline 182 & $\mathrm{p} \cdot$ & $c .095$ & $A \equiv$ & $\sigma_{\mathrm{pX}}$ & $\sim 3$ & $\begin{array}{l}\text { Range } \\
\text { Telescope }\end{array}$ & $\begin{array}{l}\text { Harvard, } \\
\text { Cyclotron }\end{array}$ & $\begin{array}{l}\text { R. Goloskie and K. } \\
\text { Strauch, Nuclear Phys. } \\
\text { 29. } 474 \text { (1962) }\end{array}$ & Good geometry \\
\hline 183 & $p$ & 0.096 & Al & $\sigma_{p, x p y}\left(E_{p}, 40^{\circ}\right)$ & 5 & $\begin{array}{l}\text { Range } \\
\text { Telescope }\end{array}$ & $\begin{array}{l}\text { Harvard, } \\
\text { Cyclotron }\end{array}$ & $\begin{array}{l}\text { x. Strauch and F. Titus, } \\
\text { ?hys. Rev. 104, } 191 \text { (1956) }\end{array}$ & \\
\hline 184 & $\mathrm{p}$ & 0.113 & AI & $\sigma_{p \mathrm{x}}$ & $\sim 3$ & $\begin{array}{l}\text { Range } \\
\text { Telescope }\end{array}$ & $\begin{array}{l}\text { Harvard, } \\
\text { Cyclotron }\end{array}$ & $\begin{array}{l}\text { R. Goloskie and K. } \\
\text { Strauch, Nuclear Phys. } \\
\text { 29, } 474 \text { (1962) }\end{array}$ & Good geometry \\
\hline
\end{tabular}




\begin{tabular}{|c|c|c|c|c|c|c|c|c|c|}
\hline No. & $\begin{array}{l}\text { Incident } \\
\text { Particle }\end{array}$ & $\begin{array}{l}\text { Incident } \\
\text { Particle } \\
\text { Bnergy (Gev) }\end{array}$ & Target & Measured Quantity & $\begin{array}{l}\text { Estimate of } \\
\text { Experimental } \\
\text { Error }(\phi)\end{array}$ & Detector & $\begin{array}{l}\text { Installation and } \\
\text { Accelerator }\end{array}$ & References & Comments \\
\hline 185 & $\mathrm{p}$ & 0.133 & Al & ${ }^{\sigma}{ }^{\sigma}$ & $\sim 3$ & $\begin{array}{l}\text { Range } \\
\text { Telescope }\end{array}$ & $\begin{array}{l}\text { Harvard, } \\
\text { Cyclotron }\end{array}$ & $\begin{array}{l}\text { R. Goloskie and K. } \\
\text { Strauch, Nuclear Phys. } \\
\text { 29, } 474 \text { (1962) }\end{array}$ & Good geometry \\
\hline 186 & $\mathrm{p}$ & 0.142 & Al & $\stackrel{\sigma}{\mathrm{pT}}_{\dot{\sigma}_{\mathrm{p}, \mathrm{p}}}$ & $2-10$ & $\begin{array}{l}\text { Counter } \\
\text { Telescope }\end{array}$ & $\begin{array}{c}\text { Harwell, } \\
\text { Synchrocyclotron }\end{array}$ & $\begin{array}{l}\text { A.E. Taylor and E. Wood, } \\
\text { Nuclear Phys. 25, } 642 \\
\text { (1961) }\end{array}$ & Good geometry \\
\hline 187 & $\mathrm{p}$ & 0.143 & Al & $\begin{array}{l}\text { Neutron spectrum at } \\
\text { zero degrees }\end{array}$ & $3-10$ & $\begin{array}{l}\text { Neutron Time- } \\
\text { of-Fligit } \\
\text { Spectrometer }\end{array}$ & $\begin{array}{c}\text { Harwell; } \\
\text { Synchrocyclotron }\end{array}$ & $\begin{array}{l}\text { P.H. Bowen et al., Nuclear } \\
\text { Phys. 30, } 475 \frac{1962)}{(196)}\end{array}$ & \\
\hline 188 & $\mathrm{p}$ & 0.150 & Al & $\begin{array}{l}\text { Tritium production } \\
\text { cross section }\end{array}$ & $\sim 10$ & $\begin{array}{l}\text { Radio-Chemical } \\
\text { Methods }\end{array}$ & $\begin{array}{l}\text { Orsay, } \\
\text { Symchrocyclotron }\end{array}$ & $\begin{array}{l}\text { C. Brun, M. Lefort, and } \\
\text { X. Tarrago, J. phys. } \\
\text { radium 23, } 167 \text { (1962) }\end{array}$ & \\
\hline 189 & $\mathrm{p}$ & 0.150 & Al & $\begin{array}{l}\sigma_{p, 3 p l n} \\
\sigma_{p, 3 p 3 n}\end{array}$ & 5 & - & $\begin{array}{l}\text { Institute for } \\
\text { Nuclear Problems }\end{array}$ & $\begin{array}{l}\text { Yu.D. Prokoshkin and } \\
\text { A.A. Tiapkin, Soviet Phys.- } \\
\text { JETP } 5,148(1957)\end{array}$ & \\
\hline 190 & $\mathrm{p}$ & 0.150 & Al & $\begin{array}{l}\text { Energy distribution } \\
\text { of neutrons emitted } \\
\text { in backward direction }\end{array}$ & $\sim 15$ & $\begin{array}{l}\text { Recoil } \\
\text { Protons } \\
\text { in } \\
\text { Emulsions }\end{array}$ & $\begin{array}{l}\text { Harwell, } \\
\text { Symchrocyclotron }\end{array}$ & $\begin{array}{l}\text { D. Skyrme, Nuclear Phys. } \\
\text { 25, } 177 \text { (1962) }\end{array}$ & \\
\hline 191 & $\mathrm{p}$ & 0.155 & $\mathrm{Al}^{27}$ & $\sigma_{p, 3 p 3 n}$ & 3 & $\begin{array}{l}\text { Radio-Chemical } \\
\text { Techniques }\end{array}$ & $\begin{array}{l}\text { Orsay, } \\
\text { Synchrocyclotron. }\end{array}$ & $\begin{array}{l}\text { M. Nguyen-Long-Den, } \\
\text { Compt. rend. } 253 \\
2919 \text { (1961) }\end{array}$ & . \\
\hline 192 & p. & 0.180 & $\mathrm{AI}$ & $\sigma_{\mathrm{pX}}$ & 3 & $\begin{array}{l}\text { Range } \\
\text { Separator }\end{array}$ & $\begin{array}{l}\text { Upsala, } \\
\text { Cyclotron }\end{array}$ & $\begin{array}{l}\text { A. Johansson, U. Svanberg, } \\
\text { and 0. Sundbert, Arkiv } \\
\text { Fysik 19, } 527 \text { (1961) }\end{array}$ & Poor geometry \\
\hline
\end{tabular}




\begin{tabular}{|c|c|c|c|c|c|c|c|c|c|}
\hline No. & $\begin{array}{l}\text { Incident } \\
\text { Particle }\end{array}$ & $\begin{array}{l}\text { Incident } \\
\text { Particle } \\
\text { Energy (Gev) }\end{array}$ & Targəড & Measured quantity & $\begin{array}{l}\text { Estimate of } \\
\text { Experimental } \\
\text { Error }(\%)\end{array}$ & Detectcr & $\begin{array}{l}\text { Installation and } \\
\text { Accelerator }\end{array}$ & References & Comments \\
\hline 193 & $\mathrm{p}$ & 0.185 & Al & $\begin{array}{l}\text { Nuclear level struc- } \\
\text { ture from proton } \\
\text { spectrum }\end{array}$ & - & Telescore & Upsala & $\begin{array}{l}\text { H. Tyrén and Th.A.J. Maris } \\
\text { Nuclear Phys. } 6,446(1958)\end{array}$ & \\
\hline 194 & $p$ & $0.082-0.426$ & $\mathrm{~A}^{27}$ & $\sigma_{p, 3 p l n}$ & $2-10$ & $\begin{array}{l}\text { Radio-Chemical } \\
\text { Techniques }\end{array}$ & $\begin{array}{l}\text { Chicago, } \\
\text { Eynchrocyclotron }\end{array}$ & $\begin{array}{l}\text { H.P. Yule and A. Turkevich, } \\
\text { Phys. Rev. 118, } 1591(1960)\end{array}$ & \\
\hline 195 & $\mathrm{p}$ & 0.260 & AI & $\begin{array}{l}\sigma_{p}, 3 p 1 n \\
\sigma_{p}, 3 p 3 n\end{array}$ & 5 & - & $\begin{array}{l}\text { Institute for } \\
\text { Nuclear Problems }\end{array}$ & $\begin{array}{l}\text { Yu.D. Prokoshkin and A.A. } \\
\text { Tiapkin, Soviet Phys..- } \\
\text { JETP } 5 \text {, } 148 \text { (1957) }\end{array}$ & \\
\hline 196 & $\mathrm{P}$ & 0.202 to 0.342 & Al & $\begin{array}{l}\sigma_{p}, 5 p 5 n, \sigma_{p}, 3 p 1 n \\
\text { reiative to } \sigma^{\circ} \text { of } \\
c^{12}(p, p n) c^{11}\end{array}$ & $2-10$ & Counters & $\begin{array}{l}\text { Iiverpool, } \\
\text { Synchrocyclotron }\end{array}$ & $\begin{array}{l}\text { v. Parikh, Ijuclear Phys. } \\
\text { 12. } 638(1960)\end{array}$ & \\
\hline 197 & $\mathrm{p}$ & 0.340 & AI & Neutron yiela & 10 & $\begin{array}{l}\mathrm{MnSO}_{4} \\
\text { Solutior: }\end{array}$ & $\begin{array}{l}\text { Perkeley and } \\
\text { Chicago } \\
\text { Cyclotrons }\end{array}$ & $\begin{array}{l}\text { W.E. Cranda:1 and G.P. } \\
\text { Millburn, J. Appl. Phys. } \\
\text { 2C. } 698(1958) \\
\end{array}$ & \\
\hline 198 & $\mathrm{p}$ & 0.350 & Al & $\begin{array}{l}\sigma_{\mathrm{p}}, 3 \mathrm{pln} \\
\sigma_{\mathrm{p}, 3 \mathrm{p} 3 \mathrm{n}}\end{array}$ & 5 & - & $\begin{array}{l}\text { Institute for } \\
\text { Nuclear Problems }\end{array}$ & $\begin{array}{l}\text { YL.D. Prokoshkin and A.A. } \\
\text { Tiapkin, Soviet Phys。 - } \\
\text { JETP 5, } 148 \text { (1957) }\end{array}$ & \\
\hline 199 & $\mathrm{p}$ & $0.130-0.660$ & $\mathrm{~A}_{1}^{27}$ & $\sigma_{p, 1 p 1 n^{+}}$ & $3-10$ & $\begin{array}{l}\text { Radio-Chemical } \\
\text { Techniques }\end{array}$ & $\begin{array}{l}\text { Joint Institute } \\
\text { for Nuclear } \\
\text { Fesearch, } \\
\text { Synchrocyclotron }\end{array}$ & $\begin{array}{l}\text { M.Ya. Kuzne-sova, V.N. } \\
\text { Pckrovski, and V.N. } \\
\text { Rybakov, Sorriet Phys.- } \\
\text { JETP 42, 1451 (1962) }\end{array}$ & \\
\hline & $\mathrm{p}$ & 0.450 & $\mathrm{Al}$ & $\begin{array}{c}\dot{\sigma}_{p}, x_{\pi^{+}}+\left(E_{\pi^{+}}, \psi_{\pi^{+}}\right) \\
\sigma_{p}, x_{\pi^{-}-y}\left(E_{\pi^{-}}, \psi_{\pi^{-}}\right) \\
\text {with } \psi_{\pi^{ \pm}}=21.5^{\circ}, 60^{\circ}\end{array}$ & $2-40$ & $\begin{array}{l}\text { Counter } \\
\text { Telescope }\end{array}$ & $\begin{array}{l}\text { Chicago, } \\
\text { Synchrocyclotron }\end{array}$ & $\begin{array}{l}\text { E. Lillethur, Phys. Rev. } \\
125,665 \text { (1962) }\end{array}$ & Good geometry \\
\hline
\end{tabular}




\begin{tabular}{|c|c|c|c|c|c|c|c|c|c|}
\hline No. & $\begin{array}{l}\text { Incident } \\
\text { Particle }\end{array}$ & $\begin{array}{l}\text { Incident } \\
\text { Particle } \\
\text { Bnergy (Gev) }\end{array}$ & Target & Measured Quantity & $\begin{array}{l}\text { Estimate of } \\
\text { Experimental } \\
\text { Error }(\%)\end{array}$ & Detector & $\begin{array}{l}\text { Installation and } \\
\text { Accelerator }\end{array}$ & References & Comments \\
\hline 201 & $p$ & 0.450 & $A I$ & $\begin{array}{l}\sigma_{p}, 3 p l n \\
\sigma_{p, 3 p 3 n}\end{array}$ & 5 & - & $\begin{array}{l}\text { Institute for } \\
\text { Nuclear Problems }\end{array}$ & $\begin{array}{l}\text { Yu.D. Prokoshkin and A.A. } \\
\text { Tiapkin, Soviet Phys.- } \\
\text { JETP } 5,148 \text { (1957) }\end{array}$ & \\
\hline 202 & $\mathrm{p}$ & $0.185-0.870$ & AI & ${ }^{\sigma} \mathrm{pX}$ & 10 & - & - & $\begin{array}{l}\text { G.P. Millburn et al., } \\
\text { Phys. Rev. 95, } 1268 \text {. } \\
(1954)\end{array}$ & A compilation \\
\hline 203 & $\mathrm{p}$ & 0.560 & AI & $\begin{array}{l}\sigma_{p}, 3 p l n \\
\sigma_{p}, 3 p 3 n\end{array}$ & 5 & - & $\begin{array}{l}\text { Institute for } \\
\text { Nuclear Problems }\end{array}$ & $\begin{array}{l}\text { Yu.D. Prokoshkin and } \\
\text { A.A. Tiapkin, Soviet } \\
\text { Phys. JETP 5, 148 } \\
\text { (1957) }\end{array}$ & . \\
\hline 204 & $\mathrm{p}$ & 0.591 & AI & $\sigma_{p, 3 p l n}$ & 5 & $\begin{array}{l}\text { Radio-Chemical } \\
\text { Methods }\end{array}$ & $\begin{array}{c}\text { CERN, } \\
\text { Synchrocyclotron }\end{array}$ & $\begin{array}{l}\text { K. Goebel et al., Nuclear } \\
\text { Phys. } 24, \frac{1}{28}(1961)\end{array}$ & \\
\hline 205 & $\mathrm{p}$ & 0.653 & Al & $\begin{array}{l}\sigma_{\mathrm{pT}} \\
\sigma_{\mathrm{pX}}\end{array}$ & 5 & Telescope & $\begin{array}{l}\text { Institute for } \\
\text { Nuclear Problems, } \\
\text { Synchrocyclotron }\end{array}$ & $\begin{array}{l}\text { V.I. Moskalev and B.V. } \\
\text { Gavrilovskii, Soviet } \\
\text { Phys. Doklady } 1,607 \text { (1956) }\end{array}$ & $\begin{array}{l}\text { Good and poor } \\
\text { geometry }\end{array}$ \\
\hline 206 & $\mathrm{p}$ & 0.660 & AI & $\sigma_{p, x \pi^{+} y}\left(E_{\pi^{+}}, 45^{\circ}\right)$ & 10 & $\begin{array}{l}\text { Magneti: } \\
\text { Method } \\
\text { Telescope }\end{array}$ & $\begin{array}{l}\text { Institute for } \\
\text { Nuclear Problems, } \\
\text { Synchrocyclotron }\end{array}$ & $\begin{array}{l}\text { A.G. Meshkovskii et al., } \\
\text { Soviet Phys. JETP } \frac{4}{4} \text {, } \\
842 \text { (1957) }\end{array}$ & \\
\hline 207 & $\mathrm{p}$ & 0.6610 & AI & $\sigma_{p, x \pi^{-} y}\left(E_{\pi^{-}}, 45^{\circ}\right)$ & 20 & - & $\begin{array}{l}\text { Joint Institute } \\
\text { for Nuclear } \\
\text { Research, } \\
\text { Synchrocyclotron }\end{array}$ & $\begin{array}{l}\text { A.G. Meshkovskii, Ia.Ia. } \\
\text { Shalamov, and V.A. } \\
\text { Shebanov, Soviet Phys.- } \\
\text { JETP } 6,463 \text { (1958) }\end{array}$ & . \\
\hline 208 & $\mathrm{p}$ & 0.660 & Al & $\begin{array}{l}\sigma_{p, 3 p l n} \\
\sigma_{p, 3 p 3 n}\end{array}$ & 5 & - & $\begin{array}{l}\text { Institute for } \\
\text { Nuclear Problems }\end{array}$ & $\begin{array}{l}\text { Yu.D.Prokoshkin and A.A. } \\
\text { Tiapkin, Soviet Phys. - } \\
\text { JETP 5, } 148 \text { (1957) }\end{array}$ & \\
\hline
\end{tabular}




\begin{tabular}{|c|c|c|c|c|c|c|c|c|c|}
\hline No. & $\begin{array}{l}\text { Incident } \\
\text { Particle }\end{array}$ & $\begin{array}{c}\text { In:ident } \\
\text { Particle } \\
\text { Energy (Gev) }\end{array}$ & Target & Measured quantity & $\begin{array}{l}\text { Estimate of } \\
\text { Experimental } \\
\text { Error }(\phi)\end{array}$ & Detector & $\begin{array}{l}\text { Installation and } \\
\text { Accelerator }\end{array}$ & References & Comments \\
\hline 209 & $p$ & 0.900 & Al & $\begin{array}{l}\sigma_{\mathrm{pT}} \\
\sigma_{\mathrm{pX}}\end{array}$ & $5-10$ & Telescope & $\begin{array}{l}\text { Birmingham, } \\
\text { Synchrotron }\end{array}$ & $\begin{array}{l}\text { N.E. Booth et al. } \\
\text { Proc. Phys. Soc. A(London) } \\
\text { I0, } 209 \text { (1957) }\end{array}$ & $\begin{array}{l}\text { Good and poor } \\
\text { geometry }\end{array}$ \\
\hline 210 & $\mathrm{p}$ & 0.930 & Al & 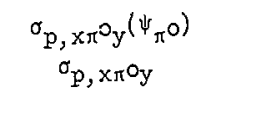 & $20-40$ & $\begin{array}{l}\text { Telescope and } \\
\text { Total Absorp- } \\
\text { tion Cerenkov } \\
\text { Counter }\end{array}$ & Birmingham & $\begin{array}{l}\text { h.M. Segar and R. } \\
\text { Rubinstein, Nuclear } \\
\text { Phys. 14, 222 (1959) }\end{array}$ & \\
\hline 211 & $\mathrm{p}$ & 1.0 & A.I & Production of $\mathrm{Be}^{7}$ & $\sim 25$ & $\begin{array}{l}\text { Radio-Chemical } \\
\text { Techniques }\end{array}$ & $\begin{array}{l}\text { Brookhaven, } \\
\text { Cosmotron }\end{array}$ & $\begin{array}{l}\text { E. Baker, G. Friedlander, } \\
\text { and J. Hudis, Phys. Rev. } \\
\underline{112} 1319 \text { (1958) }\end{array}$ & \\
\hline 212 & $\underline{p}$ & 1.4 & A. & Producition of $\mathrm{Be}^{7}$ & $\sim 25$ & $\begin{array}{l}\text { Radio-Chemical } \\
\text { Techniques }\end{array}$ & $\begin{array}{l}\text { Brookhaven, } \\
\text { Cosmotron }\end{array}$ & $\begin{array}{l}\text { E. Baker, G. Friedlander, } \\
\text { and J. Hudis, Phys. Rev. } \\
\text { 112, } 1319 \text { (1958) }\end{array}$ & \\
\hline 213 & $\mathrm{p}$ & 2 & $\begin{array}{c}\text { Al } \\
\text { (thick) }\end{array}$ & $\sigma_{p,-p \ln }$ & 4 & $\begin{array}{l}\text { Telescope for } \\
\text { p Flux. Scin- } \\
\text { tillators for } \\
\mathrm{C}^{11}\end{array}$ & $\begin{array}{l}\text { Brookhaven, } \\
\text { Cosmotron }\end{array}$ & $\begin{array}{l}\text { J.B. Curming, G. Friedlander, } \\
\text { and C.E. Swartz, Phys. Rev. } \\
\text { 111, } 1386(1958)\end{array}$ & \\
\hline 214 & $\mathrm{p}$ & 2.2 & Al & Production of $\mathrm{Be}^{7}$ & $\sim 25$ & $\begin{array}{l}\text { Radio-Chemical } \\
\text { Techniques }\end{array}$ & $\begin{array}{l}\text { Brookhaven, } \\
\text { Cosmotron }\end{array}$ & $\begin{array}{l}\text { E. Baker; G. Friedlander, } \\
\text { and J. Hudis, Phys. Rev. } \\
\text { I12 } 1319 \text { (1958) }\end{array}$ & \\
\hline 215 & $\mathrm{p}$ & 3.0 & $\mathrm{Al}$ & Producition of $\mathrm{Be}^{7}$ & $\sim 25$ & $\begin{array}{l}\text { Radio-Chemical } \\
\text { T'echniques }\end{array}$ & $\begin{array}{l}\text { Brookhaven, } \\
\text { Cosmotron }\end{array}$ & $\begin{array}{l}\text { E. Baker, G. Friedlander, } \\
\text { and J. Hudis, Phys. Rev. } \\
112,1319 \text { (1958) }\end{array}$ & \\
\hline 216 & $\mathrm{p}$ & 3 & $\begin{array}{c}\mathrm{Al} \\
\text { (thick) }\end{array}$ & $\sigma_{p, 1 p l n}$ & 4 & $\begin{array}{l}\text { Telescope for } \\
\text { p Flux. Scin- } \\
\text { tillators for } \\
\text { C }^{11}\end{array}$ & $\begin{array}{l}\text { Brookhaven, } \\
\text { Cosmotron }\end{array}$ & $\begin{array}{l}\text { ․B. Cumning, G. Friedlander, } \\
\text { and C.E. Swartz, Phys. Rev. } \\
\text { I111, } 1386 \text { (1958) }\end{array}$ & \\
\hline
\end{tabular}




\begin{tabular}{|c|c|c|c|c|c|c|c|c|c|}
\hline No. & $\begin{array}{l}\text { Incident } \\
\text { Particle }\end{array}$ & $\begin{array}{l}\text { Incident } \\
\text { Particle } \\
\text { Energy (Gev) }\end{array}$ & Target & Measured Quantity & $\begin{array}{l}\text { Estimate of } \\
\text { Experimental } \\
\text { Error }(\phi)\end{array}$ & Detector & $\begin{array}{l}\text { Installation and } \\
\text { Accelerator }\end{array}$ & References & Comments \\
\hline 217 & $\mathrm{p}$ & $3 \mathrm{Gev} / \mathrm{c}$ & Al & $\begin{array}{l}\sigma_{p, p} \\
\sigma_{p X}\end{array}$ & $5-10$ & $\begin{array}{l}\text { Scintillation } \\
\text { Counters }\end{array}$ & $\begin{array}{l}\text { Berkeley, } \\
\text { Bevatron }\end{array}$ & $\begin{array}{l}\text { M.J. Longo and B.J. Moyer, } \\
\text { Phys. Rev. 125, } 701 \\
\text { (1962) }\end{array}$ & $\begin{array}{l}\text { Good and poor } \\
\text { geometry }\end{array}$ \\
\hline 218 & $\mathrm{p}$ & 5.7 & AI & $\begin{array}{l}\sigma_{\mathrm{p}, 1 p l n} \text { andi other } \\
\text { radio-chemical } \\
\text { cross sections }\end{array}$ & 15 & NaI Crystal & $\begin{array}{l}\text { Berkeley, } \\
\text { Bevatron }\end{array}$ & $\begin{array}{l}\text { P.A. Benioff, Phys. Rev. } \\
\text { 119. } 316(1960)\end{array}$ & \\
\hline 219 & $\mathrm{p}$ & $0.4-17$ & $\mathrm{Al}^{2 \mathrm{~T}}$ & $\begin{array}{l}\text { Ratio of } \mathrm{Al}^{27}(\mathrm{p}, 3 \mathrm{pn}) \\
\mathrm{Na}^{24} / \mathrm{C}^{12}(\mathrm{p}, \mathrm{pn}) \mathrm{C}^{11} \\
\text { cross sections }\end{array}$ & 5 & $\begin{array}{l}\text { Scintillation } \\
\text { and. Propor- } \\
\text { tional Counters }\end{array}$ & $\begin{array}{l}\text { Brookhaven, } \\
\text { Symchrotron }\end{array}$ & $\begin{array}{l}\text { J.B. Cunming et al.; } \\
\text { Phys. Rev. 128, } \frac{13}{2392} \\
\text { (1962) }\end{array}$ & \\
\hline 220 & $\mathrm{p}$ & 10 & $\mathrm{Al}$ & $\begin{array}{l}\text { Momentum spectra of } \\
\text { protons, } \pi^{+}, \pi^{-} \text {, at } \\
\text { various angles from } \\
4.75^{\circ} \text { to } 20^{\circ} \text {. Produc- } \\
\text { tion ratios of } \mathrm{K}^{+} / \pi^{+} \text {, } \\
\mathrm{K}^{-} / \pi^{-}, \mathrm{F} / \pi^{\circ} \text { at } \\
4.75^{\circ} \text { and } 9^{\circ} \text {. }\end{array}$ & - & $\begin{array}{l}\text { Magnetic Deflec- } \\
\text { tion and Focus- } \\
\text { ing Gas Cerenkov } \\
\text { Counters }\end{array}$ & $\begin{array}{l}\text { Brookhaven, } \\
\text { Synchrotron }\end{array}$ & $\begin{array}{l}\text { W.F. Baker et al., } \\
\text { Phys. Rev. Letters } 7 \\
\text { 101 (1961) }\end{array}$ & \\
\hline 221 & $p$ & 20 & Al & $\begin{array}{l}\text { Momentum spectra of } \\
\text { protons, } \pi^{+}, \pi^{-} \text {, at } \\
\text { various angles from } \\
4.75^{\circ} \text { to } 20^{\circ} \text {. Produc- } \\
\text { tion ratios of } \mathrm{K}^{+} / \pi^{+} \text {, } \\
\mathrm{K}^{-} / \pi^{-}, \mathrm{p} / \pi^{-} \text {at } \\
4.75^{\circ} \text { and } 9^{\circ} \text {. }\end{array}$ & - & $\begin{array}{l}\text { Magnetic Deflec- } \\
\text { tion and Focus- } \\
\text { ing Gas } \\
\text { Cerenkov } \\
\text { Counters }\end{array}$ & $\begin{array}{l}\text { Brookhaven, } \\
\text { Synchrotron }\end{array}$ & $\begin{array}{l}\text { W.F. Baker et al.; } \\
\text { Phys. Rev. Letters I, } \\
101 \text { (1961) }\end{array}$ & \\
\hline 222 & $\mathrm{p}$ & 25 & Al & $\begin{array}{l}\text { Momentum spectra of } \\
\text { protons, } \pi^{+}, \pi^{-} \text {, at } \\
\text { various angles from } \\
4.75^{\circ} \text { to } 20^{\circ} \text {. Produc- }- \\
\text { tion ratios of } \mathrm{K}^{+} / \pi^{+} \text {, } \\
\mathrm{K}^{-} / \pi^{-} \text {p } / \pi^{-} \text {at } \\
4.75^{\circ} \text { and } 9^{\circ} \text {. }\end{array}$ & - & $\begin{array}{l}\text { Magnetic Deflec- } \\
\text { tion and Focus- } \\
\text { ing Gas } \\
\text { Cerenkov } \\
\text { Counters }\end{array}$ & $\begin{array}{l}\text { Brookhaven, } \\
\text { Synchrotron }\end{array}$ & $\begin{array}{l}\text { W.F. Baker et al., } \\
\text { Phys. Rev. Letters I } \\
101 \text { (1961) }\end{array}$ & . \\
\hline
\end{tabular}




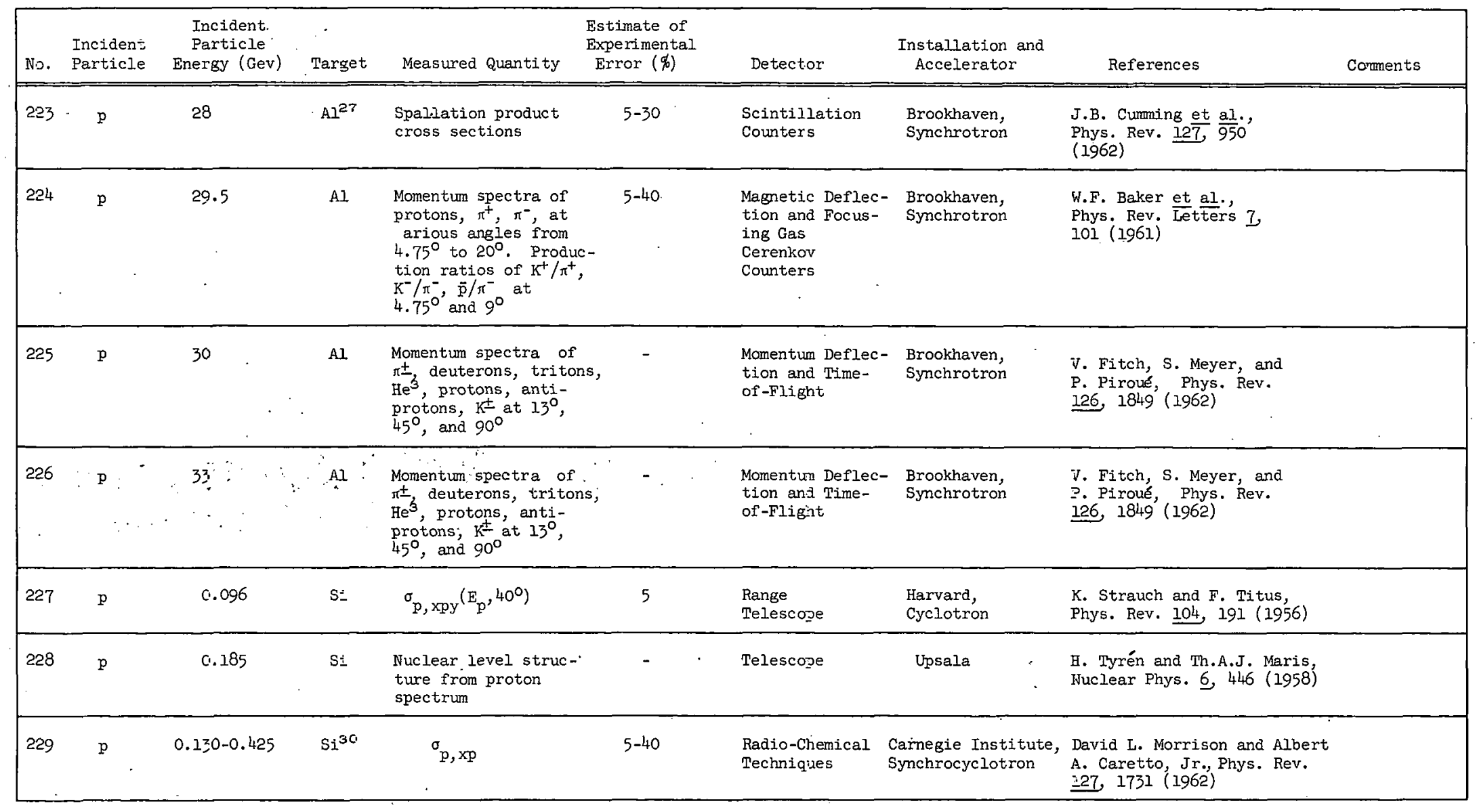




\begin{tabular}{|c|c|c|c|c|c|c|c|c|}
\hline No. & $\begin{array}{l}\text { Incident } \\
\text { Particle }\end{array}$ & $\begin{array}{l}\text { Incident } \\
\text { Particle } \\
\text { Energy (Gev) }\end{array}$ & Target & Measured Quantity & $\begin{array}{l}\text { Estimate of } \\
\text { Experimental } \\
\text { Error }(\phi)\end{array}$ & Detector & $\begin{array}{l}\text { Installation and } \\
\text { Accelerator }\end{array}$ & References \\
\hline 230 & $\mathrm{p}$ & 0.660 & $\mathrm{Si}$ & $\sigma_{p, 1 \pi^{+}}$ & - & $\begin{array}{l}\text { Radio-Chemical } \\
\text { Methods }\end{array}$ & $\begin{array}{l}\text { Institute of Geo- } \\
\text { chemistry and } \\
\text { Analytical Chemistry, } \\
\text { USSR }\end{array}$ & $\begin{array}{l}\text { A.K. Lavrukhina, Soviet } \\
\text { Phys. Doklady } 4,1317 \\
\text { y, } \\
\text { (1959) }\end{array}$ \\
\hline 232 & $\mathrm{p}$ & 0.096 & $P$ & $\sigma_{p, x p y}\left(E_{p}, 40^{\circ}\right)$ & 5 & $\begin{array}{l}\text { Range } \\
\text { Telescope }\end{array}$ & $\begin{array}{l}\text { Harvard, } \\
\text { Cyclotron }\end{array}$ & $\begin{array}{l}\text { K. Strauch and F. Tistus, } \\
\text { Phys. Rev. 104, 19? } \\
\text { (1956) }\end{array}$ \\
\hline 232 & $\dot{p}$ & 0.125 & $\mathrm{P}$ & $\begin{array}{l}\text { Nuclear level struc- } \\
\text { ture from proton } \\
\text { spectrum }\end{array}$ & - & Telescope & Upsala & $\begin{array}{l}\text { H. Tyrén and Th.A.J. Maris, } \\
\text { Nuclear Phys. } 6 \text {, } 446 \\
\text { (1958). }\end{array}$ \\
\hline 233 & $\mathrm{p}$ & $0.230-c .425$ & $P^{32}$ & $\sigma_{p, x p}$ & $5-40$ & $\begin{array}{l}\text { Radio-Chemical } \\
\text { Techniques }\end{array}$ & $\begin{array}{l}\text { Carnegie In- } \\
\text { stitute, } \\
\text { Synchrocyclotron }\end{array}$ & $\begin{array}{l}\text { D. L. Morrison and A.A. Caretto, } \\
\text { Jr., Phys. Rev. 127, } 1731 \\
\text { (1962) }\end{array}$ \\
\hline 234 & $\mathrm{p}$ & 0.096 & s & $\sigma_{p, x p y}\left(E_{p}, 40^{\circ}\right)$ & 5 & $\begin{array}{l}\text { Range } \\
\text { Telesccpe }\end{array}$ & $\begin{array}{l}\text { Harvard, } \\
\text { Cyclotron }\end{array}$ & $\begin{array}{l}\text { K. Strauch and F. Titus, } \\
\text { Phys. Rev. 104, } 191 \\
\text { (1956) }\end{array}$ \\
\hline 235 & $\mathrm{p}$ & 0.185 & $\mathrm{~s}$ & $\begin{array}{l}\text { Nuclear level struc- } \\
\text { ture from proton } \\
\text { spectrum }\end{array}$ & - & Telesccpe & Upsala & $\begin{array}{l}\text { H. Tyrén and Th.A.J. Maris, } \\
\text { Nuclear Phys. } 6,446 \text { (1958) }\end{array}$ \\
\hline 236 & $\mathrm{p}$ & $0.130-0.425$ & $s^{32}$ & $\sigma_{p, x p}$ & $5-40$ & $\begin{array}{l}\text { Radio-Chemical } \\
\text { Techniçues }\end{array}$ & $\begin{array}{l}\text { Carnegie } \\
\text { Institute, } \\
\text { Synchrocyclotron }\end{array}$ & $\begin{array}{l}\text { D. L. Morrison and A.A. } \\
\text { Caretto, Jr., Phys. Rev. } \\
\text { 127, } 1731 \text { (1962) }\end{array}$ \\
\hline 237 & $\mathrm{p}$ & $0.130-0.425$ & $\mathrm{~s}$ & $\sigma_{p, x p}$ & $5-40$ & $\begin{array}{l}\text { Radio-Chemical } \\
\text { Techniques }\end{array}$ & $\begin{array}{l}\text { Carnegie } \\
\text { Institute, } \\
\text { Symchrocyclotron }\end{array}$ & $\begin{array}{l}\text { D.L. Morrison and A.A. } \\
\text { Caretto, Jr., Phys. Rev. } \\
127,1731 \text { (1962) }\end{array}$ \\
\hline
\end{tabular}




\begin{tabular}{|c|c|c|c|c|c|c|c|c|c|}
\hline No. & $\begin{array}{l}\text { Incident } \\
\text { Particle }\end{array}$ & $\begin{array}{l}\text { Incident } \\
\text { Particle } \\
\text { Energy (Gev) }\end{array}$ & Target & Measured Quantity & $\begin{array}{l}\text { Estimate of } \\
\text { Experimental } \\
\text { Error }(\%)\end{array}$ & Detector & $\begin{array}{l}\text { Installation and } \\
\text { Accelerator }\end{array}$ & References & Comments \\
\hline 238 & $p$ & 0.185 & $\mathrm{C} \ell$ & $\begin{array}{l}\text { Nuclear level struc- } \\
\text { ture from proton } \\
\text { spectrum }\end{array}$ & - & Telescope & Upsala & $\begin{array}{l}\text { H. Tyrén and Th.A.J. Maris, } \\
\text { Nuclear Phys. } 6,446 \text { (1958) }\end{array}$ & \\
\hline 239 & $p$ & 0.185 & K & $\begin{array}{l}\text { Nuclear level struc- } \\
\text { ture from proton } \\
\text { spectrum }\end{array}$ & - & Telescope & Upsala & $\begin{array}{l}\text { H. Tyrén and Th.A.J. Maris, } \\
\text { Nuclear Phys. 6, } 446 \text { (1958) }\end{array}$ & \\
\hline 240 & $\mathrm{p}$ & 0.185 & A & $\begin{array}{l}\text { Nuclear level struc- } \\
\text { ture from proton } \\
\text { spectrum }\end{array}$ & - & Telescope & Upsala & $\begin{array}{l}\text { H. Tyrén and Th.A.J. Maris, } \\
\text { Nuclear Phys. } 6,446 \text { (1958) }\end{array}$ & \\
\hline 241 & $\mathrm{p}$ & 0.180 & $\mathrm{Ca}$ & $\sigma_{p X}$ & 3 & $\begin{array}{l}\text { Range } \\
\text { Separator }\end{array}$ & $\begin{array}{l}\text { Upsala, } \\
\text { Cyclotron }\end{array}$ & $\begin{array}{l}\text { A. Johansson, U. Svanberg, } \\
\text { and O. Sundberg, Arkiv } \\
\text { Fysik 19, } 527 \text { (1961) }\end{array}$ & Poor geometry \\
\hline 242 & $\mathrm{p}$ & 0.185 & $\mathrm{Ca}$ & $\begin{array}{l}\text { Nuclear shell struc- } \\
\text { ture from } \\
\sigma_{p, Z p}\left(E_{p 1}, E_{p 2}, \psi_{p l}, \psi_{p 2}\right)\end{array}$ & ) & $\begin{array}{l}\text { Double Tele- } \\
\text { scope Renge } \\
\text { Method for } \\
\text { Energy }\end{array}$ & Upsala & $\begin{array}{l}\text { H. Tyrén, P. Hillman, and } \\
\text { Th.A.J. Maris, Nuclear } \\
\text { Fhys. I } 10 \text { (1958) }\end{array}$ & . \\
\hline 243 & $\mathrm{p}$ & $0.130-0.425$ & $v^{51}$ & ${ }^{\sigma} p, x p$ & $5-40$ & $\begin{array}{l}\text { Radio-Cr:emical } \\
\text { Techniques }\end{array}$ & $\begin{array}{l}\text { Carnegie } \\
\text { Institute, } \\
\text { Symchrocyclotron }\end{array}$ & $\begin{array}{l}\text { I. L. Morrison and A.A. } \\
\text { Caretto, Jr., Phys. Rev. } \\
127,1731 \text { (i962) }\end{array}$ & \\
\hline 244 & $\mathrm{p}$ & $0.020-0.150$ & $\mathrm{Mn}$ & $\sigma_{p, 1 p l n}$ & 30 & $\begin{array}{l}\text { Radio-Cremical } \\
\text { Techniques }\end{array}$ & $\begin{array}{c}\text { Orsay, } \\
\text { Synchrocyclotron }\end{array}$ & $\begin{array}{l}\text { N. Gusakow: Ann. phys. } \\
\text { (Paris) I. } 67 \text { (1962) }\end{array}$ & \\
\hline 245 & $\mathrm{p}$ & $0.020-0.155$ & $\mathrm{Mn}$ & $\cdot \sigma_{p, l p l n}$ & $\sim 30$ & $\begin{array}{l}\text { Radio-Chemical } \\
\text { Techniques }\end{array}$ & $\begin{array}{c}\text { Orsay, } \\
\text { Synchrocyclotron }\end{array}$ & $\begin{array}{l}\text { N. Gusakow et al., J. } \\
\text { chys. radium } 2 \frac{\mathrm{J}}{2}, 636 \\
\text { (1961) }\end{array}$ & \\
\hline
\end{tabular}




\begin{tabular}{|c|c|c|c|c|c|c|c|c|}
\hline No. & $\begin{array}{l}\text { Incident } \\
\text { Particle }\end{array}$ & $\begin{array}{l}\text { Incident } \\
\text { Particle } \\
\text { Inergy (Gev) }\end{array}$ & Target & Measured Quantity & $\begin{array}{l}\text { Estimate of } \\
\text { Experimental } \\
\text { Error }(\phi)\end{array}$ & Detector & $\begin{array}{l}\text { Installation and } \\
\text { Accelerator }\end{array}$ & References \\
\hline 246 & $p$ & 0.061 & $\begin{array}{c}\mathrm{Fe} \\
\text { (thin) }\end{array}$ & $\sigma_{\mathrm{pT}}, \quad \sigma_{\mathrm{pX}}$ & 6 & $\begin{array}{l}\text { Plastic } \\
\text { Scintillators }\end{array}$ & Minnesota & $\begin{array}{l}\text { v. Meyer, R.M. Eisberg, } \\
\text { and R.F. Carlson, Phys. } \\
\text { Rev. 117, } 1334 \text { (1960) }\end{array}$ \\
\hline 247 & $\mathrm{p}$ & 0.150 & $\mathrm{Fe}$ & $\begin{array}{l}\text { Tritium production } \\
\text { cross sections }\end{array}$ & $\sim 10$ & $\begin{array}{l}\text { Radio-Chemical } \\
\text { Methods }\end{array}$ & $\begin{array}{l}\text { Orsay, } \\
\text { Synchrocyclotron }\end{array}$ & $\begin{array}{l}\text { C. Brun, M. Lefort, and } \\
\text { X. Tarrago, J. phys. } \\
\text { radium 23, } 167 \text { (1962) }\end{array}$ \\
\hline 248 & $\mathrm{p}$ & 0.180 & $\mathrm{Fe}$ & $\sigma_{p X}$ & 3 & $\begin{array}{l}\text { Range } \\
\text { Separators }\end{array}$ & $\begin{array}{l}\text { Upsala, } \\
\text { Cyclotron }\end{array}$ & $\begin{array}{l}\text { A. Johansson, U. Svanberg, Poor geometry } \\
\text { and o. Sundberg, Arkiv } \\
\text { Fysik 19, } 527 \text { (1961) }\end{array}$ \\
\hline 249 & $\mathrm{p}$ & 0.4 & $\mathrm{Fe}^{54}$ & $\sigma_{p, l p l n}$ & $\therefore 20$ & $\begin{array}{l}\text { Radio-Chemical } \\
\text { Techniques }\end{array}$ & $\begin{array}{l}\text { Brookhaven, } \\
\text { Cosmotron; Nevis } \\
\text { Symchrocyclotron, } \\
\text { Columbia }\end{array}$ & $\begin{array}{l}\text { S.s. Markowitz, F.s. Rowland, } \\
\text { and G. Friedlander, Phys. } \\
\text { Rev. 112, } 1295 \text { (1958) }\end{array}$ \\
\hline 250 & $\mathrm{p}$ & 0.500 & $\mathrm{Fe}$ & $\begin{array}{l}\text { Radio-chemical } \\
\text { cross sections }\end{array}$ & 8 & $\begin{array}{l}\text { Radio-Chemical. } \\
\text { Techniques }\end{array}$ & $\begin{array}{l}\text { Berkeley, } \\
\text { Cyclotron }\end{array}$ & $\begin{array}{l}\text { M. Honda and D. Lal, } \\
\text { Phys. Rev. 118, } 1618 \\
\text { (1960) }\end{array}$ \\
\hline 251 & $\mathrm{p}$ & 0.700 & $\mathrm{Fe}$ & $\begin{array}{l}\text { Radio-chemical } \\
\text { cross sections }\end{array}$ & 8 & $\begin{array}{l}\text { Radio-Chernical } \\
\text { Techniques }\end{array}$ & $\begin{array}{l}\text { Berkeley, } \\
\text { Cyclotron }\end{array}$ & $\begin{array}{l}\text { M. Honda and D. Lal, Phys. } \\
\text { Rev. 118, } 1618(1960)\end{array}$ \\
\hline 252 & $\mathrm{p}$ & 2.8 & $\mathrm{Fe}$ & $\sigma_{p X}$ & 5 & $\begin{array}{l}\text { Cloud } \\
\text { Chamber }\end{array}$ & $\begin{array}{l}\text { Brookhaven, } \\
\text { Cosmotron }\end{array}$ & $\begin{array}{l}\text { T. Bowen et al.', Nuovo } \\
\text { cimento } 2,908 \text { (1958) }\end{array}$ \\
\hline 253 & $\mathrm{p}$ & 3 & $\mathrm{Fe}^{54}$ & $\sigma_{p, I p \text { in }}$ & $\sim 20$ & $\begin{array}{l}\text { Radio-Chemical } \\
\text { Techniques }\end{array}$ & $\begin{array}{l}\text { Brookhaven, } \\
\text { Cosmotron; Nevis } \\
\text { Symchrocyclotron, } \\
\text { Columbia }\end{array}$ & $\begin{array}{l}\text { S.s. Markowitz, F.S. Rowland, } \\
\text { and G. Friedlander, Phys. } \\
\text { Rev. 112, } 1295 \text { (1958) }\end{array}$ \\
\hline
\end{tabular}




\begin{tabular}{|c|c|c|c|c|c|c|c|c|c|}
\hline No. & $\begin{array}{l}\text { Incident } \\
\text { Farticle }\end{array}$ & $\begin{array}{c}\text { Incident } \\
\text { Particle } \\
\text { Energy ('Gev) }\end{array}$ & Target & Measured Quantity & $\begin{array}{l}\text { Estimate of } \\
\text { Experimental } \\
\text { Error }(\%)\end{array}$ & Detector & $\begin{array}{l}\text { Installation and } \\
\text { Accelerstor }\end{array}$ & References & Comments \\
\hline 254 & $\mathrm{p}$ & 0.4 & $\mathrm{NN}_{\mathrm{i}} \mathrm{E \varepsilon}$ & $\sigma_{p, \operatorname{lp} \ln }$ & $\sim 20$ & $\begin{array}{l}\text { Radio-Chemical } \\
\text { Technique }\end{array}$ & $\begin{array}{l}\text { Brookhaven, } \\
\text { Cosmotron; Nevis } \\
\text { Symchrocyclotron, } \\
\text { Columbia }\end{array}$ & \multicolumn{2}{|c|}{$\begin{array}{l}\text { S.S. Markowitz, F.s. Rowland, } \\
\text { End G. Friedlander, Phys. } \\
\text { Rev. 112, } 1295 \text { (1958) }\end{array}$} \\
\hline 255 & $\mathrm{p}$ & 3 & $\mathrm{Ni}{ }^{5 \mathrm{~B}}$ & $\sigma_{p, l p l n}$ & $\sim 20$ & $\begin{array}{l}\text { Radio-Chemical } \\
\text { Techniques }\end{array}$ & $\begin{array}{l}\text { Brockhaven, } \\
\text { Cosmotron; Nevis } \\
\text { Synchrocyclotron, } \\
\text { Columbia }\end{array}$ & $\begin{array}{l}\text { S.S. Markowitz, F.S. } \\
\text { Fowland, and G. } \\
\text { Friedlander, Phys. Rev. } \\
\underline{112} 1295 \text { (1958) }\end{array}$ & \\
\hline 256 & $\mathrm{p}$ & 0.077 & $\mathrm{Cr}$ & $\sigma_{\mathrm{pX}}$ & $\sim 3$ & $\begin{array}{l}\text { Range } \\
\text { Telescope }\end{array}$ & $\begin{array}{l}\text { Harvard, } \\
\text { Cyclotron }\end{array}$ & $\begin{array}{l}\text { F. Goloskie and K. } \\
\text { Strauch, Nuclear Phys. } \\
\text { E9, } 474 \text { (1962) }\end{array}$ & Good geometry \\
\hline 257 & $p$ & $0.040-0.150$ & $\mathrm{Cl}$ & $\begin{array}{l}\text { Neutron evaporation } \\
\text { differential cross } \\
\text { section }\end{array}$ & $\sim 15$ & $\begin{array}{l}\text { Recoil } \\
\text { Protons in } \\
\text { Emulsions }\end{array}$ & $\begin{array}{c}\text { Harwell, } \\
\text { Synchrocyclotron }\end{array}$ & $\begin{array}{l}\text { D. Skyrme, Nuclear Phys. } \\
25,177 \text { (1962) }\end{array}$ & \\
\hline 259 & $\mathrm{p}$ & 0.096 & $\mathrm{Cu}$ & $\sigma_{p, x p y}\left(E_{p}, 40^{\circ}\right)$ & 5 & $\begin{array}{l}\text { Range } \\
\text { Telescore }\end{array}$ & $\begin{array}{l}\text { Harvard, } \\
\text { Cyclotron }\end{array}$ & $\begin{array}{l}\text { K. Strauch and F. Titus, } \\
\text { Phys. Rev. 104, igl (1956) }\end{array}$ & \\
\hline 260 & $p$ & 0.113 & $\mathrm{Cu}$ & $\sigma_{p \mathrm{X}}$ & $\sim 3$ & $\begin{array}{l}\text { Range } \\
\text { Telescofe }\end{array}$ & $\begin{array}{l}\text { Harvard, } \\
\text { Cyclotron }\end{array}$ & $\begin{array}{l}\text { R. Goloskie and K. } \\
\text { Strauch, Nuclear Phys. } \\
\text { 29, } 474 \text { (1962) }\end{array}$ & Good geometry \\
\hline 261 & $\mathrm{p}$ & 0.120 & $\mathrm{Cu}$ & $\begin{array}{l}\text { Cross section for } \\
\text { production of } \mathrm{Na}^{24} \\
\text { and } \mathrm{P}^{32}\end{array}$ & - & $\begin{array}{l}\text { Redio-Cremical } \\
\text { Methods }\end{array}$ & $\begin{array}{l}\text { Institute for } \\
\text { Nuclear Problems, } \\
\text { Synchrocyclotron }\end{array}$ & $\begin{array}{l}\text { A.K. Lavrukhina et al., } \\
\text { Atomic Energy } 2,10 \overline{87} \\
\text { (1957) }\end{array}$ & \\
\hline
\end{tabular}




\begin{tabular}{|c|c|c|c|c|c|c|c|c|c|}
\hline No. & $\begin{array}{l}\text { Incident } \\
\text { Particle }\end{array}$ & $\begin{array}{l}\text { Incident } \\
\text { Particle } \\
\text { Energy (Gev) }\end{array}$ & Target & Measured Quantity & $\begin{array}{l}\text { Estimate of } \\
\text { Experimental } \\
\text { Error }(\phi)\end{array}$ & Detector & $\begin{array}{l}\text { Installation and } \\
\text { Accelerator }\end{array}$ & References & Comments \\
\hline 262 & $\mathrm{p}$ & 0.133 & $\mathrm{Cu}$ & $\sigma_{p X}$ & $\sim 3$ & $\begin{array}{l}\text { Range } \\
\text { Telescope }\end{array}$ & $\begin{array}{l}\text { Harvard, } \\
\text { Cyclotron }\end{array}$ & $\begin{array}{l}\text { R. Goloskie and K. } \\
\text { Strauch, Nuclear Phys. } \\
29,474 \text { (1962) }\end{array}$ & Good geometry \\
\hline 263 & $\mathrm{p}$ & 0.143 & $\mathrm{Cu}$ & $\begin{array}{l}\text { Neutron spectra at } \\
\text { zero degrees }\end{array}$ & $3-10$ & $\begin{array}{l}\text { Neutron Time- } \\
\text { of-Flight } \\
\text { Spectrometer }\end{array}$ & $\begin{array}{c}\text { Harwell, } \\
\text { Synchrocyclotron }\end{array}$ & $\begin{array}{l}\text { P.H. Bowen et al., } \\
\text { Nuclear Phys. } 30 \text {, } \\
475 \text { (1962) }\end{array}$ & \\
\hline 264 & $\mathrm{p}$ & 0.220 & $\mathrm{Cu}$ & $\begin{array}{l}\text { Cross section for } \\
\text { production of } \mathrm{Na}^{24} \\
\text { and } \mathrm{P}^{32}\end{array}$ & - & $\begin{array}{l}\text { Radio-Chemical } \\
\text { Methods }\end{array}$ & $\begin{array}{l}\text { Institute for } \\
\text { Nuclear Problems, } \\
\text { Synchrocyclotron }\end{array}$ & $\begin{array}{l}\text { A.K. Lavrukhina et al., } \\
\text { Atomic Energy } 3, \frac{1087}{1057)}\end{array}$ & \\
\hline 265 & $p$ & $0.073-0.400$ & $\mathrm{Cu}^{65}$ & $\begin{array}{l}\text { Recoil energy and } \\
\text { fractions of recoils } \\
\text { forward, backward, } \\
\text { and perpendicular }\end{array}$ & s & $\begin{array}{l}\text { Radio-Chemical } \\
\text { Techniques }\end{array}$ & $\begin{array}{l}\text { Carnegie } \\
\text { Institute, } \\
\text { Synchrocyclotron }\end{array}$ & $\begin{array}{l}\text { E. Mertz and A. Caretto, } \\
\text { Jr., Phys. Rev. 126, } \\
1173 \text { (1962) }\end{array}$ & \\
\hline 266 & $\mathrm{p}$ & $0.082-0.426$ & $\mathrm{Cu}^{65}$ & $\sigma_{p, \operatorname{lp} l n}$ & $2-10$ & $\begin{array}{l}\text { Radio-Chemical } \\
\text { Techniques }\end{array}$ & $\begin{array}{l}\text { Chicago, } \\
\text { Synchrocyclotron }\end{array}$ & $\begin{array}{l}\text { H.P. Yule and A. Turkevich, } \\
\text { Phys. Rev. 118, } 1591 \text { (1960) }\end{array}$ & \\
\hline 267 & $\mathrm{p}$ & 0.340 & $\mathrm{Cu}$ & Neutron yield & 10 & $\begin{array}{l}\mathrm{MnSO}_{4} \\
\text { Solution }\end{array}$ & $\begin{array}{l}\text { Berkeley and } \\
\text { Chicago Cyclotrons }\end{array}$ & $\begin{array}{l}\text { W.E. Crandall and G.P. } \\
\text { Millburn, J. Appl. Phys. } \\
29 \text {. } 698 \text { (1958) }\end{array}$ & \\
\hline 262 & $\mathrm{p}$ & 0.340 & $\mathrm{Cu}$ & $\begin{array}{l}\text { Cross section for } \\
\text { production of } \mathrm{Na}^{24} \\
\text { and } \mathrm{P}^{32}\end{array}$ & - & $\begin{array}{l}\text { Radio-Chemical } \\
\text { Methods }\end{array}$ & $\begin{array}{l}\text {-Institute for } \\
\text { Nuclear Problems, } \\
\text { Synchrocyclotron }\end{array}$ & $\begin{array}{l}\text { A.K. Lavrukhina et al., } \\
\text { Atomic Energy } 3, \frac{\text { al }}{1087} \\
\text { (1957) }\end{array}$ & \\
\hline 269 & $\mathrm{p}$ & 0.4 & $\mathrm{Cu}^{63}$ & $\sigma_{p, l p l n}$ & $\sim 20$ & $\begin{array}{l}\text { Radio-Chemical } \\
\text { Technique }\end{array}$ & $\begin{array}{l}\text { Brookhaven, } \\
\text { Cosmotron; Nevis } \\
\text { Symchrocyclotron, } \\
\text { Columbia }\end{array}$ & $\begin{array}{l}\text { S.S. Markowitz, F.S. } \\
\text { Rowland, and G. Fried- } \\
\text { lander, Phys. Rev. 112, } \\
1295 \text { (1958) }\end{array}$ & \\
\hline
\end{tabular}




\begin{tabular}{|c|c|c|c|c|c|c|c|c|c|}
\hline No. & $\begin{array}{l}\text { Incident } \\
\text { Particle }\end{array}$ & $\begin{array}{l}\text { Incident } \\
\text { Particle } \\
\text { Energy (Gev) }\end{array}$ & Target & Measured Quantity & $\begin{array}{l}\text { Estimate of } \\
\text { Experimental } \\
\text { Error }(\phi)\end{array}$ & Detectar & $\begin{array}{l}\text { Installation and } \\
\text { Accelerator }\end{array}$ & References & Corments \\
\hline 270 & $\mathrm{p}$ & 0.4 & $\mathrm{Cu}^{65}$ & $\sigma_{p, I p l n}$ & $\sim 20$ & $\begin{array}{l}\text { Radio-Chemical } \\
\text { Techniques }\end{array}$ & $\begin{array}{l}\text { Brookhaven, } \\
\text { Cosmotron; Nevis } \\
\text { Symchrocyclotron, } \\
\text { Columbia }\end{array}$ & $\begin{array}{l}\text { S.S. Markovitz, F.S. Row- } \\
\text { land, and G. Friedlander, } \\
\text { Phys. Rev. 1ㅡ, } 1295 \text { (1958) }\end{array}$ & \\
\hline 271 & $\mathrm{p}$ & 0.450 & $\mathrm{Cu}$ & $\begin{array}{c}\sigma_{p, x \pi^{+}}\left(E_{\pi^{+}}, \psi_{\pi^{+}}\right) \\
\sigma_{p}, x \pi^{-} y\left(E_{\pi^{-}}, \psi_{\pi^{-}}\right) \\
\text {with } \psi_{\pi^{ \pm}}=21.5^{\circ}, 60^{\circ}\end{array}$ & $2-40$ & $\begin{array}{l}\text { Counter } \\
\text { Telescope }\end{array}$ & $\begin{array}{c}\text { Chicago, } \\
\text { Synchrocyclotron }\end{array}$ & $\begin{array}{l}\text { E. Lillethun, Phys. Rev. } \\
\underline{125} 665 \text { (1962) }\end{array}$ & Good geometry \\
\hline 272 & $p$ & 0.480 & $\mathrm{Cu}$ & $\begin{array}{l}\text { Cross section for } \\
\text { production of } \mathrm{Na}^{24} \\
\text { and } \mathrm{P}^{32}\end{array}$ & - & $\begin{array}{l}\text { Radio-Chemical } \\
\text { Methods }\end{array}$ & $\begin{array}{l}\text { Institute for } \\
\text { Nuclear Problems, } \\
\text { Synchrocyclotron }\end{array}$ & $\begin{array}{l}\text { A.K. Lavrufhina } \\
\text { Atomic Energy } 3, \frac{\text { et }}{1087} \text { al, } \\
\text { (1957) }\end{array}$ & \\
\hline 273 & $\mathrm{p}$ & $0.185-0.870$ & $\mathrm{Cu}$ & $\sigma_{p, x}$ & 10 & - & - & $\begin{array}{l}\text { G.P. Millburn et al., } \\
\text { Phys. Rev. 95, } 1268 \text {. } \\
(1954)\end{array}$ & A compilation \\
\hline 274 & $\mathrm{p}$ & 0.650 & $\mathrm{Cu}$ & $\begin{array}{l}\sigma_{\mathrm{pT}} \\
\sigma_{\mathrm{pX}}\end{array}$ & 5 & Telescope & $\begin{array}{l}\text { Institute for } \\
\text { Nuclear Problems, } \\
\text { Synchrocyclotron }\end{array}$ & $\begin{array}{l}\text { V.I. Moskalev and B.V. } \\
\text { Gavrilovskii, Soviet } \\
\text { Pnys. Dokledy } 1 \text {, } 607 \\
\text { (1956) }\end{array}$ & $\begin{array}{l}\text { Good and poor } \\
\text { geometry }\end{array}$ \\
\hline 275 & $\mathrm{p}$ & 0.660 & $\begin{array}{l}\mathrm{Cu} \\
\text { with }\end{array}$ & $\begin{array}{c}\sigma_{p, x p y}\left(E_{p}, \psi_{p}\right) \\
\text { for } E_{p} \geq 60 \mathrm{Mev} \\
\psi_{p}=7^{\circ}, 12^{\circ}, 18^{\circ}, 24^{\circ},\end{array}$ & 3 & $\begin{array}{l}\text { Magnetic } \\
\text { Analyzer }\end{array}$ & $\begin{array}{l}\text { Joint Institute } \\
\text { for Nuclear } \\
\text { Research, } \\
\text { Synchrocyclotron }\end{array}$ & $\begin{array}{l}\text { L.S. Azhgirei et al.', } \\
\text { Nuclear Phys. } \frac{13}{258} \\
\text { (1959) }\end{array}$ & \\
\hline 276 & $\mathrm{p}$ & 0.660 & $\mathrm{C} \cdot 2$ & $\begin{array}{l}\text { Cross section for } \\
\text { production of } \mathrm{Na}^{24} \\
\text { and } \mathrm{P}^{32}\end{array}$ & - & $\begin{array}{l}\text { Radio-Chemical } \\
\text { Methods }\end{array}$ & $\begin{array}{l}\text { Institute for } \\
\text { Nuclear Problems, } \\
\text { Synchrocyclotron }\end{array}$ & $\begin{array}{l}\text { A.K. Lavrukhina et al., } \\
\text { Atomic Energy } 3, \frac{10}{107} \\
\text { (1957) }\end{array}$ & \\
\hline 277 & $\mathrm{p}$ & 0.660 & $\mathrm{C}_{12}$ & $\sigma_{p, 1 \pi^{-}}$ & - & $\begin{array}{l}\text { Radio-Chenical } \\
\text { Methods }\end{array}$ & $\begin{array}{l}\text { Institute of Geo- } \\
\text { shemistry and } \\
\text { Analytic Chemistry, } \\
\text { USSR }\end{array}$ & $\begin{array}{l}\text { A.K. Lavrukhina, Soviet } \\
\text { Phys. Doklady } 4,1317 \\
\text { (1959) }\end{array}$ & . \\
\hline
\end{tabular}




\begin{tabular}{|c|c|c|c|c|c|c|c|c|c|}
\hline No. & $\begin{array}{l}\text { Incident } \\
\text { Particle }\end{array}$ & $\begin{array}{l}\text { Incident } \\
\text { Particle. } \\
\text { Energy (Gev) }\end{array}$ & Target & Measured Quantity & $\begin{array}{l}\text { Estimate of } \\
\text { Experimental } \\
\text { Error }(\phi)\end{array}$ & Detector & $\begin{array}{l}\text { Installation and } \\
\text { Accelerator }\end{array}$ & References & Comments \\
\hline 278 & $\mathrm{p}$ & $=0.660$ & $\mathrm{Cu}$ & $\sigma_{p, x \pi^{+} y}\left(E_{\pi^{+}}, 45^{\circ}\right)$ & 10 & $\begin{array}{l}\text { Magnetic } \\
\text { Method } \\
\text { Telescope }\end{array}$ & $\begin{array}{l}\text { Institute for } \\
\text { Nuclear Problems, } \\
\text { Synchrocyclotron }\end{array}$ & $\begin{array}{l}\text { A.G. Meshkovskii et al., } \\
\text { Soviet Phys. -JETP } \frac{\text { al }}{4} \text { (1957) }\end{array}$ & \\
\hline 279 & $\mathrm{p}$ & 0.660 & $\mathrm{Cu}$ & $\sigma_{p, x \pi^{-} y}\left(E_{\pi^{-}}-45^{\circ}\right)$ & 20 & - & $\begin{array}{l}\text { Joint Institute } \\
\text { for Nuclear } \\
\text { Research, } \\
\text { Symchrocyclotron }\end{array}$ & $\begin{array}{l}\text { A.G. Meshkovskii, Ia. } \\
\text { Ia. Shalamov, and V.A. } \\
\text { Shebanov, Soviet Phys.- } \\
\text { JETP 6, } 463 \text { (1958) }\end{array}$ & . \\
\hline 280 & $\mathrm{p}$ & 0.900 & $\mathrm{Cu}$ & $\begin{array}{l}\sigma_{p T} \\
\sigma_{p X}\end{array}$ & $5-10$ & Telescope & $\begin{array}{l}\text { Birmingham, } \\
\text { Synchrotron }\end{array}$ & $\begin{array}{l}\text { N.E. Booth et al., Proc. } \\
\text { Phys. Soc. A(London) } \\
\text { 70, 209 (1957) }\end{array}$ & $\begin{array}{l}\text { Good and poor } \\
\text { geometry }\end{array}$ \\
\hline 281 & $p$ & 1.0 & $\mathrm{Cu}$ & Production of $\mathrm{Be}^{7}$ & $\sim 25$ & $\begin{array}{l}\text { Radio-Chemical } \\
\text { Techniques }\end{array}$ & $\begin{array}{l}\text { Brookhaven, } \\
\text { Cosmotron }\end{array}$ & $\begin{array}{l}\text { E. Baker, G. Friedlander, } \\
\text { and J. Hudis, Phys. Rev. } \\
\text { 112, } 1319 \text { (1958) }\end{array}$ & \\
\hline 282 & $\mathrm{p}$ & 1.1 & $\mathrm{Cu}$ & $\sigma_{p X}$ & $10-20$ & - & Armenia Institute & $\begin{array}{l}\text { N.M. Kocharian et al., } \\
\text { Soviet Phys. Doklady } \\
1,209 \text { (1956) }\end{array}$ & \\
\hline 283 & $\mathrm{p}$ & $0.28-2.2$ & $\mathrm{Cu}^{65}$ & $\sigma_{p, 1 p l n}$ & $\sim 20$ & $\begin{array}{l}\text { Radio-Chemical } \\
\text { Techniques }\end{array}$ & $\begin{array}{l}\text { Brookhaven, } \\
\text { Cosmotron; Nevis } \\
\text { Synchrocyclotron, } \\
\text { Columbia }\end{array}$ & $\begin{array}{l}\text { S.S. Markowitz, F.S. Row- } \\
\text { land, and G. Friedlander, } \\
\text { Phys, Rev. } 112 \text {, } 1295 \\
(1958)\end{array}$ & \\
\hline 284 & $\mathrm{p}$ & 1.8 & $\mathrm{Cu}$ & $\sigma_{\mathrm{pX} .}$. & $10-20$ & - & Armenia Institute & $\begin{array}{l}\text { N.M. Kocharian et al., } \\
\text { Soviet Phys. Doklady } \\
\text { 1, } 209 \text { (1956) }\end{array}$ & - \\
\hline 285 & $\mathrm{p}$ & 2.2 & $\mathrm{Cu}$ & Production of $\mathrm{Be}^{7}$ & $\sim 25$ & $\begin{array}{l}\text { Radio-Chemical } \\
\text { Techniques }\end{array}$ & $\begin{array}{l}\text { Brookhaven, } \\
\text { Cosmotron }\end{array}$ & $\begin{array}{l}\text { E. Baker, G. Friedlander, } \\
\text { and J. Hudis, Phys. Rev. } \\
112 \text {, } 1319 \text { (1958) }\end{array}$ & \\
\hline
\end{tabular}




\begin{tabular}{|c|c|c|c|c|c|c|c|c|c|}
\hline No. & $\begin{array}{l}\text { Incident } \\
\text { Particle }\end{array}$ & $\begin{array}{c}\text { Incident } \\
\text { Particle } \\
\text { Energy (Gev) }\end{array}$ & Target & Measured Quantity & $\begin{array}{l}\text { Estimate of } \\
\text { Experimental } \\
\text { Error }(\%)\end{array}$ & Detector & $\begin{array}{l}\text { Installation and } \\
\text { Accelerator }\end{array}$ & References & Comments \\
\hline 286 & $\mathrm{p}$ & 3.0 & $\mathrm{Cu}$ & Production of $\mathrm{Be}^{7}$ & $\sim 25$ & $\begin{array}{l}\text { Radio-Chemical } \\
\text { Techniques }\end{array}$ & $\begin{array}{l}\text { Brookhaven, } \\
\text { Cosmotron }\end{array}$ & $\begin{array}{l}\text { E. Baker, G. Friedlander, } \\
\text { and J. Hudis, Phys. Rev. } \\
\equiv 12,1319 \text { (1958) }\end{array}$ & \\
\hline 287 & $\mathrm{p}$ & $3 \mathrm{Gev} / \mathrm{c}$ & $\mathrm{Cu}$ & $\begin{array}{l}a_{p, p} \\
a_{p X}\end{array}$ & $5-10$ & $\begin{array}{l}\text { Scintillation } \\
\text { Counters }\end{array}$ & $\begin{array}{l}\text { Berkeley, } \\
\text { Bevatron }\end{array}$ & $\begin{array}{l}\text { M.J. Longo and B.J. Moyer, } \\
\text { Phys. Rev. 125, } 701 \\
\text { (1962) }\end{array}$ & $\begin{array}{l}\text { Good and poor } \\
\text { geometry }\end{array}$ \\
\hline 288 & $\mathrm{p}$ & 3 & $\mathrm{Cu}^{\epsilon 3}$ & $\sigma_{p, 1 p \ln }$ & $\sim 20$ & $\begin{array}{l}\text { Radio-Chemical } \\
\text { Techniques }\end{array}$ & $\begin{array}{l}\text { Brookhaven, } \\
\text { Cosmotron; Nevis } \\
\text { Synchrocyclotron, } \\
\text { Columbia }\end{array}$ & $\begin{array}{l}\text { S.S. Markowitz, F.S. Row- } \\
\text { land, and G. Friedlander, } \\
\text { Phys. Rev. 112 } 1295 \\
(1958)\end{array}$ & \\
\hline 289 & $\mathrm{p}$ & 3 & $\mathrm{Cu}^{65}$ & $\sigma_{p, \perp p l n}$ & $\sim 20$ & $\begin{array}{l}\text { Radio-Chemical } \\
\text { Techniques }\end{array}$ & $\begin{array}{l}\text { Brookhaven, } \\
\text { Cosmotron; Nevis } \\
\text { Synchrocyclotron, } \\
\text { Columbia. }\end{array}$ & $\begin{array}{l}\text { S.s. Markowitz, F.s. Row- } \\
\text { land, and G. Friedlander, } \\
\text { Fhys. Rev. 112, } 1295 \\
(1958)\end{array}$ & . \\
\hline 290 & $\mathrm{p}$ & 3.4 & $\mathrm{Cu}$ & $\sigma_{p X}$ & $10-20$ & - & Armenia Institute & $\begin{array}{l}\text { N.M. Kocharian et al., } \\
\text { Soviet Phys, Doklady } \\
\text { I, } 209 \text { (1956) }\end{array}$ & \\
\hline 291 & $\mathrm{p}$ & 12 & $\mathrm{Cu}$ & ${ }_{p x}$ & $10-20$ & - & Armenia Institute & $\begin{array}{l}\text { N.M. Kocharian et al., } \\
\text { Soviet Phys. Dokledy } \\
\text { 1, } 209 \text { (1958) }\end{array}$ & \\
\hline 292 & $\mathrm{p}$ & $24 \mathrm{Gev} / \mathrm{c}$ & $\mathrm{Cu}$ & $\sigma_{p X}$ & 3 & $\begin{array}{l}\text { Freon } \\
\text { Bubble } \\
\text { Chamber }\end{array}$ & $\begin{array}{l}\text { Un. of Fribourg, } \\
\text { Switz,;CERN } \\
\text { Symchrotron }\end{array}$ & $\begin{array}{l}\text { R.N. Peacock et al., } \\
\text { Nuovo cimento } 22 \text {, } \\
1290 \text { (196́1) }\end{array}$ & \\
\hline 293 & $\mathrm{p}$ & 24 & $\mathrm{Cu}$ & $\begin{array}{l}\text { Cross section for } \\
\text { spallation } \\
\text { products }\end{array}$ & $10-50$ & $\begin{array}{l}\text { Radio-Chemical } \\
\text { Techniques }\end{array}$ & $\begin{array}{c}\text { CERN, } \\
\text { Synchrotron }\end{array}$ & $\begin{array}{l}\text { R. Rudstom, E. Bruninx, } \\
\text { and A.C. Pappas, Phys. } \\
\text { Rev. 126, } 1852 \text { (1962) }\end{array}$ & \\
\hline
\end{tabular}




\begin{tabular}{|c|c|c|c|c|c|c|c|c|c|}
\hline No. & $\begin{array}{l}\text { Incident } \\
\text { Particle }\end{array}$ & $\begin{array}{c}\text { Incident } \\
\text { Particle } \\
\text { Energy (Gev) }\end{array}$ & Target & Measured Quantity & $\begin{array}{l}\text { Estimate of } \\
\text { Experimental } \\
\text { Error }(\phi)\end{array}$ & Detector & $\begin{array}{l}\text { Installation and } \\
\text { Accelerator }\end{array}$ & References & Comments \\
\hline 294 & $p$ & $\dot{0.160}$ & $\mathrm{Zn}$ & $\begin{array}{c}\sigma_{p, x p y}\left(E_{p}, \psi_{p}\right) \\
\text { for } E_{p}<23 \mathrm{Mev} \\
\text { ith } \psi_{p}=60^{\circ}, 90^{\circ}, 120^{\circ}\end{array}$ & $10-20$ & $\begin{array}{l}\text { Range } \\
\text { Telescope }\end{array}$ & $\begin{array}{l}\text { Harvard, } \\
\text { Cyclotron }\end{array}$ & $\begin{array}{l}\text { R. Fox and N.F. Ramsey, } \\
\text { Phys. Rev. 125, } 1609 \\
\text { (1962) }\end{array}$ & . \\
\hline 295 & $\mathrm{p}$ & $0.130-0.425$ & $\mathrm{zn}^{68}$ & $\sigma_{p, x p}$ & $5-40$ & $\begin{array}{l}\text { Radio-Chemical } \\
\text { Techniques }\end{array}$ & $\begin{array}{l}\text { Carnegie Institute, } \\
\text { Synchrocyclotron }\end{array}$ & $\begin{array}{l}\text { D.I. Morrison and A.A. } \\
\text { Caretto, Jr., Phys. } \\
\text { Rev. 127, } 1731 \text { (1962) }\end{array}$ & . \\
\hline 296 & p & 0.4 & $\mathrm{Zn}^{64}$ & $\sigma_{p, \operatorname{lp} \ln }$ & $\sim 20$ & $\begin{array}{l}\text { Radio-Chennical } \\
\text { Techniques }\end{array}$ & $\begin{array}{l}\text { Brookhaven, } \\
\text { Cosmotron; Nevis } \\
\text { Synchrocyclotron, } \\
\text { Columbia }\end{array}$ & $\begin{array}{l}\text { S.s. Markowitz, F.s. Row- } \\
\text { land, and G. Friedlander, } \\
\text { Phys. Rev. } 112 \text {, } 1295 \text { (1958) }\end{array}$ & \\
\hline 297 & $\mathrm{p}$ & 3 & $\mathrm{zn}^{64}$ & $\sigma_{p, I p l n}$ & $\sim 20$ & $\begin{array}{l}\text { Radio-Chemical } \\
\text { Techniques }\end{array}$ & $\begin{array}{l}\text { Brookhaven, } \\
\text { Cosmotron; Nevis } \\
\text { Synchrocyclotron, } \\
\text { Columbia }\end{array}$ & $\begin{array}{l}\text { S.S. Markowitz, F.S. Row- } \\
\text { land, and G. Friedlander, } \\
\text { Phys. Rev. 112, } 1295 \\
\text { (1958) }\end{array}$ & - \\
\hline 298 & $\mathrm{p}$ & 0.5 & $\mathrm{Ga}^{69}$ & $\begin{array}{l}\text { Radio-chemical } \\
\text { cross section }\end{array}$ & $10-30$ & $\begin{array}{l}\text { Radio-Chemical } \\
\text { Techniques }\end{array}$ & $\begin{array}{l}\text { Brookhaven, } \\
\text { Cosmotron }\end{array}$ & $\begin{array}{l}\text { N.T. Porile, Phys. Rev. } \\
125 \text {, } 1379 \text { (1962) }\end{array}$ & \\
\hline 299 & $\mathrm{p}$ & 0.5 & $\mathrm{Ga}^{72}$ & $\begin{array}{l}\text { Radio-chemical } \\
\text { cross section }\end{array}$ & $10-30$ & $\begin{array}{l}\text { Radio-Chemical } \\
\text { Techniques }\end{array}$ & $\begin{array}{l}\text { Brookhaven, } \\
\text { Cosmotron }\end{array}$ & $\begin{array}{l}\text { N.T. Porile, Phys. Rev. } \\
\underline{125} 1379 \text { (1962) }\end{array}$ & \\
\hline 300 & $\mathrm{p}$ & 1.5 & $\mathrm{Ga}^{69}$ & $\begin{array}{l}\text { Radio-chemical } \\
\text { cross section }\end{array}$ & $10-30$ & $\begin{array}{l}\text { Radio-Chemical } \\
\text { Techniques }\end{array}$ & $\begin{array}{l}\text { Brookhaven, } \\
\text { Cosmotron . }\end{array}$ & $\begin{array}{l}\text { N.T. Porile, Phys. Rev. } \\
\text { 125, } 1379(1962)\end{array}$ & \\
\hline 301 & $\mathrm{p}$ & 1.5 & $\mathrm{Ga}^{71}$ & $\begin{array}{l}\text { Radio-chemical } \\
\text { cross section }\end{array}$ & $10-30$ & $\begin{array}{l}\text { Radio-Chemical } \\
\text { Techniques }\end{array}$ & $\begin{array}{l}\text { Brookhaven, } \\
\text { Cosmotron }\end{array}$ & $\begin{array}{l}\text { N.T. Porile, Phys. Rev. } \\
\underline{125} \text {, } 1379 \text { (1962) }\end{array}$ & \\
\hline 302 & $\mathrm{p}$ & 2.9 & $\mathrm{Ga}^{69}$ & $\begin{array}{l}\text { Radio-chemical } \\
\text { cross section }\end{array}$ & $10-30$ & $\begin{array}{l}\text { Radio-Chemical } \\
\text { Techniques }\end{array}$ & $\begin{array}{l}\text { Brookhaven, } \\
\text { Cosmotron }\end{array}$ & $\begin{array}{l}\text { N.T. Porile, Phys. Rev. } \\
125,1379 \text { (1962) }\end{array}$ & \\
\hline
\end{tabular}




\begin{tabular}{|c|c|c|c|c|c|c|c|c|c|}
\hline No. & $\begin{array}{l}\text { Incident } \\
\text { Particle }\end{array}$ & $\begin{array}{l}\text { Incident } \\
\text { Particle } \\
\text { Energy (Gev) }\end{array}$ & Targe & Measured Quantity & $\begin{array}{l}\text { Estimate of } \\
\text { Experimental } \\
\text { Error }(\%)\end{array}$ & Detector & $\begin{array}{l}\text { Installation and } \\
\text { Accelerator }\end{array}$ & References & Comments \\
\hline 303 & $\mathrm{p}$ & 2.9 & $\mathrm{Ga}^{72}$ & $\begin{array}{l}\text { Radio-chemical } \\
\text { cross section }\end{array}$ & $10-30$ & $\begin{array}{l}\text { Radio-Chemical } \\
\text { Techniques }\end{array}$ & $\begin{array}{l}\text { Brookhaven, } \\
\text { Cosmotron }\end{array}$ & $\begin{array}{l}\text { N.T. Porile, Phys. Rev. } \\
\underline{125}, 1379 \text { (1962) }\end{array}$ & \\
\hline 304 & $p$ & $0.130-0.425$ & $\mathrm{As}^{75}$ & $\sigma_{p, x p}$ & $5-40$ & $\begin{array}{l}\text { Radio-Chemical } \\
\text { Techniques }\end{array}$ & $\begin{array}{l}\text { Carnegie Institute, } \\
\text { Synchrocyclotron }\end{array}$ & 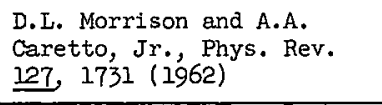 & - \\
\hline 305 & $\mathrm{p}$ & 2.9 & $\mathrm{As}^{75}$ & $\begin{array}{l}A=72, \text { isobaric } \\
\text { cross section }\end{array}$ & $\sim 10$ & $\begin{array}{l}\text { Radio-Chemical } \\
\text { Techniques }\end{array}$ & $\begin{array}{l}\text { Brookhaven, } \\
\text { Cosmotron }\end{array}$ & $\begin{array}{l}\text { S. Kaufman, Phys. Rev. } \\
126,1189 \text { (1962) }\end{array}$ & \\
\hline 306 & $p$ & $0.020-0.150$ & $Y$ & $\sigma_{p, l p l n}$ & 30 & $\begin{array}{l}\text { Radio-Cr.emical } \\
\text { Techniques }\end{array}$ & $\begin{array}{c}\text { Orsay, } \\
\text { Synchrocyclotron }\end{array}$ & $\begin{array}{l}\text { N. Gusakow, Ann. phys. } \\
\text { (French) I, } 67 \text { (1962) }\end{array}$ & \\
\hline 307 & $p$ & 0.060 & $\mathrm{zr}^{90}$ & $\begin{array}{l}\sigma_{p, 2 p 6 n} \\
\sigma_{p, 3 p 5 n}\end{array}$ & 40 & $\begin{array}{l}\text { Radio-Chemical. } \\
\text { Techniques }\end{array}$ & $\begin{array}{l}\text { Rochester, } \\
\text { Synchrocyclotron }\end{array}$ & $\begin{array}{l}\text { E. Ünseren and E.0. Wiig, } \\
\text { Fhys. Rev. 122, } 1875 \\
\text { (1961) }\end{array}$ & \\
\hline 308 & $p$ & 0.100 & $\mathrm{zr} 90$ & $\begin{array}{l}\sigma_{p}, 2 p 6 n \\
\sigma_{p}, 3 p 5 n\end{array}$ & 40 & $\begin{array}{l}\text { Radio-Chemical } \\
\text { Techniques }\end{array}$ & $\begin{array}{l}\because \text { Rochester, } \\
\text { Synchrocyclotron }\end{array}$ & $\begin{array}{l}\text { E. Ünseren and E.0, Wiig, } \\
\text { Fhys. Rev. 122, } 1875, \\
\text { (ig6i) }\end{array}$ & $\because \because$ \\
\hline 309 & $\mathrm{p}$ & 0.150 & $\mathrm{zr}^{90}$ & $\begin{array}{l}\sigma_{p, 2 p 6 n} \\
\sigma_{p, 3 p 5 n}\end{array}$ & 40 & $\begin{array}{l}\text { Radio-Chemical } \\
\text { Techniques }\end{array}$ & $\begin{array}{l}\text { Rochester, } \\
\text { Synchrocyclotron }\end{array}$ & $\begin{array}{l}\text { B. Ünseren and E.0. Wiig, } \\
\text { Fhys. Rev. 122, } 1875 \\
\text { (196i) }\end{array}$ & \\
\hline 310 & $p$ & 0.240 & $2 r^{\infty 0}$ & $\begin{array}{l}\sigma_{p}, 2 p 6 n \\
\sigma_{p}, 3 p 5 n\end{array}$ & 40 & $\begin{array}{l}\text { Redio-Chemical } \\
\text { Techniques }\end{array}$ & $\begin{array}{l}\text { Rochester, } \\
\text { Synchrocyclotron }\end{array}$ & $\begin{array}{l}\text { E. Ünseren and E.O. Wiig, } \\
\text { Phys. Rev. 122, } 1875 \\
\text { (1961) }\end{array}$ & \\
\hline 311 & $\mathrm{p}$ & 0.150 & No. & $\begin{array}{l}\text { Tritium production } \\
\text { cross section }\end{array}$ & $\sim 10$ & $\begin{array}{l}\text { Radio-Chernical } \\
\text { Methods }\end{array}$ & $\begin{array}{c}\text { Orsay, } \\
\text { Synchrocyclotron }\end{array}$ & $\begin{array}{l}\text { C. Brun, M. Lefort, and } \\
\text { צ. Tarrago, J. phys. } \\
\text { radium 23, } 167 \text { (1962) }\end{array}$ & \\
\hline
\end{tabular}




\begin{tabular}{|c|c|c|c|c|c|c|c|c|c|}
\hline No. & $\begin{array}{l}\text { Incident } \\
\text { Particle }\end{array}$ & $\begin{array}{c}\text { Incident } \\
\text { Particle } \\
\text { Energy (Gev) }\end{array}$ & Tariget & Measured Quantity & $\begin{array}{l}\text { Estimate of } \\
\text { Experimental } \\
\text { Error }(\%)\end{array}$ & Detector. & $\begin{array}{l}\text { Installation and } \\
\text { Accelerator }\end{array}$ & References & Comments \\
\hline 312 & $\mathrm{p}$ & 0.4 & Mo' 200 & $\sigma_{p, I p l n}$ & $\sim 20$ & $\begin{array}{l}\text { Radio-Chemical } \\
\text { Techniques }\end{array}$ & $\begin{array}{l}\text { Brookhaven, } \\
\text { Cosmotron; Nevis } \\
\text { Symchrocyclotron, } \\
\text { Columbia }\end{array}$ & $\begin{array}{l}\text { S.s. Markowitz, F.s. Row- } \\
\text { land, and G. Friedlander, } \\
\text { Phys. Rev. 112, } 1295 \\
\text { (1958) }\end{array}$ & \\
\hline 313 & $\mathrm{p}$ & $0.28-2.2$ & $\mathrm{MO}^{100}$ & 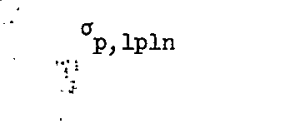 & $\sim 20$ & $\begin{array}{l}\text { Radio-Chemical } \\
\text { Techniques }\end{array}$ & $\begin{array}{l}\text { Brookhaven, } \\
\text { Cosmotron; Nevis } \\
\text { Synchrocyclotron, } \\
\text { Columbia }\end{array}$ & $\begin{array}{l}\text { S.S. Markowitz, F.S. Row- } \\
\text { land, and G. Friedlander, } \\
\text { Phys. Rev. 112, } 1295 \\
\text { (1958) }\end{array}$ & \\
\hline 314 & $\mathrm{p}$ & 3 & $M 0^{100}$ & $\sigma_{p, l p l n}$ & $\sim 20$ & $\begin{array}{l}\text { Radio-Chemical } \\
\text { Techniques }\end{array}$ & $\begin{array}{l}\text { Brookhaven, } \\
\text { Cosmotron; Nevis } \\
\text { Synchrocyclotron, } \\
\text { Columbia }\end{array}$ & $\begin{array}{l}\text { S.S. Markowitz, F.S. Row- } \\
\text { land, and G. Friedlander, } \\
\text { Phys. Rev. 112, } 1295 \\
\text { (1958) }\end{array}$ & \\
\hline 315 & $\mathrm{p}$ & .0 .077 & $A_{G}$ & $\sigma_{p X}$ & $\sim 3$ & $\begin{array}{l}\text { Range } \\
\text { Telescope }\end{array}$ & $\begin{array}{l}\text { Harvard, } \\
\text { Cyclotron }\end{array}$ & $\begin{array}{l}\text { R. Goloskie and K. } \\
\text { Strauch, Nuclear Phys. } \\
\text { 29, } 474 \text { (1962) }\end{array}$ & Good geometry \\
\hline 316 & $\mathrm{p}$ & 0.095 & $A_{E}$ & $\sigma_{p X}$ & $\sim 3$ & $\begin{array}{l}\text { Range } \\
\text { Telescope }\end{array}$ & $\begin{array}{l}\text { Harvard, } \\
\text { Cyclotron }\end{array}$ & $\begin{array}{l}\text { R. Goloskie and K. } \\
\text { Strauch, Nuclear Phys. } \\
\text { 29, } 474 \text { (1962) }\end{array}$ & Good geometry \\
\hline 317 & $\mathrm{p}$ & 0.096 & $A_{G}$ & $\sigma_{p, x p y}\left(E_{p}, 40^{\circ}\right)$ & 5 & $\begin{array}{l}\text { Range } \\
\text { Telescope }\end{array}$ & $\begin{array}{l}\text { Harvard, } \\
\text { Cyclotron }\end{array}$ & $\begin{array}{l}\text { K. Strauch and F. Titus, } \\
\text { Phys. Rev. 104, } 191 \text { (1956) }\end{array}$ & \\
\hline 318 & $\mathrm{p}$ & 0.113 & $\mathrm{Ag}$ & $\sigma_{p X}$ & $\sim 3$ & $\begin{array}{l}\text { Range } \\
\text { Telescope }\end{array}$ & $\begin{array}{l}\text { Harvard, } \\
\text { Cyclotron }\end{array}$ & $\begin{array}{l}\text { R. Goloskie and K. } \\
\text { Strauch, Nuclear Phys. } \\
\text { 29, } 474 \text { (1962) }\end{array}$ & Good geometry \\
\hline 319 & $\mathrm{p}$ & 0.133 & $A g$ & $\sigma_{p X}$ & $\sim 3$ & $\begin{array}{l}\text { Range } \\
\text { Telescope }\end{array}$ & $\begin{array}{l}\text { Harvard, } \\
\text { Cyclotron }\end{array}$ & $\begin{array}{l}\text { R. Goloskie and K. } \\
\text { Strauch, Nuclear Phys. } \\
\text { 29, } 474 \text { (1962) }\end{array}$ & Good geometry \\
\hline
\end{tabular}




\begin{tabular}{|c|c|c|c|c|c|c|c|c|c|}
\hline No. & $\begin{array}{l}\text { Incident } \\
\text { Particle }\end{array}$ & $\begin{array}{l}\text { Incident } \\
\text { Farticle } \\
\text { Energy (Gev) }\end{array}$ & Target & Measured Quantity & $\begin{array}{l}\text { Estimate of } \\
\text { Experimental } \\
\text { Error }(\%)\end{array}$ & Detector & $\begin{array}{c}\text { Installation and } \\
\text { Accelerator }\end{array}$ & References & Comments \\
\hline 320 & $\mathrm{p}$ & 0.150 & Leg & $\begin{array}{l}\text { Energy distribution } \\
\text { of neutrons emitted } \\
\text { in backward direction }\end{array}$ & $\sim 15$ & $\begin{array}{l}\text { Recoil } \\
\text { Protons in } \\
\text { Enulsions }\end{array}$ & $\begin{array}{c}\text { Harwell, } \\
\text { Synchrocyclotron }\end{array}$ & $\begin{array}{l}\text { D. Skyrme, Nuclear } \\
\text { Phys. } 25,177 \text { (1962) }\end{array}$ & \\
\hline 321 & $\mathrm{p}$ & 0.660 & $P_{g}$ & $\begin{array}{l}\sigma_{p, x \pi+y}\left(E_{\pi+}, \psi_{\pi+}\right) \\
\text { for } E_{\pi^{+}}=158 \mathrm{Mev}\end{array}$ & 10 & $\begin{array}{l}\text { Magnet:c } \\
\text { Method } \\
\text { Telescope }\end{array}$ & $\begin{array}{l}\text { Institute for } \\
\text { Nuclear Problems, } \\
\text { Synchrocyclotron }\end{array}$ & $\begin{array}{l}\text { A.G. Meshiovskii et al., } \\
\text { Soviet Phys. -JETP } \frac{1}{4} \text {, } \\
842 \text { (1957) }\end{array}$ & \\
\hline 322 & p & 1.0 & $\mathrm{Ag}$ & Production of $\mathrm{Be}^{7}$ & $\sim 25$ & $\begin{array}{l}\text { Radio-Chemical } \\
\text { Techniques }\end{array}$ & $\begin{array}{l}\text { Brookhaven, } \\
\text { Cosmotron }\end{array}$ & $\begin{array}{l}\text { E. Baker, G. Friedlander, } \\
\text { and J. Hudis, Phys. Rev. } \\
112 \text {, } 1319 \text { (1958) }\end{array}$ & \\
\hline 323 & $\mathrm{p}$ & 2.2 & $\mathrm{Ag}$ & Production of $\mathrm{Be}^{7}$ & $\sim 25$ & $\begin{array}{l}\text { Radio-Chemical } \\
\text { Techniçues }\end{array}$ & $\begin{array}{l}\text { Brookhaven, } \\
\text { Cosmotron }\end{array}$ & 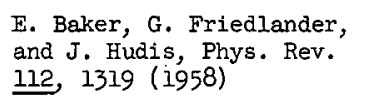 & . \\
\hline 324 & $\mathrm{p}$ & 3.0 & A.s & Production of $\mathrm{Be}^{7}$ & $\sim 25$ & $\begin{array}{l}\text { Radio-Chemical } \\
\text { Techniques }\end{array}$ & $\begin{array}{l}\text { Brookhaven, } \\
\text { Cosmotron }\end{array}$ & $\begin{array}{l}\text { E. Baker, G. Friedlander, } \\
\text { and J. Hudis, Phys. Rev. } \\
112 \text { i319 (1958) }\end{array}$ & \\
\hline 325 & $\mathrm{p}$ & 0.340 & $\mathrm{Ca}$ & Neutron yield & 10 & $\begin{array}{l}\mathrm{MnSO}_{4} \\
\text { Solution }\end{array}$ & $\begin{array}{l}\text { Berkeley and } \\
\text { Chicago Cyclotrons }\end{array}$ & $\begin{array}{l}\text { W.E. Cranciall and G.P. } \\
\text { Yillburn, J. Appl. Phys. } \\
\text { 29, } 698 \text { (1958) }\end{array}$ & \\
\hline 326 & $\mathrm{p}$ & $24 \mathrm{cev} / \mathrm{c}$ & $\mathrm{Ca}$ & $\sigma_{p \mathrm{X}}$ & 3 & $\begin{array}{l}\text { Freon } \\
\text { Bubble } \\
\text { Chamber }\end{array}$ & $\begin{array}{l}\text { Un. of Fribourg, } \\
\text { Switz. ;CERN } \\
\text { Synchrotron }\end{array}$ & $\begin{array}{l}\text { R.N. Peaccck et al., } \\
\text { Nuovo cimento } 22 \text {, } \\
1290 \text { (1961) }\end{array}$ & \\
\hline 327 & $\mathrm{p}$ & 0.180 & In & $\sigma_{p X}$ & 3 & $\begin{array}{l}\text { Range } \\
\text { Separator }\end{array}$ & $\begin{array}{l}\text { Upsala, } \\
\text { Cyclotron }\end{array}$ & $\begin{array}{l}\text { A. Johansson, U. Svanberg, } \\
\text { and 0. Sundberg, Arkiv } \\
\text { Fysik 19, } 527 \text { (1961) }\end{array}$ & Poor geometry \\
\hline 328 & $\mathrm{p}$ & 1, & In & $\begin{array}{l}\text { Radio-chemical } \\
\text { cross sections }\end{array}$ & 30. & $\begin{array}{l}\text { Radio-Chemical } \\
\text { Techniques }\end{array}$ & $\begin{array}{l}\text { Berkeley, } \\
\text { Bevatron }\end{array}$ & $\begin{array}{l}\text { D.R. Nethaway and L. } \\
\text { Winsberg, Phys. Rev. } \\
119,137 \bar{\nu}(1960)\end{array}$ & \\
\hline
\end{tabular}




\begin{tabular}{|c|c|c|c|c|c|c|c|c|c|}
\hline No. & $\begin{array}{l}\text { Incident } \\
\text { Particle }\end{array}$ & $\begin{array}{c}\text { Incident } \\
\text { Particle } \\
\text { Energy (Gev) }\end{array}$ & Target & Measured Quantity & $\begin{array}{l}\text { Estimate of } \\
\text { Experimental } \\
\text { Error }(\%)\end{array}$ & Detector & $\begin{array}{l}\text { Installation and } \\
\text { Accelerator }\end{array}$ & References & \\
\hline 329 & $\mathrm{p}$ & 2 & $I n$ & $\begin{array}{l}\text { Radio-chemical } \\
\text { cross sections }\end{array}$ & 30 & $\begin{array}{l}\text { Radio-Chemical } \\
\text { Techniques }\end{array}$ & $\begin{array}{l}\text { Berkeley, } \\
\text { Bevatron }\end{array}$ & $\begin{array}{l}\text { D. R. Nethaway and L. } \\
\text { Winsberg, Phys. Rev. } \\
119 \text {, } 1375 \text { (1960) }\end{array}$ & \\
\hline 330 & $\mathrm{p}$ & 2.9 & In & $\begin{array}{l}\text { Radio-chemical } \\
\text { cross sections }\end{array}$ & - & $\begin{array}{l}\text { Radio-Chemical } \\
\text { Techniques }\end{array}$ & $\begin{array}{l}\text { Brookhaven, } \\
\text { Cosmotron }\end{array}$ & $\begin{array}{l}\text { N. T. Porile, Phys. Rev. } \\
128,1916(1962)\end{array}$ & \\
\hline 331 & $\mathrm{p}$ & $0.25-6.2$ & In & $\begin{array}{l}\text { Radio-chemical } \\
\text { cross sections }\end{array}$ & 25 & $\begin{array}{l}\text { Radio-Chemical } \\
\text { Techniques }\end{array}$ & $\begin{array}{l}\text { Berkeley, } \\
\text { Cyclotron and } \\
\text { Bevatron }\end{array}$ & $\begin{array}{l}\text { I.M. Ladenbauer. and L. } \\
\text { Winsberg, Phys. Rev. } \\
\text { 1ㅡ, } 1368 \text { (1960) }\end{array}$ & \\
\hline 332 & $\mathrm{p}$ & 4.1 & In & $\begin{array}{l}\text { Radio-chemical } \\
\text { cross sections }\end{array}$ & 30 & $\begin{array}{l}\text { Radio-Chemical } \\
\text { Techn_ques }\end{array}$ & $\begin{array}{l}\text { Berkeley, } \\
\text { Bevatron }\end{array}$ & $\begin{array}{l}\text { D.R. Nethaway and L. } \\
\text { Winsberg, Phys. Rev. } \\
\text { 119, } 1375 \text { (1960) }\end{array}$ & . \\
\hline 333 & $\mathrm{p}$ & 6.2 & In & $\begin{array}{l}\text { Radio-chemical } \\
\text { cross sections }\end{array}$ & 30 & $\begin{array}{l}\text { Radio-Chemical } \\
\text { Techniques }\end{array}$ & $\begin{array}{l}\text { Berkeley, } \\
\text { Bevatron }\end{array}$ & $\begin{array}{l}\text { D.R. Nethaway and I. } \\
\text { Winsberg, Phys. Rev. } \\
\text { 119, } 1375 \text { (1960) }\end{array}$ & \\
\hline 334 & $\mathrm{p}$ & 30 & In & $\begin{array}{l}\text { Radio-chemical } \\
\text { cross sections }\end{array}$ & - & $\begin{array}{l}\text { Radio-Chemical } \\
\text { Techniques }\end{array}$ & $\begin{array}{l}\text { Brookhaven, } \\
\text { Synchrotron }\end{array}$ & $\begin{array}{l}\text { N. T. Porile, Phys. Rev. } \\
\underline{128}, 1916(1962)\end{array}$ & \\
\hline 335 & $\mathrm{p}$ & 0.061 & $\mathrm{sn}$ & $\begin{array}{l}\sigma_{p T} \\
\sigma_{p X}\end{array}$ & 6 & $\begin{array}{l}\text { Plastic } \\
\text { Scintillators }\end{array}$ & Minnesota & $\begin{array}{l}\text { v. Meyer, R.M. Eisberg, } \\
\text { and R.F. Carlson, Phys. } \\
\text { Rev. 117, } 1334 \text { (1960) }\end{array}$ & \\
\hline 336 & $\mathrm{p}$ & 0.150 & $\mathrm{Sn}$ & $\begin{array}{l}\text { Tritium production } \\
\text { cross section }\end{array}$ & $\sim 10$ & $\begin{array}{l}\text { Radio-Chemical } \\
\text { Methods }\end{array}$ & $\begin{array}{l}\text { Orsay, } \\
\text { Synchrocyclotron }\end{array}$ & $\begin{array}{l}\text { C. Brun, M. Lefort, and } \\
\text { X. Tarrago, J. phys. } \\
\text { radium 23, } 167 \text { (1962) }\end{array}$ & - \\
\hline
\end{tabular}




\begin{tabular}{|c|c|c|c|c|c|c|c|c|c|}
\hline INo. & $\begin{array}{l}\text { Incident } \\
\text { Particle }\end{array}$ & $\begin{array}{c}\text { Incident } \\
\text { Farticle } \\
\text { Energy (Gev) }\end{array}$ & Target & Measured Quantity & $\begin{array}{l}\text { Estimate of } \\
\text { Experimental } \\
\text { Error }(\phi)\end{array}$ & Detector & $\begin{array}{l}\text { Installation and } \\
\text { Accelerator }\end{array}$ & References & Comments \\
\hline 337 & $\mathrm{p}$ & 0.160 & $\mathrm{Sn}$ & $\begin{array}{c}\sigma_{p}, x p y\left(E_{p}, \psi_{p}\right) \\
\text { for } E_{p}<23 \mathrm{Mev} \\
\text { with } \psi_{p}=60^{\circ}, 90^{\circ}, 120^{\circ}\end{array}$ & $10-20$ & $\begin{array}{l}\text { Range. } \\
\text { Teles }=0 p e\end{array}$ & $\begin{array}{l}\text { Harvard, } \\
\text { Cyclotron }\end{array}$ & $\begin{array}{l}\text { R. Fox and N.F. Ramsey, } \\
\text { Phys. Rev. 125, } 1609 \\
(1962)\end{array}$ & \\
\hline 338 & $\mathrm{p}$ & 0.450 & $\mathrm{Sn}$ & $\begin{array}{c}\sigma_{p, x \pi^{+} y}\left(E_{\pi^{+}}, \psi_{\pi^{+}}\right) \\
\sigma_{p, x \pi^{-}}\left(E_{\pi^{-}}, \psi_{\pi^{-}}\right) \\
\text {with } \psi_{\pi^{ \pm}}=21.5^{\circ}, 60^{\circ}\end{array}$ & $2-40$ & $\begin{array}{l}\text { Counter } \\
\text { Telescope }\end{array}$ & $\begin{array}{l}\text { Chicago, } \\
\text { symchrocyclotron }\end{array}$ & $\begin{array}{l}\text { E. Lillethun, Phys. } \\
\text { Rev. 125, } 665 \text { (1962) }\end{array}$ & Good geometry \\
\hline 339 & $\mathrm{p}$ & $0.300-0.820$ & $\mathrm{Sn}$ & Neutrons produced & $10-20$ & $\begin{array}{l}\text { Paraffin Block } \\
\text { and } \mathrm{B}^{10} \mathrm{~F}_{3} \\
\text { Counters }\end{array}$ & $\begin{array}{l}\text { Chalk River; Expo- } \\
\text { sure Made at } \\
\text { Echo Lake, Cal. }\end{array}$ & $\begin{array}{l}\text { M. Bercovitch et al., Phys. } \\
\text { Rev. } 119,412 \text { (1960) }\end{array}$ & \\
\hline 340 & $\mathrm{p}$ & 0.650 & $\mathrm{Sn}$ & $\begin{array}{l}\sigma_{p T} \\
\sigma_{p X}\end{array}$ & 5 & Telescope & $\begin{array}{l}\text { Institute for } \\
\text { Nuclear Problems, } \\
\text { Synchrocyclotron }\end{array}$ & $\begin{array}{l}\text { V.I. Moskalev and B.v. } \\
\text { Gavrilovskii, Soviet } \\
\text { Phys. Doklady I, } 607 \\
\text { (1956). }\end{array}$ & $\begin{array}{l}\text { Good and poor } \\
\text { geometry }\end{array}$ \\
\hline 341 & $p$ & 0.450 & $\mathrm{Sb}$ & 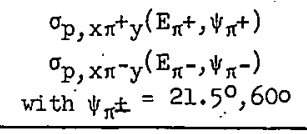 & $2-40$ & $\begin{array}{l}\text { Counter } \\
\text { Telescope }\end{array}$ & $\begin{array}{l}\text { Chicago, } \\
\text { Synchrocyclotron }\end{array}$ & $\begin{array}{l}\text { E. Lillethun, Phys. Rev. } \\
\underline{125}, 665 \text { (1962) }\end{array}$ & Good geometry \\
\hline 342 & $\mathrm{p}$ & 0.900 & $\mathrm{sb}$ & $\begin{array}{l}\sigma_{\mathrm{pT}} \\
\sigma_{\mathrm{pX}} \\
\end{array}$ & $5-10$ & Telescope & $\begin{array}{l}\text { Birmingham, } \\
\text { Synchrotron }\end{array}$ & $\begin{array}{l}\text { NoE. Booth et al., Proc. } \\
\text { Phys. Soc, A(London) i0, } \\
209 \text { (1957) }\end{array}$ & $\begin{array}{l}\text { Good and poor } \\
\text { geometry }\end{array}$ \\
\hline 343 & $\mathrm{p}$ & $0.020-0.150$ & Cs & $\sigma_{p, I p l n}$ & 30 & $\begin{array}{l}\text { Radio-Chemical } \\
\text { Techniques. }\end{array}$ & $\begin{array}{l}\text { Orsay, } \\
\text { Synchrocyclotron }\end{array}$ & $\begin{array}{l}\text { M. Gusakow, Ann. phys. } \\
\text { (French) I, } 67 \text { (1962) }\end{array}$ & \\
\hline 344 & $\mathrm{p}$ & $0.020-0.255$ & Cs & $\sigma_{p, l p I n}$ & $\sim 30$ & $\begin{array}{l}\text { Radio-Chemical } \\
\text { Techniques }\end{array}$ & $\begin{array}{c}\text { Orsay, } \\
\text { Synchrocyclotron }\end{array}$ & $\begin{array}{l}\text { M. Gusakow et al." J. } \\
\text { phys. radium } 22 \text {. } 636 \\
\text { (196i) }\end{array}$ & \\
\hline
\end{tabular}




\begin{tabular}{|c|c|c|c|c|c|c|c|c|c|}
\hline No. & $\begin{array}{l}\text { Incident } \\
\text { Particle }\end{array}$ & $\begin{array}{l}\text { Incident } \\
\text { Particle } \\
\text { Energy (Gev) }\end{array}$ & Target & Measured Quantity & $\begin{array}{l}\text { Estimate of } \\
\text { Experimental } \\
\text { Error }(\phi)\end{array}$ & Detector & $\begin{array}{l}\text { Installation and } \\
\text { Accelerator }\end{array}$ & References & Comments \\
\hline 345 & $\mathrm{p}$ & $0.030-0.155$ & Cs & ${ }^{\sigma} \mathrm{p}, \operatorname{lp} \times \mathrm{n}$ & $\sim 25$ & $\begin{array}{l}\text { Radio-Chemical } \\
\text { Techniques }\end{array}$ & $\begin{array}{c}\text { Orsay, } \\
\text { Synchrocyclotron }\end{array}$ & $\begin{array}{l}\text { C. Riehl, J. phys. } \\
\text { radium } 22,770 \text { (1961) }\end{array}$ & \\
\hline 346 & $\mathrm{p}$ & 0.120 & La & $\begin{array}{l}\text { Cross section for } \\
\text { production of } \mathrm{Na}^{24} \\
\text { and } \mathrm{P}^{32}\end{array}$ & $\therefore$ & $\begin{array}{l}\text { Radio-Chemical } \\
\text { Methods }\end{array}$ & $\begin{array}{l}\text { Institute for } \\
\text { Nuclear Problems, } \\
\text { Synchrocyclotron }\end{array}$ & $\begin{array}{l}\text { A.K. Lavrukhina et } \frac{a 1}{10} \text {, } \\
\text { Atomic Energy } 2, \frac{1087}{\text { (1957) }}\end{array}$ & \\
\hline 347. & $\mathrm{p}$ & $0.220^{\circ}$ & $\mathrm{La}$ & $\begin{array}{l}\text { Cross section for } \\
\text { production of } \mathrm{Na}^{24} \\
\text { and } \mathrm{P}^{32}\end{array}$ & - & $\begin{array}{l}\text { Radio-Chemical } \\
\text { Methods }\end{array}$ & $\begin{array}{l}\text { Institute for } \\
\text { Nuclear Problems, } \\
\text { Synchrocyclotron }\end{array}$ & $\begin{array}{l}\text { A.K. Lavrukhina et al., } \\
\text { Atomic Energy } 3, \frac{1087}{108} \\
\text { (1957) }\end{array}$ & . \\
\hline 348 & $\mathrm{p}$ & 0.340 & $\mathrm{La}$ & $\begin{array}{l}\text { Cross section for } \\
\text { production of } \cdot \mathrm{Na}^{24} \\
\text { and } \mathrm{P}^{32}\end{array}$ & - & $\begin{array}{l}\text { Radio-Chemical } \\
\text { Methods }\end{array}$ & $\begin{array}{l}\text { Institute for } \\
\text { Nuclear Problems, } \\
\text { Synchrocyclotron }\end{array}$ & $\begin{array}{l}\text { A.K. Lavrukhina et al., } \\
\text { Atomic Energy } 2, \frac{1087}{1} \text { (1957) }\end{array}$ & . \\
\hline 349 & $p$ & 0.480 & La: & $\begin{array}{l}\text { Cross section for } \\
\text { production of } \mathrm{Na}^{24} \\
\text { and } \mathrm{P}^{32}\end{array}$ & - & $\begin{array}{l}\text { Radio-Chemical } \\
\text { Methods }\end{array}$ & $\begin{array}{l}\text { Institute for } \\
\text { Nuclear Problems, } \\
\text { Symchrocyclotron }\end{array}$ & $\begin{array}{l}\text { A.K. Lavrukhina et al., } \\
\text { Atomic Energy } 3, \text { 1087 } \\
\text { (1957) }\end{array}$ & \\
\hline 350 & $\mathrm{p}$ & 0.660 & $\mathrm{La}$ & $\begin{array}{l}\text { Cross section for } \\
\text { production of } \mathrm{Na}^{24} \\
\text { and } \mathrm{P}^{32}\end{array}$ & - & $\begin{array}{l}\text { Radio-Chemical } \\
\text { Methods }\end{array}$ & $\begin{array}{l}\text { Insti tute for } \\
\text { Nuclear Problems, } \\
\text { Synchrocyclotron }\end{array}$ & $\begin{array}{l}\text { A.K. Lavrukhina et al., } \\
\text { Atomic Energy } 3, \frac{1087}{1057)} \\
\text { (1957) }\end{array}$ & \\
\hline 351 & $\mathrm{p}$ & $0.060-0.233$ & $\mathrm{Ce}^{242}$ & $\begin{array}{l}\sigma_{p}, 1 p \ln \\
\sigma_{p, 2 p}\end{array}$ & $5-40$ & $\begin{array}{l}\text { Radio-Chemical } \\
\text { Techniques }\end{array}$ & $\begin{array}{c}\text { Rochester, } \\
\text { Synchrocyclotron }\end{array}$ & $\begin{array}{l}\text { W.R. Ware and E.O. Wi.ig, } \\
\text { Phys. Rev. 122, } 1837 \\
\text { (1961) }\end{array}$ & . \\
\hline 352. & $\mathrm{p}$ & 0.4 & $\mathrm{Ce}^{142}$ & $\begin{array}{l}\sigma_{\mathrm{p},}, \mathrm{pln} \\
\sigma_{\mathrm{p}, 2 \mathrm{p}}\end{array}$ & $3-25$ & $\begin{array}{l}\text { Radio-Chemical } \\
\text { Techniques }\end{array}$ & $\begin{array}{l}\text { Brookhaven, } \\
\text { Cosmotron }\end{array}$ & $\begin{array}{l}\text { A.A. Caretto and G. Fried- } \\
\text { lander, Phys. Rev. } 110 \\
1169 \text { (1958) }\end{array}$ & \\
\hline 353 & $\mathrm{p}$ & 0.660 & $\mathrm{Ce}$ & Spallation products & - & $\begin{array}{l}\text { Electrolyte } \\
\text { Deposition }\end{array}$ & $\begin{array}{l}\text { Institute for } \\
\text { Nuclear Research, } \\
\text { Synchrocyclotron }\end{array}$ & $\begin{array}{l}\text { A.K. Lavrukhina, G.M. Kolesov } \\
\text { and Tan Syao-en, Bull. } \\
\text { Acad. Sci. USSR 24, } 1117 \\
\text { (1960) }\end{array}$ & \\
\hline
\end{tabular}




\begin{tabular}{|c|c|c|c|c|c|c|c|c|c|}
\hline No. & $\begin{array}{l}\text { Incident } \\
\text { Particle }\end{array}$ & $\begin{array}{l}\text { Incident } \\
\text { Particle } \\
\text { Energy (Gev) }\end{array}$ & Targe & Measured Quantity & $\begin{array}{l}\text { Estimate of } \\
\text { Experimental } \\
\text { Error }(\%)\end{array}$ & Detectcr & $\begin{array}{l}\text { Installation and } \\
\text { Accelerator }\end{array}$ & References & Comments \\
\hline 354 & $\mathrm{p}$ & 1.0 & $C e^{142}$ & $\begin{array}{l}\sigma_{\mathrm{p}, \mathrm{lp} \perp \mathrm{n}} \\
\sigma_{\mathrm{p}, 2 \mathrm{p}}\end{array}$ & $3-25$ & $\begin{array}{l}\text { Radio-Cremical } \\
\text { Techniques }\end{array}$ & $\begin{array}{l}\text { Brookhaven, } \\
\text { Cosmotron }\end{array}$ & 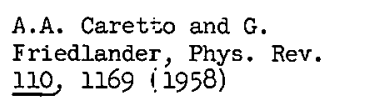 & \\
\hline 355 & $p$ & 2.2 & $\mathrm{Ce}^{142}$ & $\begin{array}{l}\sigma_{p, l p l n} \\
\sigma_{p, 2 p}\end{array}$ & $3-25$ & $\begin{array}{l}\text { Radio-Chemical } \\
\text { Techniques }\end{array}$ & $\begin{array}{l}\text { Brookhaven, } \\
\text { Cosmotron }\end{array}$ & $\begin{array}{l}\text { A. A. Caretio and G. } \\
\text { Priedlander, Phys. Rev. } \\
\text { 110, } 1169: 1958)\end{array}$ & \\
\hline 356 & $p$ & 3.0 & $\mathrm{Ce}^{142}$ & $\begin{array}{l}\sigma_{p, l p l n} \\
\sigma_{p, 2 p}\end{array}$ & $3-25$ & $\begin{array}{l}\text { Radio-Chemical } \\
\text { Techniques }\end{array}$ & $\begin{array}{l}\text { Brookhaven, } \\
\text { Cosmotron }\end{array}$ & $\begin{array}{l}\text { R.A. Caretto and G. } \\
\text { Friedlander, Phys. Rev. } \\
\text { 110, } 1169 \text { (1958) }\end{array}$ & \\
\hline $35 T$ & $\mathrm{p}$ & 0.660 & $\mathrm{He}$ & $\begin{array}{l}\text { Radio-chemical } \\
\text { cross sections }\end{array}$ & - & - & - & $\begin{array}{l}\text { A.K. Labrukhin and A.A. } \\
\text { Pozdnyakov, Atomic } \\
\text { Energy I } 362(1960)\end{array}$ & \\
\hline 358 & $\mathrm{p}$ & c. 160 & Tá & $\begin{array}{c}\sigma_{p, x \supseteq y}\left(E_{p}, \psi_{p}\right) \\
\text { for } E_{p}<23 \mathrm{Mev} \\
c \psi_{p}=60^{\circ}, 90^{\circ}, 120^{\circ}\end{array}$ & $10-20$ & $\begin{array}{l}\text { Range } \\
\text { Telescope }\end{array}$ & $\begin{array}{l}\text { Harvard, } \\
\text { Cyclotron }\end{array}$ & $\begin{array}{l}\text { R. Fox and N.F. Ramsey, } \\
\text { Phys. Rev. 125, } 1609 \\
(1962)\end{array}$ & \\
\hline 359 & $\mathrm{p}$ & 0.4 & $\mathrm{Ta}^{181}$ & $\sigma_{p, 1 p l n}$ & $\sim 20$ & $\begin{array}{l}\text { Radio-Chenical } \\
\text { Techniques }\end{array}$ & $\begin{array}{l}\text { Brookhaven, } \\
\text { Cosmotron; Nevis } \\
\text { Synchrocyclotron, } \\
\text { Columbia }\end{array}$ & $\begin{array}{l}\text { S.s. Markc.witz, F.S. Row- } \\
\text { land, and G. Friedlander, } \\
\text { Phys. Rev. I12, } 1295 \text { (1958) }\end{array}$ & \\
\hline 360 & $\mathrm{p}$ & $0.28-2.2$ & $\mathrm{Ta}^{28 \mathrm{i}}$ & ${ }^{\circ}, \operatorname{lp} \ln$ & $\sim 20$. & $\begin{array}{l}\text { Radio-Chemical } \\
\text { Techniques }\end{array}$ & $\begin{array}{l}\text { Brookhaven, } \\
\text { Cosmotron; Nevis } \\
\text { Synchrocyclotron, } \\
\text { Columbia }\end{array}$ & $\begin{array}{l}\text { S.s. Markowitz, F.s. Row- } \\
\text { land, and G. Friedlander, } \\
\text { Phys. Rev. 112, } 1295 \text { (1958) }\end{array}$ & \\
\hline
\end{tabular}




\begin{tabular}{|c|c|c|c|c|c|c|c|c|c|}
\hline No. & $\begin{array}{l}\text { Incident } \\
\text { Particle }\end{array}$ & $\begin{array}{l}\text { Incicient } \\
\text { Particle } \\
\text { Energy (Gev) }\end{array}$ & Target & Measured Quantity & $\begin{array}{l}\text { Estimate of } \\
\text { Experimental } \\
\text { Error }(\phi)\end{array}$ & Detector & $\begin{array}{l}\text { Installation and } \\
\text { Accelerator }\end{array}$ & References & Comments \\
\hline 361 & $\mathrm{p}$ & 3 & $\mathrm{Ta}^{181}$ & $\sigma_{p}, 2 p \ln$ & $\sim 20$ & $\begin{array}{l}\text { Radio-Chemical } \\
\text { Techniques }\end{array}$ & $\begin{array}{l}\text { Brookhaven, } \\
\text { Cosmotron; Nevis } \\
\text { Synchrocyclotron, } \\
\text { Columbia }\end{array}$ & $\begin{array}{l}\text { S.S. Markowitz, F.S. Row- } \\
\text { land, and G. Friedlander, } \\
\text { Phys. Rev. 112, } 1295 \\
\text { (1958) }\end{array}$ & . \\
\hline 362 & $\mathrm{p}$ & 5.7 & $\mathrm{Ta}$ & $\begin{array}{l}\text { Radio-chemical } \\
\text { cross sections } \\
\text { for } 67 \text { nuclei }\end{array}$ & $15-100$ & $\begin{array}{l}\text { Radio-Chemical } \\
\text { Techniques }\end{array}$ & $\begin{array}{l}\text { Berkeley, } \\
\text { Bevatron }\end{array}$ & $\begin{array}{l}\text { J. Grover, Phys. Rev. } \\
126,1540 \text { (1962) }\end{array}$ & \\
\hline $363^{\circ}$ & $\mathrm{p}$ & $0.040-0.150$ & w & $\begin{array}{l}\text { Neutron evaporation } \\
\text { differential cross } \\
\text { section }\end{array}$ & $\sim 15$ & $\begin{array}{l}\text { Recoil } \\
\text { Protons in } \\
\text { Emulsions }\end{array}$ & $\begin{array}{c}\text { Harwell, } \\
\text { Synchrocyclotron }\end{array}$ & $\begin{array}{l}\text { D. Skyrme, Nuclear Phys. } \\
\text { 35, } 177 \text { (1962) }\end{array}$ & \\
\hline 364 & $\mathrm{p}$ & 0.138 & w & $\begin{array}{l}\text { Angular distribution } \\
\text { of evaporation } \\
\text { neutrons }\end{array}$ & $n \sim 15$ & $\begin{array}{l}\text { Recoil } \\
\text { Protons in } \\
\text { Emulsions }\end{array}$ & $\begin{array}{c}\text { Harwell, } \\
\text { Synchrocyclotron }\end{array}$ & $\begin{array}{l}\text { D. Skyrme, Nuclear Phys. } \\
35,177 \text { (1962) }\end{array}$ & \\
\hline 365 & $\mathrm{p}$ & $0 . \Sigma 51$ & w & $\begin{array}{l}\text { Energy distribution } \\
\text { of neutrons emit- } \\
\text { ted in backward } \\
\text { direction }\end{array}$ & $\sim .15$ & $\begin{array}{l}\text { Recoil } \\
\text { Protons in } \\
\text { Emulsions }\end{array}$ & $\begin{array}{c}\text { Harwell, } \\
\text { Synchrocyclotron }\end{array}$ & $\begin{array}{l}\text { D. Skyrme, Nuclear Phys. } \\
35,177 \text { (1962) }\end{array}$ & ; \\
\hline 366 & $\mathrm{p}$ & $0.130-0.425$ & $W^{180}$ & $\sigma_{p, x p}$ & $5-40$ & $\begin{array}{l}\text { Radio-Chemical } \\
\text { Techniques }\end{array}$ & $\begin{array}{l}\text { Carnegie Institute, } \\
\text { Synchrocyclotron }\end{array}$ & $\begin{array}{l}\text { D.L. Morrison and A.A. } \\
\text { Caretto, Jr., Phys. Rev. } \\
\text { 127, } 1731 \text { (1962) }\end{array}$ & . \\
\hline 367 & $\mathrm{p}$ & $0.300-0.820$ & W & Neutrons produced & $10-20$ & $\begin{array}{l}\text { Paraffin Block, } \\
\mathrm{B}^{10} \mathrm{~F}_{3} \text { Counters }\end{array}$ & $\begin{array}{l}\text { Chalk River; Ex- } \\
\text { posure Made at } \\
\text { Echo Lake, Cal. }\end{array}$ & $\begin{array}{l}\text { M. Bercovitch et al., Phys. } \\
\text { Rev. } \underline{119} 412(1960)\end{array}$ & \\
\hline 368 & $\mathrm{p}$ & $0.130-0.425$ & $\operatorname{Re}=87$ & $\sigma_{p, x p}$ & $5-40$ & $\begin{array}{l}\text { Radio-Chemical } \\
\text { Techniques }\end{array}$ & $\begin{array}{l}\text { Carnegie Institute, } \\
\text { Synchrocyclotron }\end{array}$ & $\begin{array}{l}\text { D. L. Morrison and A.A. } \\
\text { Caretto, Jr., Phys. } \\
\text { Rev. 127, } 1731 \text { (1962) }\end{array}$ & \\
\hline
\end{tabular}




\begin{tabular}{|c|c|c|c|c|c|c|c|c|c|}
\hline No. & $\begin{array}{l}\text { Incident } \\
\text { Particle }\end{array}$ & $\begin{array}{l}\text { Incident } \\
\text { Particle } \\
\text { Energy (Gev) }\end{array}$ & Target & Measured Quantity & $\begin{array}{l}\text { Estimate of } \\
\text { Experimental } \\
\text { Error }(\%)\end{array}$ & Detector & $\begin{array}{l}\text { Installation and } \\
\text { Accelerator }\end{array}$ & References & Conments \\
\hline 369 & $\mathrm{p}$ & $0.0=8-0.086$ & $\mathrm{Au}^{197}$ & $\begin{array}{l}\sigma_{p, I p l n} \\
\sigma_{p, I p 2 n} \\
\sigma_{p, I p 3 n}\end{array}$ & 13 & $\begin{array}{l}\text { Radio-Chemical } \\
\text { Techniques }\end{array}$ & $\begin{array}{l}\text { McGill, } \\
\text { Synchrocyclotron }\end{array}$ & $\begin{array}{l}\text { T.M. Kavanagh and } \\
\text { R.E. Bel1, Can. J. } \\
\text { Phys. 39, } 1172 \text { (1961) }\end{array}$ & \\
\hline 370 & $\mathrm{p}$ & 0.082 & A.d & $\begin{array}{l}\text { Tritium production } \\
\text { cross section }\end{array}$ & $\sim 10$ & $\begin{array}{l}\text { Radio-Chemical } \\
\text { Methods }\end{array}$ & $\begin{array}{c}\text { Orsay, } \\
\text { Synchrocyclotron }\end{array}$ & $\begin{array}{l}\text { C. Brun, M. Lefort, and } \\
\text { x. Tarrago, J. phys. radium } \\
\text { 23, } 167 \text { (1962) }\end{array}$ & \\
\hline 371 & p & $0.020-0.250$ & Au & $\sigma_{p, I p \ln }$ & 30 & $\begin{array}{l}\text { Radio-Chenical } \\
\text { Techniques }\end{array}$ & $\begin{array}{c}\text { Orsay, } \\
\text { Synchrocyclotron }\end{array}$ & $\begin{array}{l}\text { M. Gusakow, Ann, phys. } \\
\text { (French) I, } 67 \text { (1962) }\end{array}$ & \\
\hline 372 & $\mathrm{p}$ & $0.020-0.155$ & $\mathrm{Au}$ & $\sigma_{p, 1 p l n}$ & $\sim 30$ & $\begin{array}{l}\text { Radio-Chemical } \\
\text { Techniques }\end{array}$ & $\begin{array}{c}\text { Orsay, } \\
\text { Synchrocyclotron }\end{array}$ & 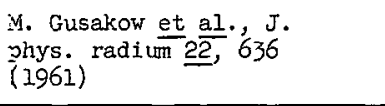 & \\
\hline 373 & $\mathrm{p}$. & $0.040-0.155$ & $A u$ & $\begin{array}{l}\text { Cross sections for } \\
\text { producing } \mathrm{Hg} \\
\text { isotopes }\end{array}$ & $\sim 20$ & $\begin{array}{l}\text { Mass } \\
\text { Separator }\end{array}$ & $\begin{array}{c}\text { Orsay, } \\
\text { Symchrocyclotron }\end{array}$ & $\begin{array}{l}\text { G. Albouy, M. Gusakow, } \\
\text { and N. Poffe, Rutherford } \\
\text { Jubilee Conf. Manchester } \\
\text { 1961, Paper C } 1 / 26191 .\end{array}$ & \\
\hline $37 L$ & $\mathrm{p}$ & 0.040 to 0.15 & $\mathrm{Au}$ & $\begin{array}{l}\sigma_{p}, 1 p l n \\
\sigma_{p, l p 2 n} \\
\sigma_{p, 3 n} \\
\end{array}$ & 5 & $\begin{array}{l}\text { Radio-Chemical } \\
\text { Methods }\end{array}$ & $\begin{array}{c}\text { Orsay, } \\
\text { Synchrocyclotron }\end{array}$ & $\begin{array}{l}\text { M. Gusakow et al., Compt. } \\
\text { =end. } 251 \text {, } 70 \text { (1960) }\end{array}$ & \\
\hline 379 & $p$ & $0.040-0.155$ & $A u^{197}$ & $\begin{array}{l}\text { Radio-chemical } \\
\text { cross section }\end{array}$ & $\sim 30$ & $\begin{array}{l}\text { Electronag- } \\
\text { netic } \\
\text { Separation }\end{array}$ & $\begin{array}{c}\text { Orsay, } \\
\text { Symchrocyclotron }\end{array}$ & $\begin{array}{l}\text { Iv. Poffé et al., J. phys. } \\
\text { radium 22, } 639(1961)\end{array}$ & \\
\hline 376. & $\mathrm{p}$ & 0.120 & $\mathrm{Au}$ & $\begin{array}{l}\text { Cross section for } \\
\text { production of } \mathrm{Na}^{24} \\
\text { and } \mathrm{P}^{32}\end{array}$ & - & $\begin{array}{l}\text { Radio-Chemical } \\
\text { Methods }\end{array}$ & $\begin{array}{l}\text { Institute for } \\
\text { Nuclear Problems, } \\
\text { Synchrocyclotron }\end{array}$ & $\begin{array}{l}\text { A.K. Lavrukhina et } \\
\text { Atomic Energy } 3, \frac{\text { al., }}{1087} \\
\text { (1957) }\end{array}$ & \\
\hline
\end{tabular}




\begin{tabular}{|c|c|c|c|c|c|c|c|c|c|}
\hline No. & $\begin{array}{l}\text { Incident } \\
\text { Particle }\end{array}$ & $\begin{array}{c}\text { Incident } \\
\text { Particle } \\
\text { Energy (Gev) }\end{array}$ & Target & Measured Quantity & $\begin{array}{l}\text { Estimate of } \\
\text { Experimental } \\
\text { Error }(\phi)\end{array}$ & Detector & $\begin{array}{l}\text { Installation and } \\
\text { Accelerator }\end{array}$ & References & Comments \\
\hline 377 & $\underline{p}$ & 0.154 & $A u^{197}$ & $\begin{array}{l}\text { Energy spectra of } \\
\text { protons, deuterons, .. } \\
\text { and tritons emitted } \\
\text { at } 15^{\circ}, 30^{\circ} \text {, and } 60^{\circ}\end{array}$ & 25 & $\begin{array}{l}\mathrm{dE} / \mathrm{dx}-\mathrm{E} \\
\text { Scintillation } \\
\text { Pelescope }\end{array}$ & Orsay & $\begin{array}{l}\text { J. Génin et ai.', J. } \\
\text { phys. radîm } \\
\text { (1961) }\end{array}$ & .. \\
\hline 378 & $\mathrm{p}$ & 0.155 & $A \cdot x$ & $\begin{array}{l}\sigma_{p},{ }_{n} \\
\sigma_{p, 3 n} \\
\sigma_{p, 4 n}\end{array}$ & 30 & $\begin{array}{l}\text { Radio-Chemical } \\
\text { Techniques }\end{array}$ & $\begin{array}{c}\text { Orsay, } \\
\text { Synchrocyclotron }\end{array}$ & $\begin{array}{l}\text { N. Poffé, M. Riou, and } \\
\text { J. Teillac, Compt. rend. } \\
\text { 248, } 3552 \text { (1959) }\end{array}$ & . \\
\hline 379 & $\mathrm{p}$ & 0.155 & $\mathrm{Au}$ & $\begin{array}{l}\text { Radio-chemical } \\
\text { cross sections } \\
\text { for } \mathrm{Hg} \text { isotope }\end{array}$ & 30 & $\begin{array}{l}\text { Electro- } \\
\text { magnetic } \\
\text { Separation }\end{array}$ & $\begin{array}{c}\text { Orsay, } \\
\text { Synchrocyclotron }\end{array}$ & $\begin{array}{l}\text { N. Poffé et al :' J. } \\
\text { phys, radium } 31 \text {. } 343 \\
(1960)\end{array}$ & \\
\hline 380 & $\mathrm{p}$ & 0.180 & $\mathrm{Au}$ & $\sigma_{\mathrm{pX}}$ & 3 & $\begin{array}{l}\text { Range } \\
\text { Separator }\end{array}$ & $\begin{array}{l}\text { Upsala, } \\
\text { Cyclotron }\end{array}$ & $\begin{array}{l}\text { A. Johansson, U. Svan- } \\
\text { berg, and O. Sundberg, } \\
\text { Arkiv Fysik 19, } 527 \text { (1961) }\end{array}$ & $\begin{array}{c}\text { Poor geometry } \\
\text {. }\end{array}$ \\
\hline 381 & $\mathrm{p}$ & 0.220 & $\mathrm{Au}$ & $\begin{array}{l}\text { Cross section for } \\
\text { production of } \mathrm{Na}^{24} \\
\text { and } P^{32}\end{array}$ & - & $\begin{array}{l}\text { Radio-Chemical } \\
\text { Methods } \\
\end{array}$ & $\begin{array}{l}\text { Institute for } \\
\text { Nuclear Problems, } \\
\text { Synchrocyclotron }\end{array}$ & $\begin{array}{l}\text { A.K. Lavrukhina et al., } \\
\text { Atomic Energy } 3, \frac{91}{1087} \\
\text { (1957) }\end{array}$ & \\
\hline 382 & $\mathrm{p}$ & $0.082-0.426$ & $\mathrm{Au}^{197}$ & $\sigma_{p}$, Ipln & $2-10$ & $\begin{array}{l}\text { Radio-Chemical } \\
\text { Techniques }\end{array}$ & $\begin{array}{c}\text { Chicago, } \\
\text { Synchrocyclotron }\end{array}$ & $\begin{array}{l}\text { H.P. Yule and A. Turkevich, } \\
\text { Phys. Rev. 118, } 1591 \text { (1960) }\end{array}$ & \\
\hline 383 & $\mathrm{p}$ & 0.340 & $\mathrm{Au}$ & $\begin{array}{l}\text { Cross section for } \\
\text { production of } \mathrm{Na}^{24} \\
\text { and } \mathrm{P}^{32}\end{array}$ & .. - & $\begin{array}{l}\text { Radio-Chemical. } \\
\text { Techniques }\end{array}$ & $\begin{array}{l}\text { Institute for } \\
\text { Nuclear Problems, } \\
\text { Synchrocyclotron }\end{array}$ & $\begin{array}{l}\text { A.K. Lavrukhina et.al., } \\
\text { Atomic Energy } 2,1087 \\
\text { (1957) }\end{array}$ & $\ldots$ \\
\hline 384 & $p$ & 0.450 & $\begin{aligned} & \text { Su } \\
. . & .\end{aligned}$ & $\begin{array}{c}\sigma_{p, x \pi^{+} y}\left(E_{\pi^{+}}, \psi_{\pi^{+}}\right) \\
\sigma_{p, x \pi^{-}-y}\left(E_{\pi^{-}} ; \psi_{\pi^{-}}\right) \\
\text {with } \psi_{\pi^{ \pm}}=21.5^{\circ}, 60^{\circ}\end{array}$ & $2-40$ & $\begin{array}{l}\text { Counter- } \\
\text { Teles:ope }\end{array}$ & $\begin{array}{l}\text { Chicago, } \\
\text { Synchrocyclotron }\end{array}$ & $\begin{array}{l}\text { E. Lillethun, Phys. Rev. } \\
125,665(1962)\end{array}$ & Good geometry \\
\hline
\end{tabular}




\begin{tabular}{|c|c|c|c|c|c|c|c|c|c|}
\hline No. & $\begin{array}{l}\text { Incident } \\
\text { Farticle }\end{array}$ & $\begin{array}{c}\text { Incident } \\
\text { Particle } \\
\text { Energy (Gev) }\end{array}$ & Target & Measured Quantity & $\begin{array}{l}\text { Estimate of } \\
\text { Experimental } \\
\text { Error }(\phi)\end{array}$ & Detector & $\begin{array}{l}\text { Installation and } \\
\text { Accelerator }\end{array}$ & References & Comments \\
\hline 385 & $\mathrm{p}$ & c. 480 & $\mathrm{Au}$ & $\begin{array}{l}\text { Cross section for } \\
\text { production of } \mathrm{Na}^{24} \\
\text { and } \mathrm{P}^{32}\end{array}$ & - & $\begin{array}{l}\text { Radio-Chemical } \\
\text { Techniques }\end{array}$ & $\begin{array}{l}\text { Institute for } \\
\text { Nuclear Problems, } \\
\text { Synchrocyclotron }\end{array}$ & $\begin{array}{l}\text { A.K. Lavrukhina et al., } \\
\text { Atomic Energy } 3, \frac{10}{1087} \\
\text { i.1957) }\end{array}$ & \\
\hline 386 & $\mathrm{p}$ & 0.660 & $\mathrm{Au}$ & $\begin{array}{l}\text { Cross section for } \\
\text { production of } \mathrm{Na}^{24} \\
\text { and } \mathrm{P}^{3 ?}\end{array}$ & - & $\begin{array}{l}\text { Radio-Chemical } \\
\text { Techniques }\end{array}$ & $\begin{array}{l}\text { Institute for } \\
\text { Nuclear Problems, } \\
\text { Synchrocyclotron }\end{array}$ & $\begin{array}{l}\text { A.K. Lavrukhina et } \\
\text { fomic Energy } 3, \frac{a l .,}{1087} \\
\text { (1957) }\end{array}$ & \\
\hline 387 & $\mathrm{p}$ & 1.0 & An & Production of $\mathrm{Be}^{7}$ & $\sim 25$ & $\begin{array}{l}\text { Radio-Chemical } \\
\text { Techniques }\end{array}$ & $\begin{array}{l}\text { Brookhaven, } \\
\text { Cosmotron }\end{array}$ & $\begin{array}{l}\text { Ð. Baker, G. Friedlander, } \\
\text { and J. Hucis, Phys. Rev. } \\
\text { 112 } 1319 \text { (1958). }\end{array}$ & \\
\hline 388 & $\mathrm{p}$ & 2.2 & A:I & Production of $\mathrm{Be}^{7}$ & $\sim 25$ & $\begin{array}{l}\text { Radio-Chemical } \\
\text { Techniques }\end{array}$ & $\begin{array}{l}\text { Brookhaven, } \\
\text { Cosmotron }\end{array}$ & $\begin{array}{l}\text { E. Baker, G. Friedlander, } \\
\text { and J. Hudis, Phys. Rev. } \\
112,1319 \text { (1958) }\end{array}$ & \\
\hline 389 & $\mathrm{p}$ & 3.0 & $\mathrm{Au}$ & Production of $\mathrm{Be}^{7}$ & $\sim 25$ & $\begin{array}{l}\text { Radio-Chemical } \\
\text { Techniques }\end{array}$ & $\begin{array}{l}\text { Brookhaven, } \\
\text { Cosmotron }\end{array}$ & $\begin{array}{l}\text { E. Baker, G. Friedlander, } \\
\text { and J. Hudis, Phys. Rev. } \\
112 \text { i319 (1958) }\end{array}$ & \\
\hline 390 & $p$ & 0.061 & $\begin{array}{c}\mathrm{Pb} \\
\text { (thin) }\end{array}$ & $\begin{array}{l}{ }^{\circ} \mathrm{pT} \\
\sigma_{\mathrm{pX}}\end{array}$ & 6 & $\begin{array}{l}\text { Plastic } \\
\text { Scintillators }\end{array}$ & Minnesota. & $\begin{array}{l}\text { v. Meyer, R.M. Eisberg, } \\
\text { and R.F. Carlson, Phys. } \\
\text { Rev. 117, } 1334 \text { (1960) }\end{array}$ & \\
\hline 391 & $\mathrm{p}$ & 0.077 & $\mathrm{Fb}$ & $\sigma_{\mathrm{FX}}$ & $\sim 3$ & $\begin{array}{l}\text { Range } \\
\text { Telescope }\end{array}$ & $\begin{array}{l}\text { Harvard, } \\
\text { Cyclotron }\end{array}$ & $\begin{array}{l}\text { R. Goloskie and } \mathrm{K} \text {. } \\
\text { Strauch, Nuclear Phys. } \\
\text { 29, } 474 \text { (1962) }\end{array}$ & Good geometry \\
\hline 392 & $\mathrm{p}$ & 0.095 & Fo & ${ }^{\sigma} \mathrm{pX}$ & $\sim 3$ & $\begin{array}{l}\text { Range } \\
\text { Telescope }\end{array}$ & $\begin{array}{l}\text { Harvard, } \\
\text { Cyclotron }\end{array}$ & $\begin{array}{l}\text { R. Goloskie and K. } \\
\text { Strauch, Nuclear Phys. } \\
\text { 29, } 474 \text { (1962) }\end{array}$ & Good geometry \\
\hline
\end{tabular}




\begin{tabular}{|c|c|c|c|c|c|c|c|c|c|}
\hline No. & $\begin{array}{l}\text { Incident } \\
\text { Particle }\end{array}$ & $\begin{array}{l}\text { Incident } \\
\text { Particle } \\
\text { Energy (Gev) }\end{array}$ & Tarizet & Measured Quantity & $\begin{array}{l}\text { Estimate of } \\
\text { Experimental } \\
\text { Error }(\phi)\end{array}$ & Detector & $\begin{array}{l}\text { Installation and } \\
\text { Accelerator }\end{array}$ & References & Comments \\
\hline 393 & $p$ & 0.096 & $\mathrm{~Pb}$ & $\sigma_{p, x p y}\left(E_{p}, 40^{\circ}\right)$ & 5 & $\begin{array}{l}\text { Range } \\
\text { Telescope }\end{array}$ & $\begin{array}{l}\text { Harvard, } \\
\text { Cyclotron }\end{array}$ & $\begin{array}{l}\text { K. Strauch and F. Titus, } \\
\text { Phys. Rev. 104; } 191 \text { (1956) }\end{array}$ & . \\
\hline 394 & $\mathrm{p}$ & 0.113 & $\mathrm{~Pb}$ & $\sigma_{p X}$ & $\sim 3$ & $\begin{array}{l}\text { Range } \\
\text { Telescope }\end{array}$ & $\begin{array}{l}\text { Harvard, } \\
\text { Cyclotron }\end{array}$ & $\begin{array}{l}\text { R. Goloskie and K. } \\
\text { Strauch, Nuclear Phys, } \\
\text { 29, } 474 \text { (1962). }\end{array}$ & Good geometry \\
\hline 395 & $\mathrm{p}$ & 0.133 & $\mathrm{~Pb}$ & $\cdot{ }_{\mathrm{pX}}^{\sigma}$ & $\sim 3$ & $\begin{array}{l}\text { Range } \\
\text { Tëlescope }\end{array}$ & $\begin{array}{l}\text { Harvard, } \\
\text { Cyclötron }\end{array}$ & $\begin{array}{l}\text { R. Goloskie and K. } \\
\text { Strauch; Nuclear Phys. } \\
\text { 29, } 474 \text { (1962) }\end{array}$ & Good geometry \\
\hline 396. & $\mathrm{p}$ & 0.143 & $\mathrm{~Pb}$ & $\begin{array}{l}\text { Neutron spectra at } \\
\text { zero degrees }\end{array}$ & $3-10$ & $\begin{array}{l}\text { Neutron Time- } \\
\text { of-Flight } \\
\text { Spectrometer }\end{array}$ & $\begin{array}{c}\text { Earwell, } \\
\text { Synchrocyclotron }\end{array}$ & $\begin{array}{l}\text { P.H. Bowen et al., Nuclear } \\
\text { Phys. 30, } 4 \frac{7}{(1962)}\end{array}$ & \\
\hline 397 & $p$ & 0.160 & $\mathrm{~Pb}$ & $\begin{array}{c}\sigma_{p, x p y}\left(E_{p}, \psi_{p}\right) \\
\text { for } E_{p}<23 \mathrm{Mev} \\
\text { with } \psi_{p}=60^{\circ}, 90^{\circ}, 120^{\circ}\end{array}$ & 10-20 & $\begin{array}{l}\text { Range } \\
\text { Telesc-ope }\end{array}$ & $\begin{array}{l}\text { Harvard, } \\
\text { Cyclotron }\end{array}$ & $\begin{array}{l}\text { R. Fox and N.F. Ramsey, } \\
\text { Phys. Rev. 125, } 1609 \\
(1962)\end{array}$ & \\
\hline 398. & $\mathrm{p}$ & 0.450 & $\mathrm{~Pb}$ & $\begin{array}{c}\sigma_{p, x \pi^{+} y}\left(E_{\pi^{+}}, \psi_{\pi^{+}}\right) \\
\sigma_{p, x \pi^{-} y}\left(E_{\pi^{-}}, \psi_{\pi^{-}}\right) \\
\text {with } \psi_{\pi^{+}}=21.5^{\circ}, 60^{\circ}\end{array}$ & $2-40$ & $\begin{array}{l}\text { Counter. } \\
\text { Telescope }\end{array}$ & $\begin{array}{c}\text { Chicago, } \\
\text { Synchrocyclotron }\end{array}$ & $\begin{array}{c}\text { E. Lillethun, Phys. } \\
\text { Rev. 125, } 665 \text { (1962) } \\
. .\end{array}$ & Good geometry \\
\hline 399 & p & $0.185-0.870$ & Pt & $\sigma_{\mathrm{pX}}$ & io & - & - & $\begin{array}{l}\text { G.P. Millburn et al. } \\
\text { Phys. Rev. } 25 \text {, } \\
\text { (1954). }\end{array}$ & A compilation \\
\hline 400 & $\mathrm{p}$ & $0.300-0.820$ & $\mathrm{~Pb}$ & Neutrons produced & $10-20$ & $\begin{array}{l}\text { Paraffin Block, } \\
\mathrm{B}^{2} \mathrm{O}_{\mathrm{F}_{3}} \text { Counters }\end{array}$ & $\begin{array}{l}\text { Chalk River; Ex- } \\
\text { posure Made at } \\
\text { Echo Lake; Cal. }\end{array}$ & $\begin{array}{l}\text { M. Bercovitch et al., } \\
\text { Phys. Rev. } 119 \text {, } 4 \frac{12}{12} \\
\text { (1960) }\end{array}$ & $\therefore \ldots$ \\
\hline
\end{tabular}




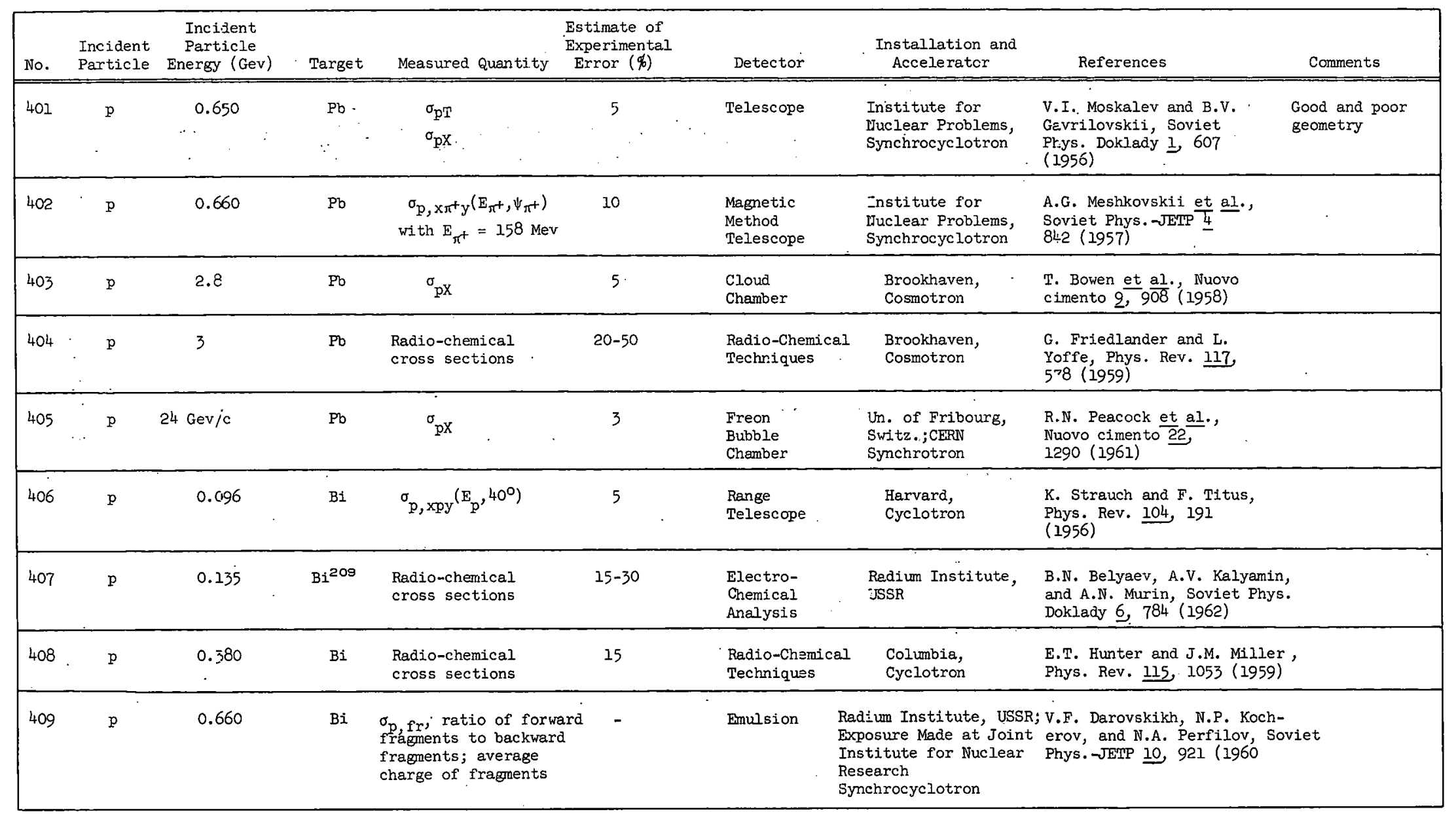




\begin{tabular}{|c|c|c|c|c|c|c|c|c|c|}
\hline No. & $\begin{array}{l}\text { Incident } \\
\text { Particle }\end{array}$ & $\begin{array}{c}\text { Incident } \\
\text { Particle } \\
\text { Energy (Gev) }\end{array}$ & Target & Measured Quantity. & $\begin{array}{l}\text { Estimate of } \\
\text { Experimental } \\
\text { Error }(\%)\end{array}$ & Detector & $\begin{array}{l}\text { Installation and } \\
\text { Accelerator }\end{array}$ & - References & Comments \\
\hline 410 & p & 0.660 & $\mathrm{Bi}$ & $\begin{array}{l}\text { Radio-chemical } \\
\text { cross: sections } \\
\quad . \quad \ldots\end{array}$ & - & $\begin{array}{l}\text { Radio-Chemical } \\
\text { Techniques } \\
\end{array}$ & $\begin{array}{l}\text { Joint Instit ute } \\
\text { for Nuclear } \\
\text { Research, } \\
\text { Synchrocyclotron }\end{array}$ & $\begin{array}{l}\text { T.V. Malysheva and I.P. } \\
\text { Alimarin, Soviet Phys.- } \\
\text { JETP 8, } 772 \text { (1959) }\end{array}$ & \\
\hline 411 & $p$ & 0.120 & Th & $\begin{array}{l}\text { Cross section for } \\
\text { production of } \mathrm{Na}^{24} \\
\text { and } \mathrm{P}^{32}\end{array}$ & - & $\begin{array}{l}\text { Radio-Chemical } \\
\text { Techniques }\end{array}$ & $\begin{array}{l}\text { Institute for } \\
\text { Nuclear Problems, } \\
\text { Synchrocyclotron }\end{array}$ & $\begin{array}{l}\text { A.K. Lavrukhina et al., } \\
\text { Atomic Energy } 2,1087 \text {. } \\
\text { (1957) }\end{array}$ & . \\
\hline 412 & $\mathrm{p}$ & 0.150 & Th & $\begin{array}{l}\text { Tritium production } \\
\text { cross section }\end{array}$ & $\sim 10$ & $\begin{array}{l}\text { Radio-Chemical } \\
\text { Techniques }\end{array}$ & $\begin{array}{l}\text { Orsay, } \\
\text { Synchrocyclotron }\end{array}$ & $\begin{array}{l}\text { C. Brun, M: Lefort, and } \\
\text { X. Tarrago, J. phys. } \\
\text { radium 23, } 167 \text { (1962) }\end{array}$ & . \\
\hline 413 & $p$ & 0.150 & $\mathrm{Th}^{232}$ & $\begin{array}{l}\text { Production of } \\
\text { alpha particles }\end{array}$ & $5-30$ & $\begin{array}{l}\text { Radio-Chemical } \\
\text { Techniques }\end{array}$ & $\begin{array}{c}\text { Orsay, } \\
\text { Symchrocyclotron }\end{array}$ & $\begin{array}{l}\text { M. Lefort, G. Simionoff, and } \\
\text { X. Tarrago, J. phys. radium } \\
\text { 21, } 338 \text { (1960) }\end{array}$ & \\
\hline 414 & $p$ & 0.155 & $\mathrm{Th}^{232}$ & $a_{p, 6 n}$ & 15 & $\begin{array}{l}\text { Radio-Chemical } \\
\text { Techniques - }\end{array}$ & - & $\begin{array}{l}\text { G. Bouissieres et } \\
\text { Compt.. rend. } \frac{251}{11} \cdot \frac{1}{21}{ }^{5} \\
\text { (1960) }\end{array}$ & . \\
\hline $415^{\circ}$ & $p$ & $0.155^{\circ}$ & Th & $\dot{\sigma}_{p, \alpha \times n}$ & $10-30$ & $\begin{array}{l}\text { Radio-Chemical } \\
\text { Techniques }\end{array}$ & $\begin{array}{l}\text { Orsay, } \\
\text { Synchrocyclotron }\end{array}$ & $\begin{array}{l}\text { X. 'Tarrago, J. phys, radium } \\
23 \text {, I-6 (1962) }\end{array}$ & \\
\hline 436 & p. . & 0.220 & Th & $\begin{array}{l}\text { Cross section for } \\
\text { production of } \mathrm{Na}^{24} \\
\text { and } \mathrm{P}^{32}\end{array}$ & - & $\begin{array}{l}\text { Radio-Chemical } \\
\text { Methods }\end{array}$ & $\begin{array}{l}\text { Institute for } \\
\text { Nuclear Probloms, } \\
\text { Synchrocyclotron }\end{array}$ & $\begin{array}{l}\text { A.K. Lavrukhina et al. } \\
\text { Atomic "Energy } 2,1087 \\
\text { (1957) }\end{array}$ & $\cdots$ \\
\hline 417 & $p$ & 0.340 & $\mathrm{Th}$ & Neutron yield & 10 & $\begin{array}{l}\mathrm{MnSO}_{4} \\
\text { Solution. }\end{array}$ & $\begin{array}{l}\text { Berkeley and } \\
\text { Chicago Cyclotrons }\end{array}$ & $\begin{array}{l}\text { W.E. Crandall and G.P. } \\
\text { Miliburn, J. Appl. Phys. } \\
\text { 29 } 698 \text { (1958) }\end{array}$ & : \\
\hline
\end{tabular}




\begin{tabular}{|c|c|c|c|c|c|c|c|c|c|}
\hline No. & $\begin{array}{l}\text { Incident } \\
\text { Particle }\end{array}$ & $\begin{array}{c}\text { Incident } \\
\text { Particle } \\
\text { Energy (Gev) }\end{array}$ & Target & Measured Quantity & $\begin{array}{l}\text { Estimate of } \\
\text { Experimental } \\
\text { Error }(\%)\end{array}$ & Detector & $\begin{array}{l}\text { Installation and } \\
\text { Accelerator }\end{array}$ & References & Comments \\
\hline 418 & $\mathrm{p}$ & 0.340 & Th & $\begin{array}{l}\text { Cross section for } \\
\text { production of } \mathrm{Na}^{24} \\
\text { and } \mathrm{P}^{32}\end{array}$ & - & $\begin{array}{l}\text { Radio-Chemical } \\
\text { Methods }\end{array}$ & $\begin{array}{l}\text { Institute for } \\
\text { 1juclear Problems, } \\
\text { Synchrocyclotron }\end{array}$ & $\begin{array}{l}\text { A.K. Lavrukiina et al., } \\
\text { Atomic-Energy 3, } 1087 \\
\text { (1957) }\end{array}$ & \\
\hline 419 & $\mathrm{p}$ & 0.480 & Th & $\begin{array}{l}\text { Cross section for } \\
\text { production of } \mathrm{Na}^{24} \\
\text { and } \mathrm{P}^{32}\end{array}$ & - & $\begin{array}{l}\text { Radio-Chemical } \\
\text { Methods }\end{array}$ & $\begin{array}{l}\text { Institute for } \\
\text { Wuclear Problems, } \\
\text { Synchrocyclotron }\end{array}$ & $\begin{array}{l}\text { A.K. Lavrukinina et al., } \\
\text { Atomic Energy 3, 1087 } \\
(=957)\end{array}$ & \\
\hline 420 & $\mathrm{p}$ & 0.660 & Th & $\begin{array}{l}\text { Cross section for } \\
\text { production of } \mathrm{Na}^{24} \\
\text { and } \mathrm{P}^{32}\end{array}$ & - & $\begin{array}{l}\text { Radio-Chemical } \\
\text { Methods }\end{array}$ & $\begin{array}{l}\text { Institute for } \\
\text { Nuclear Problems, } \\
\text { Synchrocyclotron }\end{array}$ & $\begin{array}{l}\text { A.K. Lavrukhina et al. } \\
\text { Atomic Energy } 3, \frac{1087}{(1957)}\end{array}$ & \\
\hline 421 & $\mathrm{p}$ & 0.68 & Th & $\begin{array}{l}\text { Radio-chemical } \\
\text { cross sections }\end{array}$ & 20 & $\begin{array}{l}\text { Radio-Chemical } \\
\text { Techniques }\end{array}$ & $\begin{array}{l}\text { 3rookhaven, } \\
\text { Cosmotron; Berkeley, } \\
\text { 3evatron and } \\
\text { Synchrocyclotron }\end{array}$ & 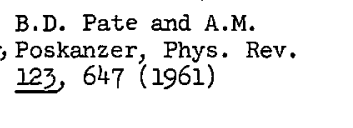 & \\
\hline 422 & $\mathrm{p}$ & 1.80 & Th & $\begin{array}{l}\text { Radio-chemical } \\
\text { cross sections }\end{array}$ & 20 & $\begin{array}{l}\text { Radio-Chemical } \\
\text { Techniques }\end{array}$ & $\begin{array}{l}\text { Brookhaven, } \\
\text { Zosmotron; Berkeley, } \\
\text { Bevatron and } \\
\text { Synchrocyclotron }\end{array}$ & $\begin{array}{l}\text { B.D. Pate. and A.M. } \\
\text { Poskanzer, Phys. Rev. } \\
\text { 123, } 647 \text { (1961) }\end{array}$ & \\
\hline 423 & $\mathrm{p}$ & 0.030 to 0.150 & $U^{238}$ & $\sigma_{p, 3 n}$ & $15-25$ & $\begin{array}{l}\text { Radio-Chemical } \\
\text { Methods }\end{array}$ & $\begin{array}{c}\text { Orsay, } \\
\text { Synchrocyclotron }\end{array}$ & $\begin{array}{l}\text { M. Lefort, Compt. rend. } \\
253 \text {, 2221 (1961) }\end{array}$ & \\
\hline 424 & $\mathrm{p}$ & 0.143 & U & $\begin{array}{l}\text { Neutron spectrum at } \\
\text { zero degrees }\end{array}$ & $3-10$ & $\begin{array}{l}\text { Neutron Time- } \\
\text { of-Flight } \\
\text { Spectrometer }\end{array}$ & $\begin{array}{c}\text { Harwell, } \\
\text { Synchrocyclotron }\end{array}$ & $\begin{array}{l}\text { P.H. Bowen et al., } \\
\text { Nuclear Phy-s. } 30 \text {, } \\
4.75 \text { (1962) }\end{array}$ & \\
\hline 425 & $\mathrm{p}$ & 0.340 & U & Neutron yield & 10 & $\begin{array}{l}\mathrm{MnSO}_{4} \\
\text { Solution }\end{array}$ & $\begin{array}{l}\text { Berkeley and } \\
\text { Chicago Cyclotrons }\end{array}$ & $\begin{array}{l}\text { W.E. Crandall and G.P. } \\
\text { Miliburn, J. Appl. Phys. } \\
\text { 29, } 698 \text { (1958) }\end{array}$ & \\
\hline
\end{tabular}

$$
\because \cdots, \ldots
$$




\begin{tabular}{|c|c|c|c|c|c|c|c|c|c|}
\hline No. & $\begin{array}{l}\text { Incident } \\
\text { Particle }\end{array}$ & $\begin{array}{c}\text { Incident } \\
\text { Particle } \\
\text { Energy (Gev) }\end{array}$ & Target & Measured Quantity & $\begin{array}{l}\text { Estimate of } \\
\text { Experimental } \\
\text { Error }(\%)\end{array}$ & Detector & $\begin{array}{l}\text { Installation and } \\
\text { Accelerator }\end{array}$ & References & Comments \\
\hline 426 & $\mathrm{p}$ & 0.450 & u & 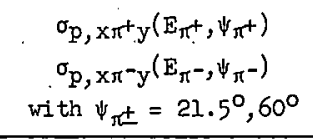 & $2-40$ & $\begin{array}{l}\text { Couniter } \\
\text { Telescope }\end{array}$ & $\begin{array}{l}\text { Chicago, } \\
\text { Synchrocyclotron } \\
\vdots\end{array}$ & $\begin{array}{l}\text { E. Lillethun, Phys. } \\
\text { Rev. 125. } 665 \text { (1962) }\end{array}$ & Good geometry \\
\hline 427 & $\mathrm{p}$ & $0.185-0.870$ & U & $\sigma_{\mathrm{pX}}$ & 10 & - & $\cdot-$ & $\begin{array}{l}\text { G.P. Millburn et al., } \\
\text { Phys. Rev. } 25 \text {, } 1268 \text {. } \\
\text { (1954) }\end{array}$ & A compilation \\
\hline 428 & $p$ & $0.300-0.820$ & $\mathrm{U}$ & Neutrons produced & $10-20$ & $\begin{array}{l}\text { Paraffin Block, } \\
{ }^{1} \mathrm{~F}_{3} \text { Counters }\end{array}$ & $\begin{array}{l}\text { Chalk River; Expo- } \\
\text { sure Made at } \\
\text { Echo Lake, Cal. }\end{array}$ & $\begin{array}{l}\text { M. Bercovitch et al., } \\
\text { Phys. Rev. } 112,412 \\
\text { (1960) }\end{array}$ & \\
\hline 429 & $p$ & 0.650 & $\mathrm{U}$ & $\begin{array}{l}\sigma_{\mathrm{pT}} \\
\sigma_{\mathrm{pX}}\end{array}$ & 5 & Telescope & $\begin{array}{l}\text { Institute for } \\
\text { Nuclear Problems, } \\
\text { Synchrocyclotron }\end{array}$ & $\begin{array}{l}\text { V.I. Moskalev and B.v. } \\
\text { Garilovskii, Soviet Phys. } \\
\text { Doklady 1, } 607 \text { (1956) }\end{array}$ & $\begin{array}{l}\text { Good and poor } \\
\text { geometry }\end{array}$ \\
\hline 430 & $\mathrm{p}$ & 0.660 & $\mathrm{U}$ & $\begin{array}{c}\sigma_{p, x p y}\left(E_{p}, \psi_{p}\right) \\
\text { for } E_{p}>60 \text { Mev with } \\
\psi_{p}=7^{\circ}, 12^{\circ}, 18^{\circ}, 24^{\circ}, 30^{\circ}\end{array}$ & $\begin{array}{l}\text { h } \\
\text { jo }\end{array}$ & $\begin{array}{l}\text { Magnetic } \\
\text { Analyzer }\end{array}$ & $\begin{array}{l}\text { Joint Institute } \\
\text { for Nuclear } \\
\text { Research, Dubna, } \\
\text { Synchrocyclotron }\end{array}$ & $\begin{array}{l}\text { L.s. Azhgirei et al.' } \\
\text { Nuclear Phys. } \frac{\text { al }}{25} \text { (1959) }\end{array}$ & \\
\hline 431 & $p$ & 0.68 & $U^{235}$ & $\begin{array}{l}\text { Radio-chemical } \\
\text { cross sections }\end{array}$ & 20 & $\begin{array}{l}\text { Radio-Chemical } \\
\text { Techniques }\end{array}$ & $\begin{array}{l}\text { Brookhaven, } \\
\text { Cosmotron;Berkeley, } \\
\text { Bevatron and } \\
\text { Synchrocyclotron }\end{array}$ & $\begin{array}{l}\text { B.D. Pate and A.M. } \\
\text { Poskanzer, Phys. Rev. } \\
\text { 123. } 647 \text { (1961) }\end{array}$ & · \\
\hline 432 & $\mathrm{p}$ & 0.68 & $u^{238}$ & $\begin{array}{l}\text { Radio-chemical } \\
\text { cross sections }\end{array}$ & .20 & $\begin{array}{l}\text { Radio-Chemical } \\
\text { Techniques }\end{array}$ & $\begin{array}{l}\text { Brookhaven, Cosmo- } \\
\text { tron; Berkeley, } \\
\text { Bevatron and } \\
\text { Synchrocyclotron }\end{array}$ & $\begin{array}{l}\text { B.D. Pate and A.M. } \\
\text { Poskanzer, Phys. Rev. } \\
\text { 123, } 647 \text { (1961) }\end{array}$ & \\
\hline
\end{tabular}




\begin{tabular}{|c|c|c|c|c|c|c|c|c|c|}
\hline No. & $\begin{array}{l}\text { Incident } \\
\text { Particle }\end{array}$ & $\begin{array}{c}\text { Incident } \\
\text { Particle } \\
\text { EnerEy (Gev) }\end{array}$ & Target & Measured Quantity & $\begin{array}{l}\text { Estimate of } \\
\text { Experimental } \\
\text { Error }(\phi)\end{array}$ & Detector & $\begin{array}{l}\text { Installation and } \\
\text { Accelerator }\end{array}$ & References & Comments \\
\hline 433 & $\mathrm{p}$ & 1.00 & $u^{238}$ & $\begin{array}{l}\text { Radio-chemical } \\
\text { cross sections }\end{array}$ & 20 & $\begin{array}{l}\text { Radio-Chemical } \\
\text { Techniques }\end{array}$ & $\begin{array}{l}\text { Brookhaven, Cosmo- } \\
\text { tron; Berkeley, } \\
\text { Bevatron and } \\
\text { Synchrocyclotron }\end{array}$ & $\begin{array}{l}\text { B.D. Pate end A.M. } \\
\text { Poskanzer, Phys. Rev. } \\
\text { 123, } 647(1961)\end{array}$ & \\
\hline 434. & $\mathrm{p}$ & 1.80 & $u^{235}$ & $\begin{array}{l}\text { Radio-chemical } \\
\text { cross sections }\end{array}$ & 20 & $\begin{array}{l}\text { Radio-Chemical } \\
\text { Techniques }\end{array}$ & $\begin{array}{l}\text { Brookhaven, Cosmo- } \\
\text { tron; Berkeley, } \\
\text { Bevatron and } \\
\text { Synchrocyclotron }\end{array}$ & $\begin{array}{l}\text { B.D. Pate and A.M. } \\
\text { P.oskanzer, Phys. Rev. } \\
\text { 123, } 647(1961)\end{array}$ & \\
\hline 435 & p & 1.80 & $\mathrm{U}^{235}$ & $\begin{array}{l}\text { Radio-chemical } \\
\text { cross səctions }\end{array}$ & 20 & $\begin{array}{l}\text { Radio-Chemical } \\
\text { Techniques }\end{array}$ & $\begin{array}{l}\text { Brookhaven, Cosmo- } \\
\text { tron; Berkeley, } \\
\text { Bevatron and } \\
\text { Synchrocyclotron }\end{array}$ & $\begin{array}{l}\text { B.D. Pate and A.M. } \\
\text { Poskanzer, Phys. Rev. } \\
\text { 123, } 647 \text { (1961) }\end{array}$ & \\
\hline 436 & $\mathrm{p}$ & 3 & $\mathrm{U}$ & $\begin{array}{l}\text { Radio-cinemical } \\
\text { cross sections }\end{array}$ & $20-50$ & $\begin{array}{l}\text { Radio-Chemical } \\
\text { Techniques }\end{array}$ & $\begin{array}{l}\text { Brookhaven, } \\
\text { Cosmotron }\end{array}$ & $\begin{array}{l}\text { G. Friedlander and L. } \\
\text { Yoffe, Phys. Rev. } 117 \\
578 \text { (1959) }\end{array}$ & \\
\hline 437 & $\mathrm{p}$ & 6.20 & $u^{238}$ & $\begin{array}{l}\text { Radio-chemical } \\
\text { cross sections }\end{array}$ & 20 & $\begin{array}{l}\text { Radio-Chemical } \\
\text { Techniques }\end{array}$ & $\begin{array}{l}\text { Brookhaven, Cosmo- } \\
\text { tron; Berkeley, } \\
\text { Bevatron and } \\
\text { S-mchrocyclotron }\end{array}$ & $\begin{array}{l}\text { B.D. Pate and A.M. } \\
\text { Poskanzer, Phys. Rev. } \\
\text { 123, } 647(1961)\end{array}$ & \\
\hline 438 & $\mathrm{p}$ & 0.100 & $\begin{array}{l}\text { Light Brul- } \\
\text { sion Nuclei }\end{array}$ & $\begin{array}{l}\quad \sigma_{\mathrm{p}, \mathrm{f}}(\mathrm{z}>2) \\
\text { Energy and angular } \\
\text { distribution of frac } \\
\text { ments in light and } \\
\text { heavy emulsion nucle }\end{array}$ & $\begin{array}{l}40 \\
\text { Lei }\end{array}$ & $\begin{array}{l}\text { Rad } \\
\text { pos } \\
\text { Ins } \\
\text { lea } \\
\text { Syn }\end{array}$ & $\begin{array}{l}\text { di:m Institute; Ex- } \\
\text { sure Made at Joint } \\
\text { stitute for Nuc- } \\
\text { ar Research } \\
\text { nchrocyclotron }\end{array}$ & $\begin{array}{l}\text { U.R. Arifkhanov et al., } \\
\text { Soviet Phys. - JETP } 1 \text { I } \\
806 \text { (1960) }\end{array}$ & \\
\hline 439 & $p$ & 0.300 & $\begin{array}{l}\text { Light Erul- } \\
\text { sion Nuclei }\end{array}$ & $\sigma_{p, f r}\left(z \geq_{4}\right)$ & $20-50$ & - & $\begin{array}{l}\text { Radium Institute, } \\
\text { USSR }\end{array}$ & $\begin{array}{l}\text { o.v. Lozhkin, Soviet } \\
\text { Phys. JETP б, } 273 \text { (1958) }\end{array}$ & \\
\hline
\end{tabular}




\begin{tabular}{|c|c|c|c|c|c|c|c|c|}
\hline No. & $\begin{array}{l}\text { Incident } \\
\text { Particle }\end{array}$ & $\begin{array}{c}\text { Incident } \\
\text { Particle } \\
\text { Energy (Gev) }\end{array}$ & Target & $\begin{array}{r}E S \\
\text { Ex } \\
\text { Measured Quantity } \quad E\end{array}$ & $\begin{array}{l}\text { Estimate of } \\
\text { Experimental } \\
\text { Error }(\%)\end{array}$ & Detector & $\begin{array}{l}\text { Installation and } \\
\text { Accelerator }\end{array}$ & References \\
\hline 440 & p & 0.350 & $\begin{array}{l}\text { Light Emul- } \\
\text { sion Nuclei }\end{array}$ & $\sigma_{p, f r}(z \geq 4)$ & $20-50$ & - & $\begin{array}{l}\text { Radium Institute, } \\
\text { USSR }\end{array}$ & $\begin{array}{l}\text { O.v. Lozhkin, Soviet } \\
\text { Phys. JETP 6, } 273 \text { (1958) }\end{array}$ \\
\hline 441 & $\mathrm{p}$ & 0.450 & $\begin{array}{l}\text { Light Emul- } \\
\text { sion Nuclei }\end{array}$ & $\left.\sigma_{p}, f r^{(z} \geq 4\right)$ & $20-50$ & - & $\begin{array}{l}\text { Radium Institute, } \\
\text { USSR }\end{array}$ & $\begin{array}{l}\text { o.v. Lozhkin, Soviet Phys. - } \\
\text { JETP } 6,273 \text { (1958 }\end{array}$ \\
\hline 442 & $p$ & $0.560^{\circ}$ & $\begin{array}{l}\text { Light Enul- } \\
\text { sion Nuielei. }\end{array}$ & $\sigma_{p, f r}^{\prime}(z \geq 4)$ & $20-50$ & - & $\begin{array}{l}\text { Radium Institute, } \\
\text { USSR }\end{array}$ & $\begin{array}{l}\text { 0.V. Lozhkin, Soviet Phys. - } \\
\text { JETP 6, } 273 \text { (1958) }\end{array}$ \\
\hline 443 & $p$ & 0.660 & $\begin{array}{l}\text { Light Enul- } \\
\text { sion Nuslei }\end{array}$ & $\sigma_{p, f r}(z \geq 4)$ & $20-50$ & - & $\begin{array}{l}\text { Radium Institute, } \\
\text { USSR }\end{array}$ & $\begin{array}{l}\text { o.V. Lozhkin, Soviet Phys. - } \\
\text { JETP 6, } 273 \text { (1958) }\end{array}$ \\
\hline 444 & $p$ & 0.930 & $\begin{array}{l}\text { Light Enul- } \\
\text { sion Nuslei }\end{array}$ & $\begin{array}{l}\sigma_{p, f r}(2 \geq 4) \text {; angle } \\
\text { and energy distribu- } \\
\text { tion of } L i \text {; angular } \\
\text { distribution of } \\
\text { fragnents }\end{array}$ & $10-40$ & - & $\begin{array}{l}\text { Radium Institute, } \\
\text { USSR; Exposure Made } \\
\text { at Birmingham } \\
\text { Synchrotron }\end{array}$ & $\begin{array}{l}\text { 0.v. Lozhkin et al., } \\
\text { Soviet Phys. -JETP } \\
11,1001 \text { (1960) }\end{array}$ \\
\hline 445 & $p$ & 0.950 & $\begin{array}{l}\text { Light Enul- } \\
\text { sion Nuclei }\end{array}$ & $\begin{array}{l}\text { Number distribu- } \\
\text { tion of protons } \\
\text { emitted in light } \\
\text { and heavy emulsion } \\
\text { nuclei }\end{array}$ & - &.- & $\begin{array}{l}\text { Plates Exposed } \\
\text { at Birmingham }\end{array}$ & $\begin{array}{l}\text { G. Philbert, Compt. rend. } \\
243 \text {, } 141 \text { (1956) }\end{array}$ \\
\hline 446 & $p$ & 1 & $\begin{array}{l}\text { Light Emul- } \\
\text { sion Nuclei }\end{array}$ & $\begin{array}{l}\text { Charged particle } \\
\text { distribution for } \\
\text { light and heavy } \\
\text { nuclei }\end{array}$ & - & - & $\begin{array}{l}\text { College of France, } \\
\text { Paris; Plates Ex- } \\
\text { posed at Birmingham }\end{array}$ & $\begin{array}{l}\text { G. Philbert, J. phys. } \\
\text { radium 18, } 656 \text { (1957) }\end{array}$ \\
\hline 447 & $p$ & 1 & $\begin{array}{l}\text { Light Emul- } \\
\text { sion Niclei }\end{array}$ & $\begin{array}{l}\text { Prong distribu- } \\
\text { tion }\end{array}$ & - & - & $\begin{array}{l}\text { Orsay; Plates Ex- } \\
\text { posed at Birmingham }\end{array}$ & $\begin{array}{l}\text { G. Philberg and L. Vig- } \\
\text { neron, Compt. rend. } 247 \\
290 \text { (1958) }\end{array}$ \\
\hline $448^{\circ}$ & $p$ & 1 & $\begin{array}{l}\text { Light Emul- } \\
\text { sion Nuclei }\end{array}$ & $\begin{array}{l}\text { Angular and e nergy } \\
\text { distribution of } \\
\text { fast protons }\end{array}$ & - & - & $\begin{array}{l}\text { Orsay; Plates } \\
\text { Exposed at } \\
\text { Birmingham }\end{array}$ & $\begin{array}{l}\text { G. Philbert and L. } \\
\text { Vigneron, Compt. rend. } \\
\text { 247, } 1335 \text { (1958) }\end{array}$ \\
\hline
\end{tabular}




\begin{tabular}{|c|c|c|c|c|c|c|c|c|c|}
\hline No. & $\begin{array}{l}\text { Incident } \\
\text { Particle }\end{array}$ & $\begin{array}{l}\text { Incident } \\
\text { Particle } \\
\text { Energs (Gev) }\end{array}$ & Farget & Measured Quantity & $\begin{array}{l}\text { Est:mate of } \\
\text { Experimental } \\
\text { Er:or }(\%)\end{array}$ & Detector & $\begin{array}{l}\text { Installation and } \\
\text { Accelerator }\end{array}$ & References & Comments \\
\hline 449 & $\mathrm{p}$ & 9 & $\begin{array}{l}\text { Ligh= Emul- } \\
\text { sion Nucle: }\end{array}$ & $\mathbb{N}_{s}$ vs. $\mathbb{N}_{h}$ & 10 & - & $\begin{array}{l}\text { Insti-ute of Atom. Phys. } \\
\text { Exposure Made at Bucha- } \\
\text { rest; JIINR } \\
\text { Synchrotron }\end{array}$ & $\begin{array}{l}\text { F. Friedländer, M. Marcu, } \\
\text { and M. Spîrchez, Rev. de } \\
\text { Phys. 6, } 45 T \text { (1961) }\end{array}$ & \\
\hline 450 & $\mathrm{p}$ & $0 .: 00$ & $\begin{array}{l}\text { Heavy Emul- } \\
\text { sion Nucl三i }\end{array}$ & $\begin{array}{l}{ }_{\mathrm{p}}^{\sigma} \operatorname{fr}_{\mathrm{fr}}(\mathrm{z}) \\
\text { Energy and angular } \\
\text { distribution of frag- } \\
\text { ments ir. light and } \\
\text { heavy emulsion nuclei }\end{array}$ & i & - & $\begin{array}{l}\text { Radium Institute; Expo- } \\
\text { sure Made at Joint } \\
\text { Institute for Nuclear } \\
\text { Research } \\
\text { Synchrocylotron }\end{array}$ & $\begin{array}{l}\text { U.R. Arifkhenov et al., } \\
\text { Soviet Phys. -JETP } 1 \text { l] } \\
805 \text { (1960) }\end{array}$ & \\
\hline 451 & $\mathrm{p}$ & 0.130 & $\begin{array}{l}\text { Heavy Emul- } \\
\text { sion Nuclei }\end{array}$ & $\begin{array}{l}\text { Prong distribution } \\
\text { for cascade alphas } \\
\text { and protons. Ex- } \\
\text { citation energy } \\
\text { distribution }\end{array}$ & - & - & $\begin{array}{l}\text { Radium Institute; Expo- } \\
\text { sure Made at Institute } \\
\text { for Nuclear Problens } \\
\text { Synchrocyclotron }\end{array}$ & $\begin{array}{l}\text { V.I. Ostroumov, Soviet } \\
\text { Phys. JETP } 5,12 \text { (1957) }\end{array}$ & \\
\hline 452 & p & .0 .300 & $\begin{array}{l}\text { Heavy Emul- } \\
\text { sion Nuclei }\end{array}$ & $\sigma_{p, f r}(z \geq 4)$ & $20-50$ & - & $\begin{array}{l}\text { Padium Institute, } \\
\text { USSR }\end{array}$ & $\begin{array}{l}\text { o.v. Lozhkin, Soviet Phys.- } \\
\text { JYTP } 6,273(1958)\end{array}$ & \\
\hline 453 & $\mathrm{p}$ & 0.350 & $\begin{array}{l}\text { Heavy Fmil- } \\
\text { sion Nuclei }\end{array}$ & $\left.\sigma_{p, f r}(z) 4\right)$ & $20-50$ & - & $\begin{array}{l}\text { Radium Institute, } \\
\text { USSR }\end{array}$ & $\begin{array}{l}\text { O.v. Lozhkin, Soviet } \\
\text { Phys. JETP 6, } 273 \text { (1958) }\end{array}$ & \\
\hline 454 & $\mathrm{p}$ & $0.350-0.400$ & $\begin{array}{l}\text { Heavy } \\
\text { Emulsior } \\
\text { Nuclei }\end{array}$ & $\begin{array}{l}\text { Black, sparse } \\
\text { black, and gray } \\
\text { prong number dis- } \\
\text { tribution and } \\
\text { angular distribution }\end{array}$ & 15 & - & $\begin{array}{l}\text { Columbia, } \\
\text { Cyclotron }\end{array}$ & $\begin{array}{l}\text { G. Bernardini, E.T. Booth, } \\
\text { ard S.J. Lindenbaum, Phys. } \\
\text { Rev. } 85,826 \text { (1952) }\end{array}$ & \\
\hline 455 & $\mathrm{p}$ & 0.450 & $\begin{array}{l}\text { Heavy Enul- } \\
\text { sior. Nuclei }\end{array}$ & $\sigma_{p, f r}(i z 4)$ & $20-50$ & - & $\begin{array}{l}\text { Radium Institute, } \\
\text { USSR }\end{array}$ & $\begin{array}{l}\text { o.v. Lozhkin, Soviet Phys. - } \\
\text { JEIP } 6,273 \text { (1958) }\end{array}$ & \\
\hline
\end{tabular}




\begin{tabular}{|c|c|c|c|c|c|c|c|c|c|}
\hline No. & $\begin{array}{l}\text { Incident } \\
\text { Particle }\end{array}$ & $\begin{array}{c}\text { Incident } \\
\text { Particle } \\
\text { Energy (Gev) }\end{array}$ & Target & Measured Quantity & $\begin{array}{l}\text { Estimate of } \\
\text { Experimental } \\
\text { Error }(\phi)\end{array}$ & Detector & $\begin{array}{l}\text { Installation and } \\
\text { Accelerator }\end{array}$ & References & Comments \\
\hline 456 & $\mathrm{p}$ & 0.460 & $\begin{array}{l}\text { Heavy Emul- } \\
\text { sion Nuclei }\end{array}$ & $\begin{array}{l}\text { Prong distribution } \\
\text { for cascade alphas } \\
\text { and protons. Ex- } \\
\text { citation energy } \\
\text { distribution }\end{array}$ & - & - & $\begin{array}{l}\text { Radium Institute, } \\
\text { Institute for } \\
\text { Nuclear Problems, } \\
\text { Synchrocyclotron }\end{array}$ & $\begin{array}{l}\text { V.I. Ostroumov, Soviet } \\
\text { Phys. JETP } 5,12 \text { (1957) }\end{array}$ & \\
\hline 457 & $p$ & 0.560 & $\begin{array}{l}\text { Heavo Enul- } \\
\text { sion Nuclei }\end{array}$ & $\sigma_{p, f r}(z \geq 4)$ & $20-50$ & - & $\begin{array}{l}\text { Radium Institute, } \\
\text { USSR }\end{array}$ & $\begin{array}{l}\text { o.v. Lozhkin, Soviet } \\
\text { Phys. JETP } 6 \text {, } 273 \text { (1958) }\end{array}$ & \\
\hline 458 & $\mathrm{p}$ & $0.660^{\circ}$ & $\begin{array}{l}\text { Heavy Emul- } \\
\text { sion Nuclei }\end{array}$ & $\sigma_{p, f r}(z \geq 4)$ & $20-50$ & - & $\begin{array}{l}\text { Radium Institute, } \\
\text { USSR }\end{array}$ & $\begin{array}{l}\text { O.v. Lozhkin, Soviet } \\
\text { Phys. JETP 6, } 273 \text { (1958) }\end{array}$ & \\
\hline 459 & $\mathrm{p}$ & 0.660 & $\begin{array}{l}\text { Heavy En:l- } \\
\text { sion Nuclei }\end{array}$ & $\begin{array}{l}\text { Prong distribution } \\
\text { for cascade alphas } \\
\text { and protons. Ex- } \\
\text { citation energy } \\
\text { distribution }\end{array}$ & - & - & $\begin{array}{l}\text { Radium Institute, } \\
\text { Institute for } \\
\text { Nuclear Problems, } \\
\text { Synchrocyclotron }\end{array}$ & $\begin{array}{l}\text { V.I. Ostroumov, Soviet } \\
\text { Phys. JETP 5, } 12 \text { (1957) }\end{array}$ & \\
\hline 460 & $\mathrm{p}$ & 0.930 & $\begin{array}{l}\text { Heavy Nul- } \\
\text { sion Nuclei }\end{array}$ & $\begin{array}{l}\sigma_{\mathrm{p}, \text { fr }}(\mathrm{z} \geq 4) \text {. Angle } \\
\text { and energy distribu- } \\
\text { tion of } \mathrm{Li} \text {; angular } \\
\text { distribution of } \\
\text { fragnents }\end{array}$ & $10-40$ & - & $\begin{array}{l}\text { Radium Insti tute, } \\
\text { USSR; Exposure Made } \\
\text { at Birmingham } \\
\text { Symchrotron }\end{array}$ & 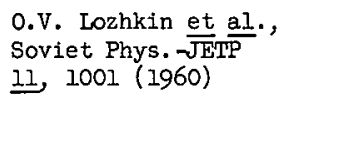 & \\
\hline 461 & $\mathrm{p}$ & 0.950 & $\begin{array}{l}\text { Heavy Enul- } \\
\text { sion Nuciei }\end{array}$ & $\begin{array}{l}\text { Number distribution } \\
\text { of protons emitted } \\
\text { in light and heavy } \\
\text { weight emulsion nuclei }\end{array}$ & - & - & $\begin{array}{l}\text { Plates Exposed } \\
\text { at Birmingham, } \\
\text { Synchrotron }\end{array}$ & $\begin{array}{l}\text { G. Philbert, Compt. rend. } \\
243,141 \text { (1956) }\end{array}$ & \\
\hline 462 & $\mathrm{p}$ & 1 & $\begin{array}{l}\text { Heavy Emul- } \\
\text { sion Nuclei }\end{array}$ & $\begin{array}{l}\text { Energy and angular } \\
\text { distribution of } \\
\text { alpha particles }\end{array}$ & - & - & $\begin{array}{l}\text { Brookhaven } \\
\text { Cosmotron }\end{array}$ & $\begin{array}{l}\text { E.W. Baker, s. Katcoff, } \\
\text { and C.P. Baker, Phys. } \\
\text { Rev. 117 1352 (1960) }\end{array}$ & \\
\hline
\end{tabular}




\begin{tabular}{|c|c|c|c|c|c|c|c|c|c|}
\hline No. & $\begin{array}{l}\text { Ir.cident } \\
\text { Particle }\end{array}$ & $\begin{array}{c}\text { Incident } \\
\text { Particle } \\
\text { Energy (Gev) }\end{array}$ & Target & Measured Quantity & $\begin{array}{l}\text { Estimate of } \\
\text { Experimental } \\
\text { Error }(\phi)\end{array}$ & Detector & $\begin{array}{l}\text { Installation and } \\
\text { Accelerator }\end{array}$ & References & Comments \\
\hline $46 z$ & $p$ & 1 & $\begin{array}{l}\text { Heavy Emil- } \\
\text { sicn Nuclei }\end{array}$ & $\begin{array}{l}\text { Number and angular } \\
\text { distribution of } \\
\text { alphas and light } \\
\text { fragments }\end{array}$ & 25 & - & $\begin{array}{l}\text { Brookhaven, } \\
\text { Cosmotron }\end{array}$ & $\begin{array}{l}\text { E.W. Baker and S Katcoff, } \\
\text { Phys. Rev. 123, } 641(1961)\end{array}$ & . \\
\hline 464 & $p$ & 1 & $\begin{array}{l}\text { Heavy Enul- } \\
\text { sion Nuclei }\end{array}$ & $\begin{array}{l}\text { Charged particle } \\
\text { distribution for } \\
\text { light and heavy } \\
\text { nuclei }\end{array}$ & - & - & $\begin{array}{l}\text { College of France, } \\
\text { Paris; Plates } \\
\text { Exposed at } \\
\text { Birmingham }\end{array}$ & $\begin{array}{l}\text { G. Philbert, J. phys. } \\
\text { radium 18, } 656 \text { (1957) }\end{array}$ & \\
\hline 465 & $\mathrm{p}$ & 2 & $\begin{array}{l}\text { Heary Hmul- } \\
\text { sion Nuclei } \\
\text {. }\end{array}$ & $\begin{array}{l}\text { Energy and angular } \\
\text { distrikution of } \\
\text { alpha Farticles }\end{array}$ & - & - & $\begin{array}{l}\text { Brookhaven, } \\
\text { Cosmotron }\end{array}$ & $\begin{array}{l}\text { E.W. Baker, S. Katcoff, } \\
\text { and C.P. Baker, Phys. Rev. } \\
117,1352(1960)\end{array}$ & \\
\hline 466 & $p$ & 2 & $\begin{array}{l}\text { Heavy Emi:- } \\
\text { sion Nuclei }\end{array}$ & $\begin{array}{l}\text { Number and angular } \\
\text { distribution of } \\
\text { alphas and light } \\
\text { fragments }\end{array}$ & 25 & - & $\begin{array}{l}\text { Brookhaven, } \\
\text { Cosmotron }\end{array}$ & $\begin{array}{l}\text { E.W. Baker and S. Katcoff, } \\
\text { Pnys. Rev. 123, } 641 \text { (1961) }\end{array}$ & \\
\hline 467 & $\mathrm{p}$ & 3 & $\begin{array}{l}\text { Heary mul- } \\
\text { sior. Nuciei }\end{array}$ & $\begin{array}{l}\text { Energy and angular } \\
\text { distribution of } \\
\text { alpha particles }\end{array}$ & - & - & $\begin{array}{l}\text { Brookhaven, } \\
\text { Cosmotron }\end{array}$ & $\begin{array}{l}\text { E.W. Baker, S. Katcoff, } \\
\text { and C.P. Baker, Phys. Rev. } \\
\text { 117, } 1352(1960)\end{array}$ & \\
\hline 468 & $\mathrm{p}$ & 3 & $\begin{array}{l}\text { Heavy Emul- } \\
\text { sion Nuclei }\end{array}$ & $\begin{array}{l}\text { Number and angular } \\
\text { distribution of } \\
\text { alphas and light } \\
\text { fragments }\end{array}$ & 25 & - & $\begin{array}{l}\text { Brookhaven, } \\
\text { Cosmotron }\end{array}$ & $\begin{array}{l}\text { E. W. Baker and S. Katcoff, } \\
\text { Phys. Rev. } 123,641 \text { (1961) }\end{array}$ & \\
\hline 469 & $\mathrm{p}$ & 9.0 & $\begin{array}{l}\text { Heavy Emil- } \\
\text { sion Nuclei }\end{array}$ & $\begin{array}{l}\text { Energy spectra of } \\
\text { fast charged } \pi^{+} \text {. } \\
\text { A ngular distribution } \\
\text { of fast charged } \pi^{1} s \\
\text { and protons. Energy } \\
\text { vs. angle of fast } \pi^{\prime} s\end{array}$ & $\begin{array}{l}10-20 \\
y^{2}\end{array}$ & - & $\begin{array}{l}\text { Eoint Institute } \\
\text { for Nuclear } \\
\text { Research, } \\
\text { Synchrotron }\end{array}$ & $\begin{array}{l}\text { N.P. Bogachev et al., } \\
\text { Sc.viet Phys. -JWTP } \\
317 \text { (1960) }\end{array}$ & \\
\hline
\end{tabular}




\begin{tabular}{|c|c|c|c|c|c|c|c|c|c|}
\hline No. & $\begin{array}{l}\text { Incident } \\
\text { Particle }\end{array}$ & $\begin{array}{c}\text { Incident } \\
\text { Particle } \\
\text { Energy (Gev) }\end{array}$ & Target & Measured Quantity $\quad \begin{array}{c}\text { Est } \\
\text { Er }\end{array}$ & $\begin{array}{l}\text { timate of } \\
\text { cperimental } \\
\text { irror }(\phi)\end{array}$ & Detector & $\begin{array}{l}\text { Installation and } \\
\text { Accelerator }\end{array}$ & References & Corments \\
\hline 470 & $p$ & 9 & $\begin{array}{l}\text { Heavy Emul- } \\
\text { sion Nuclei }\end{array}$ & $\begin{array}{l}\text { Frequency of emis- } \\
\text { sion of } \mathrm{Li}^{8} \text {, } \mathrm{Li}^{9} \text {, and } \\
\mathrm{B}^{\mathrm{B}} \text { fragnents for } \\
\mathrm{N}_{\mathrm{h}} \geq 8 \text {. Emission fre- } \\
\text { quency of } \mathrm{Li}^{8} \text { for diff. } \\
\text { intervals of } \mathrm{N}_{\mathrm{h}} \text {. Spect } \\
\text { of alphas from } \mathrm{Li} \text { decay }\end{array}$ & ira & - & $\begin{array}{l}\text { University of Warsaw; } \\
\text { Exposure Made at } \\
\text { Joint Institute for } \\
\text { Nuclear Research } \\
\text { Symchrocyclotron }\end{array}$ & $\begin{array}{l}\text { W. Gajewski et al., } \\
\text { Nuclear Phys: } 37 \\
226 \text { (1962) }\end{array}$ & \\
\hline 471 & $\mathrm{p}$ & 9 & $\begin{array}{l}\text { Heavy Emul- } \\
\text { sion Nuclei }\end{array}$ & $\begin{array}{l}\text { Charged distribu- } \\
\text { tion of fragments } \\
(4 \leq \ddot{Z} \leq 8)\end{array}$ & - & - & $\begin{array}{l}\text { Radium Institute, } \\
\text { USSR }\end{array}$ & $\begin{array}{l}\text { P.A. Gorichev, O.v. Lozhkin, } \\
\text { and N.A. Perfilov, Soviet } \\
\text { Phys.JETP } 41,27 \text { (1962) }\end{array}$ & \\
\hline 472 & $\mathrm{p}$ & 9.0 & $\begin{array}{l}\text { Heavy Em:l- } \\
\text { sion Nuclei }\end{array}$ & $\begin{array}{l}\sigma_{p}, f x \text { charge, } \\
\text { angle and energy } \\
\text { distribution of } \\
\text { fragments }\end{array}$ & $10-20$ & - & $\begin{array}{l}\text { Joint Institute } \\
\text { for Nuclear } \\
\text { Research, } \\
\text { Synchrotron }\end{array}$ & $\begin{array}{l}\text { N.A. Perfilov et al.; } \\
\text { Soviet Phys. -JETP 'I]; } \\
250 \text { (1960) }\end{array}$ & . \\
\hline 473 & $\mathrm{p}$ & 25 & $\begin{array}{l}\text { Heavy Emul- } \\
\text { sion Nuclei }\end{array}$ & $\begin{array}{l}\text { Angular and energy } \\
\text { distributions of } \\
\mathrm{Li}^{8} \text { and } L i^{\theta} \\
\text { fragments }\end{array}$ & - & - & $\begin{array}{l}\text { CERn, } \\
\text { Synchrotron }\end{array}$ & $\begin{array}{l}\text { G. Baumann, H. Braun, } \\
\text { and P. Cüer, Compt. } \\
\text { rend. 254 } 1966 \text { (1962) }\end{array}$ & \\
\hline 474 & $\mathrm{p}$ & 25 & $\begin{array}{l}\text { Heavy Houl- } \\
\text { sion Nuclei }\end{array}$ & $\begin{array}{l}\text { Energy spectra and } \\
\text { angular distribu- } \\
\text { tion of } \mathrm{Be}^{8} \text { fragments }\end{array}$ & - & - & - & $\begin{array}{l}\text { G. Baumann, H. Braun, } \\
\text { and P. Cüer, Compt. rend. } \\
\text { 254, } 2549 \text { (1962) }\end{array}$ & \\
\hline 475 & $p$ & 0.350 & $\begin{array}{l}\text { Emulsion } \\
\text { Nuclei }\end{array}$ & $\begin{array}{l}\text { Stars with fragments } \\
\text { vs. E E . No. of frag- } \\
\text { ments vs. charge. } \\
\text { Angular distribution } \\
\text { of fragments. Energy } \\
\text { distribution of fragmen }\end{array}$ & 20 & - & $\begin{array}{l}\text { Radium Institute; } \\
\text { Exposure Made at } \\
\text { Institute for Nuc- } \\
\text { lear Problems } \\
\text { Synchrocyclotron }\end{array}$ & $\begin{array}{l}\text { o.v. Lozhkin and N.A. } \\
\text { Perfilov; Soviet Phys:- } \\
\text { JETP 4, } 790 \text { (1957) }\end{array}$ & $\therefore$ \\
\hline
\end{tabular}




\begin{tabular}{|c|c|c|c|c|c|c|c|c|c|}
\hline No. & $\begin{array}{l}\text { Incident } \\
\text { Particle }\end{array}$ & $\begin{array}{l}\text { Incident } \\
\text { Particle } \\
\text { Energy (Gev) }\end{array}$ & Target & $\begin{array}{r}\text { Esti } \\
\text { Expe } \\
\text { Measured Quantity Err }\end{array}$ & $\begin{array}{l}\text { imate of } \\
\text { erimental } \\
\text { ror }(\%)\end{array}$ & Detector & $\begin{array}{l}\text { Installation and } \\
\text { Accelerator }\end{array}$ & References & Comments \\
\hline .476 & $p$ & 0.460 & $\begin{array}{l}\text { Emulsion } \\
\text { Nuslei }\end{array}$ & $\begin{array}{l}\text { Stars with fragments } \\
\text { vs. Ep. No. of frag- } \\
\text { ments vs. charge. } \\
\text { Angular distribution } \\
\text { of fragments. Energy } \\
\text { distribution of fragment }\end{array}$ & $\begin{array}{l}20 \\
\text { ts }\end{array}$ & - & $\begin{array}{l}\text { Radiur Institute; } \\
\text { Exposure Made at } \\
\text { Institute for } \\
\text { Nuclear Problems } \\
\text { Synchrocyclotron }\end{array}$ & $\begin{array}{l}\text { O.v. Lozhisin and N.A. } \\
\text { Perfilov, Soviet Phys.- } \\
\text { JETP 4, } 790 \text { (1957) }\end{array}$ & \\
\hline 477 & $\mathrm{p}$ & 0.560 & $\begin{array}{l}\text { Emulsion } \\
\text { Nuclei }\end{array}$ & $\begin{array}{l}\text { Stars with fragments } \\
\text { vs. } \mathrm{E}_{\mathrm{p}} \text {. No. of frag- } \\
\text { ments vs. charge. } \\
\text { Angular distribution of } \\
\text { fragments. Energy dis- } \\
\text { tributicn of fragments }\end{array}$ & 20 & - & $\begin{array}{l}\text { Radium Institute; } \\
\text { Exposure Made at } \\
\text { Institute for } \\
\text { Nuclear Problems } \\
\text { Synchrocyclotron }\end{array}$ & $\begin{array}{l}\text { O.V. Lozhlin and N.A. } \\
\text { Perfilov, Soviet Phys.- } \\
\text { JETP 4, } 790 \text { (1957) }\end{array}$ & \\
\hline 478 & $\mathrm{p}$ & 0.660 & $\begin{array}{l}\text { Emulsion } \\
\text { Nuclei }\end{array}$ & $\begin{array}{l}\text { Stars with fragments } \\
\text { vs. } \mathrm{E}_{\mathrm{p}} \text {. No. of frag- } \\
\text { ments vs. charge. } \\
\text { Angular distribution of } \\
\text { fragments. Energy dis- } \\
\text { tribution of fragnents }\end{array}$ & 20 & - & $\begin{array}{l}\text { Radium Institute; } \\
\text { Exposure Made at } \\
\text { Institute for } \\
\text { Nuclear Problems } \\
\text { Synchrocyclotron }\end{array}$ & $\begin{array}{l}\text { O.v. Lozhkin and N.A. } \\
\text { Perfilov, Soviet Phys.- } \\
\text { JETP 4, } 790 \text { (1957) }\end{array}$ & \\
\hline 479 & $\mathrm{p}$ & 0.660 & $\begin{array}{l}\text { Emulsion } \\
\text { Nuclei }\end{array}$ & $\begin{array}{l}\text { Prong distribution. } \\
\text { Angular distribution } \\
\text { of gray prongs, slow } \\
\text { protons, alphas. Energs } \\
\text { spectra of slow protons } \\
\text { and alphas. Yield of } \\
\text { charged } \pi^{\prime} s \text {. }\end{array}$ & - & - & $\begin{array}{l}\text { Institute for } \\
\text { Nuclear Problems }\end{array}$ & $\begin{array}{l}\text { I.P. Solov'eva, Soviet } \\
\text { Phys. JETP } 4,801 \text { (1957) }\end{array}$ & . \\
\hline 480 & $p$ & 0.925 & $\begin{array}{l}\text { Emulsion } \\
\text { Nuclei }\end{array}$ & $\begin{array}{l}\text { Momentum distribution } \\
\text { of bound nucleons }\end{array}$ & - & - & $\begin{array}{l}\text { Birmingham, } \\
\text { Symchrocyclotron }\end{array}$ & $\begin{array}{l}\text { J.G. McEwen, W.M. } \\
\text { Git son, and P.J. Duke, } \\
\text { Phil. Mag. 2, } 231 \text { (1957) }\end{array}$ & \\
\hline
\end{tabular}




\begin{tabular}{|c|c|c|c|c|c|c|c|c|c|}
\hline No. & $\begin{array}{l}\text { Incident } \\
\text { Particle }\end{array}$ & $\begin{array}{c}\text { Incident } \\
\text { Particle } \\
\text { Energy (Gev) }\end{array}$ & Target & Measured Quantity Ex & $\begin{array}{l}\text { Estimate of } \\
\text { Experimental } \\
\text { Error (\$) }\end{array}$ & Detector & $\begin{array}{l}\text { Installation and } \\
\text { Accelerator }\end{array}$ & References & Conments \\
\hline 481 & $\mathrm{p}$ & 5.7 & $\begin{array}{l}\text { Enulsion } \\
\text { Nuclei }\end{array}$ & $\begin{array}{l}\text { Mean number of pions } \\
\text { produced. Average } \\
\text { transverse momentum. } \\
\text { Shower and heavy } \\
\text { track distribution. } \\
\text { Angular distribution } \\
\text { of shower tracks }\end{array}$ & 15 & - & $\begin{array}{l}\text { Bristol; Exposure } \\
\text { Made at Berkeley } \\
\text { Bevatron }\end{array}$ & $\begin{array}{l}\text { V.Y. Rajopadhye, Phil. } \\
\text { Mag. } 5,537 \text { (1960) }\end{array}$ & . \\
\hline 482 & $p$ & 6 & $\begin{array}{l}\text { Emulsion } \\
\text { Nuclei }\end{array}$ & $\begin{array}{l}\text { Angular distribution } \\
\text { of secondary charged } \\
\pi \text { mesons }\end{array}$ & - & - & $\begin{array}{l}\text { Bristol; Exposure. } \\
\text { Made at Berkeley } \\
\text { Bevatron }\end{array}$ & $\begin{array}{l}\text { H.H. Aly and C.M. Fisher, } \\
\text { Nuovo cimento 17, } 98 \text {. } \\
\text { (1960) }\end{array}$ & \\
\hline 483 & $\mathrm{p}$ & $\begin{array}{l}6 \\
.\end{array}$ & $\begin{array}{l}\text { Enulsion } \\
\text { Inuclei }\end{array}$ & $\begin{array}{l}\text { Laboratory and C-M } \\
\text { system anguiar dis- } \\
\text { tribution of shower } \\
\text { tracks }\end{array}$ & - & - & $\begin{array}{l}\text { Poone, India; } \\
\text { Exposure Made at } \\
\text { Berkeley Bevatron }\end{array}$ & $\begin{array}{l}\text { V.Y. Rajopadhye, Proc. } \\
\text { Nat. Inst. Sci.' India A } \\
\text { 28, } 238 \text { (1962) }\end{array}$ & \\
\hline 484 & p. & 6.2 & $\begin{array}{l}\text { Erulsion } \\
\text { Nuclei }\end{array}$ & ${ }^{\sigma} p, x \pi y$ & 30 & - & $\begin{array}{l}\text { Phys. Inst., Bern; } \\
\text { Exposure Made at } \\
\text { Berkeley Bevatron }\end{array}$ & $\begin{array}{l}\text { H. Winzeler et al. } \\
\text { Nuovo cimento } 1 \frac{1}{7}, 8 \\
\text { (1960) }\end{array}$ & \\
\hline 485. & $\mathrm{p}$ & 8.7 & $\begin{array}{l}\text { Enulsion } \\
\text { Nuclei }\end{array}$ & $\begin{array}{l}\text { Angular distribution } \\
\text { of fast charged } \\
\text { particles from seconde } \\
\text { stars. Dis. of fast } \\
\text { particles from seconda } \\
\text { stars fomed by fast } \\
\text { neutrons }\end{array}$ & $\begin{array}{l}- \\
\text { daxy } \\
\text { dary }\end{array}$ & - & $\begin{array}{l}\text { Joint Institute } \\
\text { for Nuclear } \\
\text { Research, } \\
\text { Symchrotron }\end{array}$ & 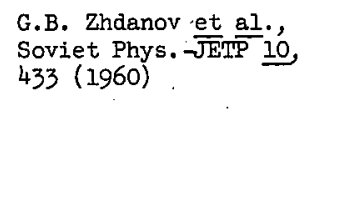 & . \\
\hline
\end{tabular}




\begin{tabular}{|c|c|c|c|c|c|c|c|c|c|}
\hline No. & $\begin{array}{l}\text { Incident } \\
\text { Particle }\end{array}$ & $\begin{array}{c}\text { Incident } \\
\text { Particle } \\
\text { Energy (Gev) }\end{array}$ & Target & Measured Quantity & $\begin{array}{l}\text { Estimate of } \\
\text { Experimental } \\
\text { Error }(\%)\end{array}$ & Detector & $\begin{array}{l}\text { Installation and } \\
\text { Accelerator }\end{array}$ & References & Comments \\
\hline 486 & $\mathrm{p}$ & 8.7 & $\begin{array}{l}\text { Imulsion } \\
\text { Nuclei }\end{array}$ & $\begin{array}{l}\text { Distribution for } \\
\text { various types of } \\
\text { fast particles } \\
\text { emitted. Integral } \\
\text { angular dis. of variou } \\
\text { types of emitted } \\
\text { particles, etc. }\end{array}$ & 25 & - & $\begin{array}{l}\text { Joint Institute } \\
\text { for Nuclear } \\
\text { Research, } \\
\text { Synchrotron }\end{array}$ & $\begin{array}{l}\text { G.B. Zhdanov et al., } \\
\text { Soviet Phys. JTETP } \\
442(1960)\end{array}$ & . \\
\hline $48 \%$ & $\mathrm{p}$. & $q$ & $\begin{array}{l}\text { Emulsion } \\
\text { Iucle: }\end{array}$ & $\begin{array}{l}\text { Mean energy of } \\
\pi^{0} \text { 's produced }\end{array}$ & 25 & - & $\begin{array}{l}\text { Joint Institute } \\
\text { for Nuclear } \\
\text { Research, } \\
\text { Symchrocyclotron }\end{array}$ & $\begin{array}{l}\text { G.I. Bayatyan et al., } \\
\text { Soviet Phys. JWTP } 2 \\
483 \text { (1959) }\end{array}$ & \\
\hline 488 & $\mathrm{p}$ & 9 & $\begin{array}{l}\text { Emulsion } \\
\text { Nuclei }\end{array}$ & $\begin{array}{l}\text { Distribution of } \\
\text { fast, and gray plus } \\
\text { black tracks. Angular } \\
\text { distribution of fast } \\
\text { particles }\end{array}$ & - & - & $\begin{array}{l}\text { Joint Institute } \\
\text { for Nuclear } \\
\text { Research, } \\
\text { Synchrocyclotron }\end{array}$ & $\begin{array}{l}\text { N.P. Bogachev et al., J: } \\
\text { Nuclear Energy } \frac{\text { al }}{178} \\
\text { (1959) }\end{array}$ & \\
\hline 490 & $p$ & 9 & $\begin{array}{l}\text { Emulsion } \\
\text { Nuelei }\end{array}$ & $\begin{array}{l}\text { Energy and angular } \\
\text { distribution of } \\
\text { high-energy alpha } \\
\text { particle }\end{array}$ & $\sim 30$ & - & $\begin{array}{l}\text { Kazakh State University; } \\
\text { Exposure Made at Joint } \\
\text { Insti ute for Nuclear } \\
\text { Research Synchrotron }\end{array}$ & $\begin{array}{l}\text { Zh. Takibaev, 'E. Shalagina, } \\
\text { end G. Tsadikova, Soviet } \\
\text { Fhys. Doklady 6, 1094. } \\
\text { (1962) }\end{array}$ & \\
\hline
\end{tabular}




\begin{tabular}{|c|c|c|c|c|c|c|c|c|c|}
\hline No. & $\begin{array}{l}\text { Incident } \\
\text { Particle }\end{array}$ & $\begin{array}{c}\text { Incident } \\
\text { Particle } \\
\text { Erergy (Gev) }\end{array}$ & Target & Measured Quantity & $\begin{array}{l}\text { Estimate of } \\
\text { Experimental } \\
\text { Error }(\%)\end{array}$ & Detector & $\begin{array}{l}\text { Installation and } \\
\text { Accelerator }\end{array}$ & References & Corments \\
\hline 491 & $\mathrm{p}$ & 10 & $\begin{array}{l}\text { Enulsion } \\
\text { Nuclei }\end{array}$ & $\begin{array}{l}\text { Average star charac- } \\
\text { teristics wi th pion, } \\
\text { proton, deuteron, } \\
\text { triton secondaries a } \\
\text { average kinematic pr } \\
\text { erties of secondarie }\end{array}$ & $\begin{array}{l}- \\
\text { or } \\
\text { and } \\
\text { rop- } \\
\text { es }\end{array}$ & - & $\begin{array}{l}\text { Joint Institute } \\
\text { for Nuclear } \\
\text { Research, } \\
\text { Synchrotron }\end{array}$ & $\begin{array}{l}\text { E. Balea et al. } \\
\text { Nuovo cimento } \\
214 \text { (1962) }\end{array}$ & \\
\hline 492 & $\mathrm{p}$ & 14 & $\begin{array}{l}\text { Emulsion } \\
\text { Nuclei }\end{array}$ & $\begin{array}{l}\text { Number and angular } \\
\text { distribution of } \\
\text { shower particles }\end{array}$ & $\sim 5$ & - & $\begin{array}{l}\text { Brussels ; Plates } \\
\text { Exposed at CERN } \\
\text { Synchrotron }\end{array}$ & $\begin{array}{l}\text { C. Bricman et al., } \\
\text { Nuovo cimento } 20 \text {, } \\
1017 \text { (1961) }\end{array}$ & \\
\hline 493 & $\mathrm{p} \quad 28$ & $8 \mathrm{Gev} / \mathrm{c}$ & $\begin{array}{l}\text { Enulsion } \\
\text { Nuclei }\end{array}$ & $\begin{array}{l}\text { Mean free path, } \\
\text { number and angular } \\
\text { distribution of } \\
\text { shower particles }\end{array}$ & - & - & $\begin{array}{l}\text { University of } \\
\text { Buffalo; - Plates } \\
\text { Exposed at Brook- } \\
\text { haven Symchrotron }\end{array}$ & $\begin{array}{l}\text { P.L. Jain et al.; } \\
\text { Nuovo cimento } \\
859 \text { (1961) }\end{array}$ & \\
\hline 494 & $\mathrm{n}$ & 0.095 & D & $\sigma_{\mathrm{nT}}$ & $\approx 5$ & $\begin{array}{l}\text { Bi Fission } \\
\text { Chamber }\end{array}$ & $\begin{array}{l}\text { Berkeley, } \\
\text { Cyclotron }\end{array}$ & $\begin{array}{l}\text { J. DeJuren and N. } \\
\text { Knable, Phys. Rev. } \\
\text { T7 } 606 \text { (1950) }\end{array}$ & $\begin{array}{l}\text { Good and poor } \\
\text { geometry }\end{array}$ \\
\hline 495 & $\mathrm{n}$ & 0.352 & L & $\sigma_{n T}$ & 1. & $\begin{array}{l}\text { Telescope } \\
\text { for Proton } \\
\text { Recoils }\end{array}$ & Liverpool & $\begin{array}{l}\text { A. Ashmore et al.', } \\
\text { Proc. Phys. Soc. A(Iondon) } \\
70,745 \text { (1957) }\end{array}$ & Good geometry \\
\hline 496 & $\mathrm{n}$ & 0.590 & D & $\begin{array}{l}\text { Atterpt to show } \\
\pi^{0} \text { pyoduction } \\
\propto \mathrm{A}^{2} 3\end{array}$ & - & $\begin{array}{l}\text { Scintillation } \\
\text { Counter Tele- } \\
\text { scope and Cerenko } \\
\text { Detectors }\end{array}$ & $\begin{array}{l}\text { Institute for } \\
\text { Nuclear Problems, } \\
\text { ov } \\
\text { Synchrocyclotron }\end{array}$ & $\begin{array}{l}\text { V.P. Dzhelepov, K.O. } \\
\text { Oganesian, and V.B. } \\
\text { Fliagin, Soviet Phys.- } \\
\text { JETP 5, } 560 \text { (1957) }\end{array}$ & \\
\hline 497 & $\mathrm{n}$ & 0.765 & D & $\sigma_{n T}$ & 5 & $\begin{array}{l}\text { Perspex } \\
\text { Cerenkov } \\
\text { Detector }\end{array}$ & $\begin{array}{l}\text { Birmingham, } \\
\text { Synchrotron }\end{array}$ & $\begin{array}{l}\text { N.E. Booth, G.W. Hut- } \\
\text { chinson, and B. Ledley, } \\
\text { Proc. Phys. Soc. (London) } \\
\text { 1, } 293 \text { (1958) }\end{array}$ & $\begin{array}{l}\text { Good and poor } \\
\text { Geometry }\end{array}$ \\
\hline
\end{tabular}




\begin{tabular}{|c|c|c|c|c|c|c|c|c|c|}
\hline No. & $\begin{array}{l}\text { Incident } \\
\text { Farticle }\end{array}$ & $\begin{array}{c}\text { Incident } \\
\text { Particle } \\
\text { Energy (Gev). }\end{array}$ & Target & Measured Quantity & $\begin{array}{l}\text { Estimate of } \\
\text { Experimental } \\
\text { Error }(\%)\end{array}$ & Detector & $\begin{array}{l}\text { Installation and } \\
\text { Accelerator }\end{array}$ & References & Comments \\
\hline 498 & $\mathrm{n}$ & 0.765 & $\mathrm{Li}$ & $\sigma_{n T}$ & 5 & $\begin{array}{l}\text { Perspex } \\
\text { Cerenkov } \\
\text { Detector }\end{array}$ & $\begin{array}{l}\text { Birmingham, } \\
\text { Synchrotron }\end{array}$ & $\begin{array}{l}\text { N.E. Bocth, G.W. Hut- } \\
\text { chinson, and B. Ledley, } \\
\text { Proc. Pbys. Soc. (London) } \\
\text { T1, } 293 \text { (1958) }\end{array}$ & $\begin{array}{l}\text { Good and poor } \\
\text { geometry }\end{array}$ \\
\hline 499 & $\mathrm{n}$ & 0.095 & $\mathrm{Be}$ & $\sigma_{n T}$ & $\approx 5$ & $\begin{array}{l}\text { Bi Fission } \\
\text { Chamber }\end{array}$ & $\begin{array}{l}\text { Berkeley, } \\
\text { Cyclotron }\end{array}$ & $\begin{array}{l}\text { J. DeJuren and N. Knable, } \\
\text { Phys. Rev. 77, } 606 \text { (1950) }\end{array}$ & $\begin{array}{l}\text { Good and poor } \\
\text { geometry }\end{array}$ \\
\hline 500 & $\mathrm{n}$ & 0.590 & $\mathrm{Be}$ & $\begin{array}{l}\text { Attempt to show } \\
\pi^{0} \text { pyoduction } \\
\propto \mathrm{A}^{2 / 3}\end{array}$ & - & $\begin{array}{l}\text { Scintillation } \\
\text { Counter Tele- } \\
\text { scopes and Ceren- } \\
\text { kov Detectors }\end{array}$ & $\begin{array}{l}\text { Institute for } \\
\text { Nuclear Problems, } \\
\text { - Synchrocyclotron }\end{array}$ & $\begin{array}{l}\text { V.P. Dzhelepov, K.O. } \\
\text { Oganesian, and V.B. } \\
\text { Fliagin, Soviet Phys.- } \\
\text { JETP 5, } 560 \text { (1957) }\end{array}$ & \\
\hline 501 & $n$ & 0.550 & $\mathrm{Be}$ & $\begin{array}{l}\sigma_{\mathrm{nT}} \\
\sigma_{\mathrm{nX}}\end{array}$ & 5 & $\begin{array}{l}\text { Telescope, } \\
\text { Liquic } \\
\text { Scintillator }\end{array}$ & $\begin{array}{l}\text { Insti.tute for } \\
\text { Nuclear Problems, } \\
\text { Synchrocyclotron }\end{array}$ & $\begin{array}{l}\text { V.I. Moskalev and B.V. } \\
\text { Gavrilovskii, Soviet } \\
\text { Phys. Dollady 1, } 607 \\
\text { (1956) }\end{array}$ & $\begin{array}{l}\text { Good and poor } \\
\text { geometry }\end{array}$ \\
\hline 502 & $n$ & 0.860 & $\mathrm{Be}$ & $\begin{array}{l}\sigma_{\mathrm{nT}} \\
\sigma_{\mathrm{nX}}\end{array}$ & $5-10$ & $\begin{array}{l}\text { Counter } \\
\text { Telescope }\end{array}$ & $\begin{array}{l}\text { Brookhaven, } \\
\text { Cosmotron }\end{array}$ & $\begin{array}{l}\text { F.F. Chen, C.P. Leavitt, } \\
\text { and A.M. Shapiro, Phys. } \\
\text { Rev. 29, } 857 \text { (1955) }\end{array}$ & $\begin{array}{l}\text { Good and poor } \\
\text { geometry }\end{array}$ \\
\hline 503 & $\mathrm{n}$ & 1.4 & $\mathrm{Be}$ & $\begin{array}{l}\sigma_{n T} \\
\sigma_{n X}\end{array}$ & $3-5$ & $\begin{array}{l}\text { Al Radiator } \\
\text { and Scintilla- } \\
\text { tion Counter } \\
\text { Telescope }\end{array}$ & $\begin{array}{l}\text { Brookhaven, } \\
\text { Cosmotron }\end{array}$ & $\begin{array}{l}\text { T. Coor } \frac{\in \mathrm{t}}{13} \text { al., Phys. } \\
\text { Rev. } 98,1955)\end{array}$ & $\begin{array}{l}\text { Good and poor } \\
\text { geometry }\end{array}$ \\
\hline 504 & $\mathrm{n}$ & 0.090 & $\mathrm{c}$ & 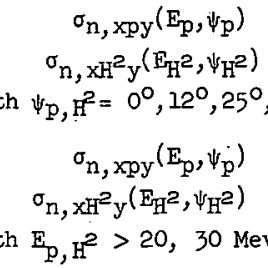 & $45^{\circ}$ & $\begin{array}{l}\text { Ho and Specifie } \\
\text { Ionization } \\
\text { Telescope }\end{array}$ & $\begin{array}{l}\text { Berkeley, } \\
\text { Synchrocyclotron }\end{array}$ & $\begin{array}{l}\text { J. Hadley and H. York, } \\
\text { Phys. Rev. } 80,345 \\
\text { (1950) }\end{array}$ & \\
\hline
\end{tabular}




\begin{tabular}{|c|c|c|c|c|c|c|c|c|c|}
\hline No. & $\begin{array}{l}\text { Incident } \\
\text { Particle }\end{array}$ & $\begin{array}{l}\text { Incident } \\
\text { Particle } \\
\text { Energy (Gev) }\end{array}$ & Target & Measured Quantity & $\begin{array}{l}\text { Estimate of } \\
\text { Experimental } \\
\text { Error }(\phi)\end{array}$ & Detector & $\begin{array}{l}\text { Installation and } \\
\text { Accelerator }\end{array}$ & References & Comments \\
\hline 505 & $\mathrm{n}$ & 0.095 & $\mathrm{C}$ & $\sigma_{n T}, \quad \sigma_{n X}$ & $\approx 5$ & $\begin{array}{l}\text { Bi Fission } \\
\text { Chamber }\end{array}$ & $\begin{array}{l}\text { Berkeley, } \\
\text { Cyclotron }\end{array}$ & $\begin{array}{l}\text { J. DeJuren and N. Knable, } \\
\text { Phys. Rev. 77, } 606 \text { (1950) }\end{array}$ & $\begin{array}{l}\text { Good and poor } \\
\text { geometry }\end{array}$ \\
\hline 506 & $\mathrm{n}$ & -0.130 & $\mathrm{c}$ & $\begin{array}{l}\sigma_{\mathrm{n}, \ln l p} \text { for vari- } \\
\text { ous excited final } \\
\text { states }\end{array}$ & $20-30$ & $\begin{array}{l}\text { Counter Tele- } \\
\text { scope and NaI } \\
\text { Crystal }\end{array}$ & $\begin{array}{l}\text { Oxford, Atomic } \\
\text { Energy Research } \\
\text { Establishment, } \\
\text { Cyclotron }\end{array}$ & $\begin{array}{l}\text { S.M. Austin et } \frac{\text { al. }}{38} \text {, Proc. } \\
\text { Phys. Soc. } \frac{80}{383} \\
\text { (1962) }\end{array}$ & \\
\hline 507 & $\mathrm{n}$ & 0.140 & $\mathrm{C}^{12}$ & $\begin{array}{l}\text { Excited state } \\
\text { cross sections } \\
\text { in }(n, n g) \\
\text { reactions }\end{array}$ & $20-50$ & $\begin{array}{l}\text { Coincidence } \\
\text { Counters and } \\
\text { NaI Crystals }\end{array}$ & $\begin{array}{l}\text { Harwell, } \\
\text { Cyclotron }\end{array}$ & $\begin{array}{l}\text { S. Austin et al., } \\
\text { Rutherford Jubilee Conf. } \\
\text { Manchester } 1961 \\
\text { Paper C/I } 139\end{array}$ & Poor geometry \\
\hline 508 & $\mathrm{n}$ & $0.034-0.300$ & $\mathrm{c}$ & $\sigma_{n X}$ & $3-15$ & - & - & $\begin{array}{l}\text { G.P. Millburn et al., } \\
\text { Phys. Rev. } 95, \frac{\text { al }}{1268} \\
\text { (1954) }\end{array}$ & A compilation \\
\hline 509 & $\mathrm{n}$ & 0.352 & C & $\sigma_{n T}$ & 1 & $\begin{array}{l}\text { Telescope for } \\
\text { Proton Recoils }\end{array}$ & Liverpool & $\begin{array}{l}\text { A. Ashmore et al., Proc. } \\
\text { Phys. Soc. A(Iondon) } \\
70,745 \text { (1957). }\end{array}$ & Good geometry \\
\hline 510 & $\mathrm{n}$ & 0.590 & $\mathrm{c}$ & $\begin{array}{l}\text { Attempt to show } \\
\pi^{\circ} \text { prgduction } \\
\alpha \mathrm{A}^{2}\end{array}$ & - & $\begin{array}{l}\text { Scintillation } \\
\text { Counter Tele- } \\
\text { scope and Ceren- } \\
\text { kov Counters }\end{array}$ & $\begin{array}{l}\text { Institute for } \\
\text { Nuclear Problems, } \\
\text { Synchrocyclotron }\end{array}$ & $\begin{array}{l}\text { V.P. Dzhelepov, K.O. } \\
\text { Oganesian, and V.B. } \\
\text { Fliagin, Soviet Phys.- } \\
\text { JETP } 5 \text {, } 560 \text { (1957) }\end{array}$ & \\
\hline 511 & $\mathrm{n}$ & 0.650 & $\mathrm{c}$ & $\begin{array}{l}\sigma_{\mathrm{nT}} \\
\sigma_{\mathrm{nX}}\end{array}$ & 5 & $\begin{array}{l}\text { Telescope, } \\
\text { Liquid } \\
\text { Scir:tillator }\end{array}$ & $\begin{array}{l}\text { Institute for } \\
\text { Nuclear Problems, } \\
\text { Synchrocyclotron }\end{array}$ & $\begin{array}{l}\text { V.I. Moskalev and B.V. } \\
\text { Gavrilovski1, Soviet Phys. } \\
\text { Doklady } 1,607 \text { (1956) }\end{array}$ & $\begin{array}{l}\text { Good and poor } \\
\text { geometry }\end{array}$ \\
\hline
\end{tabular}




\begin{tabular}{|c|c|c|c|c|c|c|c|c|c|}
\hline No. & $\begin{array}{l}\text { Incident } \\
\text { Particle }\end{array}$ & $\begin{array}{c}\text { Incident } \\
\text { Particle } \\
\text { Energy (Gev) }\end{array}$ & Target & Measured Quantity & $\begin{array}{l}\text { Estimate of } \\
\text { Experimental } \\
\text { Error }(\%)\end{array}$ & Detector & $\begin{array}{l}\text { Installation and } \\
\text { Accelerator }\end{array}$ & References & Comments \\
\hline 512 & $n$ & 0.765 & $\mathrm{c}$ & $\sigma_{n T}, \sigma_{n X}$ & 5 & $\begin{array}{l}\text { Perspex } \\
\text { Cerenisov } \\
\text { Detector }\end{array}$ & $\begin{array}{l}\text { Birmingham, } \\
\text { Synchrotron }\end{array}$ & $\begin{array}{l}\text { N.E. Booth, G.W. Hut- } \\
\text { chinson, and B. Ledley, } \\
\text { Proc. Phys. Soc. (London) } \\
\text { 11, } 293 \text { (1958) }\end{array}$ & $\begin{array}{l}\text { Good and poor } \\
\text { geometry }\end{array}$ \\
\hline 513 & $n$ & 0.860 & $\mathrm{c}$ & $\sigma_{n T}: s_{n X}$ & $5-10$ & $\begin{array}{l}\text { Counter } \\
\text { Telescope }\end{array}$ & $\begin{array}{l}\text { Brookhaven, } \\
\text { Cosmotron }\end{array}$ & $\begin{array}{l}\text { F.F. Chen, C.P. Leavitt, } \\
\text { and A.M. Shapiro, Phys. } \\
\text { Rev. } 99,857 \text { (1955) }\end{array}$ & $\begin{array}{l}\text { Good and poor } \\
\text { geometry }\end{array}$ \\
\hline 514 & $\mathrm{n}$ & 1.4 & $\mathrm{c}$ & $\sigma_{n T}, \sigma_{n X}$ & $3-5$ & $\begin{array}{l}\text { Al Radiator and } \\
\text { Scintillation } \\
\text { Counter Telescope }\end{array}$ & $\begin{array}{l}\text { Brookhaven, } \\
\text { Cosmotron }\end{array}$ & $\begin{array}{l}\text { T. Coor et al.', Phys. } \\
\text { Rev. } 98, \frac{1}{13} \frac{1955)}{(1955)}\end{array}$ & $\begin{array}{l}\text { Good and poor } \\
\text { geometry }\end{array}$ \\
\hline 515 & $\mathrm{n}$ & 4.5 & c & $\sigma_{n T}, \sigma_{n X}$ & $\sim 5$ & $\begin{array}{l}\text { Counter } \\
\text { Telescope }\end{array}$ & $\begin{array}{l}\text { Berkeley, } \\
\text { Bevatron }\end{array}$ & $\begin{array}{l}\text { J.H. Atkinson et al., } \\
\text { Phys. Rev. Letters 2, } \\
168 \text { (1959) }\end{array}$ & $\begin{array}{l}\text { Good and poor } \\
\text { geometry }\end{array}$ \\
\hline 516 & $\mathrm{n}$ & 0.095 & $\mathrm{~N}$ & $\sigma_{n T}$ & $\approx 5$ & $\begin{array}{l}\text { Bi Fission } \\
\text { Chamber }\end{array}$ & $\begin{array}{l}\text { Bèrkeley, } \\
\text { Cyclotron }\end{array}$ & $\begin{array}{l}\text { J. DeJuren and N. Knable, } \\
\text { Phys. Rev. 77; } 606 \text { (1950) }\end{array}$ & $\begin{array}{l}\text { Good and poor } \\
\text { geometry }\end{array}$ \\
\hline 517 & $\mathrm{n}$ & 0.095 & 0 & $\sigma_{n T}$ & $\approx 5$ & $\begin{array}{l}\text { Bi Fission } \\
\text { Chamber }\end{array}$ & $\begin{array}{l}\text { Bexkeley, } \\
\text { Cyclotron }\end{array}$ & $\begin{array}{l}\text { J. DeJuren and N. Knable, } \\
\text { Phys. Rev. 77, } 606 \text { (1950) }\end{array}$ & $\begin{array}{l}\text { Good and poor } \\
\text { geometry }\end{array}$ \\
\hline 518 & $\mathrm{n}$ & 0.352 & 0 & $\sigma_{n T}$ & 1 & $\begin{array}{l}\text { Telescope for } \\
\text { Proton Recoils }\end{array}$ & Liverpool & $\begin{array}{l}\text { A. Ashmore et al., Proc. } \\
\text { Phys. Soc. A(London) } \\
70,745 \text { (1957) }\end{array}$ & Good geometry \\
\hline 519 & $n$ & 0.590 & 0 & $\begin{array}{l}\text { Attempt to show } \\
\pi^{0} \text { production } \\
\left.\propto A^{2}\right\}_{3}\end{array}$ & - & $\begin{array}{lr}\text { Scintillation } & \text { Ir } \\
\text { Counter Tele- } & \mathrm{N} \\
\text { scope and } & \mathrm{S}_{3} \\
\text { Cerenkov Detec tor }\end{array}$ & $\begin{array}{l}\text { Institute for } \\
\text { Nuclear Problems, } \\
\text { Synchrocyclotron } \\
\text { r }\end{array}$ & $\begin{array}{l}\text { V.P. Dzhelepov, K.O. } \\
\text { Oganesian, and V.B. } \\
\text { Fliagin, Soviet Phys.- } \\
\text { JETP 5, } 560 \text { (1957) }\end{array}$ & \\
\hline
\end{tabular}




\begin{tabular}{|c|c|c|c|c|c|c|c|c|c|}
\hline No. & $\begin{array}{l}\text { Incident } \\
\text { Particle }\end{array}$ & $\begin{array}{c}\text { Incident } \\
\text { Particle } \\
\text { Energy (Gev) }\end{array}$ & Target & Measured Quantity & $\begin{array}{l}\text { Estimate of } \\
\text { Experimental } \\
\text { Error }(\%)\end{array}$ & Detector & $\begin{array}{l}\text { Installation and } \\
\text { Accelerator }\end{array}$ & References & Conments \\
\hline 520 & $\mathrm{n}$ & 0.650 & 0 & $\sigma_{\mathrm{nT}}, \quad \sigma_{\mathrm{nX}}$ & 5 & $\begin{array}{l}\text { Telescope, } \\
\text { Liquid } \\
\text { Scintillator }\end{array}$ & $\begin{array}{l}\text { Institute for } \\
\text { Nuclear Problems, } \\
\text { Synchrocyclotron }\end{array}$ & $\begin{array}{l}\text { V.I. Moskalev and B.V. } \\
\text { Gavrilovskii, Soviet } \\
\text { Phys. Doklady } 1,607 \\
\text { (1956) }\end{array}$ & $\begin{array}{l}\text { Good and poor. } \\
\text { geometry }\end{array}$ \\
\hline 521 & $\mathrm{n}$ & 0.765 & 0 & $\sigma_{n T}$ & 5 & $\begin{array}{l}\text { Perspex } \\
\text { Cerenkov } \\
\text { Detector }\end{array}$ & $\begin{array}{l}\text { Birminghem, } \\
\text { Synchrotron }\end{array}$ & $\begin{array}{l}\text { N.E. Booth, G.W. Hut- } \\
\text { chinson, and B. Ledley; } \\
\text { Proc. Phys. Soc. (London) } \\
\text { II, } 293 \text { (1958) }\end{array}$ & $\begin{array}{l}\text { Good and poor } \\
\text { geometry }\end{array}$ \\
\hline 522 & $\mathrm{n}$ & 0.090 & Al & Neutron yield & 10 & $\begin{array}{l}\mathrm{MnSO}_{4} \\
\text { Solution }\end{array}$ & $\begin{array}{l}\text { Berkeley and } \\
\text { Chicago Cyclotrons }\end{array}$ & $\begin{array}{l}\text { W.E. Crandall and G.P. } \\
\text { Millburn, J. Appl. Phys. } \\
\text { 29 } 698(1958)\end{array}$ & \\
\hline 523 & $\mathrm{n}$ & 0.095 & Al & $\sigma_{n T}, \sigma_{n X}$ & $\approx 5$ & $\begin{array}{l}\text { Bi Fission } \\
\text { Chamber }\end{array}$ & $\begin{array}{l}\text { Berkeley, } \\
\text { Cyclotron }\end{array}$ & $\begin{array}{l}\text { J. DeJuren and N. Knable, } \\
\text { Phys. Rev. 77, } 606 \text { (1950) }\end{array}$ & $\begin{array}{l}\text { Good and poor } \\
\text { geometry. }\end{array}$ \\
\hline 524 & $\mathrm{n}$ & $0.084-0.300$ & $\mathrm{AI}$ & $\sigma_{n x}$ & $3-15$ & - & - & $\begin{array}{l}\text { G.P. Millburn et al., Phys. } \\
\text { Rev. } 25,1268 \text { (1954) }\end{array}$ & A compilation \\
\hline 525 & $\mathrm{n}$ & 0.352 & Al & $\sigma_{n T}$ & 1 & $\begin{array}{l}\text { Telescope for } \\
\text { Proton Recoils }\end{array}$ & Liverpool & $\begin{array}{l}\text { A. Ashroore et al., Proc. } \\
\text { Phys. Soc. A(London)' } \\
\text { 70 } 745 \text { (1957) }\end{array}$ & Good geometry \\
\hline 526 & $\mathrm{n}$ & 0.590 & $\mathrm{Al}$ & $\begin{array}{l}\text { Attempt to show } \\
\pi^{0} \text { prgduction } \\
\propto \mathrm{A}^{2}\end{array}$ & - & $\begin{array}{l}\text { Scirtillation } \\
\text { Courter Tele- } \\
\text { scope and } \\
\text { Cerenkov Detecto }\end{array}$ & $\begin{array}{l}\text { Institute for } \\
\text { Nuclear Problems, } \\
\text { Synchrocyclotron } \\
\text { or }\end{array}$ & $\begin{array}{l}\text { V.P. Dzhelepov, K.O. } \\
\text { Oganesian, and V.P. } \\
\text { Fliagin, Soviet Phys.- } \\
\text { JETP 5, } 560 \text { (1957) }\end{array}$ & \\
\hline
\end{tabular}




\begin{tabular}{|c|c|c|c|c|c|c|c|c|c|}
\hline No. & $\begin{array}{l}\text { Incident } \\
\text { Particle }\end{array}$ & $\begin{array}{l}\text { Incident } \\
\text { Particle } \\
\text { Energy (Gev) }\end{array}$ & Tanget & Measured Quantity & $\begin{array}{l}\text { Estimate of } \\
\text { Experimental } \\
\text { Error }(\phi)\end{array}$ & Detector & $\begin{array}{l}\text { Installation and } \\
\text { Accelerator }\end{array}$ & References & Comments \\
\hline 527 & $\mathrm{n}$ & 0.650 & Al & $\sigma_{n T}, \sigma_{n X}$ & 5 & $\begin{array}{l}\text { Telescope, } \\
\text { Liquid } \\
\text { Scintillator }\end{array}$ & $\begin{array}{l}\text { Institute for } \\
\text { Nuclear Problems, } \\
\text { Synchrocyclotron }\end{array}$ & $\begin{array}{l}\text { V.I. Moskalev and B.V. } \\
\text { Gavrilovskii, Soviet Phys. } \\
\text { Doklady } 1,607 \text { (1956) }\end{array}$ & $\begin{array}{l}\text { Good and poor } \\
\text { geometry }\end{array}$ \\
\hline 528 & $\mathrm{n}$ & 0.765 & AII & $\sigma_{n T}, \sigma_{n X}$ & 5 & $\begin{array}{l}\text { Perspex } \\
\text { Cerenkov } \\
\text { Detector }\end{array}$ & $\begin{array}{l}\text { Birmingham, } \\
\text { Synchrotron }\end{array}$ & $\begin{array}{l}\text { N.E. Booth, G.W. Hut- } \\
\text { chinson, and B. Ledley, } \\
\text { Proc. Phys. Soc. (London) } \\
71,293 \text { (1958) }\end{array}$ & $\begin{array}{l}\text { Good and poor } \\
\text { geometry }\end{array}$ \\
\hline $5 \approx 9$ & $\mathrm{n}$ & 0.860 & $A .1$ & $\sigma_{\mathrm{nT}}, \sigma_{\mathrm{nX}}$ & $5-10$ & $\begin{array}{l}\text { Counter } \\
\text { Telescope }\end{array}$ & $\begin{array}{l}\text { Brookhaven, } \\
\text { Cosmotron }\end{array}$ & $\begin{array}{l}\text { F.F. Chen, C.P. Leavitt, } \\
\text { and A.M. Shapiro, Phys.' } \\
\text { Rev. 99, } 857 \text { (1955) }\end{array}$ & $\begin{array}{l}\text { Good and poor } \\
\text { geometry }\end{array}$ \\
\hline 530 & $\mathrm{n}$ & 1.4 & $\mathrm{Al}$ & $\sigma_{n T}, \quad \sigma_{n X}$ & $3-5$ & $\begin{array}{l}\text { Al Radiator } \\
\text { and Scintilla- } \\
\text { tion Counter } \\
\text { Telescope }\end{array}$ & $\begin{array}{l}\text { Brookhaven, } \\
\text { Cosmotron }\end{array}$ & $\begin{array}{l}\text { T. Coor et al; Phys. } \\
\text { Rev. } 98 \text { 1369 (1955) }\end{array}$ & $\begin{array}{l}\text { Good and poor } \\
\text { geometry }\end{array}$ \\
\hline 531 & $\dot{\mathrm{n}}$ & 3.6 & A] & ${ }^{\sigma} \mathrm{nX}$ & $\sim 15$ & $\begin{array}{l}\text { Star Density } \\
\text { in Nuclear } \\
\text { Emulsions }\end{array}$ & $\begin{array}{l}\text { University of Cal., } \\
\text { Santa Barbara, } \\
\text { Bevatron }\end{array}$ & $\begin{array}{l}\text { P.H. Barrett, Phys. Rev. } \\
\text { I14, } 1374 \text { (1959) }\end{array}$ & Poor geometry \\
\hline 532 & $\mathrm{n}$ & 0.095 & $\mathrm{Cl}$ & $\sigma_{n T}$ & $\approx 5$ & $\begin{array}{l}\text { Bi Fission } \\
\text { Chamber }\end{array}$ & $\begin{array}{l}\text { Berkeley, } \\
\text { Cyclotron }\end{array}$ & $\begin{array}{l}\text { J. DeJuren and N. Knable, } \\
\text { Phys. Rev. T7, } 606(1950)\end{array}$ & $\begin{array}{l}\text { Good and poor } \\
\text { geometry }\end{array}$ \\
\hline 533 & $\mathrm{n}$ & 0.090 & $\mathrm{Cu}$ & Neutron yield & 10 & $\begin{array}{l}\mathrm{MnSO}_{4} \\
\text { Solution }\end{array}$ & $\begin{array}{l}\text { Berkeley and } \\
\text { Chicago Cyclotrons }\end{array}$ & $\begin{array}{l}\text { W.E. Crandall and G.P. } \\
\text { Millburn, J. Appl. Phys. } \\
\text { 29, } 698 \text { (1958) }\end{array}$ & \\
\hline
\end{tabular}




\begin{tabular}{|c|c|c|c|c|c|c|c|c|c|}
\hline No. & $\begin{array}{l}\text { Incident } \\
\text { Particle }\end{array}$ & $\begin{array}{cc}\text { Incident } \\
\text { Particle } \\
\text { Energy (Gev) } \\
\end{array}$ & Target & Measured Quantity & $\begin{array}{l}\text { Estimate of } \\
\text { Experimental } \\
\text { Error }(\phi)\end{array}$ & Detector & $\begin{array}{l}\text { Installation and } \\
\text { Accelerator }\end{array}$ & References & Comments \\
\hline 534 & $\dot{n}$ & 0.090 & $\mathrm{C}-x$ & $\begin{array}{c}\sigma_{\mathrm{n}, \mathrm{xpy}}\left(E_{\mathrm{p}}, \psi_{\mathrm{p}}\right) \\
\sigma_{\mathrm{n}, \mathrm{xH}} \mathrm{H}^{2}\left(\mathrm{E}_{\mathrm{H}}{ }^{2}, \psi_{\mathrm{H}^{2}}\right) \\
\mathrm{h} \psi_{\mathrm{p}, \mathrm{H}^{2}}=0^{0}, 12^{\circ}, 25^{\circ} \\
\sigma_{\mathrm{n}, \mathrm{xpy}}\left(E_{\mathrm{p}}, \psi_{\mathrm{p}}\right) \\
\sigma_{\mathrm{n}, \mathrm{xH}^{2} \mathrm{y}}\left(\mathrm{E}_{\mathrm{H}}{ }^{2}, \psi_{\mathrm{H}}{ }^{2}\right) \\
\mathrm{h} \mathrm{E}_{\mathrm{p}, \mathrm{H}^{2}}>20,30 \mathrm{Mev}\end{array}$ & $45^{\circ}$ & $\begin{array}{l}\text { Hp and Specific } \\
\text { Ionization } \\
\text { Telescope }\end{array}$ & $\begin{array}{c}\text { Berkeley, } \\
\text { Synchrocyclotron }\end{array}$ & $\begin{array}{l}\text { J. Fadley and H. York, } \\
\text { Phys. Rev. } 80,345 \\
\text { (1950) }\end{array}$ & . \\
\hline 535 & $\mathrm{n}$ & 0.095 & Cu & $\sigma_{n T}, \sigma_{n X}$ & $\underset{1}{\leftarrow} 5$ & $\begin{array}{l}\text { Bi Fission } \\
\text { Chamber }\end{array}$ & $\begin{array}{l}\text { Berkeley, } \\
\text { Cyclotron }\end{array}$ & $\begin{array}{l}\text { J. DeJuren and N. Knable, } \\
\text { Phys. Rev. } 77,606(1950)\end{array}$ & $\begin{array}{l}\text { Good and poor } \\
\text { geometry }\end{array}$ \\
\hline 536 & $\mathrm{n}$ & $0.084-0.300$ & $\mathrm{Cu}$ & $\sigma_{n X}$ & $3-15$ & - & - & $\begin{array}{l}\text { G.P. Millburn et al.' } \\
\text { Phys. Rev. } 95,1268 \text { (1954) }\end{array}$ & A compilation \\
\hline 537 & $\mathrm{n}$ & 0.352 & $\mathrm{Cu}$ & $\sigma_{n T}$ & 1 & $\begin{array}{l}\text { Telescope for } \\
\text { Proton Recoils }\end{array}$ & Liverpool & $\begin{array}{l}\text { A. Ashmore et al., Proc. } \\
\text { Phys. Soc. A(London) } \\
\text { 0, } 745 \text { (1957) }\end{array}$ & Good geometry \\
\hline 538 & $\mathrm{n}$ & 0.590 & $\mathrm{Cu}$ & $\begin{array}{l}\text { Attermpt to show } \\
\pi^{0} \text { prgduction } \\
\propto \mathrm{A}^{2}{ }_{3}\end{array}$ & - & $\begin{array}{l}\text { Scintillation } \\
\text { Counter Teles- } \\
\text { cope and } \\
\text { Cerenkov Detecto }\end{array}$ & $\begin{array}{l}\text { Institute for } \\
\text { Nuclear Problems, } \\
\text { Synchrocyclotron } \\
\text { or }\end{array}$ & $\begin{array}{l}\text { V.P. Dzhelepov, K.0. } \\
\text { Oganesian, and V.B. } \\
\text { Fliagin, Soviet Phys.- } \\
\text { JETP-5, } 560 \text { (1957) }\end{array}$ & 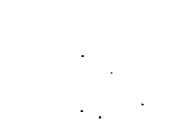 \\
\hline 535 & $\mathrm{n}$ & 0.650 & $\mathrm{Cu}$ & $\sigma_{n \mathrm{~T}}, \sigma_{\mathrm{nX}}$ & 5 & $\begin{array}{l}\text { Telescope, } \\
\text { Liquid } \\
\text { Scintillator }\end{array}$ & $\begin{array}{l}\text { Institute for } \\
\text { Nuclear Problems, } \\
\text { Synchrocyclotron }\end{array}$ & $\begin{array}{l}\text { V.I. Moskalev and B.V. } \\
\text { Gavriloviskii, Soviet } \\
\text { Phys. Doklady I, } 607 \\
\text { (1956) }\end{array}$ & $\begin{array}{l}\text { Good and poor } \\
\text { geometry }\end{array}$ \\
\hline 540 & $\mathrm{n}$ & 0.765 & $\mathrm{Cu}$ & $\sigma_{\mathrm{nT}} ; \sigma_{\mathrm{nX}}$ & 5 & $\begin{array}{l}\text { Perspex } \\
\text { Cerenkov } \\
\text { Detector }\end{array}$ & $\begin{array}{l}\text { Birmingham, } \\
\text { Synchrotron }\end{array}$ & $\begin{array}{l}\text { N.E. Booth, G.W. Hut- } \\
\text { chinson, and B. Ledley, } \\
\text { Proc. Phys. Soc. (London) } \\
\text { I1, } 293 \text { (1958) }\end{array}$ & $\begin{array}{l}\text { Good and poor } \\
\text { geometry }\end{array}$ \\
\hline
\end{tabular}




\begin{tabular}{|c|c|c|c|c|c|c|c|c|c|}
\hline No. & $\begin{array}{l}\text { Incident } \\
\text { Particle }\end{array}$ & $\begin{array}{l}\text { Incilent } \\
\text { Particle } \\
\text { Energy (Gev) }\end{array}$ & Target & Measured Quantity & $\begin{array}{l}\text { Estimate of } \\
\text { Experimental } \\
\text { Error }(\%)\end{array}$ & Detecto: & $\begin{array}{l}\text { Installation and } \\
\text { Accelerator }\end{array}$ & References & Corments \\
\hline 541 & $\mathrm{n}$ & 0.860 & $\mathrm{Cr}$ & $\sigma_{n T}, a_{n X}$ & $5-10$ & $\begin{array}{l}\text { Counter } \\
\text { Telescope }\end{array}$ & $\begin{array}{l}\text { Brookhaven, } \\
\text { Cosmotron }\end{array}$ & $\begin{array}{l}\text { F.F. Chen, C.P. Leavitt, } \\
\text { and A.M. Shapiro, Phys. } \\
\text { Rev. 99, } 857 \text { (1955) }\end{array}$ & $\begin{array}{l}\text { Good and poor } \\
\text { Geometry }\end{array}$ \\
\hline 542 & $\mathrm{n}$ & 1.4 & $\mathrm{Cr}$ & $\sigma_{n T}, \quad \sigma_{n X}$ & $3-5$ & $\begin{array}{l}\text { Al Radiator } \\
\text { and Scintilla- } \\
\text { tion Counter } \\
\text { Telescope }\end{array}$ & $\begin{array}{l}\text { Brookhaven, } \\
\text { Cosmotron }\end{array}$ & $\begin{array}{l}\text { ‥ Coor et al., Phys. } \\
\text { Rev. } 98, \frac{1}{2369}(1955)\end{array}$ & $\begin{array}{l}\text { Good and poor } \\
\text { geometry }\end{array}$ \\
\hline 543 & $\mathrm{n}$ & 3.6 & $\mathrm{Cu}$ & $\sigma_{n \times}$ & $\sim 15$ & $\begin{array}{l}\text { Star Density } \\
\text { in Nuclear } \\
\text { Emulsions }\end{array}$ & $\begin{array}{l}\text { University of Cal. } \\
\text { Santa Barbara, } \\
\text { Bevatron }\end{array}$ & $\begin{array}{l}\text { P.H. Barrett, Phys. } \\
\text { Rev. 114, } 1374 \text { (1959) }\end{array}$ & Poor Geometry \\
\hline 544 & $\mathrm{n}$ & $4.5^{\circ}$ & $\mathrm{Cu}$ & $\sigma_{\mathrm{nT}}, \quad \sigma_{\mathrm{nX}}$ & $\sim 5$ & $\begin{array}{l}\text { Counter } \\
\text { Telescope }\end{array}$ & $\begin{array}{l}\text { Berkeley, } \\
\text { Bavatron }\end{array}$ & $\begin{array}{l}\text { J.H. Atkinson et al., } \\
\text { Phys. Rev, Ietters } \\
168 \text { (1959) }\end{array}$ & $\begin{array}{l}\text { Good and poor } \\
\text { geometry }\end{array}$ \\
\hline 545 & $\mathrm{n}$. & 0.090 & $\mathrm{ca}$ & Neutron yield & 10 & $\begin{array}{l}\mathrm{MnSO}_{4} \\
\text { Solution }\end{array}$ & $\begin{array}{l}\text { Berkeley and } \\
\text { Chicago Cyclotrors }\end{array}$ & $\begin{array}{l}\text { W.E. Crandall and G.P. } \\
\text { Miliburn, J. Appl. Phys. } \\
\text { c9 } 698 \text { (1958) }\end{array}$ & \\
\hline 546 & $\mathrm{n}$ & 0.095 & $\mathrm{Sn}$ & $\sigma_{n T}$. & $\approx 5$ & $\begin{array}{l}\text { Bi Fission } \\
\text { Chamber }\end{array}$ & $\begin{array}{l}\text { Berkeley, } \\
\text { Cyclotron }\end{array}$ & $\begin{array}{l}\text { J. DeJuren and N. Knable, } \\
\text { Fhys. Rev. 77, } 606 \text { (1950) }\end{array}$ & $\begin{array}{l}\text { Good and poor } \\
\text { geometry }\end{array}$ \\
\hline 547 & $\mathrm{n}$ & 0.352 & $\mathrm{Sn}$ & $\sigma_{n T}$ & 1 & $\begin{array}{l}\text { Telescope } \\
\text { for Proton } \\
\text { Recoils }\end{array}$ & Liverpool & $\begin{array}{l}\text { A. Ashmore et al., Proc. } \\
\text { Frys. Soc. A(London) } 70 \\
745 \text { (1957) }\end{array}$ & Good geometry \\
\hline 548 & $\mathrm{n}$ & 0.590 & $\mathrm{Sn}$ & $\begin{array}{l}\text { Attempt to show } \\
\pi 0 \text { prcduction } \\
\propto \mathrm{A}^{273}\end{array}$ & - & $\begin{array}{l}\text { Scintillation } \\
\text { Counter Tele } \\
\text { cope and Ceren- } \\
\text { kov Detector }\end{array}$ & $\begin{array}{l}\text { Insititute for } \\
\text { Nuclear Problems, } \\
\text { Synchrocyclotron }\end{array}$ & $\begin{array}{l}\text { V.P. Dzhelepov, K.O. } \\
\text { Oganesian, and V.B. Fliagin, } \\
\text { Soviet Phys.-JETP 5, } 560 \\
\text { (1957) }\end{array}$ & \\
\hline
\end{tabular}




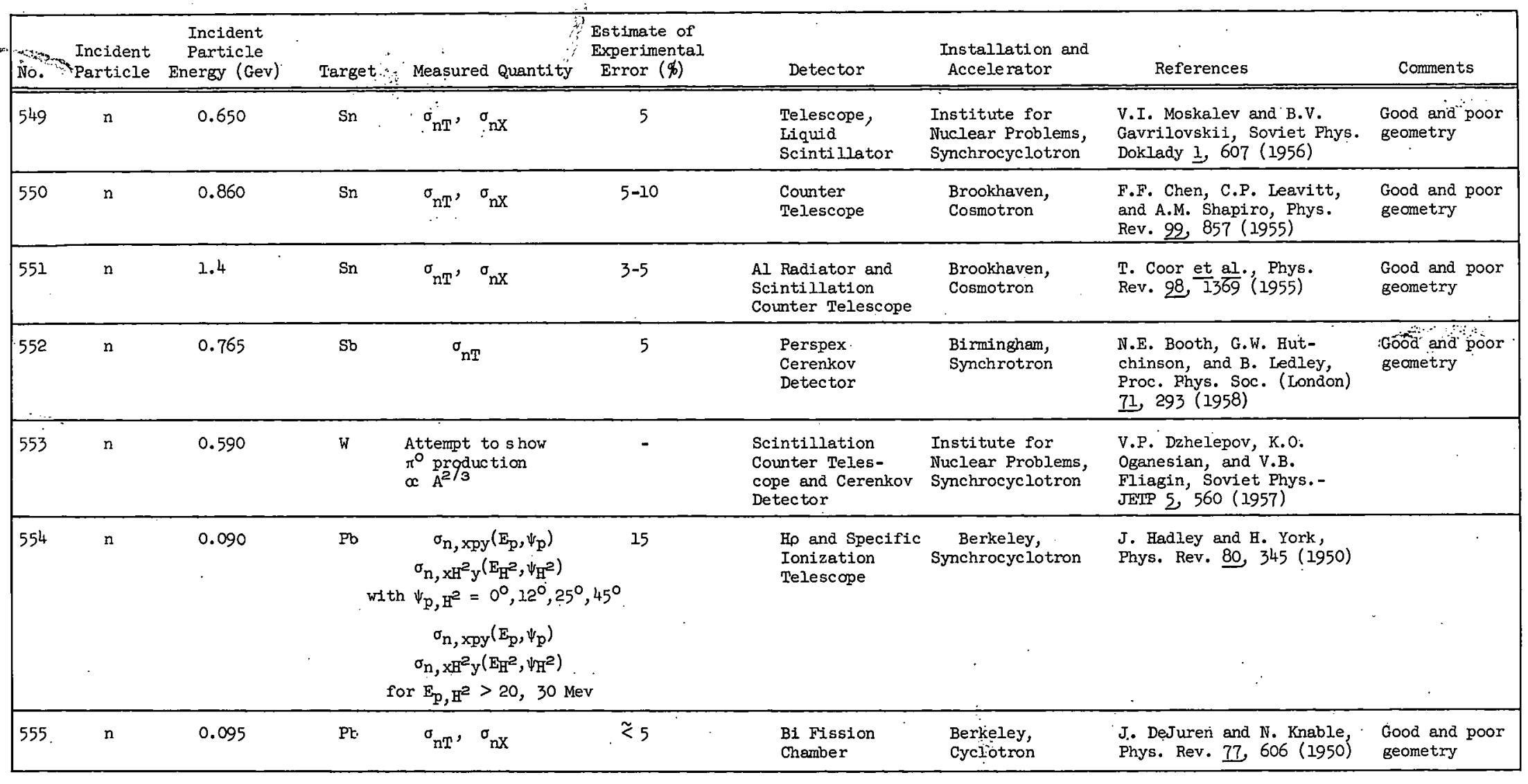




\begin{tabular}{|c|c|c|c|c|c|c|c|c|c|}
\hline No. & $\begin{array}{l}\text { Incident } \\
\text { Particle }\end{array}$ & $\begin{array}{l}\text { Incident } \\
\text { Particle } \\
\text { Energy (Gev) }\end{array}$ & - Target & Measured Quantity & $\begin{array}{l}\text { Estimate of } \\
\text { Experimental } \\
\text { Error }(\%)\end{array}$ & Detector & $\begin{array}{l}\text { Installation and } \\
\text { Accelerator }\end{array}$ & References & Comments \\
\hline 556 & $\mathrm{n}$ & $0.084-0.300$ & $\mathrm{~Pb}$ & $\sigma_{n x}$ & $3-15$ & - & - & $\begin{array}{l}\text { G.P. Millburn et al.' } \\
\text { Pr.ys. Rev. } 95 \text { 1268 (1954) }\end{array}$ & A compilation \\
\hline 557 & $\mathrm{n}$ & 0.352 & $\mathrm{~Pb}$ & $\sigma_{\mathrm{nT}}$ & 1 & $\begin{array}{l}\text { Telescope } \\
\text { for Proton } \\
\text { Recoils }\end{array}$ & Liverpool & $\begin{array}{l}\text { A. Ashmore et al., Proc. } \\
\text { Prys. Soc. A(London) 70, } \\
745 \text { (1957) }\end{array}$ & Good geometry \\
\hline 558 & $\mathrm{n}$ & 0.590 & $\mathrm{~Pb}$ & $\begin{array}{l}\text { Attempt to show } \\
\pi^{\circ} \text { pyoduction } \\
\propto \mathrm{A}^{2 / 3}\end{array}$ & - & $\begin{array}{l}\text { Scintillation } \\
\text { Counter Teles- } \\
\text { cope and Ceren- } \\
\text { kov Detector }\end{array}$ & $\begin{array}{l}\text { Institute for } \\
\text { Nuclear Problems, } \\
\text { Synchrocyclotron }\end{array}$ & $\begin{array}{l}\text { V.P. Dzhelepov, K.O. } \\
\text { Oganesian, and V.B. Fliagin, } \\
\text { Scviet Phys. JETP } \underline{5} \\
56.0 \text { (1957) }\end{array}$ & \\
\hline 559 & $\mathrm{n}$ & 0.650 & $\mathrm{~Pb}$ & $\sigma_{n T}, \quad \sigma_{n X}$ & 5 & $\begin{array}{l}\text { Telescope } \\
\text { Liquid } \\
\text { Scintillator }\end{array}$ & $\begin{array}{l}\text { Institute for } \\
\text { Nuclear Problems, } \\
\text { Synchrocyclotron }\end{array}$ & $\begin{array}{l}\text { V.I. Moskalev and B.v. } \\
\text { Gevrilovskii, Soviet } \\
\text { Plys. Doklady 1, } 607 \text { (1956) }\end{array}$ & $\begin{array}{l}\text { Good and poor } \\
\text { geometry }\end{array}$ \\
\hline 560 & $\mathrm{n}$ & 0.765 & $\mathrm{~Pb}$ & $\sigma_{n T}, \quad \sigma_{n X}$ & 5 & $\begin{array}{l}\text { Perspex } \\
\text { Cerenkov } \\
\text { Detector }\end{array}$ & $\begin{array}{l}\text { Birmingham, } \\
\text { Symchrotron }\end{array}$ & $\begin{array}{l}\text { N.E. Booth, G.W. Hut- } \\
\text { chinson, and B. Ledley, } \\
\text { Proc. Phys. Soc. (London) } \\
\text { 1, } 293 \text { (1958) }\end{array}$ & $\begin{array}{l}\text { Good and poor } \\
\text { geometry }\end{array}$ \\
\hline 561 & $\mathrm{n}$ & 0.860 & $\mathrm{~Pb}$ & $\sigma_{\mathrm{nT}} \quad \sigma_{\mathrm{nX}}$ & $5-10$ & $\begin{array}{l}\text { Counter } \\
\text { Telescope }\end{array}$ & $\begin{array}{l}\text { Brookhaven, } \\
\text { Cosmotron }\end{array}$ & $\begin{array}{l}\text { F.F. Chen, C.P. Leavitt, } \\
\text { and A.M. Shapiro, Phys. } \\
\text { Rev. 29 } 857 \text { (1955) }\end{array}$ & $\begin{array}{l}\text { Good and Poor } \\
\text { geometry }\end{array}$ \\
\hline 562 & n. & - 1.4 & Pb & $\sigma_{n T}, \quad \sigma_{n X}$ & $3-5$ & $\begin{array}{l}\text { Al Radidtor } \\
\text { and Scintilla- } \\
\text { tion Courter } \\
\text { Telescope }\end{array}$ & $\begin{array}{l}\text { Brookhaven, } \\
\text { Cosmotron }\end{array}$ & $\begin{array}{l}\text { T. Coor et al., Phys. } \\
\text { Rev. 98, } 13 \frac{199}{(1955)}\end{array}$ & $\begin{array}{l}\text { Good and poor } \\
\text { geometry }\end{array}$ \\
\hline
\end{tabular}




\begin{tabular}{|c|c|c|c|c|c|c|c|c|c|}
\hline No. & $\begin{array}{l}\text { Iricident } \\
\text { Particle }\end{array}$ & $\begin{array}{c}\text { Incident } \\
\text { Particle } \\
\text { Energy (Gev) }\end{array}$ & Target & Measured Quantity & $\begin{array}{l}\text { Estimate of } \\
\text { Experimental } \\
\text { Error }(\phi)\end{array}$ & Detector & $\begin{array}{l}\text { Installation and } \\
\text { Accelerator }\end{array}$ & References & Comments \\
\hline 563 & $\mathrm{n}$ & 3.6 & Po & $\sigma_{\mathrm{nX}}$ & $\sim 15$ & $\begin{array}{l}\text { Star Density } \\
\text { in Nuclear } \\
\text { Emulsions }\end{array}$ & $\begin{array}{l}\text { University of Cal., } \\
\text { Santa Barbara, } \\
\text { Bevatron }\end{array}$ & $\begin{array}{l}\text { P.H. Barrett, Phys. } \\
\text { Rev. 114, } 1374 \text { (1959) }\end{array}$ & Poor Geometry \\
\hline 564 & $\mathrm{n}$ & 4.5 & $\mathrm{~Pb}$ & $\sigma_{n T}, \sigma_{n x}$ & $\sim 5$ & $\begin{array}{l}\text { Counter } \\
\text { Telescope }\end{array}$ & $\begin{array}{l}\text { Berkeley, } \\
\text { Bevatron }\end{array}$ & $\begin{array}{l}\text { J.H. Atkinson et al. } \\
\text { Phys. Rev. Letters 2, } \\
168 \text { (1959) }\end{array}$ & $\begin{array}{l}\text { Good and poor } \\
\text { geometry }\end{array}$ \\
\hline 565 & $\mathrm{n}$ & 1.4 & $B i$ & $\sigma_{n T}, \sigma_{n X}$ & $3-5$ & $\begin{array}{l}\text { Al Radiator } \\
\text { and Scintilla- } \\
\text { tion Counter } \\
\text { Telescope }\end{array}$ & $\begin{array}{l}\text { Brookhaven, } \\
\text { Cosmotron }\end{array}$ & $\begin{array}{l}\text { T. Coor et al., Phys. } \\
\text { Rev. 98, } 1369 \text { (1955) }\end{array}$ & $\begin{array}{l}\text { Good and poor } \\
\text { geometry }\end{array}$ \\
\hline 566 & $\mathrm{n}$ & 0.090 & Th & Neutron yielá & 10 & $\begin{array}{l}\mathrm{MnSO}_{4} \\
\text { Solution }\end{array}$ & $\begin{array}{l}\text { Berkeley and } \\
\text { Chicago Cyclotrons }\end{array}$ & $\begin{array}{l}\text { W.E. Crandall and G.P. } \\
\text { Millburn, J. Appl. Phys. } \\
\text { 29, } 698 \text { (1958) }\end{array}$ & \\
\hline 567 & $\mathrm{n}$ & 0.090 & $\mathrm{U}$ & Neutron sield & 10 & $\begin{array}{l}\mathrm{MnSO}_{4} \\
\text { Solution }\end{array}$ & $\begin{array}{l}\text { Berkeley and } \\
\text { Chicago Cyclotrons }\end{array}$ & $\begin{array}{l}\text { W.F. Crandall and G.P. } \\
\text { Miliburn, J. Appl. Phys. } \\
\text { 29, } 698 \text { (1958) }\end{array}$ & \\
\hline 568 & $\mathrm{n}$ & 0.095 & U & $\sigma_{n T}$ & $\approx 5$ & $\begin{array}{l}\text { Bi Fission } \\
\text { Chamber }\end{array}$ & $\begin{array}{l}\text { Berkeley, } \\
\text { Cyclotron }\end{array}$ & $\begin{array}{l}\text { J. DeJuren and N. Knable, } \\
\text { Phys. Rev. } 77,606 \text { (1950) }\end{array}$ & $\begin{array}{l}\text { Good and poor } \\
\text { geometry }\end{array}$ \\
\hline 569 & $\mathrm{n}$ & 0.160 & $\mathrm{U}$ & Neutron jield & 20 & $\begin{array}{l}\mathrm{MnSO}_{4} \\
\text { Solution }\end{array}$ & $\begin{array}{l}\text { Berkeley and } \\
\text { Chicago Cyclotrons }\end{array}$ & $\begin{array}{l}\text { W.E. Crandall and G.P. } \\
\text { Millburn, J. Appl. Phys. } \\
\text { 29, } 698 \text { (1958). }\end{array}$ & \\
\hline 570 & $\mathrm{n}$ & 0.650 & $\mathrm{U}$ & $\sigma_{n T}, \sigma_{n X}$ & 5 & $\begin{array}{l}\text { Telescope, } \\
\text { Liquid } \\
\text { Scintillator }\end{array}$ & $\begin{array}{l}\text { Institute for } \\
\text { Nuclear Problems, } \\
\text { Synchrocyclotron }\end{array}$ & $\begin{array}{l}\text { V.I. Moskalev and B.V. } \\
\text { Gavrilovskii, Soviet } \\
\text { Phys. Doklady 1, } 607 \text { (1956) }\end{array}$ & $\begin{array}{l}\text { Good and poor } \\
\text { geometry }\end{array}$ \\
\hline
\end{tabular}




\begin{tabular}{|c|c|c|c|c|c|c|c|c|c|}
\hline No. & $\begin{array}{l}\text { Incident } \\
\text { Particle }\end{array}$ & $\begin{array}{c}\text { Incidert } \\
\text { Particle } \\
\text { Energy (Gev) }\end{array}$ & Target & Measured Quantity & $\begin{array}{l}\text { Estimate of } \\
\text { Experimental } \\
\text { Error }(\%)\end{array}$ & Detector & $\begin{array}{l}\text { Installation and } \\
\text { Accelerator }\end{array}$ & References & Comments \\
\hline 571 & $\mathrm{n}$ & 1.4 & $\mathrm{U}$ & $\sigma_{\mathrm{nT}}, \quad \sigma_{\mathrm{nX}}$ & $3-5$ & $\begin{array}{l}\text { Al Radiator and } \\
\text { Scintillation } \\
\text { Counter Telescope }\end{array}$ & $\begin{array}{l}\text { Brookhaven, } \\
\text { Cosmotron }\end{array}$ & $\begin{array}{l}\text { T. Coor et al., Phys. } \\
\text { Rev. } 98, \frac{1}{1369}(1955)\end{array}$ & $\begin{array}{l}\text { Good and poor } \\
\text { geometry }\end{array}$ \\
\hline 572 & $\mathrm{n}$ & 0.300 & $\begin{array}{l}\text { Heavy Emul- } \\
\text { sion Nuclei }\end{array}$ & $\begin{array}{l}\text { Black, sparse black, } \\
\text { and gray prong num- } \\
\text { ber distribution and } \\
\text { angular distribution }\end{array}$ & 15 & - & $\begin{array}{l}\text { Columbia, } \\
\text { Cyclotron }\end{array}$ & $\begin{array}{l}\text { G. Bernardini, E.T. } \\
\text { Booth, and S.J. } \\
\text { Lindenbaum, Fhys. Rev. } \\
\text { 다 } 826 \text { (1952) }\end{array}$ & \\
\hline 573 & $\mathrm{n}$ & 0.900 & $\begin{array}{l}\text { Heavy Emul- } \\
\text { sion Nuclei }\end{array}$ & $\begin{array}{l}\text { Prong distribution. } \\
\text { Average number of } \\
\text { black, gray, and shower } \\
\text { tracks and angular dis- } \\
\text { tribution of same. }\end{array}$ & $\begin{array}{l}\text { er } \\
\text { s- }\end{array}$ & - & $\begin{array}{l}\text { Birmingham, } \\
\text { Synchrotron }\end{array}$ & $\begin{array}{l}\text { W.T. Morton and B.A. } \\
\text { Munir, Phil. Mag. } \\
\text { 4. } 933 \text { (1959) }\end{array}$ & $\begin{array}{l}\text { Black track E (pro- } \\
\text { ton) }<30 \mathrm{Mev.} \mathrm{Gray} \\
\text { track } 30 \mathrm{Mev} \leq \mathrm{E} \\
\text { (proton) } \leq 450 \mathrm{Mev} \text {. } \\
\text { Shower track } 450 \\
\text { Mev }<\mathrm{E} \text { (proton) }\end{array}$ \\
\hline 574 & $\pi^{+}$ & 0.273 & $\mathrm{He}$ & $\begin{array}{l}\sigma_{\pi^{+}, \pi^{+}}\left(\psi_{\pi^{+}}\right) \\
\sigma_{\pi^{+} x}\end{array}$ & $10-20$ & $\begin{array}{l}\text { Diffusion } \\
\text { (Cloud) } \\
\text { Chamber }\end{array}$ & $\begin{array}{l}\text { Joint Institute } \\
\text { for Nuclear } \\
\text { Research, } \\
\text { Syn=hrocyclotron }\end{array}$ & $\begin{array}{l}\text { M.S. Kozokdaev et al., } \\
\text { Soviet Phys. JETP } \\
300(1960)\end{array}$ & \\
\hline 575 & $\pi^{+}$ & 0.020 & $\mathrm{Be}$ & $\begin{array}{l}\sigma_{\pi^{+}, \pi^{+}}, \sigma_{\pi^{+} D} \text { and } \\
\text { prong distribution }\end{array}$ & 25 & $\begin{array}{l}\text { Cloud } \\
\text { Chamber }\end{array}$ & $\begin{array}{l}\text { Rochester, } \\
\text { Cyclotron }\end{array}$ & $\begin{array}{l}\text { F.H. Tenney and J. } \\
\text { Tinlot, Phys. Rev. } \\
\text { 92, } 974 \text { (1953) }\end{array}$ & \\
\hline 576 & $\pi^{+}$ & 0.030 & $\mathrm{Be}$ & $\begin{array}{l}\sigma_{\pi^{+}, \pi^{+}}, \sigma_{\pi^{+} D} \text { and } \\
\text { prong distribution }\end{array}$ & 25 & Cloud Chamber & $\begin{array}{l}\text { Rochester, } \\
\text { Cyclotron }\end{array}$ & $\begin{array}{l}\text { F.H. Tenney and J. Tinlot, } \\
\text { Phys. Rev. 92, } 974 \text { (1953) }\end{array}$ & \\
\hline 577 & $\pi^{+}$ & 0.040 & $\mathrm{Be}$ & $\begin{array}{l}\sigma_{\pi^{+}, \pi^{+}}, \sigma_{\pi^{+} D} \text { and } \\
\text { prong distribution }\end{array}$ & 25 & $\begin{array}{l}\text { Cloud } \\
\text { Chamber }\end{array}$ & $\begin{array}{l}\text { Rochester, } \\
\text { Cyclotron }\end{array}$ & $\begin{array}{l}\text { F.H. Tenney and J. Tinlot, } \\
\text { Phys. Rev. 92, } 974 \text { (1953) }\end{array}$ & \\
\hline
\end{tabular}




\begin{tabular}{|c|c|c|c|c|c|c|c|c|c|}
\hline No. & $\begin{array}{l}\text { Incident } \\
\text { Particle }\end{array}$ & $\begin{array}{cc} & \text { Incident } \\
\text { t } & \text { Particle } \\
\text { e Energy (Gev) }\end{array}$ & Target & Measured Quantity Es & $\begin{array}{l}\text { Estimate of } \\
\text { Experimental } \\
\text { Error }(\%)\end{array}$ & Detector & $\begin{array}{l}\text { Installation and } \\
\text { Accelerator }\end{array}$ & References & Comments \\
\hline 578 & $\pi^{+}$ & 0.050 & c & $\begin{array}{l}\sigma_{\pi^{+}}+\text {, prong distri- } \\
\text { bution. Angular } \\
\text { distribution of prong } \\
\text { Angular distribution } \\
\text { of 2-prong stars }\end{array}$ & $\begin{array}{l}\text { - } \\
\text { ngs. } \\
\text { n }\end{array}$ & $\begin{array}{l}\text { Propane Bubble } \\
\text { Chamber }\end{array}$ & $\begin{array}{l}\text { Joint Institute } \\
\text { for Nuclear } \\
\text { Research, } \\
\text { Synchrotron }\end{array}$ & $\begin{array}{l}\text { J.V. Laberrigue-Frolova, } \\
\text { M.P. Balandin, and S.z. } \\
\text { Otvinovski1, Soviet Phys. - } \\
\text { JETP 10, } 452 \text { (1960) }\end{array}$ & \\
\hline 579 & $\pi^{+}$ & 0.087 & $\mathrm{C}^{12}$ & $\sigma_{\pi^{+}, \pi^{+}}\left(\psi_{\pi^{+}}{ }^{1}\right)$ & $10-50$ & $\begin{array}{l}\text { Total Aosorp- } \\
\text { tion Scintil- } \\
\text { lation } \\
\text { Spectroneter }\end{array}$ & $\begin{array}{l}\text { Utrecht, Neth.; Ex- } \\
\text { posure Made at CERN } \\
\text { Synchrocyclotron }\end{array}$ & $\begin{array}{l}\text { F.P.G. Valckx, E.S. Gel- } \\
\text { sema, and M. Kruiskamp, } \\
\text { Nuovo cimento 23, } 1005 \\
\text { (1962) }\end{array}$ & \\
\hline 580 & $\pi^{+}$ & 0.060 to 0.125 & $c$ & $\begin{array}{l}\text { Alpha spectra from } \\
\pi+C \rightarrow 3 \alpha . \text { Relative } \\
\sigma_{\pi^{+}, \pi^{+}}{ }^{1}\left(\psi_{\pi^{+}}\right)\end{array}$ & ve & Emulsion & Arcetri, Italy & $\begin{array}{l}\text { M. Della Corte, T.F. } \\
\text { Fazzini, and A.M. Song, } \\
\text { Nuovo cimento 2, } 1345 \\
\text { (1955) }\end{array}$ & . \\
\hline 581 & $\pi^{+}$ & 0.442 & $\mathrm{C}$ & $\sigma_{\pi^{+} X}$ & $\sim 10$ & $\begin{array}{l}\text { Cerenkov } \\
\text { Counters } \\
\text { and Plastic } \\
\text { Scintillators }\end{array}$ & $\begin{array}{c}\text { Berkeley, } \\
\text { Synchrocyclotron }\end{array}$ & $\begin{array}{l}\text { J. Caris et al } \\
\text { Rev. } 126, \frac{\text { Phys. }}{295}(1962)\end{array}$ & $\begin{array}{l}\text { Good and poor } \\
\text { geometry }\end{array}$ \\
\hline 582 & $\pi^{+}$ & $3.7 \mathrm{Gev} / \mathrm{c}$ & c & $\sigma_{\pi^{+} x}$ & $4-20$ & $\begin{array}{l}\text { Ring } \\
\text { Counter }\end{array}$ & $\begin{array}{l}\text { Joint Institute } \\
\text { for Nuclear } \\
\text { Research } \\
\end{array}$ & $\begin{array}{l}\text { M.F. Likhachev et al., } \\
\text { Soviet Phys. JETP } \\
29(1962)\end{array}$ & \\
\hline 583 & $\pi^{+}$ & 0.087 & $0^{18}$ & $\sigma_{\pi^{+}, \pi^{+}}\left(\Psi_{\pi^{+}} !\right)$ & $10-50$ & $\begin{array}{l}\text { Total Absorp- } \\
\text { tion Scintil- } \\
\text { lation } \\
\text { Spectrometer }\end{array}$ & $\begin{array}{l}\text { Utrecht, Neth.; Ex- } \\
\text { posure Made at CERN } \\
\text { Synchrocyclotron }\end{array}$ & $\begin{array}{l}\text { F.P.G. Valckx, E.S. Gel- } \\
\text { sema, and M. Krui skamp, } \\
\text { Nuovo cimento 23, } 1005 \\
\text { (1962) }\end{array}$ & \\
\hline 584 & $\pi^{+}$ & 0.442 & Al & $\sigma_{\pi+x}$ & $\sim 10$ & $\begin{array}{l}\text { Cerenkov Counter } \\
\text { and Plastic } \\
\text { Scintillators }\end{array}$ & $\begin{array}{l}\text { rs Berkeley, } \\
\text { Synchrocyclotron }\end{array}$ & $\begin{array}{l}\text { J. Caris et al.; Phys. } \\
\text { Rev. 126, } \frac{\text { al }}{295}(1962)\end{array}$ & $\begin{array}{l}\text { Good and poor } \\
\text { geometry }\end{array}$ \\
\hline
\end{tabular}




\begin{tabular}{|c|c|c|c|c|c|c|c|c|c|}
\hline No. & $\begin{array}{l}\text { Incident. } \\
\text { Particle }\end{array}$ & $\begin{array}{c}\text { Incident } \\
\text { Particle } \\
\text { Energ (Gev) }\end{array}$ & Target & Measured Quantity & $\begin{array}{l}\text { Estimate of } \\
\text { Experimental } \\
\text { Error }(\$)\end{array}$ & Detector & $\begin{array}{l}\text { Installation and } \\
\text { Accelerator }\end{array}$ & References & Comments \\
\hline 585 & $\pi^{+}$ & $3.7 \mathrm{Gev} / \mathrm{c}$ & Al & $\sigma_{\pi}+\mathrm{X}$ & $4-20$ & Ring Counter & $\begin{array}{l}\text { Joint Institute } \\
\text { for Nuclear } \\
\text { Research }\end{array}$ & $\begin{array}{l}\text { M.F. Likhachev et al., } \\
\text { Soviet Phys. JETP } \\
\text { 29 (1962) }\end{array}$ & . \\
\hline 586 & $\pi^{+}$ & 0.442 & $\mathrm{Cu}$ & $\sigma_{\pi}+x$ & $\sim 10$ & $\begin{array}{l}\text { Cerenkov } \\
\text { Counters and } \\
\text { Plastic } \\
\text { Scintillators }\end{array}$ & $\begin{array}{l}\text { Berkeley, } \\
\text { Synchrocyclotron }\end{array}$ & $\begin{array}{l}\text { J. Caris et al., Phys. } \\
\text { Rev. 126, } \frac{29}{25} \text { (1962) }\end{array}$ & $\begin{array}{l}\text { Good and poor } \\
\text { geometry }\end{array}$ \\
\hline 587 & $\pi^{+}$ & $3.7 \mathrm{G \in V} / \mathrm{c}$ & $\mathrm{Cu}$ & $\sigma_{\pi}+x$ & $4-20$ & Ring Counter & $\begin{array}{l}\text { Joint Institute } \\
\text { for Nuclear } \\
\text { Research }\end{array}$ & $\begin{array}{l}\text { H.F. Likhachev et al., } \\
\text { Soviet Phys. JETP 14, } \\
29(1962)\end{array}$ & . \\
\hline 588 & $\pi^{+}$ & 0.442 & $\mathrm{Cd}$ & $\sigma_{\pi}+x$ & $\sim 10$ & $\begin{array}{l}\text { Cerenkov } \\
\text { Counters and } \\
\text { Plastic } \\
\text { Scintillators }\end{array}$ & $\begin{array}{c}\text { Berkeley, } \\
\text { Synchrocyclotron }\end{array}$ & $\begin{array}{l}\text { J. Caris et al., Phys. } \\
\text { Rev. } 126, \frac{2}{29} \text { (1962) }\end{array}$ & $\begin{array}{l}\text { Good and poor } \\
\text { geometry }\end{array}$ \\
\hline 589. & $\pi^{+}$ & $3.7 \mathrm{Gev} / \mathrm{c}$ & $\mathrm{Sn}$ & $\sigma_{\pi}+x$ & $4-20$ & Ring Counter & $\begin{array}{l}\text { Joint Institute } \\
\text { for Nuclear } \\
\text { Research }\end{array}$ & $\begin{array}{l}\text { M.F. Likhachev et al., } \\
\text { Soviet Phys. JETP } \frac{\text { al }}{14} \text {, } \\
29 \text { (1962) }\end{array}$ & \\
\hline 590 & $\pi^{+}$ & 0.050 & $\mathrm{~Pb}$ & $\begin{array}{l}\sigma_{\pi^{+} X^{\prime}}, \sigma_{\pi^{+}, 1 \pi^{\circ}} \\
\sigma_{\pi^{+}, l p r^{\prime}}, \sigma_{\pi^{+}, 2 p r}\end{array}$ & $10-70$ & Cloud Chamber & $\begin{array}{l}\text { Berkeley, } \\
\text { Synchrocyclotron }\end{array}$ & $\begin{array}{l}\text { G. Saphir, Phys. Rev. } \\
\text { 104, } 535 \text { (1956) }\end{array}$ & \\
\hline 591 & $\pi^{+}$ & 0.5 to 5.0 & $\mathrm{~Pb}$ & $\begin{array}{l}\text { Fraction of primary } \\
\text { energy =emoved by } \pi^{\circ}\end{array}$ & 25 & Cloud Chamber & $\begin{array}{l}\text { Padova, Lab. della. } \\
\text { Marmolada }\end{array}$ & $\begin{array}{l}\text { N. Cresti et al ; Nuovo } \\
\text { cimento } 4,747 \text { (1956) }\end{array}$ & \\
\hline 592 & $\pi^{+}$ & $3.7 \mathrm{Gev} / \mathrm{c}$ & $\mathrm{Pb}$ & $\sigma_{\pi}+\frac{z}{z}$ & $4-20$ & Ring Counter & $\begin{array}{l}\text { Joint Institute } \\
\text { for Nuclear } \\
\text { Research }\end{array}$ & 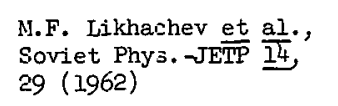 & \\
\hline
\end{tabular}




\begin{tabular}{|c|c|c|c|c|c|c|c|c|c|}
\hline No. & $\begin{array}{l}\text { Incident } \\
\text { Particle }\end{array}$ & $\begin{array}{l}\text { Incident } \\
\text { Particle } \\
\text { Energy (Gev) }\end{array}$ & Target & $\begin{array}{r}\text { Esti } \\
\text { Expe } \\
\text { Measured Quantity }\end{array}$ & $\begin{array}{l}\text { imate of } \\
\text { erimental } \\
\text { ror }(\phi)\end{array}$ & Detector & $\begin{array}{l}\text { Installation and } \\
\text { Accelerator }\end{array}$ & References & Comments \\
\hline 593 & $\pi^{+}$ & 0.280 & $\begin{array}{l}\text { Light Emul- } \\
\text { sion Nuclei }\end{array}$ & $\begin{array}{l}\text { Charge distribution } \\
\text { of fragments. Energy } \\
\text { distribution of frag- } \\
\text { ments. Angular dis- } \\
\text { tribution of fragments }\end{array}$ & $10-30$ & - & $\begin{array}{l}\text { Radium Institute; Ex- } \\
\text { posure Made at Joint } \\
\text { Institute for Nuclear. } \\
\text { Research } \\
\text { Synchrocyclotron }\end{array}$ & $\begin{array}{l}\text { N.S. Ivanova, V.I. } \\
\text { Ostroumov, and Yu.V. } \\
\text { Pavlov, Soviet Phys.- } \\
\text { JEIP 10, } 1137 \text { (1960) }\end{array}$ & . \\
\hline .594 & $\pi^{+}$ & 0.280 & $\begin{array}{l}\text { Heavy Emul- } \\
\text { sion Nuclei }\end{array}$ & $\begin{array}{l}\text { Cherge distribution } \\
\text { of fragments. Energy } \\
\text { distribution of frag- } \\
\text { ments. Angular dis- } \\
\text { tribution of fragments }\end{array}$ & $10-30$ & - & $\begin{array}{l}\text { Radium Institute; Ex- } \\
\text { posure Made at Joint } \\
\text { Institute for Nuclear } \\
\text { Research } \\
\text { Synchrocyclotron }\end{array}$ & $\begin{array}{l}\text { N.S. Ivanova, V.I. } \\
\text { Ostroumov, and Yu.v. } \\
\text { Pavlov, Soviet Phys.- } \\
\text { JEIP 10, } 1137 \text { (1960) }\end{array}$ & $\therefore$ \\
\hline 595 & $\pi^{+}$ & 0.080 & $\begin{array}{l}\text { Emulssion } \\
\text { Nucles. }\end{array}$ & $\begin{array}{l}\sigma_{\pi^{+}}{ }^{+} \text {fr, charge distri- } \\
\text { bution, energy distri- } \\
\text { bution, and angular } \\
\text { distribution of frag- } \\
\text { ments ( } \mathrm{z} \geq 4 \text { ) from } \\
\text { heavy nuclei }\end{array}$ & 50 & - & $\begin{array}{l}\text { Radium Institute; } \\
\text { Plates Exposed at } \\
\text { Joint Institute } \\
\text { for Nuclear } \\
\text { Research } \\
\text { Synchrocyclotron }\end{array}$ & $\begin{array}{l}\text { A.S. Assovskaya and N.s. } \\
\text { Ivanova, Soviet Phys. - } \\
\text { JETP 12, 1051 (1961) } \\
\end{array}$ & . \\
\hline 596 & $\pi^{+}$ & 0.078 & Propane & $\begin{array}{l}\sigma_{\pi^{+}} \text {on C. Prongs } \\
\text { emitted forward } \\
\text { and backward for } \\
\pi^{+} \text {absorption }\end{array}$ & $20-40$ & $\begin{array}{l}\text { Bubble } \\
\text { Chamber }\end{array}$ & $\begin{array}{l}\text { Joint Institute } \\
\text { for Nuclear } \\
\text { Research, } \\
\text { Symchrocyclotron }\end{array}$ & $\begin{array}{l}\text { R.G. Sulukvadze and D. } \\
\text { Neagu, Soviet Phys.- } \\
\text { JETP 14, } 59 \text { (1962) }\end{array}$ & \\
\hline 597 & $\pi^{+} 0.080$ & 10 to 0.300 & $\begin{array}{r}\text { Mixture } \\
\text { of } C, F, C 1\end{array}$ & $\sigma_{\pi}+x$ & 10 & $\begin{array}{l}\text { Freon Bubble } \\
\text { Chamber }\end{array}$ & $\begin{array}{l}\text { Joint Insti tute } \\
\text { for Nuclear } \\
\text { Research, } \\
\text { Synchrocyclotron }\end{array}$ & $\begin{array}{l}\text { A.G. Meshkovskii and } \\
\text { Ia. Ia. Shalamov, Soviet } \\
\text { Phys. JETP 10. } 697 \text { (1960) }\end{array}$ & \\
\hline 598 & $\pi^{-}$ & 0.330 & . $\mathrm{He}$ & $\begin{array}{c}\sigma_{\pi^{-}, \pi^{-1}}\left(\psi_{\pi^{-1}}\right) \\
\sigma_{\pi-x}\end{array}$ & $10-20$ & $\begin{array}{l}\text { Diffusion } \\
\text { (Cloud) } \\
\text { Chamber }\end{array}$ & $\begin{array}{l}\text { Joint Institute } \\
\text { for Nuclear } \\
\text { Research, } \\
\text { Synchrocyclotron }\end{array}$ & $\begin{array}{l}\text { M.S. Kozodaev et al., } \\
\text { Soviet Phys. JWTP } 100 \text { (1960) } \\
300 \text {, }\end{array}$ & . \\
\hline
\end{tabular}




\begin{tabular}{|c|c|c|c|c|c|c|c|c|c|c|}
\hline No. & $\begin{array}{l}\text { Incident } \\
\text { Particle }\end{array}$ & $\begin{array}{cc}\text { Incident } \\
\text { Particle } \\
\text { energy (Gev) }\end{array}$ & Target & Measured & Quantity $\frac{E}{E}$ & $\begin{array}{l}\text { Estimate of } \\
\text { Experimental } \\
\text { Error }(\%)\end{array}$ & Detector & $\begin{array}{l}\text { Installation and } \\
\text { Accelerator }\end{array}$ & References & Comments \\
\hline 599 & $\pi^{-}$ & 0.140 & $\mathrm{Be}$ & $\sigma_{\pi-T^{\prime}}$ & $\sigma_{\pi-x}$ & $5-10$ & - & - & $\begin{array}{l}\text { A.E. Ignatenko et al., } \\
\text { Soviet Phys. JETP } \frac{\frac{1}{4}}{35 \text { (1957) }}\end{array}$ & \\
\hline 600 & $\pi^{-}$ & 0.216 & $\mathrm{Be}$ & $\sigma_{\pi-T}$ & $\sigma_{\pi-x}$ & $5-10$ & - & - & $\begin{array}{l}\text { A.E. Ignatenko et al., } \\
\text { Soviet Phys. JETP } \frac{\text { al }}{4} \\
35 \text { (1957). }\end{array}$ & \\
\hline 601 & $\pi^{-}$ & 0.256 & $\mathrm{Be}$ & $\sigma_{\pi-T}$ & $\sigma_{\pi-x}$ & $5-10$ & - & - & $\begin{array}{l}\text { A.E. Ignatenko et al., } \\
\text { Soviet Phys. JETP } \frac{4}{4} \\
35 \text { (1957) }\end{array}$ & \\
\hline 602 & $\pi^{-}$ & 0.290 & $\mathrm{Be}$ & $\sigma_{\pi-T}{ }^{\prime}$ & $\sigma_{\pi \mathrm{x}}$ & $5-10$ & - & - & $\begin{array}{l}\text { A.E. Ignatenko et al., } \\
\text { Soviet Phys. JETP } \frac{1}{4} \\
\text { 35 (1957) }\end{array}$ & \\
\hline 603 & $\pi^{-}$ & 0.350 & $\mathrm{Be}$ & $\sigma_{\pi-T^{\prime}}$ & $\sigma_{\pi-x}$ & $5-10$ & - & - & $\begin{array}{l}\text { A.E. Ignatenko et al., } \\
\text { Soviet Phys. JETP } \frac{14}{4} \text { (1957) }\end{array}$ & \\
\hline 604 & $\pi^{-}$ & $0.960 \mathrm{Gev} / \mathrm{c}$ & $\mathrm{Be}$ & $\begin{array}{l}\sigma_{\pi^{-},}, y \\
\text { with } 0^{\circ}\end{array}$ & $\begin{array}{l}\pi^{-} y^{(}\left(\psi_{\pi^{-}}\right) \\
\leq \psi_{\pi^{-}} \leq 16^{\circ}\end{array}$ & · & $\begin{array}{l}\text { Plastic } \\
\text { Scintillator } \\
\text { Telescope }\end{array}$ & $\begin{array}{l}\text { College of France, } \\
\text { Proton Synchrotron }\end{array}$ & $\begin{array}{l}\text { N. Crozon and J. Tocque- } \\
\text { ville, Compt. rend. } 254 \\
1963 \text { (1962) }\end{array}$ & \\
\hline 605 & $\pi^{-}$ & 0.970 & $\mathrm{Be}$ & $\sigma_{\pi \tau}$ & & 5 & $\begin{array}{l}\text { Toluene- } \\
\text { Terphenyl } \\
\text { Scintilletor }\end{array}$ & $\begin{array}{l}\text { Brookhaven, } \\
\text { Cosmotron }\end{array}$ & $\begin{array}{l}\text { J.W. Cronin, R. Cool, and } \\
\text { P. Abashian, Phys. Rev. } \\
\text { 107, } 1121 \text { (1957) }\end{array}$ & \\
\hline 606 & $\pi^{-} \quad 0$ & 0.360 to 0.125 & $\mathrm{C}$ & $\begin{array}{l}\text { Alpha sp } \\
\text { from } \pi+ \\
\text { Angular } \\
\text { of inela } \\
\text { tering }\end{array}$ & $\begin{array}{l}\text { ectrum } \\
c \rightarrow 3 \alpha \text {. } \\
\text { distribution } \\
\text { ustic scat- }\end{array}$ & on & Enoulsion & Arcetri, Italy & $\begin{array}{l}\text { M. Della Corte, T.F. } \\
\text { Pazzini, and A.M. Song, } \\
\text { Nuovo cimento 2, } 1345 \\
\text { (1955) }\end{array}$ & \\
\hline
\end{tabular}




\begin{tabular}{|c|c|c|c|c|c|c|c|c|c|}
\hline No. & $\begin{array}{l}\text { Incident } \\
\text { Particle }\end{array}$ & $\begin{array}{cc} & \text { Incident } \\
\text { t } & \text { Particle } \\
\text { e Energy (Gev) }\end{array}$ & Target & $\begin{array}{c}\text { Es } \\
\text { Ex } \\
\text { Measured Quantity } \\
\text { E }\end{array}$ & $\begin{array}{l}\text { Estimate of } \\
\text { Experimental } \\
\text { Error }(\%)\end{array}$ & Detector & $\begin{array}{l}\text { Installation and } \\
\text { Accelerator }\end{array}$ & References & Comments \\
\hline 607 & $\pi^{-}$ & 0.125 & $\mathrm{c}$ & $\begin{array}{l}\sigma_{\pi-, \pi^{-1}}\left(E_{\pi^{-1}}, \psi_{\pi^{-1}}\right) \\
\text { for } E_{\pi^{-1}}<85 \mathrm{Mev} ; \\
\sigma_{\pi^{-} \eta^{j}} \sigma_{\pi^{-}, 1 \pi^{\circ} ;} \\
\sigma_{\pi^{-}}+\sigma_{\pi^{-}, 1 \pi^{\circ}}\end{array}$ & 20 & $\begin{array}{l}\text { Cloud } \\
\text { Chamber }\end{array}$ & $\begin{array}{l}\text { Columbia, } \\
\text { Cyclotron }\end{array}$ & $\begin{array}{l}\text { J.0. Kessler and L.M. } \\
\text { Lederman, Phys. Rev. } \\
\text { 94, } 689(1954)\end{array}$ & . \\
\hline 608 & $\pi^{-}$ & 0.230 to 0.250 & $\mathrm{c}$ & $\begin{array}{c}\sigma_{\pi-x} \\
\sigma_{\pi^{-}, \pi^{-1}\left(\psi_{\pi^{-}}-1\right)}\end{array}$ & 10 & $\begin{array}{l}\text { Cloud } \\
\text { Chamber }\end{array}$ & $\begin{array}{l}\text { Inștitute for } \\
\text { Nuclear Problems, } \\
\text { Synchrocyclotron }\end{array}$ & $\begin{array}{l}\text { V.P. Dahelepov et al., } \\
\text { Soviet Phys. JETP } \frac{a}{4} \\
864 \text { (1957) }\end{array}$ & \\
\hline 609 & $\pi^{-}$ & 0.6 & c & $\sigma_{\pi-x}$ & 5 & $\begin{array}{l}\text { Toluene- } \\
\text { Terphenyl } \\
\text { Scintillator }\end{array}$ & $\begin{array}{l}\text { Brookheven, } \\
\text { Cosmotron }\end{array}$ & $\begin{array}{l}\text { J.W. Cronin, R. Cool, } \\
\text { and A. Abashian, Phys. } \\
\text { Rev. 107, } 1121 \text { (1957) }\end{array}$ & \\
\hline 610 & $\pi^{-}$ & 0.8 & C & $\sigma_{\pi x}$ & 5 & $\begin{array}{l}\text { Toluene- } \\
\text { Terphenyl } \\
\text { Scintillator }\end{array}$ & $\begin{array}{l}\text { Brookhaven, } \\
\text { Cosmotron }\end{array}$ & $\begin{array}{l}\text { J.w. Cronin, R. Cool, } \\
\text { and A. Abashian, Phys. } \\
\text { Rev. 107, } 1121 \text { (1957) }\end{array}$ & . \\
\hline 611 & $\pi^{-}$ & 0.915 & $\mathrm{C}$ & $\begin{array}{l}\sigma_{\pi-T} ;-\sigma_{\pi}-\pi-1 ; \text { prong } \\
\text { distribution, } \pi^{-} \\
\text {momentum spectrum } \\
\text { and angular distribu- } \\
\text { tion for events with } \\
\text { various prongs }\end{array}$ & 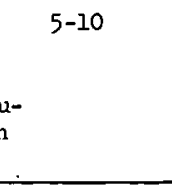 & $\begin{array}{l}\text { Propane } \\
\text { Bubble } \\
\text { Chamber }\end{array}$ & $\begin{array}{l}\text { Bari, Italy; } \\
\text { Exposure Made at } \\
\text { Brookhaven } \\
\text { Cosmotron }\end{array}$ & $\begin{array}{l}\text { N. Abbattista et al., } \\
\text { Nuovo cimento } \frac{\text { al }}{23}, \frac{1}{1} \\
\text { (1962) }\end{array}$ & \\
\hline 612 & $\pi^{-}$ & $0.960 \mathrm{Gev} / \mathrm{c}$ & $\mathrm{c}$ & $\begin{array}{l}\quad \sigma_{\pi^{-}}, x \pi^{-} y^{-}\left(\psi_{\pi^{-}}\right) \\
\text {with } 0^{\circ} \leq \psi_{\pi^{-}} \leq 16^{\circ}\end{array}$ & 10 & $\begin{array}{l}\text { Plastic Scin- } \\
\text { tillator } \\
\text { Telescope }\end{array}$ & $\begin{array}{l}\text { College of France, } \\
\text { Proton Synchrotron }\end{array}$ & $\begin{array}{l}\text { M. Crozon and J. Tocque- } \\
\text { ville, Compt. rend. } 254 \\
1963 \text { (1962) }\end{array}$ & \\
\hline 613 & $\pi^{-}$ & 0.97 & C & $\sigma_{\pi-x}$ & 5 & $\begin{array}{l}\text { Toluene- } \\
\text { Terphenyl } \\
\text { Scintillator }\end{array}$ & $\begin{array}{l}\text { Brookhaven, } \\
\text { Cosmotron }\end{array}$ & $\begin{array}{l}\text { J.W. Cronin, R. Cool, } \\
\text { and A. Abashian, Phys. } \\
\text { Rev. 107, } 1121 \text { (1957) }\end{array}$ & \\
\hline 614 & $\pi^{-}$ & 1.20 & C & $\sigma_{\pi-X}$ & 5 & $\begin{array}{l}\text { Toluene- } \\
\text { Terphenyl } \\
\text { Scintillator }\end{array}$ & $\begin{array}{l}\text { Brookhaven, } \\
\text { Cosmotron }\end{array}$ & $\begin{array}{l}\text { J.W. Cronin, R. Cool, } \\
\text { and A. Abashian, Phys. } \\
\text { Rev. 107, } 1121 \text { (1957) }\end{array}$ & . \\
\hline
\end{tabular}




\begin{tabular}{|c|c|c|c|c|c|c|c|c|c|}
\hline No. & $\begin{array}{l}\text { Incident } \\
\text { Particle }\end{array}$ & $\begin{array}{l}\text { Incident } \\
\text { Particle } \\
\text { Energy (Gev) }\end{array}$ & Target & Measured Quantity & $\begin{array}{l}\text { Estimate of } \\
\text { Experimental } \\
\text { Error }(\%)\end{array}$ & Detector & $\begin{array}{l}\text { Installation and } \\
\text { Accelerator }\end{array}$ & References & Comments \\
\hline 615 & $\pi^{-}$ & 1.5 & c & $\sigma_{\pi \mathrm{X}}$ & 5 & $\begin{array}{l}\text { Cloud } \\
\text { Chamber }\end{array}$ & $\begin{array}{l}\text { Brookhaven, } \\
\text { Cosmotron }\end{array}$ & $\begin{array}{l}\text { T. Bowen et al., Nuovo } \\
\text { cimento } 2,908 \text { (1958) }\end{array}$ & \\
\hline 616 & $\pi^{-}$ & 1.2 & I & $\begin{array}{l}\text { Momenta, angular } \\
\text { and charge dist. } \\
\text { of various fragments }\end{array}$ & s & $\begin{array}{l}\text { Cloud } \\
\text { Chamber }\end{array}$ & $\begin{array}{l}\text { Brookhaven, } \\
\text { Cosmotron }\end{array}$ & $\begin{array}{l}\text { G.D. Gordon et al., } \\
\text { P.nys. Rev. } 108, \frac{1}{1} 315 \\
\text { (1957) }\end{array}$ & \\
\hline 617 & $\pi^{-}$ & 0.140 & 0 & $\sigma_{\pi-T}, \sigma_{\pi-X}$ & $5-10$ & - & - & $\begin{array}{l}\text { A.E. Ignatenko et } \frac{a l}{4} \cdot \text {, } \\
\text { Soviet Phys. JETP } \\
\text { (1957) }\end{array}$ & \\
\hline 618 & $\pi^{-}$ & 0.216 & 0 & $\sigma_{\pi-T}, \sigma_{\pi X X}$ & $5-10$ & - & - & $\begin{array}{l}\text { A.E. Ignatenko et al., } \\
\text { Soviet Phys. JETP } \frac{1}{4} 35 \\
\text { (1957) }\end{array}$ & \\
\hline 619 & $\pi^{-}$ & 0.256 & 0 & $\sigma_{\pi-T}, \sigma_{\pi x}$ & $5-10$ & - & - & $\begin{array}{l}\text { A.I. Ignatenko et al., } \\
\text { Soviet Phys. JETP } \frac{1}{4} 35 \\
\text { (1957) }\end{array}$ & \\
\hline $\begin{array}{c}620 \\
\therefore\end{array}$ & $\pi^{-}$ & $\because \quad \begin{array}{l}0.290 \\
\because \quad\end{array}$ & 0 & $\sigma_{\pi-T}, \sigma_{\pi-X}$ & $5-10$ & - & - & $\begin{array}{l}\text { A.E. Ignatenko et al., } \\
\text { Soviet Phys. JETP } \frac{\text { al }}{4} 35 \\
\text { (1957) }\end{array}$ & . \\
\hline 621 & $\pi^{-}$ & 0.350 & D & $\sigma_{\pi^{-T}}, \sigma_{\pi^{-} X}$ & $5-10$ & - & - & $\begin{array}{l}\text { A.E. Ignatenko et al., } \\
\text { Soviet Phys. -JETP } \frac{14}{4} 35 \\
\text { (1957) }\end{array}$ & \\
\hline 622 & $\pi^{-}$ & 0.970 & Al & $\sigma_{\pi-\mathrm{X}}$ & 5 & $\begin{array}{l}\text { Toluene- } \\
\text { Terphenyl } \\
\text { Scintillator }\end{array}$ & $\begin{array}{l}\text { Brookhaven, } \\
\text { Cosmotron }\end{array}$ & $\begin{array}{l}\text { J.W. Cronin, R. Cool, and } \\
\text { A. Abashian, Phys. Rev. } \\
107 \text {, } 1121 \text { (1957) }\end{array}$ & . \\
\hline 623 & $\pi^{-}$ & 0.970 & $\mathrm{Ca}$ & $\sigma_{\pi-\mathrm{X}}$ & 5 & $\begin{array}{l}\text { Toluene- } \\
\text { Terphenyl } \\
\text { Scintillator }\end{array}$ & $\begin{array}{l}\text { Brookhaven, } \\
\text { Cosmotron }\end{array}$ & $\begin{array}{l}\text { J.W. Cronin, R. Cool, and } \\
\text { A. Abashian, Phys. Rev. } \\
\text { 107, } 1121 \text { (1957) }\end{array}$ & \\
\hline
\end{tabular}




\begin{tabular}{|c|c|c|c|c|c|c|c|c|c|}
\hline No. & $\begin{array}{l}\text { Incident } \\
\text { Particle }\end{array}$ & $\begin{array}{c}\text { Incident } \\
\text { Particle } \\
\text { Energy (Gev) }\end{array}$ & Target & Measured Quantity & $\begin{array}{l}\text { Estimate of } \\
\text { Experimental } \\
\text { Error }(\phi)\end{array}$ & Detector & $\begin{array}{l}\text { Installation and } \\
\text { Accelerator }\end{array}$ & References & Conments \\
\hline 624 & $\pi^{-}$ & 1.5 & $\mathrm{Fe}$ & $\sigma_{\pi x}$ & 5 & $\begin{array}{l}\text { Cloud } \\
\text { Chamber }\end{array}$ & $\begin{array}{l}\text { Brookhaven, } \\
\text { Cosmotron }\end{array}$ & $\begin{array}{l}\text { T. Bowen et al., Nuovo } \\
\text { cimento 9, } 908 \text { (1958) }\end{array}$ & \\
\hline 625 & $\pi^{-}$ & 0.140 & $\mathrm{Cu}$ & $\sigma_{\pi-T}, \sigma_{\pi-x}$ & $5-10$ & - & - & $\begin{array}{l}\text { A.E. Ignatenko et al., } \\
\text { Soviet Phys. -JETP } \\
35 \text { (1957) }\end{array}$ & ' \\
\hline 626 & $\pi^{-}$ & 0.216 & $\mathrm{Cu}$ & $\sigma_{\pi}-T, \sigma_{\pi X}$ & $5-10$ & - & - & $\begin{array}{l}\text { A.E. Ignatenko et al } \\
\text { Soviet Phys. -JETT } \frac{\text { al }}{4} \\
35 \text { (1957) }\end{array}$ & $\cdot$ \\
\hline 627 & $\pi^{-}$ & 0.256 & $\mathrm{Cu}$ & $\sigma_{\pi-T}, \sigma_{\pi-X}$ & $5-10$ & - & - & $\begin{array}{l}\text { A.E. Ignatenko et al. } \\
\text { Soviet Phys. JEEP } \frac{\text { Let }}{4} \\
35 \text { (1957) }\end{array}$ & \\
\hline 628 & $\pi^{-}$ & 0.290 & $\mathrm{Cu}$ & $\sigma_{\pi-T}, \sigma_{\pi x}$ & $5-10$ & - & - & $\begin{array}{l}\text { A.E. Ignatenko et al., } \\
\text { Soviet Phys. -JETP } \frac{1}{4} \\
35 \text { (1957) }\end{array}$ & \\
\hline 629 & $\pi^{-}$ & 0.350 & $\mathrm{Cu}$ & $\sigma_{\pi-T}, \sigma_{\pi-X}$ & $5-10$ & - & - & $\begin{array}{l}\text { A.E. Ignatenko et al., } \\
\text { Soviet Phys. JETP } \frac{\text { I }}{4} \text {. } \\
35 \text { (1957) }\end{array}$ & \\
\hline 630 & $\pi^{-}$ & $0.960 \mathrm{Gev} / \mathrm{c}$ & $\mathrm{Cu}$ & $\begin{array}{c}\sigma_{\pi^{-}, x \pi}=\left(\psi\left(\psi_{\pi^{-}}\right)\right. \\
\text {with } 0^{\circ} \leq \psi_{\pi^{-}} \leq 16^{\circ}\end{array}$ & 10 & $\begin{array}{l}\text { Plastic } \\
\text { Scintillator. } \\
\text { Telescope }\end{array}$ & $\begin{array}{l}\text { College of France, } \\
\text { Proton Synchrotron }\end{array}$ & $\begin{array}{l}\text { M. Crozon and J. Tocque- } \\
\text { ville, Compt. rend. } 254 \text {, } \\
1963 \text { (1962) }\end{array}$ & \\
\hline 631 & $\pi^{-}$ & 0.970 & $\mathrm{Cu}$ & $\sigma_{n}-x$ & 5 & $\begin{array}{l}\text { Toluene- } \\
\text { Terphenyl } \\
\text { Sẹintillator }\end{array}$ & $\begin{array}{l}\text { Brookhaven, } \\
\text { Cosmotron }\end{array}$ & $\begin{array}{l}\text { J.W. Cronin, R. Cool, and } \\
\text { A. Abashian, Phys. Rev. } \\
\text { 107 } 1121 \text { (I957) }\end{array}$ & \\
\hline 632 & $\pi^{-}$ & $6.0 \mathrm{Gev} / \mathrm{c}$ & $\mathrm{Cu}$ & $\sigma_{\pi-x}$ & 3 & $\begin{array}{l}\text { Freon Bubble } \\
\text { Chamber }\end{array}$ & $\begin{array}{l}\text { Un. of Fribourg, } \\
\text { Switz.; Exposure } \\
\text { Made at CERN } \\
\text { Synchrotron }\end{array}$ & $\begin{array}{l}\text { R.N. Peacock et al., } \\
\text { Nuovo cimento } 22 \text {, } 1290 \\
\text { (1961) }\end{array}$ & \\
\hline
\end{tabular}




\begin{tabular}{|c|c|c|c|c|c|c|c|c|}
\hline No. & $\begin{array}{l}\text { Incident } \\
\text { Particle }\end{array}$ & $\begin{array}{c}\text { Incident } \\
\text { Particle } \\
\text { Energy (Gev) }\end{array}$ & Target & Measured Quantity & $\begin{array}{l}\text { Estimate of } \\
\text { Experimental } \\
\text { Error }(\%)\end{array}$ & Detector & $\begin{array}{l}\text { Installation and } \\
\text { Accelerator }\end{array}$ & References \\
\hline 633 & $\pi^{-}$ & $11.3 \mathrm{Gev} / \mathrm{c}$ & $\mathrm{Ou}$ & $\sigma_{\pi}-X$ & 3 & $\begin{array}{l}\text { Freon } \\
\text { Bubble } \\
\text { Chamber }\end{array}$ & $\begin{array}{l}\text { Un. of Fribourg, } \\
\text { Switz.j CERN } \\
\text { Synchrotron }\end{array}$ & $\begin{array}{l}\text { F.N. Peacock et al. } \\
\text { Nuovo cimento } 22,1290 \\
\text { (1961) }\end{array}$ \\
\hline 634 & $\pi^{-}$ & $16.0 \mathrm{Gev} / \mathrm{c}$ & $\mathrm{Iu}$ & $\sigma_{\pi}-x$ & 3 & $\begin{array}{l}\text { Freon } \\
\text { Bubble } \\
\text { Chamber }\end{array}$ & $\begin{array}{l}\text { Un. of Fribourg, } \\
\text { Switz.; CERN } \\
\text { Synchrotron }\end{array}$ & $\begin{array}{l}\text { F.N. Peacock et al., } \\
\text { nuovo cimento } 22,1290 \\
\text { (1961) }\end{array}$ \\
\hline 635 & $\pi^{-}$ & $17.6 \mathrm{Gev} / \mathrm{c}$ & $\approx u$ & $\sigma_{\pi}-x$ & 3 & $\begin{array}{l}\text { Freon } \\
\text { Bubble } \\
\text { Chamber }\end{array}$ & $\begin{array}{l}\text { Un. of Fribourg, } \\
\text { Switz.; CERN } \\
\text { Synchrotron }\end{array}$ & $\begin{array}{l}\text { R.N. Peacock et al. } \\
\text { Nuovo cimento 22, } 1290 \\
\text { (1961) }\end{array}$ \\
\hline 636 & $\pi^{-}$ & $\begin{array}{c}0.122 \\
\text { initially }\end{array}$ & $\stackrel{\mathrm{Ag}}{\text { (thick) }}$ & $\begin{array}{l}\text { Radio-chemical } \\
\text { cross sections }\end{array}$ & $\sim 15$ & $\begin{array}{l}\text { Radio-Chemical } \\
\text { Techniques }\end{array}$ & $\begin{array}{l}\text { Chicago, } \\
\text { Synchrocyclotron }\end{array}$ & $\begin{array}{l}\text { W. Goishi and W.F. Tibby, } \\
\text { Phys. Rev. 104, } 1717 \text { (1956) }\end{array}$ \\
\hline 637 & $\pi^{-}$ & $6.0 \mathrm{Gev} / \mathrm{c}$ & $\mathrm{Cd}$ & $\sigma_{\pi x}$ & 3 & $\begin{array}{l}\text { Freoon } \\
\text { Bubble } \\
\text { Chamber }\end{array}$ & $\begin{array}{l}\text { Un. of Fribourg, } \\
\text { Switz; CERN } \\
\text { Synchrotron }\end{array}$ & $\begin{array}{l}\text { R.N. Peacoek et al. } \\
\text { Nuovo cimento } 22,1290 \\
\text { (1961) }\end{array}$ \\
\hline 638 & $\pi^{-}$ & $11.3 \mathrm{Gev} / \mathrm{c}$ & $\mathrm{Cd}$ & $\sigma_{\pi}-\mathrm{x}$ & 3 & $\begin{array}{l}\text { Freon } \\
\text { Bubble } \\
\text { Chamber }\end{array}$ & $\begin{array}{l}\text { Un. of Fribourg, } \\
\text { Switz.; CERN } \\
\text { Synchrotron }\end{array}$ & $\begin{array}{l}\text { R.N. Peacock et al., } \\
\text { Nuovo cimento } 22 \text {, } 1290 \\
\text { (1961) }\end{array}$ \\
\hline 639 & $\pi^{-}$ & $16.0 \mathrm{Gev} / \mathrm{c}$ & $\mathrm{Cd}$ & $\sigma_{\pi}-x$ & 3 & $\begin{array}{l}\text { Freon } \\
\text { Bubble } \\
\text { Chamber }\end{array}$ & $\begin{array}{l}\text { Un. of Fribourg, } \\
\text { Switz.; CERN } \\
\text { Synchrotron }\end{array}$ & $\begin{array}{l}\text { R.N. Peacosk et al:' } \\
\text { Nuovo cimento } 22 \text { íl } \\
\text { (1961) }\end{array}$ \\
\hline 640 & $\pi^{-}$ & $17.6 \mathrm{Gev} / \mathrm{c}$ & $\mathrm{Cd}$ & $\sigma_{\pi}-X$ & 3 & $\begin{array}{l}\text { Freon } \\
\text { Bubble } \\
\text { Chamber }\end{array}$ & $\begin{array}{l}\text { Un. of Fribourg, } \\
\text { Switz.; CERN } \\
\text { Synchrotron }\end{array}$ & $\begin{array}{l}\text { R.N. Peacos } \mathrm{k} \text { et al., } \\
\text { Muovo cimento } 22,1290 \\
\text { (1961) }\end{array}$ \\
\hline 641 & $\pi^{-}$ & 0.970 & $\mathrm{Sn}$ & $\sigma_{\pi x}$ & 5 & $\begin{array}{l}\text { Toluene- } \\
\text { Terphenjl } \\
\text { Scintillator }\end{array}$ & $\begin{array}{l}\text { Brookhaven, } \\
\text { Cosmotron }\end{array}$ & $\begin{array}{l}\text { ¿.W. Cronin, R. Cool, and } \\
\text { A. Abashian, Phys. Rev. } 107 \text {, } \\
\text { li2l (1957) }\end{array}$ \\
\hline
\end{tabular}




\begin{tabular}{|c|c|c|c|c|c|c|c|c|c|}
\hline No. & $\begin{array}{l}\text { Incident } \\
\text { Particle } \\
\end{array}$ & $\begin{array}{c}\text { Incident } \\
\text { Particle } \\
\text { Energy (Gev) }\end{array}$ & Target & Measured quantity & $\begin{array}{l}\text { Estimate of } \\
\text { Experimental } \\
\text { Error }(\%) \\
\end{array}$ & Detector & $\begin{array}{c}\text { Installation and } \\
\text { Accelerator }\end{array}$ & References & Comments \\
\hline 642 & $\pi^{-}$. & 0.125 & $\mathrm{~Pb}$ & $\begin{array}{l}\sigma_{\pi^{-}}, \pi^{-1}\left(E_{\pi^{-}}, \psi_{\pi^{-1}}\right) \\
\text { for } E_{\pi^{-1}}<85 \mathrm{Mev}\end{array}$ & 20 & $\begin{array}{l}\text { Cloud } \\
\text { Chamber }\end{array}$ & $\begin{array}{l}\text { Columbia, } \\
\text { Cyclotron }\end{array}$ & $\begin{array}{l}\text { J.O. Kessler and L.M. } \\
\text { Lederman, Phys. Rev. } \\
94,689 \text { (1954) }\end{array}$ & \\
\hline . & & . & r. & $\begin{array}{l}\sigma_{\pi^{-} T} ; \quad \sigma_{\pi^{-}, 1 \pi^{\circ}} ; \\
\sigma_{\pi^{-} D}+\sigma_{\pi^{-}, 1 \pi^{\circ}}\end{array}$ & & . & & & \\
\hline 643 & $\pi^{-}$ & 0.140 & $\mathrm{~Pb}$ & $\sigma_{\pi T}, \sigma_{\pi-X}$ & $5-10$ & - & - & $\begin{array}{l}\text { A.E. Ignatenko et al., } \\
\text { Soviet Phys. -JETP } \frac{1}{4} \\
35 \text { (1957) }\end{array}$ & \\
\hline 644 & $\pi^{-}$ & 0.216 & $\mathrm{~Pb}$ & $\sigma_{\pi=T}, \dot{\sigma}_{\pi-x}$ & $5-10$ & - & - & $\begin{array}{l}\text { A.E. Ignatenio et al., } \\
\text { Soviet Phys. } \sqrt{\mathrm{E}} \frac{\mathrm{TP}}{4} \\
35(1957)\end{array}$ & \\
\hline 645 & $\pi^{-}$ & 0.230 to 0.250 & $\mathrm{~Pb}$ & $\sigma_{\pi-X}, \sigma_{\pi^{-}, \pi^{-1}}\left(\psi_{\pi^{-1}}\right)$ & 10 & $\begin{array}{l}\text { Cloud } \\
\text { Chamber }\end{array}$ & $\begin{array}{l}\text { Institute for } \\
\text { Nuclear Problems, } \\
\text { Synchrocyclotron }\end{array}$ & $\begin{array}{l}\text { V.P. Dzhelepov et al., } \\
\text { Soviet Phys. JETT } \\
864 \text { (1957) }\end{array}$ & \\
\hline 646 & $\pi^{-}$ & 0.256 & $\mathrm{~Pb}$ & $\sigma_{\pi T T}, \sigma_{\pi x}$ & $5-10$ & - & - & $\begin{array}{l}\text { A.E. Ignatenko et al., } \\
\text { Soviet Phys. -JETP } \frac{14}{45 \text { (1957) }}\end{array}$ & . \\
\hline 647 & $\pi^{-}$ & 0.290 & $\mathrm{~Pb}$ & $\sigma_{\pi-T}, \sigma_{\pi X X}$ & $5-10$ & - & - & $\begin{array}{l}\text { A.E. Ignatenko et al., } \\
\text { Soviet Phys. JETP } \\
\text { (1957) }\end{array}$ & \\
\hline 648 & $\pi^{-}$ & 0.350 & $\mathrm{~Pb}$ & $\sigma_{\pi-T}, \sigma_{\pi X X}$ & $5-10$ & - & - & $\begin{array}{l}\text { A.E. Ignatenko et } \frac{a l}{4} \text {, } \\
\text { Soviet Phys. JETP } \\
35 \text { (1957) }\end{array}$ & \\
\hline 649 & $\pi^{-}$ & 0.970 & $\mathrm{~Pb}$ & $\sigma_{\pi} \mathrm{X}$ & 5 & $\begin{array}{l}\text { Toluene- } \\
\text { Terphenyl } \\
\text { Scintillator }\end{array}$ & $\begin{array}{l}\text { Brookhaven, } \\
\text { Cosmotron }\end{array}$ & $\begin{array}{l}\text { J.W. Cronin, R. Cool, and } \\
\text { A. Absshian, Phys. Rev. } \\
\text { 107, } 1121 \text { (1957) }\end{array}$ & \\
\hline
\end{tabular}




\begin{tabular}{|c|c|c|c|c|c|c|c|c|c|}
\hline No. & $\begin{array}{l}\text { Incident } \\
\text { Particle }\end{array}$ & $\begin{array}{l}\text { Incident } \\
\text { Particle } \\
\text { Energy (Gev) }\end{array}$ & Target & $\begin{array}{c}\text { Es } \\
\text { Measured Quantity } \quad E\end{array}$ & $\begin{array}{l}\text { Estimate of } \\
\text { Experimental } \\
\text { Error }(\phi)\end{array}$ & Detector & $\begin{array}{l}\text { Installation and } \\
\text { Accelerator }\end{array}$ & References & Comments \\
\hline 650 & $\pi^{-}$ & 1.5 & $\mathrm{~Pb}$ & $\sigma_{\pi}-x$ & 5 & $\begin{array}{l}\text { Cloud } \\
\text { Chamber }\end{array}$ & $\begin{array}{l}\text { Brookhaven, } \\
\text { Cosmotron }\end{array}$ & $\begin{array}{l}\text { ‥ Bowen et al., } \\
\text { Fuovo cimento } 9 \\
908 \text { (1958) }\end{array}$ & \\
\hline 651 & $\vec{\pi}$ & $0.5-5.0$ & $\mathrm{~Pb}$ & $\begin{array}{l}\text { Fraction of primary } \\
\text { energy removed by } \pi^{\circ}\end{array}$ & 25 & $\begin{array}{l}\text { Cloud } \\
\text { Chamber }\end{array}$ & $\begin{array}{l}\text { Padova, Lab della } \\
\text { Marmolada }\end{array}$ & $\begin{array}{l}\text { M. Cresti et al } \\
\text { cimento } 4, \frac{1}{74} \frac{\text { Nuovo }}{7}(1956)\end{array}$ & \\
\hline 652 & $\pi^{-}$ & $6.0 \mathrm{Gev} / \mathrm{c}$ & $\mathrm{Pb}$ & $\sigma_{\pi-x}$ & 3 & $\begin{array}{l}\text { Freon } \\
\text { Bubble } \\
\text { Chamber }\end{array}$ & $\begin{array}{l}\text { Un. of Fribourg, } \\
\text { Switz.; CERN } \\
\text { Synchrotron }\end{array}$ & $\begin{array}{l}\text { R.N. Peacock et al., } \\
\text { Muovo cimento } 22,1290 \\
\text { (1961) }\end{array}$ & \\
\hline 653 & $\pi^{-}$ & $11.3 \mathrm{G} ə \mathrm{v} / \mathrm{c}$ & $\mathrm{Pb}$ & $\sigma_{\pi^{*} \mathrm{X}}$ & 3 & $\begin{array}{l}\text { Freon } \\
\text { Bubble } \\
\text { Chamber }\end{array}$ & $\begin{array}{l}\text { Un. of Fribourg, } \\
\text { Switz.; CERN } \\
\text { Synchrotron }\end{array}$ & $\begin{array}{l}\text { R.N. Peacosk et al., } \\
\text { Niovo cimento 22, } 1290 \\
\text { (1961) }\end{array}$ & \\
\hline 654 & $\pi^{-}$ & $16.0 \mathrm{Gev} / \mathrm{c}$ & $\mathrm{Pb}$ & $\sigma_{\pi-X}$ & 3 & $\begin{array}{l}\text { Freon } \\
\text { Bubble } \\
\text { Chamber }\end{array}$ & $\begin{array}{l}\text { Un. of Fribourg, } \\
\text { Switz.; CERN } \\
\text { Synchrotron }\end{array}$ & $\begin{array}{l}\text { R.N. Peacook et al:, } \\
\text { Nuovo cimento 22, } 1290 \\
\text { (1961) }\end{array}$ & \\
\hline 655 & $\pi^{-}$ & $17.6 \mathrm{Gev} / \mathrm{c}$ & $\mathrm{Pb}$ & $\sigma_{\pi-x}$ & 3 & $\begin{array}{l}\text { Freon } \\
\text { Bubble } \\
\text { Chamber }\end{array}$ & $\begin{array}{l}\text { Un. of Fribourg, } \\
\text { Switz.; CERN } \\
\text { Synchrotron }\end{array}$ & $\begin{array}{l}\text { R.N. Peacoek et al.; } \\
\text { Nuovo cimento } 22 \text { i29o } \\
\text { (1961) }\end{array}$ & \\
\hline 656 & $\pi^{-}$ & 0.160 & $\begin{array}{l}\text { Heavy Bmul- } \\
\text { sion Nuclei }\end{array}$ & $\begin{array}{l}\text { Spectrum of } \pi^{-} \\
\text {scattered from } 90^{\circ} \\
180^{\circ}\end{array}$ & - & - & $\begin{array}{l}\text { Institute for } \\
\text { Nuclear Problems, } \\
\text { Synchrocyclotron }\end{array}$ & $\begin{array}{l}\text { L.P. Kudrin and B.A. } \\
\text { Mikolskii, Soviet Phys. } \\
\text { Doklady } 1,708 \text { (1956) }\end{array}$ & \\
\hline 657 & $\pi^{-}$ & 4.3 & $\begin{array}{l}\text { Heavy Emul- } \\
\text { sion Nuclei }\end{array}$ & $\begin{array}{l}\text { Emission of } \mathrm{Li}^{8}, \mathrm{Li}^{9} \\
\mathrm{~B}^{8} \text { fragments. Energi } \\
\text { and angular dist. of } \\
\text { above }\end{array}$ & i & - & $\begin{array}{l}\text { Yamanashi Universi.. } \\
\text { ty, Japan; Plates } \\
\text { exposed at Berke- } \\
\text { ley Bevatron }\end{array}$ & $\begin{array}{l}\text { K. Imaeda, M. Kazuno, and N. } \\
\text { Ito, J. Phys. Soc. Japan } \\
15,1753 \text { (1960) }\end{array}$ & \\
\hline
\end{tabular}




\begin{tabular}{|c|c|c|c|c|c|c|c|c|c|}
\hline No. & $\begin{array}{l}\text { In:ident } \\
\text { Particle }\end{array}$ & $\begin{array}{l}\text { Incident } \\
\text { Particle } \\
\text { Energy (Gev) }\end{array}$ & Target & $\begin{array}{rr}\text { Esti } \\
\text { Measured Quantity } \\
\text { Err }\end{array}$ & $\begin{array}{l}\text { timate of } \\
\text { sperimental } \\
\text { irror (\$) }\end{array}$ & Detector & $\begin{array}{l}\text { Installation and } \\
\text { Accelerator }\end{array}$ & References & Comments \\
\hline 658 & $\pi^{-}$ & 4.3 & $\begin{array}{l}\text { Heavy Emul- } \\
\text { sion Nuclei }\end{array}$ & $\begin{array}{l}\text { Angular and energy } \\
\text { dist. of } L i^{8} \text { fragments }\end{array}$ & - & - & $\begin{array}{l}\text { Osaka City Univer- } \\
\text { sity; plates Ex- } \\
\text { posed at Berkeley } \\
\text { Bevatron }\end{array}$ & $\begin{array}{l}\text { M.T. Ohta, J. Phys. Soc. } \\
\text { Japen 15, } 2187(1960)\end{array}$ & . \\
\hline 659 & $\pi^{-}$ & $17 \mathrm{Gev} / \mathrm{c}$ & $\begin{array}{l}\text { Heavy Emul- } \\
\text { sion Nuclei }\end{array}$ & $\begin{array}{l}\text { Angular and energy } \\
\text { dist. of } \mathrm{Li}^{\theta} \text { and } \mathrm{L}, \mathrm{i}^{\Theta} \\
\text { fragments }\end{array}$ & - & - & $\begin{array}{l}\text { Strasbourg-Cronin- } \\
\text { bourg, France; } \\
\text { CERN } \\
\text { Synchrocyclotron }\end{array}$ & $\begin{array}{l}\text { G. Baumann, H. Braun, and } \\
\text { P. Cüer, J. Phys. Radium 23, } \\
335 \text { (1962) }\end{array}$ & \\
\hline 660 & $\pi^{-}$ & 0.560 & $\begin{array}{l}\text { Emulsion } \\
\text { Nuclei }\end{array}$ & $\begin{array}{l}\text { Heavy particle dist,, } \\
\text { proton energy spectrum, } \\
\text { star dist., pion ang, } \\
\text { distribution }\end{array}$ & $5-40$ & - & Upsala & $\begin{array}{l}\text { A. Frisk et al., Arkiv } \\
\text { Fysik } 19,69 \text { (1961) }\end{array}$ & . \\
\hline 661 & $\pi^{-}$ & 0.750 & $\begin{array}{l}\text { Emulsicn } \\
\text { Nuclei }\end{array}$ & Mean free path & 10 & - & $\begin{array}{l}\text { Durham; Plates } \\
\text { Exposed at Brook- } \\
\text { haven Cosmotron }\end{array}$ & $\begin{array}{l}\text { J.E. Alleñ at al., } \\
\text { Phil. Mag. } \underline{6} \text {, } \frac{\text { al }}{833} \text { (1961) }\end{array}$ & \\
\hline 662 & $\pi^{-}$ & 0.750 & $\begin{array}{l}\text { Emulision } \\
\text { Nuclei }\end{array}$ & $\begin{array}{l}\text { Angular dist. of } \pi^{-} \\
\text {fragment analysis }\end{array}$ & - & - & $\begin{array}{l}\text { Brookhaven, } \\
\text { Cosmotron }\end{array}$ & $\begin{array}{l}\text { M. Blau and O.R. Oliver, } \\
\text { Phys. Rev. 102, } 489 \text { (1956) }\end{array}$ & \\
\hline 663 & $\pi^{-}$ & 1.5 & $\begin{array}{l}\text { Enulsion } \\
\text { Nuclei }\end{array}$ & $\begin{array}{l}\text { Charged pion spectrum, } \\
\text { pions per star, angular } \\
\text { dist. of pions }\end{array}$ & Lar - & - & $\begin{array}{l}\text { Brookhaven, } \\
\text { Cosmotron }\end{array}$ & $\begin{array}{l}\text { J.E. Crew and R.D. HiIl, } \\
\text { Phys. Rev. I10 } 177 \text { (1958) }\end{array}$ & \\
\hline 664 & $\pi^{-}$ & 1.5 & $\begin{array}{l}\text { Emulsion } \\
\text { Nuclei }\end{array}$ & $\begin{array}{l}\text { Stars vs. Prongs; } \\
\text { ang. dist. of prongs }\end{array}$ & - & - & $\begin{array}{l}\text { Brookhaven, } \\
\text { Cosmotron }\end{array}$ & $\begin{array}{l}\text { R.D. H111 et al., Phys. Rev. } \\
\text { 101, } 1127 \text { (1956) }\end{array}$ & $\cdot$ \\
\hline 665 & $\pi^{-}$ & 2 & $\begin{array}{l}\text { Thulsion } \\
\text { Nuclei }\end{array}$ & $\begin{array}{l}\text { Percent of stars with } \\
\text { charged pions vs } E \pi \text {, } \\
\text { energy spectrum and ang. } \\
\text { dist. if charged } \\
\text { pions }\end{array}$ & 10 & - & $\begin{array}{l}\text { University of } \\
\text { Stockholm Ex- } \\
\text { posure Made at } \\
\text { Berkeley Bevatron }\end{array}$ & $\begin{array}{l}\text { B. Ronne and } 0 . \text { Daniels son, } \\
\text { Arkiv Fysik 22, } 175 \\
(1962)\end{array}$ & \\
\hline
\end{tabular}




\begin{tabular}{|c|c|c|c|c|c|c|c|c|c|}
\hline No. & $\begin{array}{l}\text { Incident } \\
\text { Particle }\end{array}$ & $\begin{array}{c}\text { Incident } \\
\text { Parti:le } \\
\text { Energy (Gev) }\end{array}$ & Target & Measured Quantity & $\begin{array}{l}\text { Sitimate of } \\
\text { Experimental } \\
\text { Error (\%) }\end{array}$ & Detector & $\begin{array}{l}\text { Installation and } \\
\text { Accelerator }\end{array}$ & References & Corments \\
\hline 666 & $\pi^{-}$ & 4.2 & $\begin{array}{l}\text { Emulsion } \\
\text { Nuclei }\end{array}$ & $\begin{array}{l}\text { Mean pion and } \\
\text { proton multipli- } \\
\text { city. Angular } \\
\text { distribution and } \\
\text { energy spectra for } \\
\pi^{\prime} \text { s and protons }\end{array}$ & - & - & $\begin{array}{l}\text { Weizmann Insti- } \\
\text { tute; Plates } \\
\text { Supplied by } \\
\text { Rochester }\end{array}$ & $\begin{array}{l}\text { P. Abrahamson, J. Ben- } \\
\text { Arieh, and 3. Yekutielli, } \\
\text { Nuovo cimento 12, } 27 \\
\text { ( }=959)\end{array}$ & . \\
\hline 667 & $\pi^{-}$ & 4.5 & $\begin{array}{l}\text { Enulsion } \\
\text { Nuclei }\end{array}$ & $\begin{array}{l}\text { Transverse momentum } \\
\text { of secondary pions. } \\
\text { Shower particle mul- } \\
\text { plicity and angular } \\
\text { distribution }\end{array}$ & $5-10$ & - & $\begin{array}{l}\text { Bristol; Exposure } \\
\text { Made at Berkeley } \\
\text { Bevatron }\end{array}$ & $\begin{array}{l}\text { H.H. Aly, J.G.M. Duthie, } \\
\text { and C.M. Fisher, Phil. Mag. } \\
\text { 4, } 993 \text { (1959) }\end{array}$ & \\
\hline 668 & $\pi^{-}$ & 4.5 & $\begin{array}{l}\text { Enulsion } \\
\text { Nuclei }\end{array}$ & $\begin{array}{l}\text { Angular distribution } \\
\text { of secondary } \\
\text { charged } \pi \text { mesons }\end{array}$ & - & - & $\begin{array}{l}\text { 3risto1; Exposure } \\
\text { Made at. Berkeley } \\
\text { 3evatron }\end{array}$ & $\begin{array}{l}\text { H.H. Aly and C.M. } \\
\text { F:sher, Nuovo cimento } \\
\underline{1} \text {, } 98(1960)\end{array}$ & \\
\hline 669 & $\pi^{-}$ & 4.5 & $\begin{array}{l}\text { Emulsion } \\
\text { Nuclei }\end{array}$ & $\begin{array}{l}\text { Shower tracks } \\
\text { per star }\end{array}$ & - & - & $\begin{array}{l}\text { Rajshahi University; } \\
\text { Exposure Made at } \\
\text { Dacca University }\end{array}$ & $\begin{array}{l}\text { A. Husain and M.H. Siddique, } \\
\text { Nuovo =imento II } \\
438 \text { (1959) }\end{array}$ & \\
\hline 670 & $\pi^{-}$ & $7.3 \mathrm{Gev} / \mathrm{c}$ & $\begin{array}{l}\text { Emulsion } \\
\text { Nuclei }\end{array}$ & $\begin{array}{l}\text { Average star char- } \\
\text { acteristics with pion, } \\
\text { proton, deuteron or } \\
\text { triton secondaries and } \\
\text { average zinematic prop- } \\
\text { erties of latter }\end{array}$ & p- & - & $\begin{array}{l}\text { Joint Institute } \\
\text { for Nuclear } \\
\text { Research, } \\
\text { Synchrotron }\end{array}$ & $\begin{array}{l}\text { E. Balea et al., Nuovo } \\
\text { cimento } 25 \text {, } \frac{1}{21} 4 \text { (1962) }\end{array}$ & \\
\hline 671 & $\pi^{-}$ & $17 \mathrm{Gev} / \mathrm{c}$ & $\begin{array}{l}\text { Emulsion } \\
\text { Nuclei }\end{array}$ & $\begin{array}{l}\text { Hyper fragment } \\
\text { production }\end{array}$ & - & - & $\begin{array}{l}\text { Ixposed at } \\
\text { CERN Synchrotron }\end{array}$ & $\begin{array}{l}\text { G. Baumann, H. Braun, } \\
\text { and P. Cüer, Compt. rend. } \\
254,3839 \text { (1962) }\end{array}$ & \\
\hline 672 & $\pi^{+}$or $\pi^{-}$ & 0.048 & c & $\sigma_{\pi, \pi^{\prime}}$ & 30 & Cloud Chamber & $\begin{array}{l}\text { Cornell, } \\
\text { Symchrotron }\end{array}$ & $\begin{array}{l}\text { A.M. Shapiro, Phys. Rev. } \\
84,1063 \text { (1751) }\end{array}$ & \\
\hline
\end{tabular}




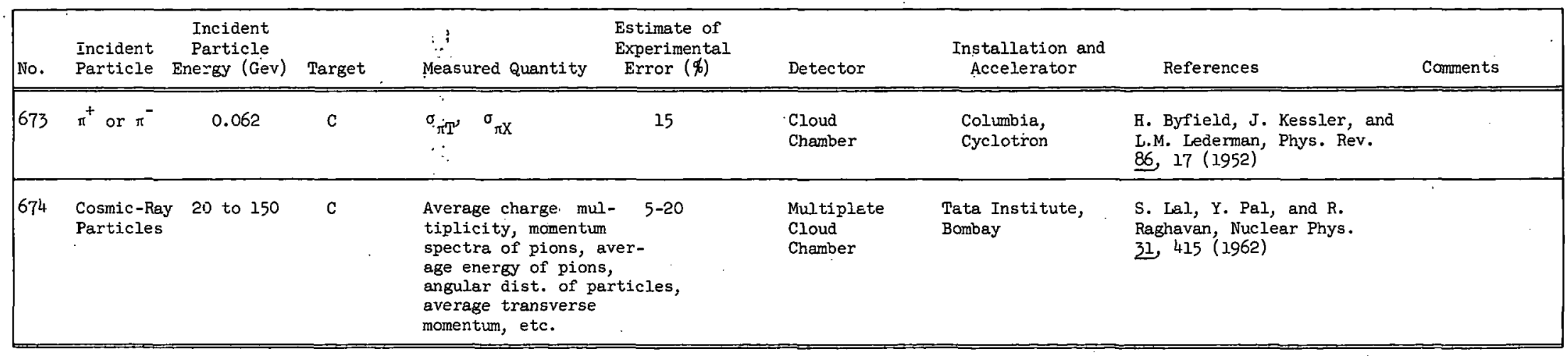




\section{BIBLIOGRAPHY}

Abbatista, N., et al., Nuovo Cimento 23, 1 (1962).

(6II)

Abrahamson, P., J. Ben Areih, and G. Yekutielli, Nuovo Cimento 12, 27 (1959). (666)

Albouy, G., M. Gusakow, and N. Poffe, Rutherford Jubilee International Conference, Manchester, 1961, Paper C 1/26, p. 191, Heywood, London. (373)

Alekseeva, K. I., and N. L. Grigorov, Soviet Phys. "Doklady" (English Transl.)

2) 317 (1957).

(117)

Alekseeva, K. I., and N. L. Grigorov, Soviet Phys. JETP (English Transl.) 5, 339 (1957).

(114)

Alekseeva, K. I., and N. L. Grigorov, Soviet Phys. JETP (English Transl.) 8, 416 (1959).

Allen, J. E., et al., Phil. Mag. 6, 833 (1961). (661)

Aly, H. H., J. G. M. Duthie, and C. M. Fisher, Phil. Mag. 4, 993 (1959). (667)

Aly, H. H., and C. M. Fisher, Nuovo Cimento 17, 98 (1960). $(482,668)$

Arifkhanov, U. R., et al., Soviet Phys. JETP (English Transl.) 11, 806 (1960).

$(438,450)$

Ashmore, A., et al., Proc. Phys. Soc. (London) 70A, 745 (1957).

(495, 509, 518, 525, 537, 54.7, 557)

Assovskaya, A. S., and N. S. Ivanova, Soviet Phys. JETP (English Transl.) 12. 1051 (1961). 
Atkinson, J.H., et al., Phys. Rev. Letters 2, 168 (1959). $(515,544,564)$

Austin, S., et al., Rutherford Jubilee Internátional Conference, Manchester, 1961, Paper C 1/1, p. 139, Heywood, London.

$(55,507)$

Austin, S. M., et al., Proc. Phys. Soc. (London) 80, 383 (1962). $(56,506)$

Azhgirei, I. S., et al., Soviet Phys. JETP (English Transl.) 7, 939 (1958). (93)

Azhgirei, L. S., et al., Nucl. Phys. 13, 258 (1959). $(27,85,275,430)$

Baiukov, Iu. D., M. S. Kozodaev, and A. A. Tiapkin, Soviet Phys. JETP (English Transl.) 5, 552 (1957). $(78,94)$

Baker, E., G. Friedlander, and J. Hudis, Phys. Rev. 112,1319 (1958). (101, 102, 104, 108, 211, 212, 21.4, 215, 281, 285, 286, 322-324, 387-389)

Baker, E. W., and S. Katcoff, Phys. Rev. 123, 641 (1961). $(463,466,468)$

Baker, E. W., S. Katcoff, and C. P. Baker, Phys. Rev. 117, 1352 (1960). $(462,465,467)$

Baker, W. F., èt al., Phys. Rev. Letters I, 101 (1961). (35-38, 220-222, 224)

Balea, i., et al., Nuovo Cimento 25, 214 (1962). $(491,670)$

Barrett, P. H., Phys. Rev. 114, 1374 (1959). (531, 543, 563)

Baumann, G., H. Braun, and P. Cuer, Compt. Rend. 254, 1966 (1962). (473)

Baumann, G., H. Braun, and P. Cuer, Compt. Rend. 254, 2549 (1962). $(474)$ 
Baumann, G., H. Braun, and P. Cuer, Compt. Rend. 254, 3839 (1962). (671)

Baumann, G., H. Braun, and P. Cuer, J. Phys. Radium 23, 335 (1962). (659)

Bayatyan, G. L., et al., Soviet Phys. JETP (English Transl.) 9, 483 (1959). (487)

Belyaev, B. N., A. V. Kalyamin, and A. N. Murin, Soviet Phys. "Doklady" (English Transl.) 6, 784 (1962). (407)

Benioff, P. A., Phys. Rev. 119, 316 (1960). (34, 112, 135, 153, 171, 176, 218)

Bercovitch, M., et al., Phys. Rev. 119, 412 (1960). (339; 367, 400, 428)

Bernardini, G., E. T. Booth, and S. J. Lindenbaum, Phys. Rev. 도, 826 " (1952). $(454,572)$

Blan, M., and 0. R. Oliver, Phys. Rev. 102, 489 (1956). (662)

Bogachev, N. P., et al., J. Nucl. Energy 9, 178 (1959). (488)

Bogachev, N. P., et al., Soviet Phys. JEMP (English Transl.) 11] 317 (1960). (469)

Booth, N. E., et al., Proc. Phys. Soc. (London) 70A, 209 (1957). $(97,209,280,342)$

Booth, N. E., G. W. Hutchinson, and B. Ledley, Proc. Phys. Soc. (London) 71, 293 (1958). $(497,498,512,521,528,540,552,560)$

Bouissieres, G., et all., Compt. Renk. 251, 2155 (1960). (414)

Bowen, P. H., et al., Nucl. Phys. 30, 475 (1962). $(1,7,17,52,187,263,396,424)$ 
Bowen, T., et al., Nuovo Cimento 9, 908 (1958).

$(105,252,403,615,624,650)$

Bricman; C., et al., Nuovo Cimento 20, 1017 (1961).

(492)

Brun, C., M. Lefort, and X. Tarrago, J. Phys. Radium 23, 167 (1962).

$(53,181,188,246,311,336,370,412)$

Byfield, H., J. Kessler, and L. M. Lederman, Phys. Rev. 86, 17 (1952). (673)

Caretto, A. A., and G. Friedlander, Phys. Rev. 110, 1169 (1958).

(352, 354-356)

Caris, J., et al., Phys. Rev. 126, 295 (1962).

$(581,584,586,588)$

Chen, F'. F.; C. P. Leavitt, and A. M. Shapiro, Phys. Rev. 99, 857 (1955). $(502,513,529,541,550,561)$

Cladis, J. B., W. N. Hess, and B. J. Moyer, Phys. Rev. 87, 425 (1952). $(2,70)$

Cocconi, G., et al., Phys. Rev. 126, 277 (1962).

(118, 119, 122)

Coor, T., et al., Phys. Rev. 98,1369 (1955).

$(503,514,530,542, .551,562,565,571)$

Corte, D. M., T. F. Fazzini, and A. M. Sona, Nuovo Cimento 2, 1345 (1955). $(580,606)$

Crandall, W. E., and G. P. Millburn, J. Appl. Phys. 29. 698 (1958). (197, 267, 325, 417, 425, 522, 533, 545, 566, 567, 569)

Cresti, M., et al., Nuovo Cimento 4, 747 (1956). (591, 651)

Crew, J. E., and R. D. Hill, Phys. Rev. 110, 177 (1958). (663)

Cronin, J. W., R. Cool, and A. Abashian, Phys. Rev. 107, 1121 (1957). $(605,609,610,613,614,622,623,631,641,649)$ 
Crozon, M., and J. Tocqueville, Compt. Rend. 254, 1963 (1962). $(604,612,630)$

Cuming, J. B., et al., Phys. Rev. 127. 950 (1962). (223)

Cumming, J. B., et al., Phys. Rev. 128, 2392 (1962). $(116,219)$

Cumming, J. B., G. Friedlander, and S. Katcoff, Phys. Rev. 125, 2078 (1958).

(123)

Cumming, J. B., G. Friedlander, and C. E. Swartz, Phys. Rev. 111, 1386 (1962).

$(103,109,213,216)$

Darovskikh, V. F., N. P. Kocherov, and N. A. Perfilov, Soviet. Phys. JETP

(English Transl.) 10, 921 (1960).

(409)

De Juren, J., and N. Knable, Phys. Rev. 77, 606 (1950). $(494,499,505,516,517,523,532,535,546,555,568)$

Dzhelepov, V. P., et al., Soviet Phys. JETP (English Transl.) 4, 864 (1957). $(608,645)$

Dzhelepov, V. P., K. O. Oganesian, and V. B. Fliagin, Soviet Phys. JETP

(English Transl.) 5, 560 (1957).

$(496,500,510,519,526,538,548,553,558)$

E -- None

Fiorini, E., and S. Ratti, Nuovo Cimento 14, 901'(1959).

$(67,142)$

Fitch, V., S. Meyer, and P. Piroué, Phys. Rev. 126, 1849 (1962).

$(39,40,225,226)$

Fox, R., and N. F. Ramsey, Phys. Rev. 125, 1609 (1962).

(294, 337, 358, 397) 
Friedländẹ, E., M. Marcu, and M. Spirchez, Rev. Acad. Rep. Populaire Roumaine Phys. 6; 457 (1961).

(449)

Friedlander, G., and L. Yoffe, Phys. Rev. 117, 578 (1959). (404, 436)

Frisk, A., et al., Arkiv Fysik 19, 69 (1961). (660)

Gajewșki, w., et al., Nucl. Phys. 37, 226 (1962). (470)

Garron, J.P., et al., Nucl. Phys. 37, 126 (1962). $(8,9,42,43,58)$

Génin, J., et al.,.J. Phys. Radium 22, 615 (1961). (377)

Goebel, K., et al., Nucl. Phys. 24, 28 (1961). $(82,204)$

Go ishi, W., and W. F. Tibby, Phys. Rev. 104, 1717 (1956). (636)

Goloskie, R., and K. Strauch, Nucl: Phys. 29, 474 (1962). $(46,47,49,50,180,182,184,185,256,258,260,262,315,316$, $318,319,391,392,394,395)$

Gooding, T. J., and H. G. Puch, Nucl. Phys. 18, 46 (1960). (57)

Gordon, G. D., et a1., Phys. Rev. 108, 1315 (1957). (616)

Gorichev, P. A., O. V. Lozhkin, and N. A. Perfilov, Soviet Phys. JETP (English Transl.) 41, 27 (1962). (471)

Gotchell, E. K., Phys. Rev. 105, 713 (1957). (12) 
Gradsztajn, E., J. Phys. Radium 21, 761 (1960). (138)

Grover, J., Phys. Rev. 126, 1540 (1962).

(362)

Gusakow, M., Ann. Phys. (Paris) 7, 67 (1962).

$(154,172,244,306,343,371)$

Gusakow, M., et al., Compt. Rend. 251, 70 (1960). (374)

Gusakow, M., et al., J. Phys. Radium 22, 636 (1961).

(155, 173, 245, 344, 372)

Hadley, J., and H. York, Phys. Rev. 80, 345 (1950).

$(504,534,554)$

Hill, R. D., et al., Phys. Rev. 101, 1127 (1956). (664)

Honda, M., and D. Lal, Phys. Rev. 118, 1618 (1960). $(61,66,74,95,250,251)$

Horwitz, I., and J. J. Murray, Phys. Rev. 117, 1361 (1960). (106, 111, 113)

Hunter, E. T., and J. M. Miller, Phys. Rev. 115, 1053 (1959). (408)

Ilusain, A., and M. H. Siddique, Nuovo Cimento 11, 438 . (1959). (669)

Ignatenko, A. E., et al., Soviet Phys. JETP (English Transl.) 4 , 351 (1957). $(599-603,617-629,643,644,646-648)$

Imaeda, K., M. Kazuno, and N. Ito, J. Phys. Soc. Japan 15, 1753 (1960). (657)

Ivanova, N. S., V. I. Ostroumov, and Yu. V. Pavlov, Soviet Phys. JETP

(English Transl.) 10, 1037 (1960). $(593,594)$ 
Jain, P. L., et al., Nruovo Cimento 21, 859 (1961).

(493)

Johansson, A., U. Svanberg, and O. Sundberg, Arkiv Fysik 19, 527 (1961).

$(10,18,59,192,241,248,327,380)$

Kaufman, S., Phys. Rev. 126, 1189 (1962).

(305)

Kavanagh, T. M., and R. E. Bell, Can. J. Phys. 39, 1172 (1961).

(369)

Kessler, J. O., and L. M. Lederman, Phys. Rev. 24, 689 (1954). $(607,642)$

Kiselev, V. S., and V.: B. Fliagin, Soviet Phys. JETP (English Transl.)

5,786 (1957).

(3I)

Kocharian, N. M., et al., Soviet Phys. "Doklady" (English Transl.) 1, 209 (1956).

$(282,284,290,291)$

Kozodaev, M. S., et al., Soviet Phys. JETP (English Transl.) 11, 300 (1960).

$(574,598)$

Kudrin, L. P., and B. A. Nilolskii, Soviet Phys. "Doklady" (English Transl.) 1, 708 (1956).

(656)

Kuznetsova, M. Ya., V. N. Pokrovskii, and V. N. Rybakov, Soviet Phys. JETP (English Transl.) 42, 1451 (1962).

(199)

Laberrigue-Frolova, J. V., M. P. Balandin, and S. Z. Otvinovskii, Soviet Phys. JETP (English Transl.) 10, 452 (1960).

(578)

Labrukhin, A. K., and A. A. Pozdnyakov, Soviet J. At. Energy (English Trans1.) I, 862 (1960).

(357) 
Ladenbauer, I. M., and L. Winsberg, Phys. Rev. 119, 1368 (1960). (331)

Lal, S., Y. Pal, and R. Raghavan, Nucl. Phys. 31, 415 (1962). (674)

Lavrukhina, A. K. Soviet Phys. "Doklady" (English Transl.) 4, 1317 (1959). $(230,277)$

Lavrukhina, A. K., et al., Soviet J. At. Energy (English Transl.) 3, 1087 (1957).

$$
(261,264,268,272,276,3) \cdot 6-350,376,381,383,385,386,411 \text {, }
$$$$
416,418,419,420)
$$

Lavrukhina, A. K., G. M. Kolesov, and T. Syao-en, Bull. Acad. Sci. USSR

Phys. Ser. (English Transl.) 24, 1117 (1960). (353)

Law, M. E., G. W. Hutchinson, and D. H. White, Nucl. Phys. 9, 600 (1959). $(4,98,151)$

Lefort, M., Compt. Rend. 253, 2221 (1961). (423)

Lefort, M., G. Simonoff, and X. Tarrago, J. Phys. Radium 21, 338 (1960). (413)

Likhachev, M. F., et al., Soviet Phys. JETP (English Transl.) 14, 29 (1962). $(582,585,587,589,592)$

Lillethun, E., Phys. Rev. 125, 665 (1962). $(24,76,200,271,338,341,384,398,426)$

Longo, M. J., and B. J. Moyer, Phys. Rev. 125, 701 (1962). $(33,107,217,387)$

Lozhkin, O. V., Soviet Phys. JETP (English Transl.) 6, 273 (1958) . $(439-443 ; 452,453,455,457,458)$

Lozhkin, O. V., et al., Soviet Phys. JETP (English Transl.) 11, 1001 (1960). (444, 460)

Lozhkin, O. V., and N. A. Perfilov, Soviet Phys. JETP (English Transl.)

4, 790 (1957). $(475-478)$ 
Lukin, Yu. T., Zh. S. Takibaev, and.E. V. Shalagina, Soviet Phys. JETP (English Transl.) 11, 776 (1960).

Malysheva, T. V., and I. P. Alimarin, Soviet Phys. JETP (English Transl.) 8, 772 (1959). (410)

Markowitz, S. S.,.F. S. Rowland, and G. Friedlander, Phys. Rev. 112, 1295 (1958).

(127, 134, 163, 169, 170, 249, 253-255, 269, 270, 283, 288, 289, 296, $297,312-314,359-361)$

McEwen, J. G., W. M. Gibson, and P. J. Duke, Phil. Mag. 2, 231 (1957). (480)

Mertz, E., and A. Caretto, Jr., Phys. Rev. 126, 1173 (1962). (265)

Meshcheriakov, M. G., et al., Soviet Phys. JEIP (English Transl.) 4, 79 (1957). $(28,86)$

Meshkovskii, A. G., et al., Soviet Phys. JEPP (Englïsh Transl.) 4, 842 (1957).

$(13,29 ; 87,206,278,321,402)$

Meshkovskii, A. G., et al., Soviet Phys. JETP (English Transl.) 5, 1085 (1957).

$(3,88)$

Meshkovskii, A. G., Ia. Ia. Shalamov, and V. A. Shebanov, Soviet Phys. JETP (English Transl.) 6, 463 (1958).

$(14,30,207,279)$

Meshkovskii, A. G., Ia. Ia. Shalamov, and V. A. Shebanov, Soviet Phys. JETP (English Transl.) 7, 987 (1958).

(89) 
Meshkovskii, A. G., and Ia. Ia. Shalamov, Soviet Phys. JETP (English Transl.) 10, 697 (1960). (597)

Meyer, V., R. M. Eisberg, and F. R. Carlson, Phys. Rev. 117, 1334 (1960). $(45,179,246,335,390)$

Millburn, G. P., et al., Phys. Rev. 25, 1268 (1954). $(25,80,202,273,399,427,508,524,536,556)$

Morrison, D. L., and A. A. Caretto, Jr., Phys. Rev. 127, 1731 (1962). (229, 233, 236, 237, 243, 295, 304, 366, 368)

Morton, W. T., and B. A. Munir, Phj̇. Mag. 4, 933 (1959). (573)

Moskalev, V. I., and B. V. Gavrilovskii, Soviet Phys. "Doklady" (English Trans1.) 1, 607 (1956).

$(26,84,148,205,274,340,401,429,501,511,520,527,539$, $549,559,470)$

Neiman, F. L., et al., Phys. Rev. 108, 1331 (1957). (32)

Nethaway, D. R., and L. Winsberg, Phys. Rev. 119, 1375 (1960). (328, 329, 332, 333)

Nguyen-Long-Den., M., Compt. Rend. 253, 2919 (1961). (191)

Otha, M. T., J. Phys. Soc. Japan 15, 2187 (1960). (658)

Ostroumov, V. I.; Soviet Phys. JEIP (English Transl.) 5, 1? (1957). $(451,456,459)$

Pal, Y., and T. Rengarajan, J. Phys. Soc. Japan 17, Suppl. A-III, 387 (1962).

Pal, Y., and T. Rengarajan, Phys. Rev. 124, 1575 (1961). (120) 
Parikh, V., Nucl. Phys. 18; 628 (1960).

$(65,68,69,72,73)$

Parikh, V., Nucl. Phys. 18, 638 (1960).

$(64,196)$

Parikh, v., inucl. Phys. 18, 646 (1960). (20-2j, 140, 141, 143, 144, 158, 160-162)

Pate, B. D., and A. M. Poskanzer, Phys. Rev. 123, 647 (1961). (421, 422, 431, 432, 433,: 434, 435, 437)

Peacock, R. N., et al., Nuovo Cimento 22, 1290 (1961). $(292,326,405,632-640,652-655)$

Perfilov, N. A., et al., Soviet Phys. JETP (English Transl.) 1], 250 (1960) .

(472)

Perfilov, N. A., and Yu. I. Serebrennikov, Soviet Phys. JETP (English

Transl.) 13, 274 (1961).

(90, 131, 149)

Philberg, G., Compt. Rend. 243, 141 (1956).

$(445,461)$

Philbert, G., J. Phys. Radium 18, 656 (1957).

$(446,464)$

Philbert, G., and L. Vigneron, Compt. Rend. 247, 290 (1958). (447)

Philbert, G., and L. Vigneron, Compt. Rend. 247, 1335 (1958). (448)

Poffé, N., et al., J. Phys. Radium 2l, 343 (1960). (379)

Poffé, N., et al., J. Phys. Radium 22, 639 (1961). (375)

Poffé, N., M. Riou, and J. Teillae, Compt. Rend. 248, 3552 (1959). (378) 
Porile, N. T., Phys. Rev. 125, 1379 (1962). (298-303)

Porile, N. T., Phys. Rev. 128, 1916 (1962). $(330,334)$

Prokoshkin, Yu. D., and A. A. Tiapkin, Soviet Phys. JETP (English Transl.) 5, 12 (1957). $(54,63,71,77,81,91,189,195,198,201,203,208)$

Rajopadhye, V. Y., Phil. Mag. 5, 537 (1960). (481)

Rajopadhye, V. Y., Proc. Natl. Inst. Sci. India, Pt. A 28, 238 (1962). (483)

Riddiford, L., and A. Williams, Proc. Roy. Soc. (London, Ser. A 257 . 316 (1960).

(5)

Rcihl, C., J. Phys. Radium 22, 770 (1961). (354)

Ronne, B., and O. Danielsson, Arkiv Fysik 22, 175 (1962). (665)

Rudstam, G., E. Bruninx, and A. C. Pappas, Phys. Rev. 126, 1852 (1962). (293)

Salukvadze, R. G., and D. Neagu, Soviet Phys. JETP (English Transl.)

14, 59 (1962).

(596)

Saphir, G., Fhys. Rev. 104, 535 (1956). (590)

Segar, A. M., and R. Rubinstein, Nucl. Phys. 14, 222 (1959). $(99,210)$

Shapiro, A. M., Phys. Rev. 84, 1063 (1951). (672)

Singh, Sarjant, and J. M. Alexander, Phys. Rev. 128, 711 (1962). (110) 
Skyrme, D., Nucl. Phys. 25, 177 (1962). $(190,257,320,363-365)$

Solov'eva, L. P:, Soviet Phys. JETP (English Transl.) 4, 801 (1957). (4.79)

Strauch, K., and F. Titus, Phys. Rev. 104, 191 (1956).

(6, 15, 41, 48, 124, 136, 157, 174, 177, 183, 227, 231, 234, 259, $317,393,406)$

Symonds, J. L., J. Warren, and J. D. Young, Proc. Phys. Soc. (London) 70A, 824 (1957).

$(75,79,83,96,100,128-130,132,133,145-147,150,152$, 164-168)

Takivaev, Zh., E. Shalagina, and G. Tsadikova, Soviet Phys. "Doklady". (English Transl.) 6, 1094 (1962).

(490)

Tarrago, X., J.'Phys. Radium 23, 1, (1962). (415)

Taylor, A. E., and E. Wood, Nucl. Phys. 25, 642 (1961). $(16,51,125,137,186)$

Temmer, G. M., Phys. Rev: 83; 1087 (1951). (62)

Tenney, F. H., and J. Tinlot, Phys. Rev. 92, 974 (1953). $(569,576,577)$

Tyrén, H., P. Hillman, and Th. A. J. Maris, Nucl. Phys. I, 10 (1958). $(60,139,242)$

Tyrén, H., and Th. A. J. Maris, Nucl. Phys. 6, 82 (1958). $(11,19,44,126)$

Tyrén, H., and Th. A. J. Maris, Nucl. Phys. 6, 446 (1958). $(157,175,178,193,228,232,235,238-240)$

Ünseren, E., and E. Wiig, Phys. Rev. 122, 1875 (1961). $(307-310)$ 
Valckx, F., E. Gelsema, and N. Kruiskamp, Nuovo Cimento 23, 1005 (1962). $(579,583)$

Ware, W. R., and E. O. Wilg, Phys. Rev. 122, 1837 (1961). (351)

Winzeler, H., et al., Nuovo Cimento 17, 98 (1960). (484)

X -- None

Yule, H. P., and A. Turkevich, Phys. Rev. 118, 1591 (1960). $(159,194,266,382)$

Zhdanov, A. P., and P. I. Fedotov, Soviet Phys. JETP (English Transl.) 10, 280 (1960).

(92)

Zhdanov, G. B., et al., Soviet Phys. JETP (English Transl.) 10, 442 (1960).

(486)

Zhdanov, G. B., et al., Soviet Phys. JETP (English Transl.) 10, 433 (1960).

(485) 
THIS PAGE

\section{WAS INTENTIONALLY LEFT BLANK}




\author{
ORIVL-3455 \\ UC-34 - Physics \\ TID-4500 (20th ed., Rev.)
}

\title{
INTERINAL DISTRIBUTION
}

\author{
1. Biology Library \\ 2-3. Central Research Library \\ 4. Reactor Division Library \\ 5-6. ORNL - Y-12 Technical Library \\ Document Reference Section \\ 7-56. Laboratory Records Department \\ 57. Laboratory Records, ORNL R.C. \\ 58. R. G. Alsmiller \\ 59. J. A. Auxier \\ 60-69. H. W. Bertini \\ 70. E. P. Blizard \\ 71. Eugene Eichler \\ 72. C. B. Fulmer \\ 7.3. W. A. Gibson \\ 74. J. A. Harvey \\ 75. W. H. Jordan
}

\author{
76. C. E. Larson \\ 77. F. C. Maienschein \\ 78. J. J. Pinajian \\ 79. M. J. Skinner \\ 80. J. A. Swartout \\ 81-82. J. E. Turner \\ 83. J. W. Wachter \\ 84. A. M. Weinberg \\ 85. H. B. Willard \\ 86. C. D. Zerby \\ 87. W. Zobel \\ 88. A. Zucker \\ 89. R. A. Charpie (consultant) \\ 90. P. F. Gast (consultant) \\ 91. R. F. Taschek (consultant) \\ 92. T. J. Thumpson (consultant)
}

\section{EXTERINAL DISTRIBUTION}

93-94. Lincoln Laboratory, Massachusetts Institute of Technology, Lexington, Massachusetts (I copy each to William H. Radford and James W. Meyer)

95. Leonard S. Sheingold, Sylvania Electronic Systems, 40 Sylvan Road, Waltham, Massachusetts

96. Burton F. Miller, Thompson Ramo Wooldridge, Inc., 8433 Fallbrook Avenue, Canoga Park, California

97. C. W. Sherwin, Aerospace Corporation, Los Angeles 45, California

98. W. C. Tinus, Bell Telephone Labs, Inc., Whippany, New Jersey

99. Gilbert W. King, IBM Thomas J. Watson Research Center, Yorktown Heights, New York

100. Richard W. Porter, General Electric Company, Engineering Services, New York 22, New York

101. Maj. Julius H. Massey, Jr., Asst. Secretary, USAF Scientific Advisory Board, Washington 25, D.C.

102. Rear Adrn. Paul A. Smith, The RAND Corporation, Washington 6, D.C.

103. Albert D. Wheelon, Central Intelligence Agency, Washington, D.C.

104. Joseph Kaplan, University of California, Los Angeles 24, California

105. Brian 0'Brien, P. O. Box 117, Pomfret, Connecticut

106. James B. Edson, NASA, Code R, Washington 25, D.C.

107. E. B. Staples, Wright Patterson Air Force Base (Foreign Technology Division), Ohio

108. Miguel Awschalom, Princeton-Pennsylvania Accelerator, Princeton, New Tersey

109. Richard B. Curtis, Indiana University, Bloomington, Indiana 
110-114. Stanford University, Stanford, Califurmia ( I copy each to H. DeStaebler, Jr., K. G. Dedrick, R. F. Mozley, W. K. H. Panofsky, and J. Ballam)

115. Herman J. Schaefer, U.S. Naval School of Aviation, Pensacola, Florida

116. S. P. Shen, New York University, New York, New York

117. E. A. Cosbie, Argonne National Laboratory, Argonne, Illinois

118-120. University of California, Berkeley ( 1 copy each to B. J. Moyer R. Wallace, and W. Patterson)

121. M. Stanley Livingston, Cambridge Electron Accelerator, Cambridge, Massachusetts

122-123. NASA, Goddard Space Flight Center, Greenbelt, Maryland (1 copy each to W. N. Hess and Frank McDonald)

124-125. Bendix Systems Division, Ann Arbor, Michigan (1 copy each to 0 . L. Tiffany and K. More)

126. R. L. Childers, University of Tennessee, Knoxville, Tennessee

127. Aaron Galonsky, Midwestern Universities Research Association, Madison, Wisconsin

128-133. Brookhaven National Laboratory, Upton, Long Island, New York (I copy each to F. P. Cowan, S. J. Lindenbaum, G. Friedlander, Y. Shimamoto, Catherine Chen, and J. R. Grover)

134. H. B. Knowles; Yale University, Sloane Laboratory, New Haven Connecticut

135-136. Advance Research Corporation, Lafayette, Indiana (1 copy each to E. C. Sirlith: and W. M. Schofield)

137-138. NASA, Washington, D.C. (I copy each to J. W. Keller and Lt. Col. Joseph Conner)

139. E. O. Berdahl, Scientific Advisor. (Systems). TDXIA, Wright Patterson Air Force Base, Ohio

140-14.5. WADC, Dayton; Ohio (1 copy each to Capt. R. F. Cooper, C. A. Dempsey, Maj. J.F. Dinwiddie, T.J. McGuire; L. Pittman, and J. Speakman)

146. Harry Schulte, Bellcomm, Inc., Washington, D.C.

147. R. V. Glowczwski, McDonnell Aircraft, St. Louis, Missouri

148. R. A. Glass, Lockheed Missiles and Space Company, Dept. 52-10, Palo Alto, California

149. Glenn A. Whan, The University of New Mexico, Albuquerque, New Mexico

150. K. D. George, Reactor Requirements Office, Picatinny Arsenal, Dover, New Jersey

151. F. L. Keller, Aerospace Corporation, El Segundo, California

152. D. H. Robey, General Dynamics/Astronautics, San Diego, California

153. W. H. Langham, Los Alamos Scientific Laboratory, Los Alamos, New Mexico

154-157. NASA, Langley Field, Virginia ( 1 copy each to L. F. Vosteen, W. C. Hulton, T. Foelsche, and J. E. Duberg)

158. D. Langford; Pratt and Whitney, East Hartford, Connecticut

159-162. NASA, Manned Space Craft Center, Houston, Texas ( 1 copy each to R. H. Steelle, C. Warren, W. L. Gill, and L. N. McMillion)

163-164. NASA, Huntsville, Alabama ( 1 copy each to R. Shelton and H. E. Stern) 
165-168. North American Aviation, Downey, California (1 copy each to K. R. Pinckney, G. E. Laubach, L. Clark, and M. R. Kinsler)

169. E. R. Beever, Space and Information Systems Division, North American Aviation, Downey, California

170. J. P. T. Pearman, National Academy of Sciences, Washington, D.C.

171. D. W. Drawbaugh, Westinghouse Electric Company, Pittsburgh, Pennsylvania

172-173. USAF Aerospace Medical Center, Brooks Air Force Base, Texas ( 1 copy each to Lt. Col. Ralph G. Allen, Jr., and Col. John E. Pickering)

174. Charles Hill, Lockheed Aircraft, Marietta, Georgia

175. E. M. Finkelman, Grummon Aircraft, Bethpage, New York

176. P. Mittleman, United Nuclear Corporation, White Plains, New York

177. Ray Aronson, Technical Research Group, Syossett, New York

178. Richard Madey, Republic Aviation Corporation, Farmingdale, Long Island, New York

179-181. Boeing Aircraft, Seattle, Washington (1 copy each to D. L. Dye, M. Pearson, J. C. Noyes)

182-183. Jet Propulsion Laboratory, Pasadena, California ( I copy each to R. V. Meghreblian and D. F. Spencer)

184-185. University of California, Berkeley, California (1 copy:each to C. Sondhaus and C. Tobias)

186. L. Jackson Laslett, Chief, High-Energy Physics Branch, Division of Research, U.S. Atomic Energy Commission, Washington 25, D.C.

187-192. General Dynamics, Fort Worth, Texas ( 1 copy each to R. French, N. Schaeffer, C. F. Johnson, T. W. Deveries, T. J. Rock, and S. Dominey)

193. M. J. Berger, National Bureau of Standards, Washington, D.C.

194. John P. Neissel, General Electric Company, General Engineering Laboratory, Schenectady, New York

195. S. Krasner, Office of Naval Research, Washington, D.C.

196. E. V. Vaughan, Atomics International, Canoga Park, California

197-199. The Martin Compary, Baltimore, Maryland ( 1 copy each to $\mathrm{S}$. Russak, A. J. Beck, and E. Divita)

200-202. Northrup Space Laboratory, Los Angeles, California (1 copy each to M. C. Chapman, R. E. Fortney, and S. H. Levine)

203. William Steigelmann, Franklin Institute, Philadelphia 3, Pennsylvania

204. Capt. William A. Anders, USAF, AFSWC (SWVPF), Kirtland AFB, New Mexico

205-206. Lewis Research Center, Cleveland, Ohio ( 1 copy each to I. M. Karp and R. I. Hildebrand)

207. J. M. Miller, Chemistry Department, Columbia University, New York, New York

208. George A. Kolstad, Physics and Mathematics Programs, Division of' Research, U.S. Atomic Energy Commission, Washington, D.C.

209. Robert E. Cote, Argonne National Laboratory, 9700 South Cass Avenue, Argonne, Illinois

210. Hermann J. Donnert, Nuclear Defense Laboratory, Army Chemical Center, Maryland.

211. Stanley C. Fultz, Lawrence Radiation Laboratory, P. O. Box 808, Livermore, California 